Tobias Reeh, Gerhard Ströhlein, Axel Bader (Hg.)

\title{
Kulturlandschaft verstehen
}

\section{ZELTForum - Göttinger Schriften zu}

Landschaftsinterpretation und Tourismus - Band 5

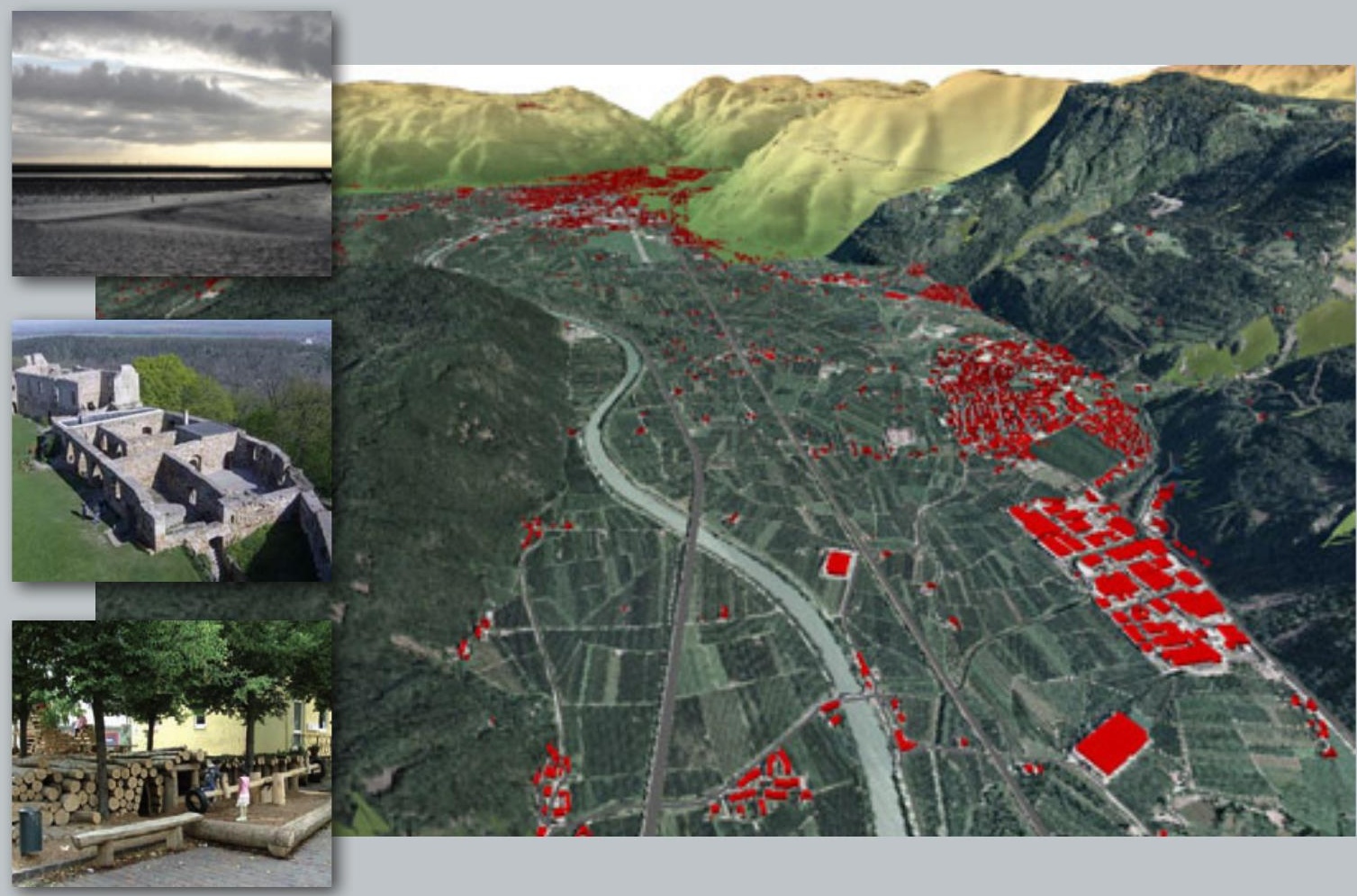



Tobias Reeh, Gerhard Ströhlein, Axel Bader (Hg.)

Kulturlandschaft verstehen

This work is licensed under the Creative Commons License 2.0 "by-nd", allowing you to download, distribute and print the document in a few copies for private or educational use, given that the document stays unchanged and the creator is mentioned. You are not allowed to sell copies of the free version. 
erschienen in der Reihe der Universitätsdrucke

im Universitätsverlag Göttingen 2010 
Tobias Reeh, Gerhard Ströhlein, Axel Bader (Hg.)

\section{Kulturlandschaft verstehen}

ZELTForum - Göttinger Schriften zu Landschaftsinterpretation und Tourismus - Band 5

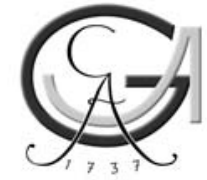

Universitätsverlag Göttingen 2010 


\section{Bibliographische Information der Deutschen Nationalbibliothek}

Die Deutsche Nationalbibliothek verzeichnet diese Publikation in der Deutschen Nationalbibliographie; detaillierte bibliographische Daten sind im Internet über $<$ http://dnb.ddb.de $>$ abrufbar.

Zentrum für Landschaftsinterpretation und Tourismus (ZELT) e.V.

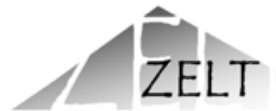

In Kooperation mit dem Geographischen Institut der Georg-August-Universität Göttingen,

Abteilung Humangeographie

Goldschmidtstr. 5

37077 Göttingen

Internet : www.zelt-goettingen.de

Der Druck des Bandes wurde von der Sparkasse Duderstadt und vom Zweckverband Verkehrsverbund Süd-Niedersachsen unterstützt.

\section{Sparkasse Duderstadt}

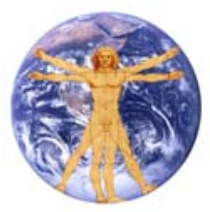

Dieses Buch ist auch als freie Onlineversion über die Homepage des Verlags sowie über den OPAC der Niedersächsischen Staats- und Universitätsbibliothek (http://www.sub.uni-goettingen.de) erreichbar und darf gelesen, heruntergeladen sowie als Privatkopie ausgedruckt werden. Es gelten die Lizenzbestimmungen der Onlineversion. Es ist nicht gestattet, Kopien oder gedruckte Fassungen der freien Onlineversion zu veräußern.

Redaktion: Thomas Wieland Umschlaggestaltung: Franziska Lorenz

Umschlagbilder: Ines Fleer, Kira Gee, Sebastian Schäfer, Urte Stobbe, Thomas Wieland

Die Verantwortung für den Inhalt liegt bei den jeweiligen Autorinnen und Autoren.

C 2010 Universitätsverlag Göttingen

http:/ / univerlag.uni-goettingen.de

ISBN: 978-3-941875-33-3 


\section{Inhalt}

Vorwort

Tobias Reeh, Gerbard Ströblein, Axel Bader

Umweltgeschichte und Kulturlandschaftsforschung eine Einführung

Manuela Armenat, Axel Bader, Jessica Preutenborbeck

Umweltgeschichte durch starke Disziplinen - Vom richtigen und falschen Einsatz der Interdisziplinarität

Max Krott

Interdisziplinarität in der Umweltforschung - Erfahrungen aus der Praxis

Achim Daschkeit

Eine historisch-ökologische Sicht auf Landnutzungsgeschichte und Ökosystementwicklung

Mattbias Bürgi

Historische Wassermühlen in Südniedersachsen

Esben Eidevik

Landschaftswandel und Wahrnehmung - Das Beispiel

Offshore-Windkraft

Kira Gee

Nutzungswandel historischer Gartenanlagen am Beispiel der aktuellen Debatte über den Park Kassel-Wilhelmshöhe

Urte Stobbe

Kriegsbewirtschaftung als Faktor eines Landnutzungswandels -

Das Beispiel der Eichenschälwälder vor und während des

Ersten Weltkrieges

Axel Bader

Der Wald als Psychotop im Spannungsfeld multifunktionaler Landnutzung - das Fallbeispiel Ostalb

Christoph Riegert, Ines Fleer

Künstliche Beschneiung als Anpassungsstrategie an die

Klimaerwärmung und die Nachfrage im Wintertourismus?

Eine Fallstudie zur Sicht der Touristen

David Gallati 



\section{Vorwort}

Tobias Reeh, Gerhard Ströhlein, Axel Bader 


\section{Kulturlandschaft verstehen}

Viele Tourismusregionen streben im Rahmen von Differenzierungsstrategien ein eigenständiges Profil an, um über ein marktfähiges Alleinstellungsmerkmal zu verfügen. Die Kulturlandschaft in ihrer jeweils besonderen Eigenart bietet hierfür ein endogenes Potenzial, das es zu erschließen, aber gleichermaßen auch zu schützen gilt. Die große und mittlerweile globale Bedeutung, die diesem Thema zugemessen wird, zeigt sich nicht zuletzt in den UNESCO-Weltkulturerbelisten, in die auch herausragende Kulturlandschaften aufgenommen werden (vgl. Deutsche UNESCO-Kommission e.V. 2009a, 2009b).

Eine grundlegende Herausforderung bei der Inszenierung dieser Kulturlandschaften besteht darin, dass sich viele Charakteristika und Besonderheiten nicht unmittelbar eröffnen, sondern erst herausgearbeitet und für den Laien ,interpretiert' werden müssen. Dies gilt insbesondere für konkrete historische Kulturlandschaftselemente (z. B. Verkehrswege, architektonische Zeugnisse oder Flurformen), in denen ,geschichtliche Informationen ähnlich wie in einer schriftlichen Archivalie überliefert [werden]“ (Andres \& Schenk 2002, S. 67).

Vor diesem Hintergrund beinhaltet die vorliegende Publikation im ersten Teil ausgewählte Beiträge aus dem Workshop des Graduiertenkollegs „Interdisziplinäre Umweltgeschichte“ zum Thema „Land von Menschenhand - Landnutzung im Wandel der Zeit“. Dieser Workshop fand am 07. und 08. Februar 2008 am Geographischen Institut der Georg-August-Universität Göttingen statt. Dabei wurde das Thema Landnutzungswandel aus der Perspektive verschiedener wissenschaftlicher Fachrichtungen diskutiert. Im Kern ging es um die Aufgabe, die Andres und Schenk (2002, S. 70) folgendermaßen beschreiben: „Die über Jahrtausende gewachsene Kulturlandschaft ist also ein immer wieder beschriebenes Buch, ein Palimpsest, aus dem [...] wichtige Informationen über vergangene Umwelten [herausgelesen werden können]“"

Der zweite Teil enthält praxisbezogene Fallbeispiele, wie Ergebnisse der Kulturlandschaftsforschung einem interessierten Laien-Publikum vermittelt werden können, getreu dem Goethe’schen Motto: „Man sieht nur, was man weiß“. Angesprochen ist damit die Kernkompetenz der Landschaftsinterpretation, versteht sich diese doch als eine Übersetzung von Forschungsergebnissen ,zum Naturund Kulturerbe aus der Expertensprache in eine Darstellung, die bei einem breiten Publikum in Freizeitstimmung Interesse wecken kann. Dabei überträgt sie nicht nur Fachausdrücke in eine allgemeinverständliche, anschauliche Sprache, sondern ordnet wissenschaftliche Fakten in übergeordnete Zusammenhänge ein." (Gee et al. 2002, S. 46). 
Der Band 5 des ,ZELTForum - Göttinger Schriften zu Landschaftsinterpretation und Tourismus" möchte eine Brücke schlagen zwischen der Betrachtung der Kulturlandschaft als wissenschaftlichem Forschungsobjekt auf der einen und als Lernort im Kontext der Freizeitgestaltung auf der anderen Seite.

Die Herausgeber wünschen allen Leserinnen und Lesern eine anregende Lektüre und möchten den Autorinnen und Autoren für ihre Beiträge danken, die unterschiedliche Perspektiven auf das Thema Kulturlandschaft ermöglichen.

Abschließend sei erwähnt, dass der vorliegende Band ohne die freundliche Unterstützung der Sparkasse Duderstadt und des Zweckverbandes Verkehrsverbund Süd-Niedersachsen nicht hätte realisiert werden können. 


\section{Literatur und Quellen}

Andres, W. u. Schenk, W. (2002): Kulturlandschaft - Archive der Vergangenheit oder Wirtschaftsräume der Gegenwart? In: Ehlers, E. u. Leser, H. (Hrsg.): Geographie heute - für die Welt von morgen. S. 67-74. Gotha, Stuttgart: Klett-Perthes.

Deutsche UNESCO-Kommission e.V. (2009a): UNESCO-Liste des Welterbes. URL: http://www.unesco.de/350.html?\&L $=0<08.12 .09>$

Deutsche UNESCO-Kommission e.V. (2009b): Übereinkommen zum Schutz des Kultur- und Naturerbes der Welt.

URL: http://www.unesco.de/ 650.html?\&L $=0<08.12 .09>$

Gee, K., Glawion, R., Kreisel, W. u. Lehnes, P. (2002): Landschaft - kein Buch mit sieben Siegeln. Landschaftsinterpretation entschlüsselt das Natur- und Kulturerbe auf unterhaltsame Weise. In: Ehlers, E. u. Leser, H. (Hrsg.): Geographie heute - für die Welt von morgen. S. 46-56. Gotha, Stuttgart: KlettPerthes. 


\section{Umweltgeschichte und Kulturlandschaftsforschung - eine Einführung}

Manuela Armenat, Axel Bader, Jessica Preutenborbeck 


\begin{abstract}
The term "cultural landscape" can mean different things in different disciplines. In fact, the usage is so varied that it often makes exchanges impossible. This essay is presenting the perspectives of geography, landscape ecology and history. In the natural sciences, the term is taken to mean an area used by humans. The field of cultural studies, however, is still to define the term clearly. Although there are many ties between the separate sciences, humanistic questions of perception and symbolism have largely been ignored to date. The deeper understanding that culture is indefinable without space but also that space cannot be ascertained or construed without culture is illustrative of just how tightly they are interlocked.

Consequently, "cultural landscape" should not only be described superficially but also understood in terms of its symbolism, its image program. This requires the further investigation of the relationship between humans and the environment, the genesis of cultural landscapes, a heuristic approach and a highly interdisciplinary mode of practice. However, if this is to work, a common definition is essential.
\end{abstract}

\title{
Einleitung
}

Den Termini „Landschaft“ und „Kulturlandschaft" werden in den verschiedenen Disziplinen unterschiedliche Bedeutungen zugewiesen. Die Verwendungsformen sind so vielfältig, dass ein unproblematischer Austausch nicht immer möglich ist. Im Folgenden soll Kulturlandschaftsforschung in den Fachbereichen der Landschaftsökologie, Geschichtswissenschaft und Geographie beleuchtet werden.

Kulturlandschaften werden in der Landschaftsökologie und Geographie im Sinne von Nutzlandschaften verstanden. Dabei stellt die Kulturlandschaft im allgemeinen Verständnis den gesamten Raum dar, der vom Menschen beeinflusst ist. Genauer gesagt ist unter Kulturlandschaft nach Burggraaff (1996) ein vom Menschen durch seine gesellschaftlichen, ökonomischen und kulturell-ästhetischen Anforderungen an Landschaft überprägter Naturraum zu verstehen. Er betont dabei die treibenden Faktoren und Prozesse im Kulturlandschaftswandel, die sich erst durch eine weit reichende historische Analyse interpretieren lassen (Burggraaff 1996, S. 10f; außerdem: Konold 1996, S. 123; Bürgi et al. 2004; Schenk 2002). Diesem Verständnis folgend zählen auch überwiegend versiegelte Industrieräume oder vollkommen naturferne Agrarsteppen zur Kulturlandschaft. Nach der Betrachtungsweise der anwendungsbezogenen Felder Landespflege, Landschaftsplanung und Naturschutz wird unterschieden zwischen der historischen Kulturlandschaft und der reinen Produktionslandschaft (Succow 2004, Wöbse 2002). In der historischen Kulturlandschaft sind z.B. Elemente agrarischer, 
vorindustrieller Landnutzungen erhalten und die Nutzung des Menschen ist ökologisch und kulturell überwiegend der Landschaft angepasst (Wöbse 2001). Die Flächen der Produktionslandschaft hingegen sind vollkommen von intensiver menschlicher Nutzung überformt.

In der deutschsprachigen Geschichts- und Kulturwissenschaft wurde der Landschaft in den vergangenen Jahrzehnten relativ wenig Forschungsinteresse entgegen gebracht, wenn man von einer Landes- und Regionalgeschichte einmal absieht. Aber auch sie war eher ein Versuch der regionalen Eingrenzung des Forschungsgegenstandes als ein besonderer Fokus auf die „Landschaft“ selbst. Der Grund für diese auffallende Zurückhaltung ist wohl in der nationalsozialistischen Instrumentalisierung der ,Kulturraumforschung ${ }^{6}$ zu suchen. Erst in den letzten Jahren wurde damit begonnen, ,Landschaft ${ }^{6}$ kulturwissenschaftlich zu erfassen. Bisher ist das vor allem im angelsächsischen Sprachraum geschehen: Meinigs Sammelband „The Interpretation of ordinary landscapes“ enthält neun Aufsätze von Kulturgeographen und zeigt den Forschungsstand bis etwa Mitte der 1970er Jahre auf. Groth und Bressi (1997) stellten einen kulturwissenschaftlichen Analyserahmen für , normale' Landschaften vor. Generell basieren diese angloamerikanischen Ansätze auf theoretischen Arbeiten von Sauer (1963), Zelinsky (1973), Lefebvre (1974), Yi-Fu Tuan (1977) und Jackson (1989).

Auch der Begriff „Kulturlandschaft“" wurde in den deutschsprachigen Kulturwissenschaften noch nicht analytisch klar herausgearbeitet. Zahllos sind dagegen die Aufrufe der letzten Jahre, die Örtlichkeit eines jeden Geschehens doch mitzudenken. So zahllos die Rufe, so eindrucksvoll die Argumente, dass man schon vor einiger Zeit eine räumliche Wende konstatierte. Dieser „spatial turn“ trug ein Raumbewusstsein in die Kulturwissenschaft, besonders in die Geschichte. Dabei war die Richtung klar: Kultur sei ohne Raum schlechterdings nicht erklärbar! Damit ist aber keine historisierende oder kulturwissenschaftliche Erfassung einer bestimmten Landschaft gemeint. Eine Erfassung der Landschaft mit kulturwissenschaftlichen Methoden und Erkenntnisinteressen ist hier noch nicht erfolgt. Dieses Desiderat wurde jedoch erkannt und in den nächsten Monaten werden einige Ansätze präsentiert werden (Kirchhoff u. Trepl 2009). Auch wurden jüngst einige einschlägige Konferenzen abgehalten (20.-21.11.2008 Mainz: Jahrestagung der Arbeitsgemeinschaft Geschichte und EDV; 30.10.-01.11.2008 Berlin: Transcultural Spaces: Challenges of Urbanity, Ecology, and the Environment in the New Millennium).

Allein dieser kurze Überblick der möglichen Herangehensweisen an „Kulturlandschaft" siderat einer gemeinsamen Begrifflichkeit besteht. Diese fehlende gemeinsame Definition von „Kulturlandschaft“ lässt allein schon vermuten, dass die Verschneidung verschiedener Disziplinen bisher noch nicht ausreichend erfolgt ist. 


\section{Kulturlandschaftsforschung - drei Zugänge}

Die derzeitigen Forschungsschwerpunkte der hier im Zentrum stehenden Disziplinen stellen sich wie folgt dar: Im Fachbereich Landschaftsökologie haben Projekte, die im Bereich der Kulturlandschaftsforschung und -geschichte anzusiedeln sind, zumeist einen konkreten Anwendungsbezug oder spannen zumindest den Bogen zur Analyse heutiger Landschaftszustände. Die Betrachtung historischer Landschaftszustände wird häufig mit Aspekten der Naturschutz- und Landschaftsplanung, wie der Analyse und Bewertung von Naturraumpotentialen verknüpft und unter dem Begriff der historisch-ökologischen Landschaftsanalyse eingeordnet. Doch auch Projekte, die der Generierung von reinem Orientierungswissen zur Entwicklung und Geschichte einer Kulturlandschaft dienen, wurden und werden bearbeitet. Beispielhaft hierfür kann die Fallstudie zur Naturnäheentwicklung einer Landschaft von Jansen et al. (2008) genannt werden.

Die Erforschung der Kulturlandschaftsentwicklung erfolgt in der Landschaftsökologie zumeist in Form einer Inventarisierung der belebten und unbelebten Natur in ihren vom Menschen unterschiedlich beeinflussten Zuständen durch die Zeit. In Projekten mit aktuellem Anwendungsbezug steht dabei der Raumbezug im Vordergrund. Die Auswertung und Interpretation der gewonnenen Daten erfolgt entsprechend durch die Anwendung von Landschaftsbilanzen und -analysen sowie die Modellierung von Prozessen mit Hilfe von Geographischen Informationssystemen. Als Ergebnis stehen am Ende zum Beispiel Vorschläge zur Erstellung von Pflege- und Entwicklungsplänen für Arten und Biotope, deren Vorkommen an historische Kulturlandschaftselemente oder -nutzungen gekoppelt ist, Managementpläne, die zur Lösung von aktuellen Nutzungskonflikten auf Landschaftsebene dienen oder auch Prognosen zur Änderung von Standortfaktoren unter spezifischen Nutzungseinflüssen. Des Weiteren ist die Entwicklung von Entscheidungsunterstützungssystemen zur Optimierung von Landnutzungen und als Werkzeug nachhaltiger Landnutzungsplanung (Schönhart et al. 2008, Herzig 2007, Herzig u. Duttmann 2002) ein gegenwärtiger Forschungsschwerpunkt. Aktuelle Arbeiten aus dem Fachbereich Landschaftsökologie, die auf eine theoretische Ebene der kulturgeschichtlichen Interpretation von Landschaftsfunktionen und deren Wahrnehmung sowie ökologische Theorien insbesondere in historischer Zeit abheben, sind eher selten. Ein Beispiel hierfür bieten Kirchhoff und Trepl (2008) mit ihrem Band zu „Landschaft, Wildnis und Ökosystem“.

Die in aktuellen Projekten der Kulturlandschaftsentwicklung und -geschichte angewandten Auswertungsmethoden sind zwar überwiegend naturwissenschaftlich einzuordnen, bei der Erhebung der Daten für historische Landschaftszustände ist jedoch eine Einarbeitung in geisteswissenschaftliche Methoden und der Rückgriff auf das Handwerkszeug eines Historikers, wie zum Beispiel der Umgang mit archivalischen Quellen, unumgänglich. Hinzu kommen sozialwissenschaftliche Aspekte, wenn Ergebnisse in die Regionalentwicklung integriert werden sollen und die Zusammenarbeit mit der Bevölkerung und Behörden erforderlich ist. 
Dementsprechend ist Projekten zur Kulturlandschaftsgeschichte, die dem Fachbereich der Landschaftsökologie entspringen und sich zumeist mit einer breiten Palette an Gesichtspunkten der Kulturlandschaftsentwicklung beschäftigen, heute häufig eine interdisziplinäre Ausrichtung zu eigen. Dabei kann die Interdisziplinarität sowohl in einer Zusammenarbeit mit anderen Fachbereichen bestehen als auch in der Aneignung von fachbereichsfremden Methoden. Aktuell geht der Trend eindeutig zur Bildung von Forschergruppen verschiedener Disziplinen und Fachbereiche. Für das Verständnis der Landschaft als interdisziplinärem Forschungsgegenstand und die Förderung wirklicher Interdisziplinarität durch einen ebensolchen Aufbau von Forschergruppen plädieren auch Ganzert et al. (2007): Die im Themenfeld ,nachhaltige Landnutzung“ zu bearbeitenden ökologischen, sozialen, ökonomischen und institutionellen Problemstellungen seien nur von ökologischen und sozioökonomischen Fachrichtungen gemeinsam lösbar (S. 24).

Entsprechend des Trends, landschaftsökologische Forschung insbesondere im Kontext der Kulturlandschaftsgeschichte verstärkt mit interdisziplinärem Ansatz zu betreiben, verschieben sich die angestrebten Forschungsschwerpunkte in Richtung sozial- und geschichtswissenschaftlich beeinflusster Forschungsfelder. Dies schlägt sich zum einen in einer vermehrten Einbindung von geschichtswissenschaftlichen Methoden (z.B. Oral History) in historisch-ökologische Landschaftsanalysen nieder, zum anderen erfährt die Aufarbeitung der theoretischen Hintergründe der Kulturlandschaftsgeschichte Vorschub (z.B. Anders 2007). Auch im Bereich des Landschaftswandels gewinnen neben dem Einsatz modernster Visualisierungsprogramme zur Rekonstruktion von Landschaftszuständen Aspekte der Landschaftswahrnehmung und -ästhetik immer mehr Gewicht.

Die Verbindung zwischen dem Fachbereich Landschaftsökologie und der Umweltgeschichte wird besonders deutlich, wenn man den aufkommenden Begriff der Historischen Ökologie näher betrachtet, dessen wichtigstes Instrument, die historisch-ökologische Landschaftsanalyse bereits als eine in der landschaftsökologischen Forschung etablierte Methode genannt wurde.

Die Erforschung der Landschaft sowie die Betrachtung des Verhältnisses des Menschen zum Raum stehen in einer langen Tradition der Geographie. Sie sieht sich als eine beschreibende, analysierende und erklärende Wissenschaft des Raumes. Die Historische Geographie hat seit den 1930er Jahren ihre Forschungsmethoden immer weiter entwickelt, um die Veränderungen des Raumes als Ausdruck raumwirksamer Prozesse zu verstehen. Dabei ist sie als Teildisziplin der Geographie in ihrer Betrachtungsweise durchgehend interdisziplinär. Die Rekonstruktion historischer Querschnitte war bis in die 40er Jahre des 20. Jh. hinein weitgehend auf die Analyse und Auswertung von archivalischen Quellen und alten Karten beschränkt. Mit den seit den 1950er Jahren hinzugetretenen Geländeuntersuchungen von Altlandschaftsrelikten wurde für die Kulturlandschafts- und Siedlungsforschung eine spezifisch geographische Arbeitsweise etabliert (vgl. Denecke 2005, S. 17). Diese historisch-geographische Landesaufnahme stellt auch heute noch einen wichtigen Arbeitsansatz für die historisch-geographische Kulturlandschaftsfor- 
Umweltgeschichte und Kulturlandschaftsforschung

schung dar. Neben der eher statischen Betrachtung einzelner Zustände (Querschnitte), werden durch die dynamisch-funktionale Betrachtung (Längsschnitte) das Beziehungsgefüge und die Prozesse in ihren funktionalen, politischen und sozialen Zusammenhängen aus genetischer Perspektive (retrospektiv) analysiert (vgl. Denecke 2005, S. 36ff.; Denecke 1997, S. 37f.)

Nach Denecke haben sich die raumwirksamen Prozesse also sowohl im Raum selbst niedergeschlagen und sind dort fassbar, als auch in den zeitgenössischen Quellen, in denen die wesentlichen Planungen, Entscheidungen und Maßnahmen einer Raumorganisation festgehalten sind. Essentiell sind hierbei in beiden Bereichen die raumbezogenen Fragestellungen. Die Herangehensweise und die Untersuchung der raumwirksamen Prozesse basiert auf drei Fragekomplexen, wobei die Basis die Ermittlung der beteiligten Akteure und Träger, ihr Einfluss und das Zusammenspiel, bilden. Hinter Ihnen stehen die Fragen zu den Grundsätzen, Zielen und Ideologien. Zur Raumgestaltung selbst ist es wesentlich, Fragen nach der Art und Weise und den verwendeten Instrumentarien und durchgesetzten Maßnahmen zu stellen (vgl. Denecke 2005, S. 38f.)

Nach Schenk (2005) bilden heute vier Forschungsfelder der Kulturlandschaftsforschung den Zugang zum Verständnis des Raumes. Das wohl klassischste Forschungsfeld stellen die Historische Geographie und die Genetische Kulturlandschaftsforschung mit den Untersuchungen der „Elemente und Strukturen als Informationsträger vergangener Prozesse der Raumgestaltung“ (Schenk 2005, S. 17) dar. Sie bieten mit ihren Ergebnissen eine Grundlage für das Forschungsfeld der Landschaftsplanung und Landschaftspflege. Durch die normative Aufladung der Kulturlandschaften wirken diese als identitätsstiftend. Die daraus resultierenden spezifischen Verständnisse unterscheiden sich aber oft grundlegend von der Betrachtung und Konnotation aus den wissenschaftlichen Disziplinen und der Fachpolitik (vgl. Schenk 2008, S. 273). Die erweiterte Betrachtung des MenschUmwelt-Verhältnisses bedarf nach Ansicht Schenks eines heuristischen Ansatzes und einer stark interdisziplinär ausgerichteten Arbeitsweise. Dieser Ansatz bedingt die Verknüpfung einer natur- und kulturgeographischen Landschaftsforschung mit anderen Disziplinen.

Ein bedeutender Bereich der Kulturlandschaftsforschung lag bisher eher im Feld der Kulturwissenschaften: die Konstruktion und Wahrnehmung der „(Kultur-)landschaften“. In den vergangenen Jahren wurde diese Ebene in den Raumwissenschaften als Desiderat erkannt und als Erweiterung der dynamischfunktionalen Betrachtung entdeckt (vgl. Schenk 2005, S. 17).

Werden diese Forschungsfelder miteinander verknüpft, wäre der Ansatz einer Kulturlandschaftsforschung aus historisch-geographischer Perspektive, einer auf den Landnutzungswandel bezogenen Umweltgeschichte sehr ähnlich. Sie verdeutlicht aber auch die große Breite von Herangehensweisen und Schwierigkeiten. So betrachtet die Umweltgeschichte zum Einen die Wechselbeziehungen zwischen Menschen und dem Rest der Natur in der Vergangenheit (nach Beinart u. Coates 1995, S. 1), und rekonstruiert zum Anderen die Umweltbedingungen in der Ver- 
gangenheit sowie die Wahrnehmung und Interpretation damals lebender Menschen (vgl. Herrmann 1996, S. 21 ff.; Sieferle 1997, S. 17). Die Kulturlandschaftsforschung der Geographie als Raumwissenschaft deckt mit ihren speziellen Methoden und Herangehensweisen einen bedeutenden Teil für das Verständnis des Mensch-Umwelt-Verhältnisses ab (vgl. Denecke 2005, S. 298), kann aber mit einem erweiterten Blick ihr Potential erhöhen.

Ein wesentlicher Beitrag der Geschichtswissenschaften zur Kulturlandschaftsforschung bestand bisher in einer theoretischen und ikonographischen Analyse von Landschaftsbildern. Dem spatial turn liegt die Annahme zugrunde, Kultur wäre ohne den Ort, die Landschaft nur unvollständig erklärbar. Das ist auch ohne weiteres umkehrbar. Eine solche (also nochmals gewendete) Perspektive drängt sich geradezu auf und formt sich dann doch zu einer selten gestellten Frage: Ist denn der Raum ohne die Kultur denkbar, erfassbar, erklärbar? Anders gefragt: Offenbart sich eine beliebige Kulturlandschaft nicht als eine recht bildhafte Art, die Umgebung darzustellen, zu strukturieren und $\mathrm{zu}$ symbolisieren? Ist ein Landschaftspark tatsächlich, echter', ,wirklicher' als ein Landschaftsgemälde? Eins ist sicher: Er ist greifbarer, erlebbarer. Man kann ihn betreten, fühlen und riechen. Er spricht mehr Sinne in uns an als ein Gemälde. Aber ist er nicht mit den gleichen Methoden verstehbar?

An dieser Stelle soll versucht werden, Landschaft als Bildnis und Symbol zu konzeptualisieren. Denn Landschaften sind nicht einfach nur die Orte, an denen Ereignisse stattfinden. Sie werden vielmehr imaginiert, konstruiert, auf ganz unterschiedliche Weise wahrgenommen und ebenso vielfältig genutzt. So soll nicht nur eine gemeinsame Diskussionsbasis zwischen Geo- und Kulturwissenschaften entstehen, sondern explizit das in einer kulturwissenschaftlichen Erfassung steckende Potential einer Landschaftsanalyse vorgestellt werden. Aus dieser Perspektive bietet sich das Konzept der Ikonologie an. Damit ist die theoretische und historische Erörterung symbolischer Bilder gemeint, die sich nach der hier präsentierten These in einer „Landschaft“ finden lassen.

Als Ikonologie bezeichnet man eine in den 1920er und 1930er Jahren entstandene Forschungsrichtung der Kunstgeschichte, die im „Dunstkreis“ des Hamburger Warburg Instituts entstand. Sie stellt eine Ergänzung zur bis dahin benutzten wertindifferenten Formanalyse und der Ikonographie, die die symbolischen Formen eines Kunstwerks deutet, dar.

Die ikonologische Methode wurde erstmals von Warburg in seiner Straßburger Dissertation von 1892 über zwei Bilder Botticellis durchgeführt. Der Begriff ,,ikonologische Analyse“ taucht zum ersten Mal in seinem Vortrag über die Monatsbilder im Palazzo Schifanoia in Ferrara auf. Ihm gelang es, das komplexe astrologische Bildprogramm der Fresken zu entschlüsseln. Panofsky entwickelte die Ikonologie 1939 weiter zu einem Dreistufenschema der Interpretation:

- die präikonographische Analyse, die Panofsky als eine Analyse von Stil und Form verstand, 
- die ikonographische Analyse, die die Art und Weise, wie unter wechselnden historischen Bedingungen bestimmte Themen oder Vorstellungen durch Gegenstände und Ereignisse ausgedrückt wurden, untersuchte und

- die ikonologische Interpretation.

Bei der ikonologischen Interpretation geht es darum, ein Werk aus seinem Entstehungskontext heraus zu erklären und sein Bildprogramm zu analysieren. Kunstgeschichte verwandelt Visuelles in Text. Kunstwerke werden demnach als verschlüsselte Texte angesehen, die nur von denen verstanden werden können, die die kulturellen Bedingungen ihrer Entstehungszeit kennen. Panofsky wollte mit der ikonologischen Methode die intrinsische Bedeutung eines Kunstwerkes offenlegen. Dazu müsste man allerdings über die „Vertrautheit mit den wesentlichen Tendenzen des menschlichen Geistes, geprägt durch persönliche Psychologie und ,Weltanschauung““ (Panofsky 1975, S. 50) verfügen. Korrektivprinzip sei die „Geschichte kultureller Symptome oder ,Symbole“ allgemein“ (ebd.). Damit meinte er die „Einsicht in die Art und Weise, wie unter wechselnden historischen Bedingungen wesentliche Tendenzen des menschlichen Geistes durch bestimmte Themen und Vorstellungen ausgedrückt wurden“ (ebd.).

In diesem Sinne sollen Formen und Motive, ganze Kunstwerke oder eben Kulturlandschaften als Manifestationen zugrunde liegender Prinzipien aufgefasst werden. Damit würden diese Elemente als etwas interpretiert, das Cassirer (1990) als „symbolische Werte“ bezeichnet hat. Panofsky (1998) selbst wendete seine Methode erstmals in einem Aufsatz über „Die Perspektive als ,symbolische Form““ an. Panofsky versuchte auch den Anwendungsbereich seiner Methode zu erweitern. Er untersuchte gotische Kathedralen und ,las`ihre Architektur als Text. Die Kathedralen von Caen und Durham sollten als kulturelle Symbole ihres Zeitalters ,gelesen“ werden, als ,steingewordene Idee“ und architektonische Scholastik (vgl. Panofsky 1989). Die Brille, als Text' erwies sich auch in anderen Kulturwissenschaften als blickschärfend (vgl. Bachmann-Medick 2006). Schon Panofsky (1975, S. 42) hatte die Anwendung seiner Methode auch in der Ethnologie vorgeschlagen. Geertz konzeptionalisierte ,Kultur als Text' in seiner ,dichten Beschreibung'. Kultur versteht Geertz (1987, S. 9) in Anlehnung an Max Weber als „Bedeutungsgewebe“. „Ihre Untersuchung ist dabei keine experimentelle Wissenschaft, die nach Gesetzen sucht, sondern eine interpretierende, die nach Bedeutungen sucht“" (ebd.). Auch wenn sich Geertz nie explizit auf Panofsky bezog, ähneln sich doch ihre Vorgehensweisen.

Darnton (1986) versuchte in „Le grand massacre des chats: attitudes et croyances dans l'ancienne France" unter anderem, eine religiöse Prozession in Montpellier als Repräsentation der ständischen Ordnung der Stadt zu erklären. In den Jahren vor der Französischen Revolution erschien jedoch den meisten Bewohnern Montpelliers diese Repräsentation als archaisch. Man könnte nun diskutieren, ob dieser Artikel eher der ikonographischen oder der performativen Wende zuzuordnen wäre. Wichtig erscheint an dieser Stelle jedoch vor allem, dass Darnton erst- 
malig versuchte, eine sich modernisierende städtische Landschaft ikonologisch zu analysieren.

Darnton hatte aber einen Vorgänger: den viktorianischen Kunsthistoriker Ruskin. Ruskin behandelte Landschaftsgemälde als Text, die mit einer Art Bibelexegese interpretiert werden könnten. Er versuchte damit eine gewisse Ordnung in das ihm so erscheinende Chaos der frühen Industrialisierung in England zu bringen (vgl.Cosgrove 1979). Damit ordnete er die auf den Gemälden dargestellte ,Landschaft ${ }^{\natural}$ in einem weiteren Kontext als in die Erforschung des Stils und der Form ein. Ruskins Werk wurde in weiten Kreisen rezipiert.

Damit schließt sich der Kreis und die oben aufgeworfenen Fragen scheinen auch hinlänglich beantwortet: ,Raum', ,Landschaft ${ }^{6}$ und ,Landschaftsgemälde“ könnten mit gleichem Instrumentarium analysiert werden. Diese Annahme basiert auf folgender Grundlage: Eine Kulturlandschaft ist eine Landschaft, die durch den Menschen geprägt wurde. Ohne ein tiefgehendes Verständnis der menschlichen Kultur kann sie zwar oberflächlich beschrieben, aber nicht in ihrer Symbolhaftigkeit, in ihrem Bildprogramm verstanden werden. Die Chiffre ,Landschaft als Text ${ }^{6}$, die hier als ein Einstieg vorgestellt wurde, weist zurück auf eine kunsthistorische Analysetradition, die maßgeblich von Panofsky geprägt wurde. Jetzt bleibt nur noch, einige neuere Anwendungsbeispiele aufzuführen: So interpretierte die Militärgeschichte frühneuzeitliche Festungsbauten als gebauten „Herrschaftsanspruch“ (Pröve 1992, S. 321) des Auftrag gebenden Fürsten. Und als ,steinerne Sozialdisziplinierung“ (Pröve 1992, S. 198, 321; Eichberg 1989, S. 534) für die Einwohnerschaft. Diese beiden Beispiele zeigen, dass das Analyseschema auch im deutschsprachigen Raum schon fruchtbar angewandt wurde, freilich bei Architekturen, noch nicht bei einer Landschaft. Das ist zunächst nicht weiter verwunderlich, ist doch ein Bauwerk viel offensichtlicher, von Menschen gemacht ${ }^{6}$ als eine beliebige Landschaft. Und schließlich, so darf hier angenommen werden, hinterließ auch ein Monumentalbau mehr archivalische Quellen als eine Landschaft. Dass sich Historiker - wenn überhaupt - eher Bauwerken als Landschaften zuwandten, lässt sich auch damit erklären.

\section{Zusammenführung}

Es konnte gezeigt werden, dass ein erhebliches Potential einer zukünftigen Kulturlandschaftsforschung in der stärkeren Verschneidung der Forschungsansätze und Methoden verschiedener Disziplinen liegt. Die bisher beteiligten Disziplinen sind vor allem die Geographie und die Landschaftsökologie und -planung. Daneben können in der Forst- und Agrarwissenschaft noch einige Ansätze verortet werden. Auffallend ist bisher eine weitgehende Zurückhaltung der Kulturwissenschaften. Sie ist wohl der nationalsozialistischen Vereinnahmung des Themas als "Kulturraumforschung" zu verdanken. 
Eine Einbeziehung kulturwissenschaftlicher Herangehensweisen offeriert jedoch ein interpretatorisches Instrumentarium, das allen beteiligten Wissenschaften potentiell neue Erkenntnisse über Kulturlandschaften und einen Wandel der Landnutzung liefert. Dazu ist allerdings eine gemeinsame Begriffsverwendung unerlässlich, denn nur sie bietet die Grundlage für eine interdisziplinäre Kommunikation. 


\section{Literatur und Quellen}

Anders, K. (2007): Landschaft und Wald in der Universalgeschichte. Über Orientierungsversuche in Zeiten hoher Dynamik. In: Burkart, B. u. Konold, W. (Hrsg.): Raum-Zeit-Probleme in der Kulturlandschaft. culterra - Schriftenreihe des Instituts für Landespflege der Albert-Ludwigs-Universität Freiburg, Bd. 51, S. 1-15.

Bachmann-Medick, D. (2006): Cultural turns. Neuorientierungen in den Kulturwissenschaften, Reinbeck, S. 70-90.

Bartels, C., Ruiz del Arbol, M., Londen, H. v. u. Orejas, A. (Eds.) (2008): Landmarks profiling europe's historic landscapes. Veröffentlichungen aus dem Deutschen Bergbau-Museum Bochum, Nr. 158, Selbstverlag des Deutschen Bergbau-Museums Bochum.

Beinart, W. u. Coates, P. (1995): Environment and History: The taming of nature in the USA and South Africa. Routlege Publishing. New York.

Burggraaff, P. (1996): Der Begriff „Kulturlandschaft“ und die Aufgaben der „Kulturlandschaftspflege" aus der Sicht der Angewandten Historischen Geographie. In: Natur- und Landschaftskunde 32, 1996, S. 10-11.

Bürgi, M., Hersperger, A. M. u. Schneeberger, N. (2004): Driving forces of landscape change - current and new directions. Landscape Ecology 19, S. 857-868.

Cassirer, E. (1990): Philosophie der symbolischen Formen. Phänomenologie der Erkenntnis, Bd. 3. Darmstadt.

Cosgrove, D. E. (1979): John Ruskin and the geographical imagination. In: Geographical Review 69, S. 43-62.

Darnton, R. (1986): Le grand massacre des chats: attitudes et croyances dans l'ancienne France, Paris. Dt. Übersetzung: Ders.: Das große Katzenmassaker. Streifzüge durch die französische Kultur vor der Revolution, München 1989. Darin: Ders.: Ein Bourgeois bringt seine Welt in Ordnung. Die Stadt als Text. S. 92-125.

Denecke, D. (1997): Quellen, Methoden, Fragestellungen und Betrachtungsansätze der anwendungsorientierten geographischen Kulturlandschaftsforschung. In: Schenk, W., Fehn, K. u. Denecke, D. (Hrsg.): Kulturlandschaftspflege. Beiträge der Geographie zur räumlichen Planung. Gebrüder Bornträger. Berlin/Stuttgart

Denecke, D. (2005a): Entwicklung in der deutschen Landeskunde und Kulturlandschaftsforschung. In: Fehn, K. u. Simms, A. (Hrsg.) (2005): Wege der Historischen Geographie und Kulturlandschaftsforschung. Ausgewählte Beiträge. Zum 70. Geburtstag von D. Denecke. Steiner Verlag. Stuttgart. 
Denecke, D. (2005b): Ausblick: Auswahl der Beiträge, Forschungsansätze und Forschungsentwicklung. In: Fehn, K. u. Simms, A. (Hrsg.) (2005): Wege der Historischen Geographie und Kulturlandschaftsforschung. Ausgewählte Beiträge. Zum 70. Geburtstag von D. Denecke. Steiner Verlag. Stuttgart.

Eichberg, H. (1989): Zentralmacht und Sozialgeometrie. Kriegsingenieurwesen des 17. Jahrhunderts in den Herzogtümern Bremen und Verden. Köln.

Ganzert, C., Kirchner-Heßler, R., Gerber, A. u. Konold, W. (2007): Das Modellvorhaben Kulturlandschaft Hohenlohe: Anlass und Hintergründe. In Kirchner-Heßler, R., Gerber, A. u. Konold, W. (Hrsg.): Nachhaltige Landnutzung durch Kooperation von Wissenschaft und Praxis. oekom Verlag, Kulturlandschaft Bd. I, Teil I, S. 22-31.

Geertz, C. (1987): Dichte Beschreibung. Beiträge zum Verstehen kultureller Systeme. Frankfurt/M.

Geertz, C. (1983): Art as a cultural system. In: Ders. Local knowledge: further essays in interpretative anthropology, New York.

Groth, P. u. Bressi, T. W. (1997): Understanding ordinary landscapes. Ann Arbor.

Hammer, T. u. Leng, M. (2008): Moorlandschaften im Bedeutungswandel. Zur gesamtgesellschaftlichen Aufwertung naturnaher Kulturlandschaften. Schriftenreihe der interfakultären Koordinationsstelle für Allgemeine Ökologie (IKAÖ), Universität Bern, Bd. 10.

Herrmann, B. (1996): Umweltgeschichte als Integration von Natur- und Kulturwissenschaften. In: Bayerl, G., Fuchsloch, N. u. Meyer, T. (Hrsg.): Umweltgeschichte. Methoden, Themen, Potentiale. Münster u.a., Cottbuser Studien zur Geschichte von Technik, Arbeit und Umwelt 1, S. 21-30.

Herzig, A. (2007): Entwicklung eines GIS-basierten Entscheidungsunterstützungssystems als Werkzeug nachhaltiger Landnutzungsplanung. Konzeption und Aufbau des räumlichen Landnutzungsmanagementsystems LUMASS für die ökologische Optimierung von Landnutzungsprozessen und -mustern. Kieler geographische Schriften, Bd. 114.

Herzig, A. u. Duttmann, R. (2002): Entscheidungsunterstützungssysteme als Werkzeuge nachhaltiger Landnutzungsplanung. In: Strobl, J., Blaschke, T. u. Griesebner, G. (Hrsg.): Angewandte Geographische Informationsverarbeitung XIV - Beiträge zum 14. AGIT-Symposium Salzburg, S. 182-187.

Jackson, P. A. (1989): Maps of meaning. London.

Jansen, F., Zerbe, S. u. Succow, M. (2008): Changes in landscape naturalness derived from a historical land register - a case study from NE Germany. Landscape Ecology. 
Kirchhoff, T. u. Trepl, L. (2008): Landschaft, Wildnis, Ökosystem - eine kulturgeschichtliche Analyse ihrer Mehrdeutigkeit In: Technische Universität München, Lehrstühle für Landschaftsarchitektur und Landschaftsplanung (Hrsg.): Ambiguous landscapes - Vieldeutige Landschaften. Bode, Freising. S. 78-83.

Kirchhoff, T. u. Trepl, L. (2009): Vieldeutige Natur. Landschaft, Wildnis und Ökosystem als kulturgeschichtliche Phänomene. Bielefeld.

Lefebvre, H. (1974): The production of space. London.

Meinig, D. W. (1979): The Interpretation of ordinary landscapes. Geographical essays. Austin.

Konold, W. (1996): Von der Dynamik einer Kulturlandschaft. Das Beispiel Allgäu. In: Konold, W. (Hrsg.): Naturlandschaft Kulturlandschaft. Die Veränderung der Landschaften nach der Nutzbarmachung durch den Menschen.

S. 121-136.

Panofsky, E. (1975): Ikonographie und Ikonologie. Eine Einführung in die Kunst der Renaissance. In: ders.: Sinn und Deutung in der bildenden Kunst. Köln. S. 36-64.

Panofsky, E. (1989): Gotische Architektur und Scholastik. Zur Analogie von Kunst, Philosophie und Theologie im Mittelalter, Köln.

Panofsky, E. (1998): Die Perspektive als „symbolische Form“. In: Michels, K., Warnke, M. u. Panofsky, E. (Hrsg.): Deutschsprachige Aufsätze II, Berlin 1998, S. 664-757. Erstmalig in: Saxl, F. (Hrsg.): Vorträge der Bibliothek Warburg 1924-1925, Leipzig/Berlin 1927, S. 258-330.

Pröve, R. (1992): Stehendes Heer und städtische Gesellschaft im 18. Jahrhundert. Göttingen.

Sauer, C. O. (1963): Land and Life. A selection of writings of Carl Ortwin Sauer. Berkeley.

Schenk, W. (2002): „Landschaft“ und „Kulturlandschaft“ - ,getönte“ Leitbegriffe für aktuelle Konzepte geographischer Forschung und räumlicher Planung. Petermanns Geographische Mitteilungen 146, S. 6-13.

Schenk, W. (2005): „Kulturlandschaft“ als Forschungskonzept und Planungsauftrag - aktuelle Themenfelder der Kulturlandschaftsforschung. In: Denzer, V., Hasse, J., Kleefeld, K.-D. u. Recker, U. (Hrsg.): Kulturlandschaft. Wahrnehmung - Inventarisierung - Regionale Beispiele. Selbstverlag Landesamt für Denkmalpflege Hessen. Wiesbaden.

Schenk, W. (2008): Aktuelle Verständnisse von Kulturlandschaft in der deutschen Raumplanung - ein Zwischenbericht. Informationen zur Raumentwicklung, H. 5. 2008, S. 271-277. 
Schönhart, M., Schauppenlehner, T., Schmid, E. u. Tröger, M. (2008): Konzept zur Modell- und Datenintegration für ein Landnutzungsoptimierungsmodell zur Simulation der Auswirkungen regionaler Ernährungsstrategien. In: Strobl, J., Blaschke, N. u. Griesebner, G. (Hrsg.): Angewandte Geoinformatik 2008. Beiträge zum 20. AGIT-Symposium Salzburg. Wichmann Verlag. Heidelberg. S. 134-139.

Sieferle, R.P. (1997): Rückblick auf die Natur. Eine Geschichte des Menschen und seiner Umwelt. Luchterhand. München.

Succow, M. (2004): Kultur- und Naturlandschaften und neue Wildnis. In: Deutsches MAB-Nationalkomitee (Hrsg.): Voller Leben. UNESCO-

Biosphärenreservate - Modellregionen für eine nachhaltige Entwicklung. Berlin und Heidelberg. S. 73-79.

Tuan, Y.-F. (1977): Space and place. The perspective of experience. Minneapolis.

Wöbse, H.-H. (2001): Historische Kulturlandschaften, Kulturlandschaftsteile und Kulturlandschaftselemente. In: Kommunalverband Großraum Hannover (Hrsg.): Kulturlandschaften in Europa - regionale und internationale Konzepte zu Bestandeserfassung und Management. Beiträge zu regionalen Entwicklung Hannover, Bd. 92. S. 9-12.

Wöbse, H.-H. (2002): Landschaftsästhetik. Über das Wesen, die Bedeutung und den Umgang mit landschaftlicher Schönheit. Stuttgart-Hohenheim.

Zelinsky, W. (1973): The cultural geography oft the United States. Englewood Cliffs. 


\section{Umweltgeschichte durch starke Disziplinen - Vom richtigen und falschen Einsatz der Interdisziplinarität}

Max Krott*

* Ich danke den beiden anonymen Gutachtern für ihre hilfreichen Kommentare und Anmerkungen zu einer früheren Version des Artikels. 


\begin{abstract}
In contrast to be used as a catchword only of new management of science interdisciplinarity could contribute to the design of innovative disciplines of environmental history if it is filled with a clear content. Interdisciplinarity can be specified as the phase in research in which one discipline integrates within another one at least partly. The results are new knowledge or even a new innovative discipline. This concept can be useful in designing innovative disciplines which focus on the key issue of environmental history how to cover culture and nature within the historical process. The integrative phase is full of conflicts due to the fact that disciplines compete and not all disciplines participating win. The integration needs professional management of conflicts and additional resources. Keeping in mind that the integration is risky and fails often it becomes clear that interdisciplinarity remains an valuable exceptional phase in environmental history but it is not a concept for day to day research.
\end{abstract}

\title{
Die Frage nach den Grenzen des Allheilmittels „Interdis- ziplinarität"“
}

Umweltgeschichte wird umfassend und damit synonym ,interdisziplinär" konzipiert. Sein Nachdenken über Umweltgeschichte führt Radkau (2000, S. 15) zu der Feststellung, dass „... eine Umweltgeschichte, die diesen Namen verdient, nicht nur von Menschen ... sondern auch von ... Schafen ... handelt“; „...unbeabsichtigte Wirkungsketten menschlichen Handelns, bei denen sich Naturzusammenhänge bemerkbar machen, verdienen besondere Aufmerksamkeit." Seine umfangreichen theoretischen und empirischen Argumente legen dar, wie Umweltgeschichte sowohl im Menschlichen als auch im Ökologischen - und damit Natürlichen - ihren zeitlichen Verlauf nimmt. Diesen komplexen Prozess hoffen Radkau und zahlreiche andere Autoren (u.a. Winiwarter u. Knoll 2007; Brüggemeir 2003; Herrmann 1994) mit einem komplexen Verbund von Disziplinen wissenschaftlich zu erfassen. Die von allen Autoren als unverzichtbar angesehene Einbeziehung naturwissenschaftlicher Disziplinen in die ihrem Ursprung nach geisteswissenschaftliche Umweltgeschichte soll in einer ,interdisziplinären“ Umweltgeschichte gelingen (vgl. Pfister 2007, S. 60).

Hier stellt sich die Frage, ob „Interdisziplinarität“ eine solche, von allen Autoren als schwierig eingeschätzte Aufgabe leisten kann. Ist dieses Konzept überhaupt geeignet, alle mit dem umweltgeschichtlichen Verlauf verbundenen Disziplinen $\mathrm{zu}$ integrieren und wenn ja, welche theoretischen, methodischen und forschungsorganisatorischen Folgen hat das für das Fach Umweltgeschichte? 
Das Interessante an dieser Fragestellung ist ihre weit über die Umweltgeschichte hinausreichende Aktualität. Denn von Interdisziplinarität wird heute in allen Bereichen der Wissenschaft viel erwartet: Kaum ein Forschungsprogramm kommt ohne diese Vokabel aus. Die Europäische Union beschwört damit eine generelle „Verbesserung“ der Projekte. Durch Interdisziplinarität will sie Exzellenz erreichen. Neue Erkenntnisse sollen interdisziplinär schneller zu haben sein und die Fähigkeit zur Lösung der Probleme der Praxis steigen. Ganze Professuren und Zentren sollen auf ,interdisziplinärer Basis“ gegründet werden, um den Forschungsertrag zu erhöhen. Die Programmatik erweckt den Eindruck, „Interdisziplinarität" hilft immer. Dieses in der Praxis des Forschungsmanagements weithin verkündete Konzept soll hier am Beispiel der Umweltgeschichte analysiert werden.

Die Analyse sieht sich einem sozialwissenschaftlichen Ansatz verpflichtet, der Wissenschaft als Handeln von Menschen auffasst und den Informations- und Machtprozessen gleiche Aufmerksamkeit schenkt (vgl. Krott 1994). Methodisch werden zum einen die Konzepte zur Umweltgeschichte in der jüngsten Literatur ausgewertet und zum anderen Erkenntnisse aus 15 Jahren empirischer wissenschaftlicher Begleitforschung inter- und transdisziplinärer Forschungsprogramme als Vergleichsmaßstab verwendet (vgl. Krott 2005). Zusätzlich fühlt sich der Autor durch die forstgeschichtliche Forschung an seinem Lehrstuhl an der GeorgAugust-Universität Göttingen ermuntert, zur konzeptionellen Diskussion der Umweltgeschichte, die von sich aus die Forstgeschichte programmatisch umfassen möchte, beizutragen.

\section{Einfache und komplexe Zugänge zur Umweltgeschichte}

Mit der Verankerung im menschlichen Handeln und in den natürlichen Reaktionen der Umwelt spannen die oben erwähnten Autoren für die Umweltgeschichte einen weiten Rahmen auf. Sie führen überzeugende Beispiele an, wie menschliche Handlungen (z.B. die Überweidung) ökologische Elemente (z.B. den Wald) zerstören und umgekehrt, wie die Erschöpfung ökologischer Ressourcen das Überleben ganzer Staaten bedroht haben. Die Reaktionen der Menschen werden im Alltag, aber auch in Politik und Kultur beschrieben. Die Umwelt ändert sich teilweise autonom und teilweise in Abhängigkeit von den Menschen, wie die historische Klimaforschung belegt. Es erscheint auch dem Autor erwiesen, dass Umweltgeschichte mit natürlichen und menschlichen Entwicklungen und deren Wechselwirkungen zu tun hat. Aber folgt daraus zwingend die Notwendigkeit, Umweltgeschichte mit einer umfassenden Basis von unterschiedlichen Disziplinen wissenschaftlichen zu betreiben?

Als Antwort soll an den Unterschied zwischen dem Forschungsgegenstand und der Forschungsdisziplin erinnert werden. Der Gegenstand ist die umweltgeschichtliche Entwicklung als ein komplexes empirisches Phänomen, aber die For- 
Umweltgeschichte durch starke Disziplinen

schungsdisziplinen, die sich diesem Phänomen zuwenden, unterscheiden sich vom Gegenstand dadurch, dass sie immer nur bestimmte Ausschnitte der Wirklichkeit betrachten (vgl. Defila et al. 2006, S. 33). So ist beispielsweise der Mensch komplex, aber er wird von völlig unterschiedlichen Disziplinen aus der Natur-, Sozial-, Wirtschafts- und Geisteswissenschaft beforscht. Jede hat ihren besonderen Blickwinkel und wird dem ganzen Menschen nicht gerecht, aber dennoch trägt sie Erkenntnisse bei. Es ist fraglich, ob eine umfassende Menschenwissenschaft, die an die Komplexität des empirischen Phänomens heranreicht, überhaupt organisierbar wäre.

Auch in der Umweltgeschichte ist das Argument, die Disziplin muss so komplex werden wie der Gegenstand, nicht zwingend. Eine Allumfassende ,interdisziplinäre" Umweltgeschichte wäre wohl nur schwer organisierbar und methodisch/theoretisch auch nicht produktiv. Das wissen die zitierten Autoren wohl auch, aber ihr Argument geht in die Richtung, immer mehr Disziplinen in die Umweltgeschichte zu integrieren, um deren Analysekraft zu erhöhen. Im Unterschied dazu scheint dem Autor die Kunst, die Umweltgeschichte zu einer leistungsfähigen Disziplin zu machen, darin zu liegen, die Disziplin auf Kernprozesse zu fokussieren, die umwelthistorische Verläufe kennzeichnen und/oder je nach wissenschaftstheoretischem Standpunkt auch erklären können.

Allgemein gesprochen ist es nicht aussichtsreich, nur wegen eines komplexen Gegenstandes nach einem (inter-)disziplinär komplexen Zugang zu suchen. Im Gegenteil: Ein disziplinär einfacher Ansatz lässt sich methodisch und theoretisch einfacher fassen und erzielt daher auch rascher Erkenntnisse. Konkret kann auch eine fokussierte Vorgehensweise, sei es in einer geisteswissenschaftlichen oder in einer naturwissenschaftlichen Disziplin, wichtige Beiträge über umwelthistorische Entwicklungen liefern. Eine naturwissenschaftlich gesicherte Darstellung der Klimaentwicklung hat beispielsweise großen Wert in der Waldgeschichte und Erklärungskraft für die historische Entwicklung der alpinen Wald- und Baumgrenze auch dann, wenn keine kulturwissenschaftlichen Faktoren gleichzeitig betrachtet werden. Innerhalb nur einer Disziplin gelingt vor allem die theoretische Begründung einfacher. Demgegenüber eröffnet die interdisziplinäre Vernetzung die Chance, leistungsfähigere Erklärungen $\mathrm{zu}$ formulieren. Ihre Umsetzung in die Forschungspraxis ist aber ungleich anspruchsvoller und verträgt sich nur schlecht mit dem „Einzelkämpfer“, der den Arbeitsstil des Umwelthistorikers zumeist kennzeichnet (vgl. Pfister 2007, S. 60).

Wollte man, wie Winiwarter und Knoll (2007) argumentieren, einen Brückenschlag zwischen Kultur- und Naturwissenschaften wagen, dann empfiehlt es sich zur Vereinfachung der Aufgabe, eine konkrete naturwissenschaftliche und eine konkrete kulturwissenschaftliche Disziplin auszuwählen und die Verknüpfung auf der Ebene der spezifischen Theorien bzw. Methoden zu suchen. Ein allgemeines Konzept hat dagegen geringe Chancen, theoretisch anspruchsvoll zu gelingen. Die Ursache liegt in der Eigenheit der Disziplinen, je einen bestimmten Ausschnitt des Objektes Umweltgeschichte mit einer spezifischen Methode zu betrachten. Zwei 
Disziplinen zur Deckung zu bringen ist einfacher als drei usw. Ein genereller Anspruch, alle relevanten Disziplinen zu verknüpfen, würde bald an die Grenze der Komplexität stoßen.

Da die Komplexität der Disziplinen hinter der Komplexität des Objektes Umweltgeschichte zurückbleibt und hohe Komplexität rasch zu unlösbaren theoretischen und methodischen Problemen führt, ist das undifferenzierte Bemühen, immer mehr Disziplinen in der Umweltgeschichte zu verbinden, keine empfehlenswerte Strategie. Der Erfolg wird stärker durch die Auswahl der richtigen Disziplinen als durch die Erhöhung ihrer Anzahl bestimmt. Eine Vermehrung der einbezogenen Disziplinen ergibt keineswegs per se bessere Einsicht in die Umweltgeschichte.

\section{Interdisziplinarität diesseits ganzheitlicher Forschung}

Den Wunsch, die komplexe Umweltgeschichte in ihrer Gesamtheit „ganzheitlich“ zu analysieren, kann Interdisziplinarität nicht erfüllen. Mit Defila et al. (2006, S. 33) wird als Interdisziplinarität die Zusammenarbeit von konkreten Disziplinen (mindestens zwei) verstanden. Dabei werden die Grenzen einzelner Disziplinen überschritten, die Forschung bleibt aber auf die konkreten Disziplinen bezogen, zwischen denen sie eine Verbindung herstellt. Eine von den Disziplinen losgelöste Ganzheitlichkeit entsteht nicht.

Die ,ganzheitliche Betrachtung“ stößt im Rahmen einer disziplinär aufgebauten Forschung auf das Paradox: sie wäre selbst nichts anderes als eine Disziplin. Disziplinen sind durch bestimmte (in Theorien beschriebene) Inhalte und durch besondere Methoden gekennzeichnet. Im Rahmen einer sozialwissenschaftlichen Analyse sind in der Definition einer Disziplin noch die Ressourcen zu ergänzen. Jede Disziplin wird von einer Anzahl von Forschern vertreten, die über Sacheinschließlich Finanzmittel verfügen (vgl. Krott 2001). Würden nun Forscher beschließen, sich mit ganzheitlichen Inhalten zu beschäftigen und zu deren Gewinnung geeignete Methoden einzusetzen, dann wären genau jene Bedingungen erfüllt, die eine Disziplin, nämlich die „Ganzheitliche Umweltgeschichte“ begründen. Diese würde sich neben den bestehenden Disziplinen zu etablieren versuchen und damit genauso handeln wie alle Disziplinen, nämlich untereinander in Wettbewerb um Erkenntnisse, Personen und Ressourcen treten. Die ganzheitliche Umweltgeschichte hätte zwar aufgrund ihres sehr umfassenden Anspruchs viel Konkurrenz und durch die erheblichen interne Integrationsprobleme relative wenig Kraft, aber sie wäre genau an jene Erfolgsfaktoren gebunden, die auch für andere Disziplinen gelten. Wie auch immer das Schicksal des Experiments „Ganzheitliche Umweltgeschichte“ ausgehen möge, ihre Ziele und Probleme sind nicht die, die interdisziplinäres Arbeiten kennzeichnen.

Es geht hier nicht um die Bewertung einer möglichen Disziplin „Ganzheitliche Umweltgeschichte“. Wohl aber gilt es zu betonen, dass Interdisziplinarität nicht 
Umweltgeschichte durch starke Disziplinen

mit einer solchen Disziplin ident ist und nicht einmal eine Hilfestellungen leistet, wenn man eine solche aufbauen wollte. Die Unterscheidung des Wunsches nach Ganzheitlichkeit von dem Konzept der Interdisziplinarität ist für eine Diskussion der Bedeutung von Interdisziplinarität für die Umweltgeschichte wichtig.

\section{Umweltgeschichtliche Fachdisziplinen als interdisziplinä- res Ergebnis}

Eine nähere Betrachtung der oben zitierten Konzepte für eine ,interdisziplinäre Umweltgeschichte“ zeigt, dass diese jeweils auf eine spezifische umweltgeschichtliche Fachdisziplin abzielen und nicht einfach ,ganzheitlich umfassend“ Umweltgeschichte konzipieren. Brunner (1997, S. 327) spricht sich für eine Umweltgeschichte als „Mentalitätsgeschichte“ aus. Er möchte diese interdisziplinär anlegen, grenzt aber die Naturgeschichte, d.h. die Naturwissenschaften aus seinem Konzept ausdrücklich aus. Die Grundlage seines Ansatzes ist die ,gesellschaftliche Wahrnehmung“ und damit sehr weit von der Naturwissenschaft, in der die Gesellschaft als handelndes Subjekt nicht vorkommt, entfernt. Die kritischen historischen Methoden sollen genutzt und erweitert werden. Ein solches Konzept fokussiert auf einen speziellen Zugang, der Geschichtswissenschaft mit Textkritik und gesellschaftlichen Wahrnehmungs- und Deutungsanalysen verbindet, und baut darum herum eine neue Fachdisziplin auf.

Winiwarter (2007, S. 134) spricht in ihrem Konzept der Umweltgeschichte von „Erklärung“ und hält es für unerlässlich, dazu die „Naturwissenschaften als Partner" zu gewinnen. Sie versucht diese Aufgabe mit einem Test unterschiedlicher naturwissenschaftlicher Theorien auf deren Eignung für die Verknüpfung mit einer spezifischen Geschichtsforschung, nämlich der Geschichte als besondere „Erzählweise“, zu lösen. Sie prüft unterschiedliche Verknüpfungsangebote und wertet dazu u.a. auch Luhmanns Systemtheorie aus. Diese dürre Skizze genügt, um die Winiwarter'sche Arbeitweise zu erkennen, die weit von einer ganzheitlichen Umfassung und Einbeziehung von immer mehr Disziplinen in eine interdisziplinäre Umweltgeschichte entfernt ist. Im Gegenteil wählt sie nur einige naturwissenschaftliche und sozialwissenschaftliche Disziplinen aus, die ihr für die interdisziplinäre Verknüpfung zu einer neuen Umweltgeschichte geeignet erscheinen.

Das Anliegen, im Ergebnis eine Disziplin Umweltgeschichte zu schaffen, die von anderen Disziplinen der Geschichtswissenschaft, aber natürlich auch von der Wissenschaft insgesamt anerkannt ist, wird auch in den Überlegungen von Freytag (2006) deutlich, der sich die Aufnahme der Umweltgeschichte in den ,geschichtswissenschaftlichen Kanon" wünscht. Wohl scheint ihm die Erforschung der Wechselwirkungen zwischen Mensch und Natur im Mittelpunkt zu stehen, aber diese wird nicht umfassend ,interdisziplinär“ angestrebt sondern auf Grundlage der Geschichtswissenschaft und ausgewählten Verknüpfungen zu Natur- und Sozialwissenschaften. Als fruchtbar wird insbesondere der gelungene „Anschluss 
an grundlegende Methoden und Tendenzen der Geschichts- und Sozialwissenschaften" identifiziert.

Die drei Beispiele zeigen, dass hinter den von den Autoren auch als ,interdisziplinär" bezeichneten Konzepten die Idee steht, eine durch Verknüpfung mit ausgewählten anderen Disziplinen verbesserte Umweltgeschichte zu schaffen, die insbesondere geeignet ist, das Verhältnis Mensch/Natur in den Blick zu nehmen. Die neue Umweltgeschichte wäre aber jeweils ein eigenes neues Fach. Dessen besondere Leistung soll sein, geeignete Theorien und Methoden anzubieten. Damit steht außer Frage, dass neue umweltgeschichtliche (Teil-)disziplinen angestrebt werden, für die die Bezeichnung ,interdisziplinär“ wie oben gezeigt widersinnig wäre. Was aber kann dann der immer erwähnte Zusatz „Interdisziplinarität“ mit den neuen umweltgeschichtlichen Disziplinen zu tun haben?

\section{Interdisziplinarität als Phase für Innovation}

Greift man auf die hier eingeführte Definition von Interdisziplinarität als grenzüberschreitende Zusammenarbeit mindestens zweier Disziplinen zurück, dann wird erkennbar, dass interdisziplinär keine Eigenschaft einer besonderen Disziplin ist sondern ein Merkmal der Zusammenarbeit. Wenn die Phase der Zusammenarbeit beendet ist, dann mündet die Interdisziplinarität wieder in eine durch eben diese Erweiterung verbesserte disziplinäre Erkenntnis. Interdisziplinär wäre dann jene besondere Phase, in der sich die Geschichtsforschung öffnet, um in Zusammenarbeit mit ausgewählten anderen Disziplinen in innovativer Weise den Gegenstand ,umweltgeschichtliche Entwicklung“ zu erforschen.

Innovativ bedeutet, dass sich aus der Zusammenarbeit mit einer anderen Disziplin, zumindest neue Erkenntnisse ergeben. Die eigene Disziplin nimmt eine zusätzliche Variable auf, mit deren Hilfe sich Aussagen mit größerer beschreibender oder erklärender Kraft formulieren lassen. So könnte beispielsweise der naturwissenschaftlich nachweisbare oder nicht nachweisbare Zusammenbruch des Waldökosystems in Deutschland die mentalitätsgeschichtliche Deutung des „Waldsterbens“ differenzierter gelingen lassen, als wenn die Frage nach dem „Realphänomen" offen bleiben muss. Die interdisziplinäre Innovation kann über einzelne Hypothesen hinaus bis zur Entstehung einer neue umweltgeschichtlichen Methodik oder Theorie, die dann auf Grundlage einer der Partnerdisziplinen formuliert wird, reichen.

Interdisziplinarität als Phase der Innovation macht in einer Welt der disziplinär organisierten Wissenschaft Sinn. Dieser Kern der Interdisziplinarität wird verdeckt, wenn wie häufig von ,interdisziplinären Fächern“ gesprochen wird. So verwirrt auch die Redeweise von einer „Interdisziplinären Umweltgeschichte“ den Leser. Er könnte eine „Interdisziplinäre Disziplin“ erwarten, die es, wie gezeigt, gar nicht geben kann. Nur schwer entdeckt er den innovativen Kern, der im Wort 
Interdisziplinarität verborgen ist, der sich auf den Prozess der Zusammenarbeit zwischen Disziplinen und nicht auf das Ergebnis bezieht.

Die interdisziplinäre Zusammenarbeit wird mit neuen Einsichten oder sogar mit dem dauerhaften Ergebnis, eines neuen umwelthistorischen Fachgebiets, belohnt, sie hat aber erhebliche Schwierigkeiten zu überwinden (vgl. Defila et al. 2006). Weil die interdisziplinäre Zusammenarbeit stets kritisch bleibt, erweist es sich als nützlich, den Begriff Interdisziplinarität für die eine Phase, nämlich die Zusammenarbeit zu verwenden und damit nicht das neu entstandene Wissen oder im besonderen Erfolgsfall das neu kreierte Fachgebiet zu belasten. Die neuen umweltgeschichtlichen Fachgebiete werden ihre neuen Methoden ohne größere Konflikte anwenden können und auch an ihren Theorien ohne große Brüche bauen können. Eben dies ist das Versprechen jeder Disziplin und rechtfertig die neuen umweltgeschichtlichen Disziplinen. Interdisziplinär und deshalb konfliktreich und schwierig war lediglich der Anfang.

Die Konflikte in der Interdisziplinarität entstehen unweigerlich aus dem Wettbewerb, in dem alle Disziplinen stehen. Knappe Ressourcen reichen nicht für alle und die Weltsichten der Disziplinen wetteifern um Aufmerksamkeit von Wissenschaft und Praxis. Die interdisziplinäre Zusammenarbeit hebt die Konkurrenz nicht auf, im Gegenteil, sie verschärft sie, weil das konfliktminimierende Nebeneinander durch ein Miteinander ersetzt werden soll, in dem Ausweichen als Option wegfällt. Die Grenzen zwischen (zumindest) zwei Disziplinen werden nicht mehr respektiert und durchdrungen. Beispielsweise gerät die Geologie in die Sicht der gesellschaftlichen Mentalitätsgeschichte, d.h. der ,geologische Granit“ als ein Material mit naturwissenschaftlich objektiv gemessener Härte wandelt sich in eine gesellschaftliche Interpretation, in der eben dieses Material zu einem Granit wird, dem Eigenschaften wie kalt, gefährlich oder Heimat zugeschrieben werden. In diesem interdisziplinären Konflikt kann nur eine Disziplin die Oberhand gewinnen, entweder setzt sich die Geologie durch und die Mehrdeutigkeit des Mentalitätsdiskurses wird in klare Definitionen gezwungen, oder die Mentalitätsgeschichte dient als Basis, dann löst sich die naturwissenschaftliche Klarheit unvermeidlich auf.

Die interdisziplinäre Zusammenarbeit kann sich auf einzelne Erkenntnisse und Begriffe beschränken. Sie kann aber auch soweit gehen, dass eine Disziplin als Basis Teile einer anderen Disziplin in sich aufnimmt. In diesem Fall gewinnt die Basisdisziplin nicht nur an einer Erweiterung der Aussagegegenstände sondern auch an der dadurch erforderlichen Neufassung von Begriffen und Erklärungen. Die andere Disziplin verliert jedoch. Weil Interdisziplinarität kein harmonischer Prozess ist, in dem alle Beteiligten gewinnen, erzeugt sie Konflikte. Deren Überwindung erfordert zum einen professionelles Verbundmanagement und zum anderen sollte der Ertrag den immer erforderlichen hohen Aufwand lohnen. Interdisziplinarität ist daher eine Ausnahmestrategie und eignet sich nicht als Grundlage für den Normalfall der Forschung (vgl. Krott 2001). Auch in der Umweltgeschichte werden die interdisziplinären Phasen die Ausnahmen bleiben und der 
wissenschaftliche Ertrag in der Arbeit der dadurch erneuerten umweltgeschichtlichen Disziplinen anfallen.

\section{Interdisziplinäre Konflikte im Systemansatz}

Professionelles Verbundmanagement zeichnet sich durch eine klar beschriebene Vernetzungsstrategie aus (vgl. Defila et al. 2006, S. 91; Krott 1996). Sehr viele aktuelle interdisziplinäre Vorhaben planen jedoch ihr Vorgehen hauptsächlich implizit und hegen Illusionen über den Ressourcenbedarf der Vernetzung. Das Beispiel der Vernetzung durch System soll diese Aussagen illustrieren.

Herrmann (1994, S. 28) entwirft einen umweltgeschichtlichen Ansatz, der die Integration von Natur- und Kulturwissenschaften auf der Grundlage der Systemtheorie leisten möchte. Das System bietet eine Formelsprache, um die komplexen Wirkungen innerhalb und zwischen Kultur und Natur differenziert abzubilden. Die Präzision und teilweise Rechenbarkeit von Systemen scheinen die Probleme der Interdisziplinarität in Harmonie aufzulösen. Dennoch stecken im Systemansatz implizit alle interdisziplinären Konflikte und können nur durch begleitendes Verbundmanagement gelöst werden.

Erstens erreicht der Systemansatz erst die wissenschaftliche Qualität einer Disziplin, wenn er sich in eine materielle Theorie rückbinden lässt. Ohne diese theoretische Fundierung sind Systeme formale Theorien mit nicht bestimmbarer Aussagenkraft über die ,reale“ Welt in unserem Fall die Umweltgeschichte. Herrmann löst das Problem, indem er auf Begriffe der Ökologie als Naturwissenschaft Bezug nimmt und hofft, dass die Kulturwissenschaften diese übernehmen können. Solches wird aber nicht der Fall sein, weil für die Kulturwissenschaften in den Begriffen geronnene Theorien und Weltbilder stecken, die in der naturwissenschaftlichen Definition verschwinden. Das Problem löst sich nicht harmonisch im z.B. gemeinsamen Zeitbegriff sondern der Konflikt wäre anzuerkennen und klar zugunsten einer Basisdisziplin zu entscheiden. Der Systemansatz ist in Verbindung mit einer materiellen Theorie sehr leistungsfähig, aber erspart nicht, die unvermeidlichen Konflikte zwischen den Disziplinen durch durchsetzungsfähige Entscheidungen zu klären.

Zweitens erzeugt die Aufarbeitung mehrerer Disziplinen durch den Systemansatz die Notwendigkeit, einzelne Disziplinen auszuwählen und andere aus der Forschung auszuschließen. Dies könnte rein wissenschaftlich am erwarteten Beitrag zum Systemergebnis entschieden werden. Dennoch werden die ausgeschlossenen Disziplinen die Argumentation nicht nachvollziehen und akzeptieren, weil aus deren Sicht Erkenntnis nur an den eigenen disziplinären Kriterien gemessen wird. Da der fehlende Beitrag zum System als solches disziplinär nicht negativ aufscheint, lässt sich der Ausschluss nicht disziplinär begründen und muss vom System gegen die Zustimmung der Einzeldisziplin entschieden werden. Die einfachste Lösung für die Praxis besteht darin, dem System alle Verfügungsmacht 
Umweltgeschichte durch starke Disziplinen

über die finanziellen Zuwendungen zu geben. Es kann dann Finanzmittel einfach von jenen Disziplinen abziehen, die es nicht braucht. Ein Konsensmodell würde scheitern (vgl. Krott 1994, S. 200).

Drittens stehen die Ressourcen, mit denen disziplinär geforscht wird, mit denen, die in die Vernetzung fließen in Konkurrenz. Die Hoffnung, disziplinär finanzierte Projekte würden aus Eigeninteresse Zeit und Personal für Vernetzung aufbringen, steht häufig am Anfang der Verbundprojekte. Sie erweist sich aber rasch als trügerisch. Je mehr sich das disziplinäre Arbeiten warm läuft, umso weniger Aufmerksamkeit und Zeit steht für Vernetzung zur Verfügung. Diesem Trend, der interdisziplinäre Versuche häufig zum Scheitern bringt, lässt sich mit der Festlegung von Ressourcen, z.B. 20 \% des Projekthaushaltes, gegensteuern, die nur in Vernetzung investiert werden dürften. Der Erfolg hängt auch hier von einer explizit ausgearbeiteten Vernetzungsstrategie ab, die für auftretende disziplinäre Konflikte Managementlösungen bereit hält und die Ressourcen realistisch abschätzt.

\section{Praxisrelevanz nach Praxiskriterien}

Die Bedeutung der Umweltgeschichte für die (politische) Lösung der aktuellen Umweltprobleme dient häufig zur Rechtfertigung dieses neuen Forschungsansatzes in der Geschichtswissenschaft. Brüggemeier (2003, S. 4) diskutiert den Beitrag der Umweltgeschichte zur „Gegenwartsdiagnose“ ausführlich und hofft, wissenschaftlich aufgearbeitete historische Erfahrungen würden Hilfestellung für die umweltpolitische Debatte anbieten. Solche Wirkungen könnten in der Tat den Einsatz von Ressourcen in umwelthistorische Forschung zusätzlich zum rein wissenschaftlichen Ziel Erkenntnisgewinn rechtfertigen. Starke Praxiswirkung wird von umfassenden „,interdisziplinären“ Analysen erwartet, die viele Aspekte der Praxisprobleme von Ökologie über Ökonomie bis zur Politik historisch behandeln. Der umfassende, historisch erweiterte Blick enthält Potential für eine rational verbesserte Gegenwartsdiagnose.

So richtig diese Feststellung ist, so wenig wird sie den Anforderungen der aktuellen Umweltpolitik gerecht. Denn den umweltpolitischen Akteuren geht es um die Durchsetzung ihrer spezifischen Interessen und nicht um umfassende Problemdiagnose an sich (vgl. Krott u. Böcher 2008). Für die Untermauerung einer Interessenposition oder die Unterstützung einer aus Gründen der Interessen bevorzugten Problemlösung eignet sich eine wissenschaftliche Argumentation häufig besser, die nur einen beschränkten Ausschnitt behandelt. Umweltinteressen begrüßen eine Fokussierung auf den Gefahrenaspekt, Industrieinteressen begründen den Lösungsbeitrag von Technologie gerne auch mit historischen Beispielen. Die Forstgeschichte ist voll von historischen Analysen der Wohltaten der Einführung neuer Technologien, wie z.B. der Säge. Ihre große Akzeptanz in der Praxis beruht auf der legitimationsstiftenden Wirkung der eingeschränkten Perspektive für den 
Forstsektor. Nachhaltige Forstwirtschaft wird auf Lösungsbeiträge historisch untersucht, störende Umweltaspekte, die das Idealbild trüben könnten, werden dabei von der Forstgeschichte gar nicht thematisiert.

Auch die Umweltgeschichte wird eine erfolgreiche Praxiskarriere mit eingeschränkter Perspektive eher erreichen als mit umfassender Analyse. Als Warnerin vor Umweltgefahren mit historischen Beispielen von katastrophalen Auswirkungen vernachlässigten Umweltschutzes wäre Umweltgeschichte der Umweltbewegung hoch willkommen. Relativierend wirkende umfassende Befunde, die auch die große Stabilität von Ökosystemen historisch zeigen, sind dagegen in der politischen Praxis bei den Schutzinteressen weniger beliebt und werden einfach ignoriert. Die politische Selektion von wissenschaftlicher Information bestimmt den Praxistransfer. Dafür ist eine eingeschränkte umwelthistorische Perspektive oft besser geeignet als die umfassende Analyse. Das häufig vorgebrachte Argument, hohe Transdisziplinarität im Sinne von großem Praxistransfer erfordere eine umfassende ,interdisziplinäre“ Perspektive, ist nicht zutreffend.

Die mangelnde Anschlussfähigkeit umfassender Perspektiven an die Handlungslogik der Praxis hat auch die stadtökologische Nachhaltigkeitsforschung erfahren müssen. Die systemaren Problemanalysen und Lösungsvorschläge passen nicht zu den eingeschränkten Perspektiven, die einzelne Fachbehörden oder Interessengruppen vertreten. Selbst bei Aufgeschlossenheit für die systemaren $\mathrm{Zu}$ sammenhänge liefern die Fachgesetze nur eingeschränkte Kompetenzen, die für systemare Lösungen nicht ausreichen. Als Strategie wird empfohlen, die systemaren wissenschaftlichen Erkenntnisse wieder in Fachperspektiven zu übersetzen. Die Feststellung, dass die Politik für umfassende Analysen keine Verwendung hat, spricht diesen nicht den hohen wissenschaftlichen Erkenntniswert ab. Sie informiert nur über die Bedingungen der Praxisrelevanz, die nicht vom Ideal der Erkenntnis sondern von den Wünschen einflussreicher politischer Akteure bestimmt werden. Hier gilt, die umfassende ,interdisziplinäre“ Perspektive der Umweltgeschichte fördert deren Praxistransfer nicht.

\section{Integration ohne Interdisziplinarität}

Abschließend soll noch auf die Option der Integration von disziplinärem Wissen durch multidisziplinäre Verfahren verwiesen werden. Beliebt ist der Einsatz von geographischen Informationssystemen, um mehrdimensionale thematische Karten zu erstellen. In einer solchen Karte lassen sich unterschiedliche disziplinäre Informationen räumlich überlagen. Das eröffnet natürlich auch die Option, zusätzlich unterschiedliche zeitliche Zustände abzubilden. Als Ergebnis entsteht eine Karte, auf der etwa Bodenerosion und sozioökonomische Dorfgemeinschaften zu unterschiedlichen historischen Zeitpunkten räumlich überlagert werden und damit im Raum irgendwie in Beziehung stehen. 
Dieses Verfahren gibt für lokale Fragestellungen wichtige Informationen und hat darüberhinaus heuristischen Wert. Die Integration bleibt jedoch in der theoretischen Basis so unbestimmt, dass sie nicht über die in den einzelnen Disziplinen vorhandene Theorie hinausreicht. In diesem Sinne ist die Integration additiv. Hier liegt der Unterschied zur interdisziplinären Vernetzung, die für eine Disziplin Innovation bewirkt und deren Theorie voranbringt.

Multidisziplinäre Integration kann auch durch formale Systemmodelle (ohne Theoriebezug) und andere Verfahren erreicht werden. Sie stoßen bei den Disziplinen auf wenig Widerstand, weil sie deren Grenzen respektieren und nur eine zusätzliche Informationsebene aufbauen. Solches wäre auch in der Umweltgeschichte insbesondere für den lokalen Bezug nützlich. Wegen des Unterschiedes zur Interdisziplinarität ist aber nicht zu erwarten, dass die additive Integration eine Vorstufe zur interdisziplinären Vernetzung darstellt. Solche Projekte weisen keine Tendenz auf, interdisziplinär zusammenzuwachsen sondern neigen dazu, additiv Daten anzuhäufen, solange Ressourcen für die Forschung zur Verfügung stehen.

\section{Schlussfolgerungen: Interdisziplinäre Optimierung um- weltgeschichtlicher Teildisziplinen}

Als allgemeines Vokabel, das der Umweltgeschichte angefügt wird, leistet „Interdisziplinarität“ inhaltlich nichts, sondern gibt der Umweltgeschichte lediglich einen modernen Anstrich, der allerdings im politischen Diskurs um Projekte durchaus hilfreich sein kann. Erst wenn „Interdisziplinarität“ mit einem konkreten Konzept verbunden wird, kann dieses einen Weg zur Optimierung der Disziplinen aufzeigen, die sich mit umwelthistorischen Entwicklungen befassen. Die Merkmale des hier entwickelten Konzeptes umwelthistorischer Disziplinen seien im Folgenden zusammengefasst:

- Interdisziplinarität ist jene besondere Phase der Zusammenarbeit unterschiedlicher Disziplinen, in der die Grenzen zwischen den Disziplinen überschritten werden und als Ergebnis eine Disziplin einzelne Erkenntnisse, Theorien oder Methoden erweitert hat.

- Interdisziplinarität in diesem Sinne kann zu umwelthistorischen Teildisziplinen führen, die innovativ eine neue theoretische oder methodische Basis gefunden haben, um bestimmte historische (kulturwissenschaftliche) Ansätze mit ausgewählten naturwissenschaftlichen Ansätzen zu verbinden. Sie fokussieren auf die Kernfrage der „Umwelt“"-,Geschichte“, Wechselwirkungen zwischen Natur und Kultur im Zeitverlauf zu analysieren. Je nach ausgewählten Disziplinen wird es unterschiedliche umweltgeschichtliche Teildisziplinen geben, die jeweils ihren spezifischen Beitrag leisten.

- Interdisziplinarität kennzeichnet die besonders innovative, aber auch konfliktreiche Entstehungsphase von neuen Einzelerkenntnissen für beteiligte 
Disziplinen oder von neuen Theorien oder Methoden, die sogar Grundlage für neue umweltgeschichtliche Teildisziplinen werden können. Es bedarf professioneller Verfahren, um Konflikte mit beteiligten Disziplinen zu regeln und Ressourcen für diese aufwendige und risikoreiche Aufgabe zu sichern. Interdisziplinarität ist kein Konzept für den Forschungsalltag der Umweltgeschichte, sondern sie macht nur in Ausnahmesituationen Sinn, wenn disziplinäre oder multidisziplinäre Zugänge zu wenig leisten oder wenn neue (leistungsfähigere) umwelthistorische Teildisziplinen geschaffen werden sollen.

- Umfassende umwelthistorische Analysen haben ihren besonderen wissenschaftlichen Wert. Dieser bedingt nicht gleichzeitig hohe Praxisrelevanz. Im Gegenteil: Einfache Erkenntnisse werden wegen ihres klaren Interessenbezuges vom politischen Diskurs bevorzugt. Aus dem Praxisbedarf lässt sich der Aufbau komplexer umwelthistorischer Disziplinen nicht begründen.

- „Ganzheitlichen Ansätze“ für die Umweltgeschichte dürfen von „Interdisziplinarität" keine Lösungen erwarten, weil Interdisziplinarität stets und dauerhaft Disziplinen voraussetzt. Multidisziplinäre Verfahren zur Integration von Erkenntnissen stehen z.B. in Form der geographischen Informationssysteme bereit. Ihre Verknüpfungsleistung bleibt jedoch in Bezug auf Theorie weit hinter dem Ergebnis einer erfolgreichen interdisziplinären Verbindung zurück. 


\section{Literatur und Quellen}

Brüggemeier, F.-J. (2003): Umweltgeschichte - Erfahrungen, Ergebnisse, Erwartungen. Archiv für Sozialgeschichte 43, S. 1-18.

Brunner, K. (1997): Virtuelle und wirkliche Welt - Umweltgeschichte als Mentalitätsgeschichte. In: Spindler, K. (Hg.) Mensch und Natur im mittelalterlichen Europa. Schriftenreihe der Akademie Friesach, Wieser Verlag. Klagenfurt.

Defila, R., Di Giulio, A. u. Scheuermann, M. (2006): Forschungsverbundmanagement. Vdf Hochschulverlag AG Zürich.

Freytag, N. (2006): Deutsche Umweltgeschichte-Umweltgeschichte in Deutschland. Historische Zeitschrift, Band 283, S. 383-407.

Herrmann, B. (1994): Umweltgeschichte als Integration von Natur- und Kulturwissenschaften. In: Bayerl, G., Fuchsloch, N. u. Meyer, T. (Hrsg.) Umweltgeschichte - Methoden, Themen, Potentiale. Waxmann Münster/New York/ München/Berlin, S. 21- 30.

Krott, M. (1994): Management vernetzter Umweltforschung. Böhlau Verlag Köln, Weimar, Wien.

Krott, M. (1996): Interdisziplinarität im Netz der Disziplinen. In: Balsinger, P., Defila, R. u. Di Guilio, A. (Hrsg.): Ökologie und Interdisziplinarität - eine Beziehung mit Zukunft? Birkhäuser Verlag, Basel, S. 87-97.

Krott, M. (2001): Interdisziplinarität in der Forstforschung. Allgemeine Forst- und Jagdzeitung, 172 Jg., Heft 2, S. 27-30.

Krott, M. (2005): Controlling für die Programmforschung. Bundesministerium für Bildung, Wissenschaft und Kultur, Wien.

Krott, M. u. Böcher, M. (2008): The acquisition of scientific knowledge by politicians. Working Paper. Göttingen.

Pfister, Ch. (2007): Umweltgeschichte. In: Di Giulio, A., Defila, R., Hammer, T. u. Bruppacher, S. (Hrsg.): Allgemeine Ökologie. Haupt Verlag Bern. S. 51-63.

Radkau, J. (2000): Natur und Macht - eine Weltgeschichte der Umwelt. Beck: München.

Winiwarter, V. u. Knoll, M. (2007): Umweltgeschichte. Eine Einführung. Böhlau Verlag Köln, Weimar, Wien. 
Interdisziplinarität in der Umweltforschung Erfahrungen aus der Praxis

Achim Daschkeit 


\begin{abstract}
The discussion about interdisciplinarity in environmental research has a long tradition and is both theoretically and practially interesting. In this article the practial experiences with interdisciplinarity in environmental research is in the foreground. After some short remarks concerning the term interdisciplinarity, the article deals mainly with successive factors of interdisciplinary environmental research and the potentials and limits of interdisciplinary integration.

Three aspects have to be pointed out: 1) Some literature asserts that every interdisciplinary project is specific so that general conclusions could not be drawn. 2) Crucial factor of interdisciplinarity is the constitution of the research object. 3) Modern interdisciplinary projects must be well organised (project planning, communication, interal / external networks).
\end{abstract}

\title{
Einleitung
}

Die Umweltforschung hat sich in den vergangenen Jahrzehnten aus zwei Gründen rasant entwickelt: Erstens gab es infolge der so genannten Umweltbewegung seit den 1960er Jahren einen zunehmenden Bedarf an Kenntnissen über das Funktionieren von Ökosystemen. Dieses wachsende Erkenntnisinteresse ist nahe liegend, bedürfen Bewertungen über den Zustand der Umwelt detaillierter Kenntnis des Gegenstandes. Daher ist es zu einem quantitativen Wachstum der Umweltforschung gekommen. Zweitens hat sich parallel zur Ausweitung der Umweltforschung eine immer noch zunehmende Binnendifferenzierung dieses Forschungsund Wissenschaftsbereiches ergeben. Indiz hierfür ist beispielsweise das Entstehen von so genannten Bindestrich-Disziplinen, zum Beispiel Geo-Botanik, PaläoKlimatologie usw.

Das quantitative Wachstum der Umweltforschung sowie deren Binnendifferenzierung führten sehr schnell zu der Frage: Wie lassen sich die vielen Detailkenntnisse zu einem Gesamtbild der Funktionsweise von Ökosystemen zusammenfügen? Hinzu kommt, dass seit den 1980er, spätestens in den 1990er Jahren der Gegenstand „Umwelt“ nicht nur auf die natürliche Umwelt (Ökosysteme) bezogen, sondern ausgeweitet wurde: Der Mensch bzw. die Gesellschaft insgesamt sind ebenfalls in das Blickfeld der Umweltforschung gelangt, und die Umweltforschung hat damit ihren Gegenstandsbereich erweitert. Ablesbar ist dies unter anderem am Entstehen der Disziplinen Umweltsoziologie und Umweltpsychologie. Von der Sache her geht es dabei um die Folgen menschlicher Aktivitäten (Wohnen, Produzieren, Mobilität etc.) für die Umwelt und umgekehrt um die Folgen einer veränderten Umwelt auf den Menschen bzw. die Gesellschaft. Unmittelbar einsichtig ist, dass vertiefte Kenntnisse über dieses Wechselverhältnis 
von Gesellschaft und Umwelt nur in fachübergreifender Kooperation, also mit interdisziplinären und integrativen Ansätzen zu erreichen ist.

Aus diesem Grund ist es zwar ein ,altes“ - und für manche langweiliges Thema, sich mit Interdisziplinarität in der Umweltforschung zu beschäftigen; ich halte es aber nach wie vor aus zwei Perspektiven für lohnend: Zum einen in theoretischer Hinsicht (vgl. Becker u. Jahn 2006), zum anderen aus praktischen Erwägungen, wobei letztere im Fokus des vorliegenden Beitrages stehen. Zielsetzung ist es dabei, fördernde und hemmende Faktoren interdisziplinärer Integration aufzuzeigen.

\section{Begriffe}

Zunächst sind einige Merkmale der Begriffe Interdiszplinarität, Transdisziplinarität sowie Umweltforschung zu beschreiben und zu diskutieren. Es geht nicht um eine umfassende Darstellung der Inhalte, die mit diesen Begriffen verbunden sind, sondern um die Charakterisierung der Begriffe, soweit das für deren weitere Verwendung von Bedeutung ist.

Interdisziplinarität wird hier als fach- bzw. disziplinübergreifende Kooperation in Bezug auf einen definierten Gegenstandsbereich verstanden. Art und Umfang dieser Kooperation können (stark) variieren; in der Umweltforschung ist es nahe liegend, aber nicht zwingend, dass diese Kooperation aus Natur-/ Ingenieurwissenschaften einerseits sowie Sozial- und Geisteswissenschaften andererseits besteht. Ziel interdisziplinärer Kooperation ist die Integration verschiedener Sachverhalte. Entscheidend für die folgenden Ausführungen ist, dass Interdisziplinarität wissenschaftsintern ist, der Anstoß oder die Motivation für interdisziplinäre Forschung können jedoch aus der politischen oder allgemein gesellschaftlichen Debatte stammen (vgl. Daschkeit u. Loibl 2007).

Demgegenüber ist transdisziplinäre Umweltforschung sowohl durch wissenschaftsinterne als auch durch wissenschaftsexterne Elemente charakterisiert und setzt oftmals auf interdisziplinärer Forschung auf; zentral ist, dass die wissenschaftsexternen Bezüge im Mittelpunkt des Forschungsprozesses stehen. Von der Konstitution des jeweiligen Forschungsgegenstandes bis hin zur Umsetzung und Vermittlung von Forschungsergebnissen spielen gesellschaftliche Akteure eine zentrale Rolle. Das bedeutet, dass transdisziplinäre Forschung eng an lebensweltliche Prozesse gekoppelt ist und in der Pflicht steht, wissenschaftliche Ergebnisse und gesellschaftliche Schlussfolgerungen zum Beispiel in Form von Empfehlungen und Handreichungen für politische Prozesse trennscharf zu differenzieren, um nicht deskriptiv-analytische und normative Elemente miteinander zu vermischen (vgl. Jahn 2005).

Umweltforschung wird hier in einem sehr breiten Verständnis verwendet: sie beschäftigt sich mit der Dynamik des Mensch-Umwelt-Verhältnisses, also den bereits erwähnten Rückkopplungen zwischen Gesellschaft und Umwelt. In diesem 
Interdisziplinarität in der Umweltforschung

Verständnis können so ziemlich alle natürlichen und sozialen Prozesse auf der Erde Gegenstand der Umweltforschung sein - das beste Beispiel hierfür ist die „Global Change“-Forschung; aber eine Einschränkung des Gegenstandsbereiches der Umweltforschung würde für den Zweck dieser Darstellung keinen Sinn machen. Für diesen Beitrag viel wichtiger ist der Umstand, dass sich die „klassische“ Umweltforschung in den letzten 10 Jahren zunehmend zu einer so genannten „Nachhaltigkeitswissenschaft“ („Sustainability Science“) gewandelt hat bzw. sich noch wandelt. Damit kommt zum Ausdruck, dass es einen Trend von interdisziplinärer zu transdisziplinärer Umweltforschung gibt, denn die Ausrichtung der Umweltforschung am Nachhaltigkeitskonzept ist nichts anderes als eine normative Aufladung der Umweltforschung. Dies hat sicherlich den Vorzug, dass die gesellschaftliche Bedeutung und „Nützlichkeit“ von Wissenschaft direkter sichtbar wird; es birgt allerdings auch die Gefahr, dass Wissenschaft zunehmend - im wahrsten Sinne des Wortes - in den Dienst politischer Interessen gestellt wird und ihre Orientierung an wissenschaftlichen Gütekriterien einbüßt (vgl. Beck 1974).

\section{Erfolgsbedingungen interdisziplinärer Umweltforschung}

Im Folgenden werden einige ausgewählte Aspekte interdisziplinärer Umweltforschung angesprochen, die aus meiner Sicht zentral sind und in der Diskussion über Interdisziplinarität immer wieder aufscheinen. So finden sich in der Literatur (beispielsweise Lange 2003) Thesen, dass

1) Interdisziplinarität nur individuell ,, beim einzelnen Forscher" stattfindet. Damit ist impliziert, dass die Verknüpfung und Integration von Wissensbeständen erst - und nur - im Zuge individuellen Lernens und zunehmenden Wissens erfolgen kann. Man kann diese These natürlich vertreten, die praktische Realisierung dürfte angesichts eines immer größer werdenden Wissensbestandes zumindest schwieriger werden;

2) Interdisziplinarität nur in projektförmig organisierter Gruppenarbeit stattfindet. Diese These schließt die erstgenannte zwar nicht aus, sie weist aber darauf hin, dass diese Form von Interdisziplinarität faktisch deutlich häufiger vorkommen dürfte.

Beide Thesen wurden meines Wissens nie empirisch geprüft, dennoch soll davon ausgegangen werden, dass Interdisziplinarität in der Umweltforschung überwiegend in Form von projektförmig organisierter Teamarbeit stattfindet.

Hinsichtlich der Erfolgsbewertung interdisziplinärer Umweltforschung finden sich in der Literatur (Bergmann u. Schramm 2008) Beschreibungen über gelungene interdisziplinäre Projekte, die zumeist von Personen stammen, die selbst an interdisziplinärer Forschung beteiligt waren. Demgegenüber gibt es nur sehr wenige empirische Studien, die sich aus neutraler Perspektive mit Erfolgsbedingungen interdisziplinärer Projekte beschäftigen (vgl. z.B. Röbbecke et al. 2004). Man müsste sich an dieser Stelle vertieft der Problematik der Bewertung interdisziplinä- 
rer Projekte und entsprechender Indikatoren zuwenden, denn erst ein hinreichend ausgearbeiteter Bewertungsmaßstab würde einen Vergleich über verschiedene Projekte hinweg überhaupt ermöglichen. Dieser Aspekt bleibt im Folgenden ausgeklammert, weil diese Thematik den Rahmen sprengen würde und es dazu einige hilfreiche Literatur gibt (vgl. Bergmann u. Schramm 2008; Daschkeit 2006; Daschkeit 2007). An dieser Stelle reicht der Hinweis, dass gemeinhin zwischen summativer und formativer Evaluation unterschieden wird. Summative Evaluation fokussiert dabei auf Forschungsergebnisse (z. B. Publikationen), formative Evaluation fokussiert hingegen auf den Forschungsprozess selbst.

Die mir bekannten empirischen Untersuchungen zu den Erfolgsbedingungen interdisziplinärer Umweltforschung stellen oftmals die nachfolgend genannten Aspekte in den Mittelpunkt:

- Zum einen wird hervorgehoben, dass eine Generalisierung der Erfolgsbedingungen kaum bzw. gar nicht möglich ist. Jedes interdisziplinäre Projekt ist so spezifisch angelegt und ausgerichtet, dass lediglich die Betrachtung von Einzelfällen möglich ist. Für eine generalisierende Aussage müsste die Vergleichbarkeit der Projekte gegeben sein, die faktisch nicht vorhanden ist.

- Zum anderen wird immer wieder betont, dass die gemeinsame Konstituierung des Forschungsgegenstandes offenbar von entscheidender Bedeutung für das Gelingen interdisziplinärer Projekte ist. Bei der Festlegung des Gegenstandes werden nämlich nicht nur verschiedene Sichten auf ein Problem nebeneinander gestellt, sondern es muss vor Beginn des Forschungsprozesses klar festgelegt werden, wie verschiedene Perspektiven auf einen Gegenstand zusammengeführt werden. Das Problem der interdisziplinären Konstitution des gemeinsamen Gegenstands ist damit ein Problem der Integration von Wissensbeständen (vgl. Becker u. Jahn 2006; Balsiger 2005).

- Letztlich werden die organisatorischen Voraussetzungen interdisziplinärer Projekte in den Mittelpunkt gestellt. Unter anderem muss die projektinterne Kommunikation geregelt und unterstützt werden, es muss eine Projektplanung mit Aufbau- und Ablauforganisation geben, ein Glossar zur begrifflichen Verständigung sollte gepflegt werden und regelmäßige Workshops müssen den Austausch und die Vernetzung fördern. Man kann hier davon sprechen, dass „,modernes Projektmanagement“ in der Umweltforschung angekommen ist.

Als Schlussfolgerungen aus dieser kurzen und keineswegs erschöpfenden Analyse der Erfolgsbedingungen interdisziplinärer Umweltforschung ist festzuhalten:

- Es gibt nur relativ wenige empirische Studien zu interdisziplinären Projekten der Umweltforschung, so dass eine breit gefächerte Diskussion bzgl. der Erfolgsbedingungen kaum stattfindet und entsprechend generalisierende Aussagen schlecht möglich sind. Das kann ein Grund dafür sein, dass die Individualität interdisziplinärer Projekte betont wird. 
- Sowohl praktische Elemente als auch konzeptionell-theoretische Elemente sind offensichtlich für das Gelingen von Interdisziplinarität maßgeblich. Die Gegenstandskonstitution ist im Zusammenhang mit den Möglichkeiten der Integration von Wissensbeständen in interdisziplinären Projekten zentral. Deswegen soll dieser Aspekt nachstehend etwas ausführlicher behandelt werden.

\section{Elemente interdisziplinärer Integration}

Es wird in diesem Beitrag argumentiert, dass „Integration“ zentrale Voraussetzung für das Gelingen von Interdisziplinarität ist. Unter „Integration“ wird hier die Verwendung von fachübergreifenden Theorien, Konzepten und Methoden verstanden, wobei es ausgewählte Elemente zu diskutieren gibt.

- Die Ausrichtung an und die Verwendung von fachübergreifenden Theorien zur Mensch-Umwelt-Dynamik spielt nach meiner Einschätzung als Instrument der Integration in der interdisziplinären Umweltforschung derzeit eine eher marginale Rolle. In den letzten Jahren entwickelte sich die Umweltforschung in Richtung „Nachhaltigkeitswissenschaft", die problemorientiert ausgerichtet ist und eher unter dem Etikett „Transdisziplinarität“ geführt wird. In erster Linie geht es hier um die Bearbeitung gesellschaftlich als relevant definierter Probleme; Interdisziplinarität wird als selbstverständlicher Teil von Transdisziplinarität aufgefasst. Jenseits dessen gibt es Ansätze, die Theorie in den Mittelpunkt interdisziplinärer Integration stellen oder Theorie zumindest stark gewichten, so bspw. der Ansatz der Sozialen Ökologie (vgl. Becker u. Jahn 2006). Insgesamt führt die Orientierung interdisziplinärer Umweltforschung an einer Nachhaltigkeitswissenschaft allerdings eher dazu, Theorieelemente zu vernachlässigen.

- Eine weitere Orientierung interdisziplinärer Integration ist an der Beantwortung von Ausgangsfragestellungen ausgerichtet: das mag sich im ersten Moment „,banal“ anhören; es gibt aber positive Beispiele, wo Ausgangsfragestellungen große Verbundprojekte in der Umweltforschung strukturiert haben; so zum Beispiel die Ökosystemforschung Wattenmeer an der schleswig-holsteinischen Westküste in den 1990er Jahren, indem die Novellierung des Nationalparkgesetzes Bezugspunkt vielfältiger disziplinärer und interdisziplinärer Arbeiten war. Aus der Perspektive der Ressortforschung z. B. des Umweltbundesamtes ist diese Herangehensweise bekannt und übliche Praxis. In der Ressortforschung standen ,schon immer" praktische und politisch relevante Fragestellungen im Vordergrund.

Gleichzeitig wird die Ansicht vertreten, dass ein solches Vorgehen nicht als klassische Forschung bezeichnet werden kann, weil die Aufdeckung von Gesetzmäßigkeiten in der Umwelt oder der Gesellschaft nicht im Mittelpunkt steht. Es wird oftmals unterschieden zwischen (i) zweckfreier Er- 
kenntnisgewinnung und (ii) spezifischer Erkenntnisgenerierung, welche zur Erfüllung staatlicher Aufgaben dient. Man könnte diese Unterteilung auch als Angebots- vs. Nachfrageorientierung bezeichnen.

- Eine weitere Integrationsmöglichkeit wird in der Verwendung von Modellen gesehen. Ich verwende hier einen weit gefassten Modellbegriff, der von Konzeptmodellen bis hin zu mathematisch ausformulierten numerischen Modellen reicht. Ein weithin bekanntes Beispiel sind die Modelle der Klimaforschung. Weitere Beispiele sind die Struktur einer umwelthistorischen Untersuchungseinheit (vgl. Winiwarter u. Knoll 2007, S. 76) und der so genannten DPSIR-Ansatz (Driving Forces - Pressures - State - Impacts Responses). In vielen Modellen werden naturwissenschaftliche Sachverhalte sehr umfassend abgebildet, die Integration ökonomischer Sachverhalte ist ebenfalls weit vorangeschritten, die Integration gesellschaftlicher Aspekte ist demgegenüber noch kaum realisiert. In meinen Augen ist Modellierung die konsequente Fortsetzung des Projektmanagements bis in die inhaltliche Arbeit hinein. Es kommt zu einer Input-Output-Schnittstellen-

Orientierung. Ob dies positiv oder negativ zu beurteilen ist, lässt sich nicht eindeutig beantworten. Aus sozial- und geisteswissenschaftlicher Perspektive wird man vermutlich eher etwas skeptisch sein, in den Natur- und Ingenieurwissenschaften sowie der integrierten Klimaforschung ist diese Orientierung gang und gäbe. Röbbecke et al. (2004) zeigen, dass Modelle sowohl in kognitiver als auch in sozialer Hinsicht sehr hilfreiche Instrumente zur Wissensintegration sind. Schuchardt et al. (2007, S. 182) haben bei Projekten der interdisziplinären Klimaforschung danach gefragt, ob und wie die Aufgabenwahrnehmung durch zentrale Organisationseinheiten erfolgt. Hier zeigt sich, dass die inhaltliche Steuerung des interdisziplinären Forschungsprozesses von $21 \%$ der Befragten als Aufgabe der zentralen Organisation angesehen wird, lediglich 3\% der Befragten antworteten, dass dies durch die Anwendung integrativer Werkzeuge - z. B. Modelle - erfolgt.

- Ganz ähnlich wie Modelle sind so genannte Entscheidungsunterstützungssysteme (EUS; DSS = decision support system) zu sehen: Sie sind zumeist nichts anderes als Modelle, die im Prinzip für den entscheidungsbezogenen Einsatz in Politik, Verwaltung, Wirtschaft konzipiert sind. Deshalb gilt das eben Ausgeführte in analoger Form, nur dass EUS mit einer handhabbaren Benutzeroberfläche versehen sind bzw. versehen sein sollten. Da sie als Integrationsinstrument für interdisziplinäre Umweltforschung versuchen, darüber hinaus Entscheidungsträger (stakeholder) zu integrieren, spielen sie in der Diskussion um Transdisziplinarität eine wichtige Rolle.

- Szenarien als eine weitere Möglichkeit zu Integration lassen sich ähnlich wie Modelle in verschiedener Weise definieren. Sie sind oftmals Teil von Modellen und EUS und von daher ein durchaus nützliches Integrationsinstrument. Szenarien werden bei der Kopplung mit Modellen und EUS quantifi- 
ziert, haben aber außerdem eine „,heuristische Funktion“, wenn sie beispielsweise als „story lines“ bei IPCC (Intergovernmental Panel on Climate Change) oder beim MEA (Millenium Ecosystem Assessment) formuliert werden. Beim Erstellen von und Arbeiten mit Szenarien lernt man Möglichkeiten und Grenzen der Projektion von natürlichen und gesellschaftlichen Entwicklungen und ist gezwungen, Wechselwirkungen in der MenschUmwelt-Dynamik zu konkretisieren und zu operationalisieren. Wenn man also Szenarien nicht nur quantitativ sondern auch qualitativ denkt, können sie aus meiner Sicht ein hilfreiches Integrationsinstrument sein.

- Geographische Informationssysteme (GIS) werden in der interdisziplinären Umweltforschung in zunehmendem Maße als Werkzeug eingesetzt und sind oft ein Teil von Modellen oder EUS. Auch für die Erstellung von und die Arbeit mit Szenarien werden GIS oftmals als räumliche Datenbank und Visualisierungsmöglichkeit von verschiedenartigen Sachverhalten verwendet. Ausgangspunkt ist das Layerprinzip, d.h. die Darstellung einzelner Sachverhalte in Kartenform mit der Möglichkeit, einzelne Karten (Informationsschichten, synonym: Layer) zu verknüpfen. Diese Verknüpfung benötigt aber eine „Hintergrundtheorie“ oder zumindest Hypothesen darüber, wie die verschiedenartigen Informationsschichten zusammenhängen. Die Integration erfolgt dann entweder über Verschneidungsalgorithmen oder mathematische Beziehungen. GIS sind m. E. ein hilfreiches Integrationsinstrument in der interdisziplinären Umweltforschung. Sie sind zunächst nicht ganz so voraussetzungsreich wie Modelle und mittlerweile in Umweltforschungsprojekten Standard, vor allem wenn flächenhafte Aussagen gemacht werden sollen.

- Die Ausführungen zu GIS im vorigen Absatz legen auf den ersten Blick nahe, dass hiermit gleichsam die Möglichkeit der Integration über den Begriff „Raum“ beschrieben ist, sind doch GIS nichts anderes als die Visualisierung räumlicher Informationen. Auf den zweiten Blick müssen allerdings zwei verschiedenartige Zugänge unterschieden werden: Zum einen ein theorieorientierter Strang, der u. a. in der Geographie, in der Soziologie sowie den Regional- und Planungswissenschaften geführt wird. Hier gibt es meiner Kenntnis nach sehr unterschiedliche Positionen, die von naturalistischen über konstruktivistische bis hin zu pragmatischen Raumkonzepten reichen. Es gilt dann in gewissem Umfang das, was hinsichtlich des Theoriebezugs ausgeführt wurde. Ein zweiter Strang bezieht sich auf RaumVergleiche im Sinne von Fallstudien. Diese räumlich vergleichenden Betrachtungen sind aber zumeist nichts anderes als Einzelfallbetrachtungen, weil die Randbedingungen der räumlich vergleichenden Betrachtung eben nicht wie im klassischen Experiment systematisch variiert werden können. Somit fehlt eine Art „Theorie bzw. Methodologie des Vergleichs“. „Raum“ als Theorie oder Konzept hat insgesamt vielleicht noch gar nicht das Po- 
tenzial als Instrument zur Integration in der interdisziplinären Umweltforschung ausgeschöpft.

\section{Fazit}

In den letzten 10 bis 15 Jahren sind zwar nicht sehr viele, aber dennoch einige Untersuchungen zur interdisziplinären Praxis in der Umweltforschung durchgeführt worden. Zahlreiche dieser Untersuchungen sind sehr aufschlussreich und haben unter anderem zur Entwicklung von konkreten Hilfestellungen zur Planung und Durchführung interdisziplinärer Projekte geführt (vgl. Daschkeit 2006; Daschkeit 2007). Parallel hat sich die Diskussion zu Interdisziplinarität mehr und mehr in eine Diskussion über Transdisziplinarität gewandelt. Vornehmlich lag das daran, dass Problemorientierung und Anwendung in der Herausbildung einer Nachhaltigkeitswissenschaft an Bedeutung gewonnen haben und dass Interdisziplinarität in der Umweltforschung ,stillschweigend“ vorausgesetzt wird. Gleichzeitig hat diese Entwicklung dazu beigetragen, sich über die Beurteilung von Interund Transdisziplinarität mehr Gedanken zu machen: Wie ist inter- und transdisziplinäre Forschung zu bewerten? Wo verläuft die Grenzlinie zwischen Wissenschaft bzw. Forschung und anderen gesellschaftlichen Teilsystemen? Welche Indikatoren sind für die Bewertung inter- und transdisziplinärer Forschung angemessen?

Systematisch und empirisch angelegte Analysen der Praxis interdisziplinärer Umweltforschung, mit engem Bezug auf den Aspekt „Integration“ sind sehr selten. Deswegen sind generalisierende Aussagen zu den Erfolgsbedingungen von Interdisziplinarität und zur Funktionsweise von Instrumenten zur Integration verschiedener Wissensbestände m. E. auch sehr schwer abzuleiten. Kurzum, es gibt kein Patentrezept, wie „gute“ interdisziplinäre Praxis aussieht. In der allgemeinen Wissenschaftsforschung - nicht nur in Bezug auf Umweltforschung wird die zentrale soziale und kognitive Funktion von Disziplinen als Basis für Interdisziplinarität hervorgehoben. Möglicherweise ist dies einerseits eine ernüchternde Erkenntnis aus vielen Jahren Diskussion über Interdisziplinarität und der nach wie vor enormen Bedeutung von Wissenschaftsdisziplinen, andererseits ist der Ruf nach fachübergreifender, interdisziplinärer Forschung angesichts komplexer Problemlagen wie dem Klimawandel unüberhörbar. 


\section{Literatur und Quellen}

Balsiger, P. W. (2005): Transdisziplinarität. München.

Beck, U. (1974): Objektivität und Normativität. Die Theorie-Praxis-Debatte in der modernen amerikanischen und deutschen Soziologie. Wiesbaden.

Becker, E. u. Jahn, T. (Hrsg.) (2006): Soziale Ökologie: Grundzüge einer Wissenschaft von den gesellschaftlichen Naturverhältnissen. Frankfurt am Main, New York.

Bergmann, M. u. Schramm, E. (Hrsg.) (2008): Transdisziplinäre Forschung: Integrative Forschungsprozesse verstehen und bewerten. Frankfurt am Main, New York.

Daschkeit, A. (2006): Von der naturwissenschaftlichen Umweltforschung zur Nachhaltigkeitswissenschaft? In: GAIA, Bd. 15/1, S. 37-43.

Daschkeit, A. (2007): Zur Beurteilung transdisziplinärer Forschung. Hinweise auf Bücher zu Transdisziplinarität. In: GAIA, Bd. 16/1, S. 58-65.

Daschkeit, A. u. Loibl, C. (2007): Projektspezifische Evaluation humanökologischer Nachhaltigkeitsforschung - Entwurf eines Bewertungsschemas. In: Stoll-Kleemann, S. u. Pohl, C. (Hrsg.): Evaluation inter- und transdisziplinärer Forschung. Humanökologie und Nachhaltigkeitsforschung auf dem Prüfstand. Edition Humanökologie, Band 5. München. S. 157-171.

Jahn, T. (2005): Soziale Ökologie, kognitive Integration und Transdisziplinarität. In: Technikfolgenabschätzung Theorie und Praxis, Bd. 14/2, S. 32-38.

Lange, H. (2003): Interdisziplinarität und Transdisziplinarität. Eine „,Wissenschaft neuen Typs“ oder „vergebliche Liebesmüh“? In: Linne, G. u. Schwarz, M. (Hrsg.): Handbuch Nachhaltige Entwicklung. Wie ist nachhaltiges Wirtschaften machbar? Opladen. S. 563-574.

Röbbecke, M., Simon, D., Lengwiler, M. u. Kraetsch, C. (2004): Inter-

Disziplinieren. Erfolgsbedingungen von Forschungskooperationen. Berlin.

Schuchardt, B., Birner, R., Osthorst, W. u. Bildstein, T. (2007): Empirische Erfahrungen mit der Organisation interdisziplinärer Umweltforschung. In: Zeitschrift für angewandte Umweltforschung, Bd. 18/2, S. 172-186.

Winiwarter, V. u. Knoll, M. (2007): Umweltgeschichte. Stuttgart. 


\section{Eine historisch-ökologische Sicht auf Landnut- zungsgeschichte und Ökosystementwicklung}

Matthias Bürgi*

* Ich danke den beiden anonymen Gutachtern für ihre hilfreichen Kommentare und Anmerkungen zu einer früheren Version des Artikels. 


\begin{abstract}
Most ecosystems and landscapes worldwide are dominated or influenced by human impacts. Consequently, studies of patterns and processes of and within anthropogenic ecosystems and cultural landscapes have to consider human impacts and their historical development adequately. Three major objectives of historical ecology, i.e., the study of human impacts on ecosystems and landscapes over time, can be distinguished: (a) preserving cultural heritage in ecosystems and landscapes, (b) understanding historical trajectories of patterns and processes in ecosystems and landscapes, and (c) informing ecosystem and landscape management. In this paper, the application of these three major objectives of historical ecology is illustrated with a case study on litter collecting - a largely forgotten traditional forest use in Central Europe.
\end{abstract}

\title{
Einleitung
}

Wer historische Prozesse untersucht, muss anerkennen, dass diese in einem räumlichen Kontext stattfinden. Und ebenso stellt, wer ökologische Prozesse studieren will, zwangsläufig fest, dass diese eine zeitliche, ja oft sogar eine historische Dimension ausweisen. Es ist daher nicht erstaunlich, dass die wissenschaftliche Auseinandersetzung mit raum-zeitlichen Phänomenen eine lange Tradition hat. Selbst bei einer Präzisierung der Thematik auf Raum und Geschichte bleibt die Liste der entsprechend engagierten Subdisziplinen lang. Neben Historischer Geographie und Umweltgeschichte gibt es zahlreiche Studien im Bereich der Vegetationsgeschichte, Wald- und Forstgeschichte, Archäologie, Landschaftsökologie, Humanökologie, Volkskunde, etc., die sich diesem Themenfeld annehmen. Viele der erwähnten Teildisziplinen werden von eher kleinen, disparaten „Scientific Communities“ betrieben, die einander oft wenig referenzieren. Die Publikationskulturen von Volkskunde und Paläoökologie - um zwei Beispiele zu nennen - sind derart unterschiedlich, dass kaum jemand in der Lage ist, den Überblick über die wichtigsten thematischen Entwicklungen und neuesten Forschungsresultate beider Ansätze zu behalten. Es ist ein Ausdruck dieses Mangels, wenn der Vorsitzende des Arbeitskreises für Kulturlandschaftsforschung in Mitteleuropa (ARKUM e.V.) moniert, dass die Umweltgeschichte „die Befunde der in ARKUM gebündelten Disziplinen ... kaum zur Kenntnis nimmt" (Schenk 2006) - und es ist zu vermuten, dass für einige Mitglieder von ARKUM bezüglich ihrer Wahrnehmung der Befunde der Umweltgeschichte umgekehrt Ähnliches gilt.

Angesichts der Vielfalt offenbar ungenügend vernetzter Teildisziplinen mag es wenig sinnvoll erscheinen, wenn im Folgenden mit der Historischen Ökologie ein weiterer Forschungsansatz vorgestellt wird, der sich an der Disziplinengrenze von 
historischen und raumwissenschaftlichen Ansätzen lokalisiert, zumal, wie Winiwarter (2007) kürzlich absolut zurecht feststellte, im deutschen Sprachraum die Bezeichnung „Historische Ökologie“ noch kaum gebräuchlich ist. Anders verhält es sich hingegen mit dem englischen Begriff der „historical ecology“, der sich in den letzten Jahren gut etablieren konnte. Sicherlich ist allein die Tatsache, dass eine Fachrichtung im englischsprachigen Wissenschaftsraum eine wesentlich höhere Sichtbarkeit hat, als im deutschsprachigen, noch keine Garantie dafür, dass hier etwas Wesentliches vor der Türe steht. Aber genauer hinzuschauen lohnt sich allemal.

Es wird im Folgenden zu zeigen sein, was genau unter dem Begriff Historische Ökologie verstanden wird, indem die drei wichtigsten Ausrichtungen genannt werden, die die Historische Ökologie im englischen Sprachraum angenommen hat. Dabei wird deutlich werden, dass es sich dabei nicht um eine, wie von Winiwarter (2007) behauptet, ,von historisch-archäologischer Seite betriebene Form der Umweltgeschichte handelt“. Weiter soll am Beispiel einer in Vergessenheit geratenen Waldnutzungsweise, der Waldstreunutzung, illustriert werden, dass sich diese drei Ausrichtungen ergänzen und dass in ihrer Kombination eine der Stärken der Historischen Ökologie liegt. Ziel dieser Ausführungen ist somit aufzuzeigen, welchen Beitrag der historisch-ökologische Ansatz zu einer integralen Analyse der Geschichte der Landnutzung und der Ökosysteme zu leisten vermag. Selbstverständlich liegen auch in der deutschsprachig publizierten Forschung zahlreiche Studien vor, die als historisch-ökologisch ausgerichtet bezeichnet werden könnten. Einen Überblick darüber zu geben, liegt jedoch nicht in der Absicht dieses Aufsatzes.

\section{Die drei Ausrichtungen der Historischen Ökologie}

In der englischsprachigen Literatur können drei Ausrichtungen von Historischer Ökologie unterschieden werden. Zum ersten wird der Begriff von Anthropologen verwendet, die sich grundsätzlich mit Mensch-Umwelt-Interaktionen beschäftigen (z.B. Crumley 1994; Balée 1998). So hat Crumley (1994) definiert: „Historical ecology traces the ongoing dialectical relations between human acts and acts of nature, made manifest in the landscape”. Zweitens verwenden den Begriff Ökologinnen und Ökologen, die anthropogene Einflüsse auf Ökosysteme und Landschaften untersuchen (z.B. Östlund et al. 1997; Bürgi et al. 2000; Hellberg et al. 2003; Foster und Motzkin 2003). Dearing et al. (2006) forderten, dass dabei der menschliche Einfluss nicht lediglich als „Stressor“ mit einer gewissen Wirkung auf ein Ökosystem oder auf eine Landschaft betrachtet werden sollte, sondern dass die Menschen als Akteure verstanden und analysiert werden müssten. Drittens wird der Begriff auch im Kontext der Restoration Ecology und der Landschaftsplanung verwendet (z.B. Marcucci 2000; Egan und Howell 2001; Hessl 2002; Dirkx 2004). Dabei wird davon ausgegangen, dass Informationen über die Genese der Land- 
schaft, über historische Referenzzustände, über Entwicklungs-Trajektorien von Ökosystemen und Landschaften, dazu beitragen, die Qualität der Planung und des Umwelt-Managements zu verbessern. Nicht von ungefähr heißt ein bekanntes Lehrbuch von Egan und Howell (2001) „The historical ecology handbook - a restorationist's guide to reference ecosystems".

Selbstverständlich bestehen zwischen diesen drei Ausrichtungen, die man als anthropologische Grundlagen, ökologische Anwendung und planerische Umsetzung bezeichnen kann, viele Verbindungen. Dies wird schon in einer sehr frühen Verwendung des Begriffes deutlich, nämlich im Aufsatz von Norman L. Christensen mit dem Titel „Landscape history and ecological change“ (Christensen 1989). In diesem Aufsatz beschreibt Christensen die Bedeutung der Zusammenarbeit von Ökologie und Geschichte für ein vertieftes Verständnis der aktuellen ökologischen Prozesse, und er weist im Kapitel „Historical ecology as predictive endeavor" auf die große Bedeutung des Einbezugs der Beziehungen von ökologischen und historischen Entwicklungen für die Verbesserung der prognostischen Qualität ökologischer Modelle hin.

Zusammenfassend lässt sich Historische Ökologie folgendermaßen definieren: Historische Ökologie befasst sich mit der historischen Entwicklung von Ökosystemen und Landschaften unter dem Einfluss natürlicher und anthropogener Faktoren.

\section{Forschungskontext}

Bereits 1864 publizierte der amerikanische Geograph George Perkins Marsh sein Buch „Man and nature; or, physical geography as modified by human action” (Marsh 2003). Die darin enthaltene Beschreibung des menschlichen Einflusses auf sein natürliches Umfeld inspirierte die frühe Naturschutzbewegung beidseits des Atlantiks (Hall 2005). Seit den 1950er Jahren ist eine Reihe von Grundlagenwerken erschienen, die sich mit „Man's role in changing the face of the earth“ (Thomas 1956) auseinander gesetzt haben. Titel wie „The human impact on the natural environment” (Goudie 1981), „Changing the face of the earth - culture, environment, history“ (Simmons 1989) oder schliesslich explizit „People and the land through time - linking ecology and history” (Russell 1997) legen nahe, dass auch in mehrheitlich ökologisch motivierten Studien historische Informationen berücksichtigt werden müssen, wenn die Wechselwirkung gesellschaftlicher und ökosystemarer Entwicklungen diskutiert werden soll. Die Nachfrage nach derartigen integralen Studien basiert - vereinfacht gesagt - auf der Tatsache, dass anthropogen geprägte Ökosysteme und Landschaften ohne genaue Kenntnisse der relevanten menschlichen Tätigkeiten nicht adäquat untersucht und verstanden werden können (vgl. Christensen 1989; Foster et al. 2003). Diese Verbindung von Geschichte und Ökologie wurde denn auch in verschiedenen großangelegten Forschungsprogrammen erprobt, wie beispielsweise dem MAB-Program („Man and 
the biosphere“, http1), LTER („Long Term Ecological Research“, http2), or PAGES („Past Global Changes“, http3), insbesondere mit dem Fokus 5 „Past Ecosystems Processes and Human-Environment Interactions” und darin der Aktivität HITE („Human Impact on Terrestrial Ecosystems”). Der Beginn des HITEForschungsplanes liest sich denn auch wie eine weitere Definition von historical ecology: „Earth system science recognizes that knowledge of the history of environmental variability and human-environment interactions improves our understanding of the functioning of earth systems and their response to current and future impacts" (HITE 2006).

Die angestrebte Integration von Geschichte in ökologisch orientierte Untersuchungen ist konzeptionell anspruchsvoll (vgl. Nassauer 1995). Eine Herausforderung besteht darin, dass die historischen Quellen nicht mit der Absicht erstellt worden sind, später für die Beantwortung ökologischer Fragestellungen ausgewertet $\mathrm{zu}$ werden. Dementsprechend sind die Angaben oftmals nicht so präzise, umfassend und klar, wie sie sein müssten, um beispielsweise quantifiziert, statistisch ausgewertet oder in ein Geographisches Informationssystem (GIS) integriert werden zu können. Hier gilt es, der Versuchung einer Überinterpretation der Quellen $\mathrm{zu}$ widerstehen und mit einer quellenkritischen Arbeitsweise den methodischen Erfordernissen der historischen Wissenschaften zu entsprechen (vgl. Forman u. Russell 1983; Edmonds 2001). Die für die Historische Ökologie charakteristische Kombination von Informationen aus unterschiedlichen Quellentypen (vgl. Egan u. Howell 2001) kann zudem im Einzellfall helfen, die erwähnten Limitierungen einzelner Quellentypen oder konkreter Quellenbestände zu kompensieren. Zusätzlich ist aber auch die Ökologie gefordert, den Einbezug historischer Information durch die Erweiterung des Kanons akzeptierter Auswertungs- und Darstellungsweisen durch deskriptive oder sogar narrative Ansätze zu erleichtern (z.B. Allen et al. 2001; Hessburg und Agee 2003; Constanza et al. 2007).

\section{Die Waldstreunutzung als in Vergessenheit geratene Waldnutzungsform: Grundlagen, Anwendung, Umsetzung}

Bei der Darstellung der drei Ausrichtungen der Historischen Ökologie wurde vorangehend eine Einteilung in anthropologische Grundlagen, ökologische Anwendung und planerische Umsetzung vorgeschlagen, und es wurde postuliert, dass eine der Stärken dieses Forschungsansatzes in der Kombination dieser drei Ausrichtungen liege. Dies soll am Beispiel einer in Vergessenheit geratenen Landnutzungsform, der Waldstreunutzung, illustriert werden (siehe auch Bürgi u. Gimmi 2007). Der Wald als langlebiges Ökosystem ist besonders geeignet, um die Bedeutung der menschlichen Nutzungsgeschichte für das Verständnis heutiger Ökosysteme zu illustrieren (vgl. Bürgi 2003). 


\section{Grundlagen: Sicherung des Wissens}

Die meisten mitteleuropäischen Ökosysteme entstanden über Jahrhunderte unter starkem Einfluss der Landnutzung des Menschen (vgl. Küster 1995). Während einige Einflüsse flüchtig und ihre Folgen kurzlebig waren, gibt es eindrückliche Beispiele überaus persistenter Spuren. So konnten Dambrine et al. (2007) zeigen, dass in der heutigen Zusammensetzung der Krautschicht in einem zentralfranzösischen Waldgebiet die Lage der vor mindestens 1'500 Jahren aufgegebenen römischen Landwirtschaftsbetriebe sichtbar wird. Dieser Effekt kann jedoch in der Analyse der heutigen Vegetation nur berücksichtigt werden, wenn die archäologischen Informationen ausreichend vollständig sind und die mit der Studie betrauten Vegetationskundler sich der möglichen Bedeutung dieser Informationen für die Interpretation heutiger Pflanzenaufnahmen bewusst sind.

Es gibt keine Gewähr dafür, dass die für uns heute relevanten Informationen in den historischen Quellen adäquat überliefert worden sind. Dies gilt erst recht für Landnutzungsweisen, die weitgehend durch wirtschaftlich unterprivilegierte Bevölkerungskreise für die Selbstversorgung ausgeübt wurden (vgl. Abb. 1).

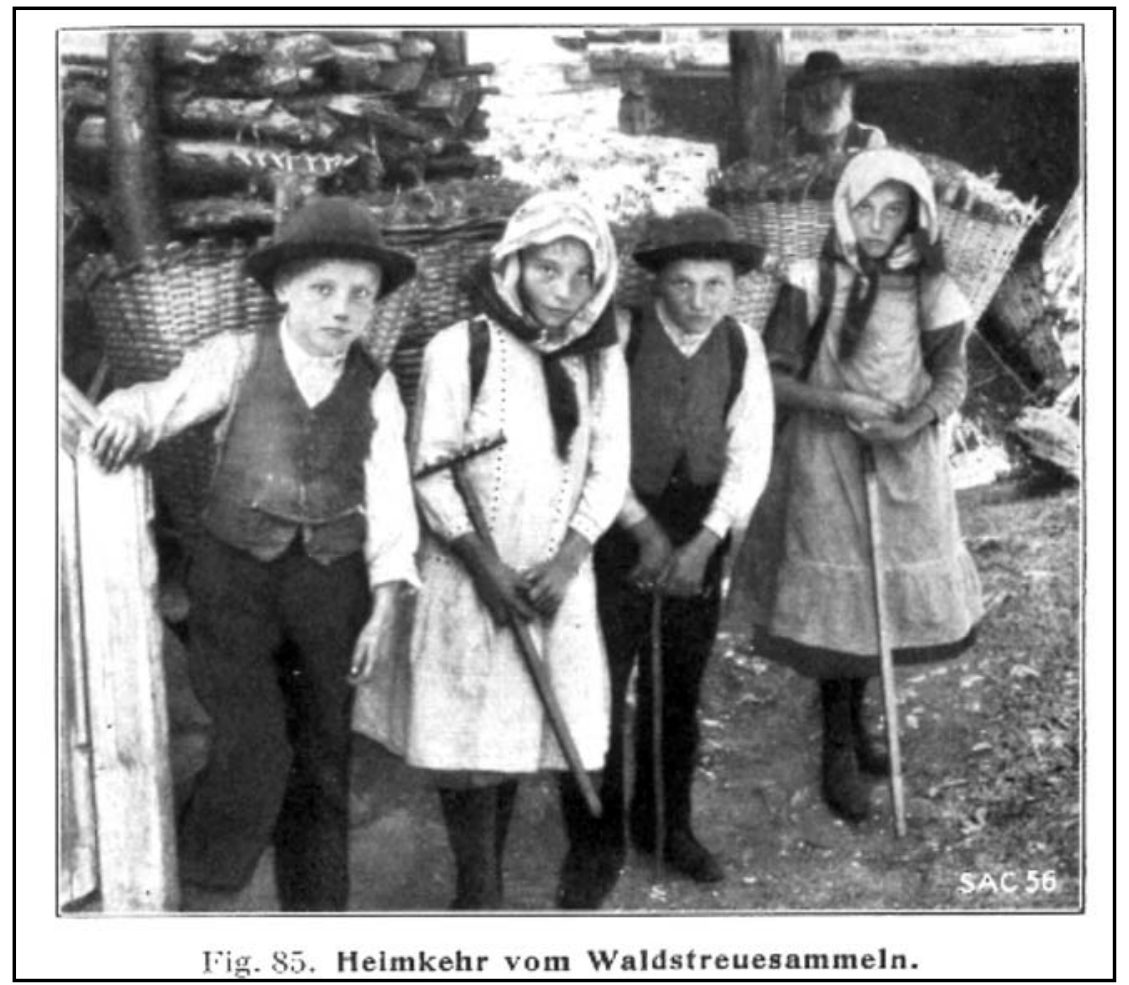

Abb. 1: Knaben und Mädchen mit gefüllten "Chris-Tschifferen“ (Eggwald, Zeneggen, Kanton VS, Schweiz. Publiziert in: Stebler 1922, S. 103) 
Nun darf jedoch die mangelhafte Überlieferung der Aktivitäten marginalisierter Bevölkerungskreise nicht mit einer marginalen Bedeutung dieser Aktivitäten für die Entwicklung der Ökosysteme verwechselt werden! So zeigt eine kürzlich erfolgte Literaturstudie über die nichtforstlichen Nutzungen der Wälder in der Schweiz von 1800 bis 1950 (vgl. Stuber u. Bürgi 2001, 2002; Bürgi u. Stuber 2003) eine Vielzahl von Nutzungsweisen mit potentiell bedeutendem Einfluss auf die Waldökosysteme. Die Informationen aus der Literatur sind jedoch oftmals nur anekdotisch und können kaum direkt für ökologische Studien fruchtbar gemacht werden.

In der Forschungsgruppe Landnutzungsgeschichte an der Eidgenössischen Forschungsanstalt für Wald, Schnee und Landschaft WSL in Birmensdorf, Schweiz, wird der Frage nachgegangen, ob nicht für ausgewählte Nutzungsweisen Zeitzeugen zu finden wären, die über die genaue Ausübung Auskunft geben könnten. Es sollte also versucht werden, die fehlenden schriftlichen Quellen durch systematisch erhobene mündliche Quellen zu ergänzen und die Überlieferungslücken mittels sogenannter Oral-History-Interviews zu stopfen.

Ein erstes Oral-History-Projekt wurde zu einem thematisch und regional eng begrenzten Thema, dem Bettlaubsammeln im St. Galler Rheintal (Roth und Bürgi 2006), durchgeführt. Die Studie zeigte, dass in weiten Teilen des Untersuchungsgebietes Bettlaub gesammelt wurde (Abbildung 2). Da meistens Buchenlaub zur Füllung der Laubsäcke genutzt wurde, reduziert sich das Verbreitungsgebiet auf die Buchenwaldstandorte. Die Studie zeigte, dass im St. Galler Rheintal im 20. Jahrhundert fast nur noch ärmere Leute Bettlaub sammelten. Das endgültige Verschwinden der Laubnutzung zog sich jedoch über eine lange Zeit hin, schliefen einzelne Familien doch noch bis in die frühen 1960er Jahre auf Laubsäcken. Die intensive Bettlaubnutzung hatte aus Sicht des Forstdienstes negative Auswirkungen auf den Wald, was als weiterer Hinweis auf die ökologische Relevanz dieser Nutzungsweise gewertet werden kann. 


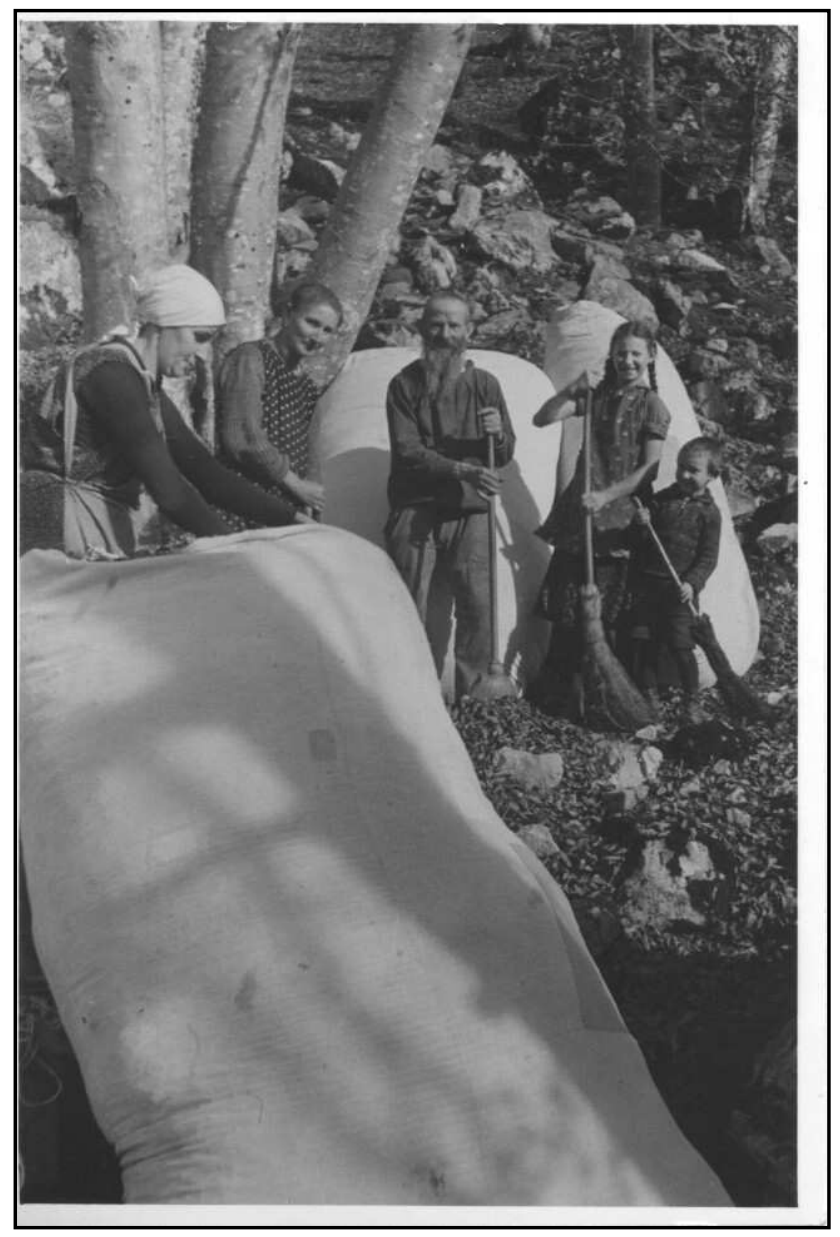

Abb. 2: „Beim Bettlauben im Gonzenwald ist die ganze Familie beschäftigt.“ (um 1940, F. MoserGossweiler, Romanshorn - Privatarchiv M. Bugg, Berschis)

In einem anderen Projekt wurde die Nutzungsgeschichte der Waldföhrenwälder im Wallis untersucht. Im Zentrum stand dabei, neben der Waldweide, wiederum die Waldstreunutzung (vgl. Abb. 3). Es zeigte sich, dass erst die Kombination von forstlichen Quellen, insbesondere den Waldnutzungsplänen, mit Oral-HistoryInterviews die Rekonstruktion einer raum-zeitlichen Nutzungsdynamik erlaubte (Gimmi u. Bürgi 2007). Dabei lag die Stärke der Zeitzeugenaussagen vor allem in der Detailfülle zur praktischen Ausübung und des sozioökonomischen Kontextes der Nutzungsweisen. 


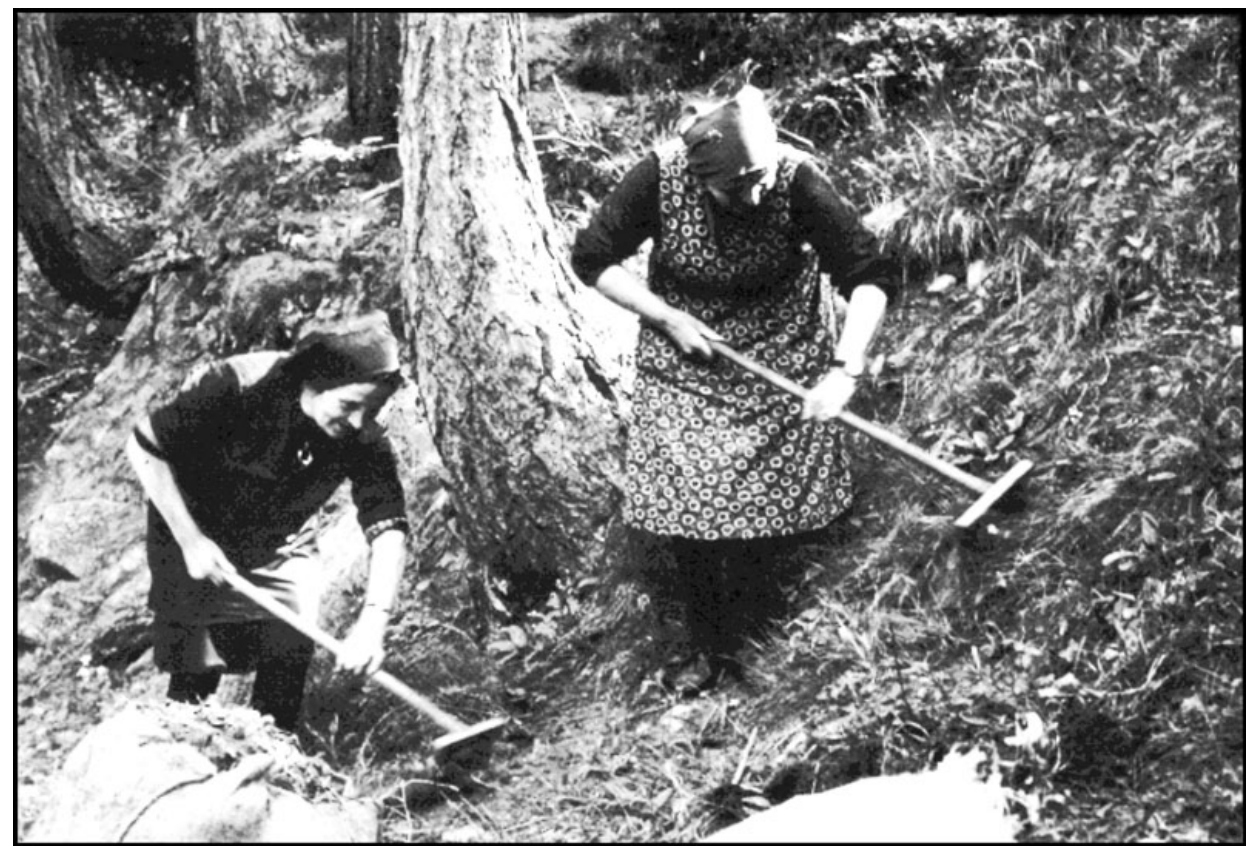

Abb. 3: Saaser Frauen kratzen Nadel- und Unkrautstreue mit Adlerrechen zusammen (um 1975, Werner Imseng, Marienhof, Saas-Fee aus: Ruppen et al.1988).

Noch nicht abgeschlossen sind die Arbeiten an einem umfassenden Oral-HistoryProjekt zur Waldnutzungsgeschichte in der Schweiz, in dem in ausgewählten Untersuchungsregionen ein präzises Bild der Waldnutzugsgeschichte seit dem 2. Weltkrieg gewonnen werden soll.

Diese Projekte illustrieren das große Potential von Oral-History-Interviews als Werkzeug für die Sicherung des Wissens um die Landnutzungsgeschichte (Fogerty 2001) und deuten auf der inhaltlichen Ebene an, wie wichtig dieses Wissen als Grundlage für das Verständnis anthropogen geprägter (Wald-)Ökosysteme ist.

Anwendung: Landnutzung als anthropogener Störungseinfluss auf die Ökosysteme

Seit mehreren tausend Jahren beeinflusst der Mensch die Vegetation in Europa durch Rodung, Beweidung, das Anlegen von Äckern und Gärten und durch die Einführung von Kulturpflanzen und der damit einhergehenden Begleitflora. Viele Arten wanderten im Zuge der menschlichen Tätigkeiten aus mediterranen und osteuropäischen Steppengebieten ein, andere breiteten sich aus näher gelegenen natürlichen Habitaten wie Auen, alpinen Rasen oder Uferzonen in die Kulturlandschaft aus (Landolt 1991). Schätzungen für die Schweiz gehen dahin, dass etwa 
700 der insgesamt 2.700 Gefässpflanzen ihr Vorkommen in der Schweiz menschlichen Aktivitäten verdanken. Für kleinere, intensiv genutzte Regionen sinkt der Anteil der natürlichen Artenvielfalt auf unter 50\% (vgl. ebd.). Die Zahlen für Deutschland scheinen sich in der ähnlichen Größenordnung zu bewegen (vgl. Korneck et al. 1998). Ein durchaus bedeutender Anteil der Arten in anthropogen stark geprägten Regionen sind somit Kulturfolger.

Diese Entwicklung fand selbstverständlich auch im Wald statt. In einem Wald, der als Teil der bäuerlichen Kulturlandschaft bewirtschaftet wurde, war auch das Habitatsangebot für Tiere und Pflanzen durch eben diese bäuerlichen Aktivitäten geprägt. Agrarische Waldnutzungen wurden nicht zentral definiert oder geplant, sondern sie entstanden aus den unterschiedlichen Bedürfnissen in Wechselwirkungen mit den lokalen naturräumlichen Gegebenheiten. Entsprechend groß war die Vielfalt der lokalen Ausprägungen von Nutzungsarten und der dadurch geschaffenen Habitate. Dennoch lassen sich einige Hauptnutzungsweisen unterscheiden. Erwähnt seien die Waldweide, die Waldheunutzung, das Sammeln von Streue, Nadel- und Laubfutter, der Waldfeldbau und eine ausgeprägte Sammelwirtschaft (Stuber u. Bürgi 2001, 2002; Bürgi u. Stuber 2003).

Um den Einfluss dieser Nutzungsweisen auf die Waldentwicklung abzuschätzen, muss er in Relation gesetzt werden zu den übrigen Einflüssen auf den Wald wie Waldbrand, Insektenkalamitäten, natürliche Sukzession oder atmosphärische Deposition von Stickstoffverbindungen oder Schadstoffen. Allerdings liegen nur wenige quantitative Angaben zu Veränderungen der nutzungsbedingten Einflüsse auf die Nährstoffversorgung der Waldpflanzen vor. Sicher ist, dass durch die agrarischen Waldnutzungsformen beträchtliche Mengen an Biomasse und damit Nährstoffen aus den Wäldern entfernt wurden. Doch wieviel? Basierend auf den in dem erwähnten Projekt zur Nutzungsgeschichte der Waldföhrenwälder gesammelten Angaben zu Waldweide und Waldstreunutzung (Gimmi u. Bürgi 2007) wurde versucht, diese Nutzungseinflüsse für den Walliser Wald zu quantifizieren (Gimmi et al. 2008). Bezüglich der Waldstreunutzung galt es in einem ersten Schritt, die wesentlichen Einflussgrössen zu bestimmen. Diese umfassten einerseits Faktoren, die die Nachfrage nach Waldstreue bestimmten, und andererseits Faktoren mit Auswirkungen auf die raum-zeitliche Verteilung der Nachfragebefriedigung, d.h. der effektiven Nutzung. In der Sprache der Ökologie wäre letztere als anthropogene Störung des Waldökosystems zu bezeichnen. Neben der Art und Anzahl der Nutztiere, der Dauer der Stallhaltung, der Verfügbarkeit von Alternativen zur Waldstreue (wie beispielsweise Stroh aus der lokalen Getreideproduktion und Schnittgut von lokalen Streuwiesen) war auch die ökonomische Situation der Bauern zu berücksichtigen, da diese bestimmte, ob Alternativen zur Waldstreue auch tatsächlich eingekauft werden konnten (insb. Getreidestroh von außerhalb der Gemeinde) (vgl. Abb. 4). Für die Bestimmung der raum-zeitlichen Verteilung der Waldstreunutzung galt es, die entsprechenden rechtlichen Bestimmungen, die Angaben über die tatsächliche Verbreitung, die Topographie und die Baumartenverteilung zu berücksichtigen. Letztere war insofern wichtig, als sich die Blatt- und 
Nadelstreue der verschiedenen Baumarten unterschiedlich für die Einstreuung in den Ställen eignete.

\section{Forest litter collecting}

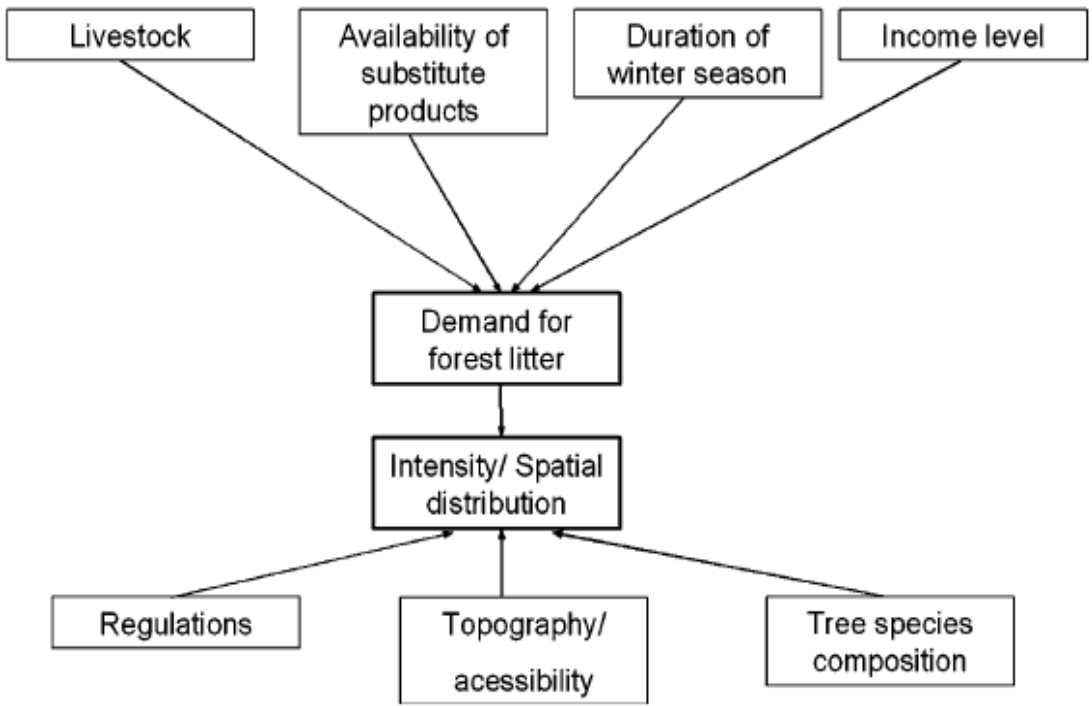

Abb. 4: Konzeptionelle Darstellung der Faktoren mit Einfluss auf die Nachfrage nach Waldstreue und der raum-zeitlichen Verteilung der Waldstreunutzung (aus: Gimmi et al. 2008)

Basierend auf diesen Angaben war es möglich, die Entwicklung der Nachfrage nach Waldstreue für die Periode von 1917 bis $1960 \mathrm{zu}$ bestimmen (Details in Gimmi et al. 2008). Es zeigte sich, dass ein wesentlicher Teil der Wälder im Untersuchungsgebiet durch Waldstreunutzung und/oder Waldweide genutzt worden war. Zudem zeigte sich, dass diese Nutzungsweisen nicht kontinuierlich zurückgegangen waren, sondern in den Jahren des zweiten Weltkrieges erneut an Bedeutung gewonnen hatten. Die Resultate dieser Studie wurden als weiteres Indiz für die große Bedeutung der Umweltgeschichte für ein vertieftes Verständnis der Entwicklung von Ökosystemen in der Kulturlandschaft interpretiert.

\section{Umsetzung: Entwicklung neuer Instrumente für den Naturschutz}

Die Veränderung der Vegetation und der Artenvielfalt in Schweizer Wäldern ist Thema mehrerer jüngerer Publikationen (Literatur in Wohlgemuth et al. 2002). Dabei werden die Veränderungen durch den Vergleich von früheren und heutigen Artenlisten untersucht. In einem solchen Vergleich der heutigen Flora mit jener vor 100 Jahren wurde für den Hügelzug der Lägern im Kanton Aargau das Verschwinden von 13 Waldpflanzen sowie ein starker Populationsrückgang für weite- 
re 10 früher im Wald wachsende Arten dokumentiert (Egloff 1999). Als Grund für diese Entwicklung wurde die zunehmende Verdunkelung der Waldbestände genannt. Für Waldpflanzen im Kanton Aargau wurde - ebenfalls für die letzten 100 Jahre - eine Aussterbensrate von 4 bis $8 \%$ berechnet. Walther und Grundmann (2001) kamen zu ähnlichen Resultaten, als sie entlang eines Transektes von Schaffhausen bis ins Tessin total 37 Standorte mit Vegetationsaufnahmen aus dem Zeitraum 1940 bis 1965 erneut vegetationskundlich erhoben. Dabei wurde ein mittlerer Verlust von 15 Gefäßpflanzenarten (von 41,0 auf 25,9) pro Aufnahme ermittelt. In nur 5 der 37 Aufnahmen stieg die Artenzahl. Ein Vergleich der mittleren Zeigerwerte erbrachte eine Abnahme der Licht-Zahl, d.h. eine Abnahme der Halbschatten- und Saumpflanzen, und eine Zunahme der Nährstoff- und Temperaturzahlen. Der Rückgang von Magerkeitszeigern und die Zunahme von Nährstoffzeigern sind Indizien für die Erhöhung des Nährstoffangebots (Kuhn 1993; Walther und Grundmann 2001). Die Ursachen hierfür können erhöhte atmosphärische Nährstoffeinträge sein. Gleichzeitig führte jedoch auch die erwähnte Aufgabe von agrarischen Waldnutzungen zu einem Anstieg der Nährstoffvorräte in den Waldböden, denn diese Nutzungsweisen waren generell mit einem Export von Biomasse in verschiedener Form verbunden. Doch welches sind die hauptsächlichen Ursachen der beobachteten Veränderungen? In welchen Anteilen sind Verdunkelung, Klimaerwärmung, höhere $\mathrm{CO}_{2}$-Konzentrationen und Veränderungen in den Keimbedingungen zusätzlich beteiligt? Hier sind weitere, systematische Untersuchungen nötig.

Um dem beobachteten Rückgang von Halbschattenpflanzen in zuwachsenden Wäldern entgegen zu wirken und so die Artenvielfalt zu fördern, wurden in den letzten Jahren in verschiedenen Schweizer Kantonen Auflichtungen in den Wäldern vorgenommen (z.B. Fachstelle Naturschutz 2001). Auflichtungen beeinflussen natürlich in erster Linie den Faktor „Licht“. Wie verhält es sich aber mit dem Faktor „Nährstoff“? Könnte die gezielte Wiedereinführung von Austragsnutzungen, beziehungsweise der damit verbundene Austrag an Nährstoffen, ebenfalls der Artenvielfalt förderlich sein?

Wenn keine quantitativen Angaben einen vermuteten Sachverhalt belegen können, hilft das Experiment. In einer Erfolgskontrolle hat der Kanton Zürich die regelmäßige Entfernung von Laubstreue bei einer Auflichtungsmaßnahme seit 1994 beobachtet und in ihren Folgen für die Waldpflanzen als positiv beurteilt (vgl. Fachstelle Naturschutz 2001). Streunutzungs-Experimente wurden weiter in Kiefernwäldern Niederbayerns durchgeführt (Beer u. Ewald 2005; Prietzel u. Kaiser 2005), und Hofmeister et al. (2004) gingen der Frage nach, welche Rolle die Aufgabe der Streunutzung bei der beobachteten Ausbreitung der Esche in europäischen Eichenwäldern spielt. Eine polnische Studie zeigte kürzlich, dass mit der periodischen Entfernung der Laubstreue die Artenvielfalt in der Krautschicht wesentlich gefördert werden kann (Dzwonko und Gawronski 2002). Die Autoren dieser Studie folgerten, dass die Zunahme von Nährstoffzeigern in der Krautschicht auf die Aufgabe der traditionellerweise praktizierten Entfernung der 
Laubstreue zurückzuführen ist. Die verbesserten Keimbedingungen auf laubfreiem Boden werden als Hauptgrund für die Zunahme der Moos- und Pflanzenvielfalt gesehen. Eine Veränderung der Nährstoffversorgung kann nach wenigen Jahren der Streuentfernung noch nicht erwartet werden. Auch bei einem allfälligen Rückgang der atmosphärischen Nährstoffeinträge durch Massnahmen im Bereich der Luftreinhaltung wird aufgrund der ausbleibenden Laubstreunutzung der Trend zur Eutrophierung der Wälder weiter gehen.

Mit dieser Ausgangslage beschlossen verschiedene Forschungsgruppen an der WSL in Birmensdorf, Schweiz, die Auswirkung der Entfernung der Laubstreue auf unterschiedlichen Standorten zu untersuchen. In 15 verschiedenen Buchenbeständen an der Lägern, am Irchel und am Sanzerberg bei Bachs (alle Standorte liegen im Zürcher Unterland) wurden Paare von $100 \mathrm{~m}^{2}$ großen Dauerflächen eingerichtet. Jeweils auf der Experimentfläche wird am Ende des Winters alles Laub zusammen gerecht. Die benachbarte, rund drei Meter entfernte Kontrollfläche bleibt dagegen unbehandelt, bzw. das anfallende Laub bleibt dort liegen. Alle Dauerflächen wurden 2003, vor dem ersten Laubaustrag, vegetationskundlich erhoben. Seither werden die Experimentflächen jedes Jahr gerecht und die Vegetation jeweils im Sommer erhoben (Bürgi et al. 2006). Drei Paare der Dauerflächen werden bodenkundlich untersucht. Vor dem ersten Laubaustrag wurden Oberbodenproben zur Charakterisierung des Säuregrades und des Nährstoffzustandes entnommen. Diese Beprobung wird während der Laufzeit des Projektes noch zwei Mal wiederholt. Zusätzlich wurden Entnahmestellen für Bodenwasser installiert, an welchen vier Mal im Jahr Bodenwasser gewonnen und die darin enthaltenen Nährstoffe analysiert werden.

Die Laufzeit des Experimentes ist auf 10 Jahre festgelegt. Noch ist es zu früh, um Resultate vorzustellen. Unsere Ausführungen zeigen aber grundsätzlich, dass es sich bei der Ausgestaltung moderner Naturschutzmaßnahmen im Wald lohnt, die Konsequenzen früherer Nutzungsformen zu bedenken. Große Artenvielfalt im Wald war oftmals mit agrarischer Nutzungsweise verbunden. Die regelmäßige Mahd von Trocken- und Feuchtwiesen - eine heute akzeptierte Naturschutzmaßnahme im Offenland - entspricht vom Prinzip her einer regelmäßigen Laubentfernung in Mittellandwäldern und stellt damit eine anregende Analogie dar.

\section{Folgerungen und Ausblick}

Um dem Anspruch einer historisch-ökologischen Sicht auf Landnutzungsgeschichte und Ökosystementwicklung gerecht zu werden, benötigt die Historische Ökologie möglichst genaue Angaben zu den relevanten menschlichen Tätigkeiten. Sie pflegt daher intensive Kontakte mit der Wald- und Forstgeschichte, der Umweltgeschichte und der Agrargeschichte. Zudem ist vor dem Hintergrund der großen Nachfrage nach präzisen und nach Möglichkeit quantifizierten Angaben zu 
den menschlichen Nutzungseinflüssen auf Ökosysteme an hohen Standards bezüglich quellenkritischem Arbeiten festzuhalten.

Die vorgestellten Untersuchungen zeigen, dass mittels Oral-History-Interviews ein wichtiger Beitrag zur Sicherung und Dokumentation des kulturellen Erbes geleistet werden kann. Zugleich kann die Quellenlage zu den relevanten Nutzungen verbessert werden. Basierend auf derartigen Informationen kann die Analyse der Dynamik anthropogener Landschaften und Ökosysteme verbessert werden. Der Grad der Anwendbarkeit und Praxisrelevanz der so gewonnenen Einsichten mag von Projekt zu Projekt sehr unterschiedlich sein. Nicht immer ist der Anspruch, Handlungswissen zu generieren, sinnvoll einzuhalten. In den meisten Fällen ist es jedoch durchaus angebracht, durch gezielte Publikationstätigkeit die Resultate historisch-ökologischer Studien der Praxis im Bereich Forstwirtschaft oder Landschaftsplanung, aber auch einer breiteren Öffentlichkeit zugänglich zu machen. 


\section{Literatur und Quellen}

Allen, T. F. H., Tainter, J. A., Pires J. C. u. Hoekstra, T. W. (2001): Dragnet ecology - ,just the facts, ma'am": the privilege of science in a postmodern world. BioScience 51: 475-485.

Balée, W. (Hrsg.) (1998): Advances in historical ecology. Columbia University Press, New York.

Beer, A. u. Ewald, J. (2005): Vegetationskundliche Untersuchungen rezent genutzter Kiefernwälder auf Binnendünen des niederbayerischen Tertiärhügellandes. Tuexenia 25: 93-109.

Bürgi, M. (2003): Historische Ökologie im und um den Wald. Schweizerische Zeitschrift für Forstwesen 154: 328-332.

Bürgi, M. u. Gimmi, U. (2007): Three objectives of historical ecology: the case of litter collecting in Central European forests. Landscape Ecology 22: 77-87.

Bürgi, M., Russell, E. W. B. u. Motzkin, G. (2000): Effects of post-settlement land-use history on forest composition in the north-eastern United States - a comparative approach. Journal of Biogeography 27: 1123-1138.

Bürgi, M. u. Stuber, M. (2003): Agrarische Waldnutzungen in der Schweiz 18001950. Waldfeldbau, Waldfrüchte und Harz. Schweizerische Zeitschrift für Forstwesen 154: 360-375.

Bürgi, M., Wohlgemuth, T. u. Zimmermann, S. (2006): Austragsnutzungen im Wald. Zürcher Wald 5: 28f.

Christensen, N. L. (1989): Landscape history and ecological change. Journal of Forest History 33: 116-125

Costanza, R., Graumlich, L., Steffen, W., Crumley, C., Dearing, J., Hibbard, K., Leemans, R., Redman, C. u. Schimel, D. (2007): Sustainability or collapse: what can we learn from integrating the history of humans and the rest of nature? Ambio 36: 522-527.

Crumley, C. L. (1994): Historical ecology: Cultural knowledge and changing landscapes. School of American Research Press, Santa Fe.

Dambrine, E., Dupouey, J.-L., Laut, L., Humbert, L., Thinon, M., Beaufils, T. u. Richard, H. (2007): Present forest biodiversity patterns in France related to former Roman agriculture. Ecology 88: 1430-1439.

Dearing, J.A., Battarbee, R.W., Dikau, R., Larocque, I. u. Oldfield, F. (2006): Human-environment interactions: towards synthesis and simulation. Reg Environ Change 6: 115-123. 
Dirkx, J. (2004): Historical ecology of Dutch cultural landscapes: references for the integration of landscape planning and nature restoration in the Netherlands. In: Brandt, J., Vejre, H. (Hrsg.): Multifunctional landscapes. I: Theory, values and history WIT Press, Southampton Boston, S. 137-149.

Dzwonko, Z. u. Gawronski, S. (2002): Effect of litter removal on species richness and acidification of a mixed oak-pine woodland. Biological Conservation 106: 389-398.

Edmonds, M. (2001): The pleasures and pitfalls of written records. In: Egan, D., Howell, E.A. (Hrsg.): The historical ecology handbook. Island Press, Washington, S. 73-99

Egan, D. u. Howell, E.A. (Hrsg.) (2001): The historical ecology handbook. Island Press, Washington DC.

Egloff, F. G. (1999): Dauer und Wandel der Lägernflora. Vierteljahrsschr. Naturf. Ges. Zürich 136: 207-270.

Fachstelle Naturschutz Kanton Zürich (2001): Lichter Wald - Ergebnisse aus Erfolgskontrollen. Fachstelle Naturschutz, Zürich.

Fogerty, J. E. (2001): Oral history: a guide to its creation and use. In: Egan, D., Howell, E.A. (Hrsg.) The historical ecology handbook. Island Press, Washington, S. 101-120.

Forman, R. T. T. u. Russell, E.W.B. (1983): Evaluation of historical data in ecology. Bulletin Ecological Society America 64: 5-7.

Foster, D., Swanson, F., Aber, J., Burke, I., Brokaw, N., Tilman, D. u. Knapp, A. (2003): The importance of land-use legacies to ecology and conservation. BioScience 53: 77-88.

Foster, D. R. u. Motzkin, G. (2003): Interpreting and conserving the openland habitats of coastal New England: insights from landscape history. Forest Ecology and Management 185: 127-150.

Gimmi, U. u. Bürgi, M. (2007): Using oral history and forest management plans to reconstruct traditional non-timber forest uses in the Swiss Rhone Valley (Valais). Environment and History 13: 211-246.

Gimmi, U., Bürgi, M. u. Stuber, M. (2008): Reconstructing anthropogenic disturbance regimes in forest ecosystems - a case study from the Swiss Rhone valley. Ecosystems 11: 113-124.

Goudie, A. (1981): The human impact on the natural environment. Blackwell, Malden.

Hall, M. (2005): Earth repair - a transatlantic history of environmental restoration. University of Virginia Press, Charlottesville and London. 
Hellberg, E., Hörnberg, G., Östlund, L. u. Zackrisson, O. (2003): Vegetation dynamics and disturbance history in three deciduous forests in boreal Sweden. Journal of Vegetation Science 14: 267-276.

Hessburg, P. E. u. Agee, J. K. (2003): An environment narrative of Inland Northwest United States forests, 1800-2000. Forest Ecology and Management 178: 23-59.

Hessl, A. (2002): Aspen, elk and fire: the effects of human institutions on ecosystem processes. BioScience 52: 1011-1022.

HITE (2006): HITE research plan. http://www.liv.ac.uk/geography/ research_projects/Hite/Scipaln.htm. (6. Mai 2008).

Hofmeister, J., Mihaljevic, M. u. Hosek, J. (2004): The spread of ash (Fraxinus excelsior) in some European oak forests: an effect of nitrogen deposition or successional change?. For. Ecol. Manage. 203: 35-47.

Korneck, D., Schnittler, M., Klingenstein, F., Ludwig, G., Takla, M., Bohn, U. u. May, R.. (1998): Warum veramt unsere Flora? Auswertung der Roten Liste der Farn- und Blütenpflanzen Deutschlands. Schriftenreihe für Vegetationskunde 29: 299-444.

Kuhn, N. (1993): Ursachen floristischer und ökologischer Vorgänge in Waldbeständen. Schweiz. Z. Forstwes. 144: 347-367.

Küster, H. (1995): Geschichte der Landschaft in Mitteleuropa. C.H. Beck, München.

Landolt, E. (1991): Gefährdung der Farn- und Blütenpflanzen in der Schweiz mit gesamtschweizerischen und regionalen roten Listen. Eidgenöss. Drucksachen- und Materialzentrale (EDMZ), Bern. 185 S.

Marcucci, D. J. (2000): Landscape history as a planning tool. Landscape and Urban Planning 49: 7-81.

Marsh, G. P. (2003): Men and nature. (1864) reprint edited by Lowenthal D. University of Washington Press, Seattle London.

Nassauer, J. I. (1995): Culture and changing landscape structure. Landscape Ecology 10: 229-237.

Östlund, L., Zackrisson, O. u. Axelsson, A. L. (1997): The history and transformation of a Scandinavian boreal forest landscape since the 19th century. Canadian Journal of Forest Research 27: 1198-1206.

Prietzel, J. u. Kaiser, K. O. (2005): Vegetationskundliche Untersuchungen rezent genutzter Kiefernwälder auf Binnendünen des niederbayerischen Tertiärhügellandes. J. Plant Nutr. Soil Sci 168: 461-471.

Roth, L. u. Bürgi, M. (2006): Bettlaubsammeln als Streunutzung in St. Galler Rheintal. Schweizerische Zeitschrift für Forstwesen 157: 348-356. 
Ruppen, P. J., Imseng, G. u. Imseng, W. (1988): Saaser Chronik 1200-1988. SaasFee.

Russell, E. W. B. (1997): People and the land through time: linking ecology and history. Yale University Press, New Haven.

Schenk, W. (2006): Historische Kulturlandschaftsforschung im Spannungsfeld von älteren Ansätzen und aktuellen Fragestellungen und Methoden. Siedlungsforschung 24: 9-12.

Simmons, I. G. (1989): Changing the face of the earth. Culture, environment, history. Basil Blackwell, Oxford Cambridge.

Stebler, F. G. (1922): Die Vispertaler Sonnenberge. In: Jahrbuch des Schweiz. Alpen-Club 56, Bern.

Stuber, M. u. Bürgi, M. (2001): Agrarische Waldnutzungen in der Schweiz 18001950. Waldweide, Waldheu, Nadel- und Laubfutter. Schweizerische Zeitschrift für Forstwesen 152: 490-508.

Stuber, M. u. Bürgi, M. (2002): Agrarische Waldnutzungen in der Schweiz 18001950. Nadel- und Laubstreue. Schweizerische Zeitschrift für Forstwesen 153: 397-410.

Thomas, W. L. (Hrsg.) (1956): Man's role in changing the face of the earth. University of Chicago Press.

Walther, G. R. u. Grundmann, A. (2001): Trends of vegetation change in colline and submontane climax forests in Switzerland. Bull. Geobot. Inst. ETH 67: 312.

Winiwarter, V. (2007): Einstieg in die Umweltgeschichte. In: Winiwarter, V., Knoll M. Umweltgeschichte. UTB 2521, S. 19-42.

Wohlgemuth, T., Bürgi, M., Scheidegger, C. u. Schütz, M. (2002): Dominance reduction of species through disturbance - a proposed management principle for central European forests. Forest Ecology and Management 166: 1-15.

http1: http://www.unesco.org/mab/ <06.05.2009>

http2: http://lternet.edu/ <06.05.2009>

http3: http://www.pages.unibe.ch/ <06.05.2009> 
Historische Wassermühlen in Südniedersachsen

Esben Eidevik 


\begin{abstract}
Since the Middle Ages, water mills have had a continuing influence on everyday life in Central Europe. In the 20th century, an increasing number of water mills were replaced by motor-driven mills. Today a great number of former mill buildings have been converted to other uses, especially popular is the conversion to residental houses. In order to protect the cultural heritage, several organizations are trying to integrate still existing mills or at least their buildings into a tourist oriented concept.

The first step requires mapping their location, so the author has started mapping structures in the area surrounding Göttingen, following the Leine river. The author has listed the mills and their location. This list gives us an indication of the most important aspects that should be incorporated into a tourist concept. For example of tourist value is the age of some of the mills, since they were founded in medieval times.

Another interesting point is the period of "Mühlensterben", the time when an increasing number of mills were shut down. The different types of mills that formerly existed in the mapped area, could be described, as well. A large number of former mills today have a turbine and generator to produce electricity. This example of timeless (mill) technology should also play an important role in a touristic concept.
\end{abstract}

\title{
Einleitung
}

Seit der Antike nutzt der Mensch mit Mühlen Wind- und Wasserkraft gezielt aus, um sie auf Mahlwerke oder andere Apparaturen zu übertragen. Mühlen stellen damit die älteste Möglichkeit für den Menschen dar, Naturkräfte umzuwandeln und für sich nutzbar zu machen (vgl. Löbert 2001, S. 7).

Bis ins 20. Jahrhundert hinein haben Mühlen das Leben der Menschen auf vielfältige Art beeinflusst. In der ersten Hälfte des vergangenen Jahrhunderts sind die meisten Mühlen in Niedersachsen stillgelegt worden, zum Teil sind sogar die Gebäude verschwunden. Nur ein Bruchteil der Betriebe konnte bis heute erhalten werden. Mittlerweile gibt es eine beträchtliche Anzahl historischer Mühlen, die touristisch in Wert gesetzt und so einer neuen Nutzung zugeführt worden sind. Die touristische Nutzung stellt eine vielversprechende Möglichkeit dar, die notwendigen finanziellen Mittel zur Erhaltung der Mühlen aufzubringen.

Das Projekt der „Mühlenstraße Niedersachsen“ hat hierzu die Mühlen der Landkreise Lüneburg, Harburg, Lüchow-Dannenberg, Uelzen und Celle kartiert. Mit Hilfe finanzieller Unterstützung durch die Europäische Union und beteiligter Landkreise konnten einige Mühlen restauriert werden. Sehens- und schützenswer- 
te Mühlen wurden anschließend durch einen ausgeschilderten Radwanderweg miteinander vernetzt, so dass Interessierte die noch vorhandenen (und zugänglichen) Mühlen besichtigen können (vgl. Löbert 2001, S. 7).

Für Südniedersachsen fehlt ein derartiges Kataster, für diesen Raum existiert derzeit nur eine frühere Bestandsaufnahme von Wilhelm Kleeberg (Stand: 1961), die der Erfassung aller Mühlen dienen sollte und folglich keinen touristischen Verwertungszusammenhang beabsichtigte.

Im Rahmen der Examensarbeit des Autors wurde eine kulturlandschaftliche Aufnahme von Mühlen im südlichen Niedersachsen für das Jahr 2007 durchgeführt. Grob dem Lauf der Leine folgend wurde die Göttinger Umgebung erfasst und erhaltene Objekte auf ihre Eignung für eine touristische Nutzung untersucht. Im Anschluss daran wurden Vorschläge erarbeitet, auf welche Art die Mühlen touristisch in Wert gesetzt und - nach dem Vorbild der „Mühlenstraße Niedersachsen“ - vernetzt werden könnten.

Die Literatur zum Thema „Touristische Inwertsetzung historischer Mühlen“ ist uneinheitlich, da sie immer nur einen Teil der Thematik beleuchtet. Die Literatur zu Tourismus und Freizeit ist hingegen umfangreich, es werden die Akteure des Tourismus sowie seine Markt- und Organisationsstrukturen untersucht (vgl. Mundt 1998; Steinbach 2003). Greift man den Teilbereich Kulturtourismus heraus, werden zwar Besichtigungsziele wie Schlösser, Burgen etc. erwähnt (vgl. Heinze 1999), jedoch nicht explizit touristisch genutzte Mühlen, die als Baudenkmale zu dieser Gruppe gerechnet werden können. Auch im Rahmen der zunehmenden Individualisierung des Angebotes und der verstärkten Erlebnisorientierung gerade des Kulturtourismus (vgl. Heinze 1999; Steinecke 1999) werden Mühlen nicht berücksichtigt. Allenfalls in einem Nebenzweig des Kulturtourismus, dem Industrietourismus, finden Mühlen Erwähnung (z.B. bei Schröder 2003, S. 213; nicht bei Wolf 2005). Die Einordnung der Mühlen in den Industrietourismus betrifft jedoch nur einen Teil des touristischen Potenzials von Mühlen, da ihre Nutzungsgeschichte viel weiter als die Industrialisierung zurückreicht und diese sich infolgedessen tiefer in der Vorstellungswelt der Menschen verankert hat (vgl. Büllesbach 1994).

In der Mühlenforschung wiederum stehen die Mühlen und die mit ihnen verbundenen Themengebiete im Mittelpunkt. Als technische Anlagen wurden Mühlen schon in der frühen Neuzeit mit ihrer Antriebstechnik und der Mahltechnik beschrieben, was heute im Rückblick vor allem unter dem Aspekt der Erhaltung und Instandsetzung geschieht (vgl. Kleeberg 1964; Wagenbreth et al. 1994). Gerade die Veröffentlichungen der republikweit tätigen Mühlenvereine thematisieren die materiellen Reste der Mühlen und fordern ihre Erhaltung (vgl. Thiemann et al. 2001). In ihnen wird die touristische Nutzung als eine der wichtigsten Möglichkeiten zur Beschaffung der finanziellen Mittel genannt, doch beschränken sich die Angebote auf Besichtigungen, Museen und gastronomische Einrichtungen (vgl. Arbeitsgruppe Mühlenstraße in der Mühlenvereinigung Niedersachsen-Bremen e.V. 1995). Eine darüber hinaus gehende touristische Inwertsetzung, insbesondere 
Historische Wassermühlen in Südniedersachsen

eine Hinwendung zu den im Tourismus angesprochenen Trends ist hier nicht zu erkennen. Auch die Aussparung der Industriemühlen, die mittlerweile zum Teil Denkmalstatus haben, ist nicht unumstritten (vgl. Bardua 2006).

Diese Zweiteilung der Literatur macht deutlich, dass eine auch an neueren Kundenwünschen orientierte touristische Inwertsetzung noch nicht erfolgt ist. Die Tourismusforschung müsste sich des durchaus vorhandenen Interesses an Mühlen annehmen und umgekehrt die Mühlenforschung die touristische Inwertsetzung stärker an den aktuellen Erfordernissen orientieren.

\section{Erfassung der Mühlen im Untersuchungsraum}

Aufbau der Untersuchung

Die Untersuchung gliedert sich in verschiedene Phasen. Zunächst wurde der Untersuchungsraum der Mühlenbesichtigung festgelegt, im Anschluss daran wurde ein Erfassungsbogen erstellt, um die historischen Mühlen aufzunehmen und vergleichbar zu machen.

Zur Auffindung der Mühlenstandorte im Leinetalgraben wurde das bereits erwähnte Mühlenkataster von Wilhelm Kleeberg (1964/1979) genutzt, da es das umfassendste Werk dieser Art für die Göttinger Umgebung darstellt.

Fokussiert wurde dabei der Leineberg in Göttingen und dessen Umland. Die Leinezuflüsse sollten dabei nicht grundsätzlich ausgeklammert werden, um potenziell für eine touristische Nutzung geeignete Mühlen an einem Leinezufluss nicht auszuschließen.

Da zur Durchführbarkeit der Besichtigung der Mühlen auch die Mobilität des Autors eine Rolle spielte, musste der Untersuchungsraum mit dem Fahrrad von Göttingen aus erreichbar sein, gegebenenfalls mit öffentlichen Verkehrsmitteln, die Fahrräder transportieren. Diese Einschränkung ist für den Vorschlag der touristischen Inwertsetzung unproblematisch, da in Anlehnung an die Überlegungen zur Niedersächsischen Mühlenstraße damit zu rechnen ist, dass das Angebot vor allem Radfahrer anspricht.

Der Untersuchungsraum (vgl. Abb. 1) erstreckt sich innerhalb der folgenden Grenzen: Im Süden beginnt die Aufnahme in Besenhausen und folgt dann der Leine über Niedergandern, Reckershausen, Friedland und Stockhausen. Auf Grund der räumlichen Nähe kann auch die Mühle in Obernjesa an der Dramme miterfasst werden. Danach folgt das Erfassungsgebiet der Leine über Niedernjesa nach Göttingen. Südlich von Göttingen fließt die Garte in die Leine, weshalb auch die Gartemühlen aufwärts bis Kerstlingerode beschrieben werden. Bei Göttingen werden die Mühlen der Leinezuflüsse Rase, Grone und Weende miterfasst. Danach folgt der Untersuchungsraum der Leine in nördlicher Richtung über Bovenden. Hier werden zusätzlich die Mühlen des Rauschenwassers, einen für seinen einstigen Mühlenreichtum bekannten Leinezufluss, aufgenommen. Der Leine 
folgend erstreckt sich das Aufnahmegebiet dann weiter über Nörten-Hardenberg (mit dem angrenzenden Rodetal) und Northeim bis Langenholtensen. In Northeim wird schließlkich auch ein Teil der Rhume miterfasst.

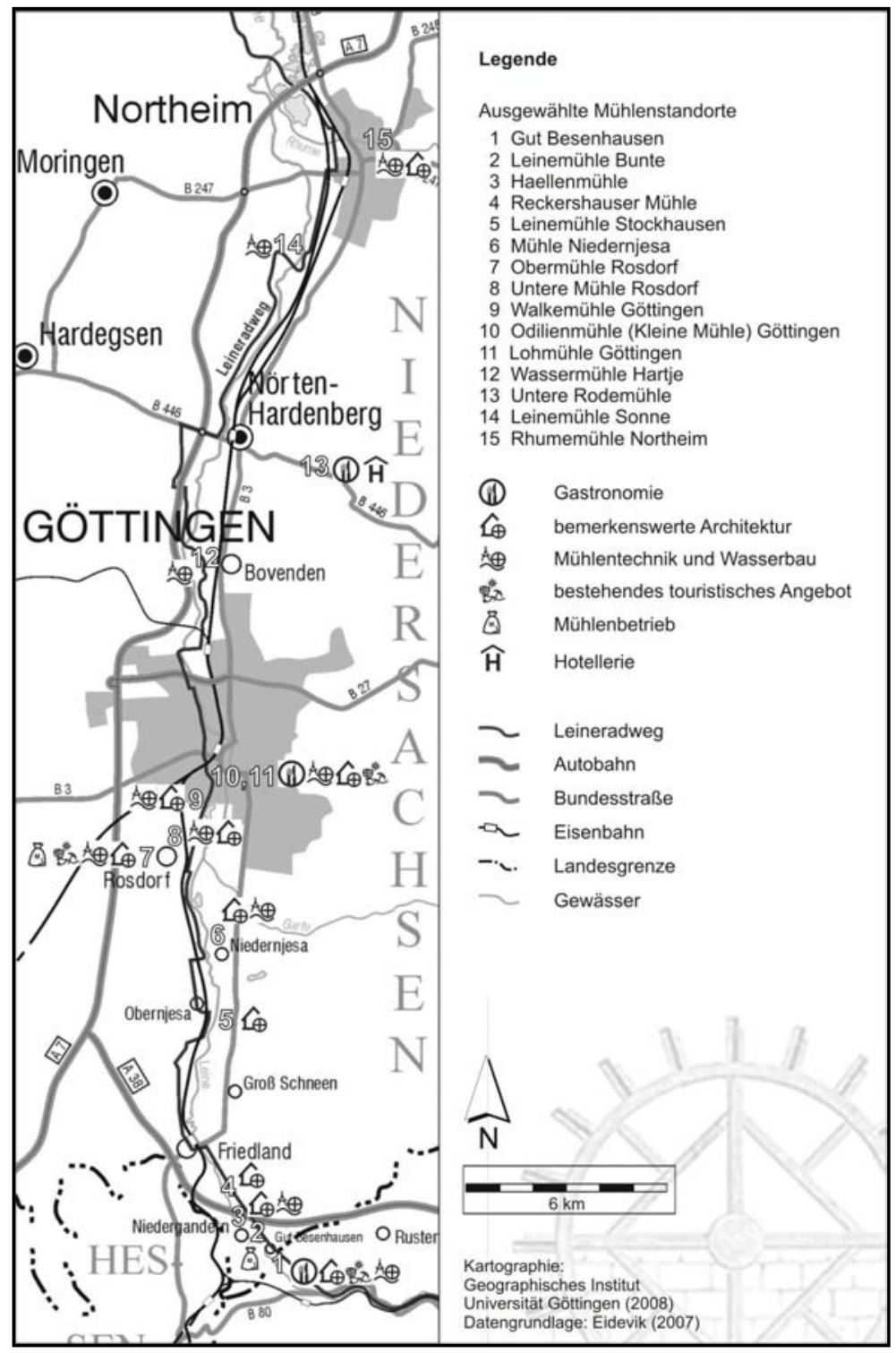

Abb. 1: Ausgewählte Mühlenstandorte im Untersuchungsraum 
Kriterien für die Beschreibung der Mühlen

In der Feldphase wurde die getroffene Auswahl der bei Kleeberg kartierten Mühlen angefahren und bewertet. Hierzu wurde ein Kriterienkatalog erarbeitet, um die Bewertung der Mühlen für den zu entwickelnden Wanderweg auf eine fundierte Grundlage zu stellen. Hierbei wurde versucht, gleichberechtigt die Funktionen der Mühlen als technische Anlagen, Landmarken und Denkmale zu berücksichtigen. Bei letztgenanntem Aspekt stützt sich der Erfassungsbogen auf die Interessen der Mühlendenkmalpflege nach Wagenbreth et al. (1994).

Der Erfassungsbogen ist auf eine Begutachtung des Objektes von außen ausgelegt. Eine Vielzahl der Untersuchungskriterien sollte mit einer Vergabe von Punkten nachvollziehbar gemacht werden. Die nicht in einem Punkteschema fassbaren Beobachtungen, etwa die Art des Mühlenrades bzw. der Turbine, wurden verbal-deskriptiv festgehalten. Mit Sonderpunkten wurde im Einzelfall ihrem Schauwert Rechnung getragen. Neben dem Mühlenbaukörper an sich wurden auch seine Umgebung und die Wirkung des Ensembles festgehalten.

Der Erfassungsbogen ist im Einzelnen wie folgt aufgebaut: Der Punkt Gebäudeäußeres umfasst alles am Gebäudekörper Erkennbare, das für eine Eingliederung in das Nutzungskonzept von Relevanz sein könnte. So lässt sich eine bereits in gutem Zustand befindliche Mühle mit geringerem Aufwand in ein touristisches Nutzungskonzept integrieren und erhält daher eine höhere Bewertung als ein baufälliges Objekt.

Der Baustil (Fachwerk, Backsteinmauern, Beton,...) wird primär deskriptiv erfasst. Sollte eine Mühle durch ihren Baustil über ein Alleinstellungsmerkmal verfügen, kann ihr hierfür ein Sonderpunkt gegeben werden. Auch etwaige (evtl. unsachgemäße) Veränderungen am Gebäude (z.B. Anbauten) werden erfasst. Unter Besonderheiten werden weitere erwähnenswerte Auffälligkeiten vermerkt.

Der Aspekt mühlenspezifische Merkmale dient dem Festhalten noch vorhandener Elemente, die auf die Funktion des Gebäudes als Mühle hinweisen. Wagenbreth et al. (1994, S. 180) stellen fest, dass selbst der scheinbare Betrieb einer Mühle ihre Aussagekraft für den Betrachter verstärkt: „Die historische Aussage und die landschaftliche Wirkung einer Windmühle sind selbstverständlich besser gegeben, wenn sie ein Flügelkreuz hat. [...] Das Anbringen von Flügelkreuzen ist ebenso gerechtfertigt, wenn ein Betrieb nicht vorgesehen ist. [...] Bei Wassermühlen sind analog Wasserräder zu rekonstruieren und möglichst (zumindest zeitweise) in Betrieb zu setzen. Dies ist auch im Leerlauf möglich. Die historische Aussage und die emotionale Wirkung eines sich drehenden Wasserrades sind größer als die eines ruhenden." Aus diesem Grund werden das Vorhandensein und insbesondere der Betrieb von Wasserrädern und Turbinen mit drei Pluspunkten bewertet. Liegen vorhandene Antriebsmaschinen still, erhält die Mühle einen Punkt. Entfernte Anlagen werden mit zwei Minuspunkten angerechnet. Hiermit wird verhindert, dass der Gebäudezustand höher bewertet wird als vorhandene mühlentypische Merkmale, die für die Mühle wichtiger sind. Sonstige Spuren der Nutzung als 
Mühle am Gebäude (Lettern, Mühlstein,...) können ebenfalls erfasst werden. Ein weiterer wichtiger Punkt sind die noch erkennbaren wasserbaulichen Maßnahmen für den Mühlenbetrieb (z.B. Mühlengraben, Mühlenteich, Stauwehr oder Gerinne), die abhängig von ihrem Zustand mit je einem halben oder ganzen Punkt bewertet werden können. Falls der Standort selbst besondere Aussagekraft besitzt, soll er ebenfalls festgehalten werden. Dies wäre der Fall etwa mit der Nähe zu einer weiteren Mühle (dies ließe die ehemalige Dichte des Mühlennetzes erahnen) oder als Zeuge eines wüst gefallenen Dorfes. Auch die etwaige Rolle, welche die Mühle in Sagen oder regionalem Brauchtum spielt, kann hier Berücksichtigung finden.

Die Kategorien des Punktes Lage zielen stark auf die mögliche touristische Nutzung der Mühle ab. Die Erreichbarkeit/Anbindung beschreibt die derzeitige Nähe zu (Fern-)Radwegen und Straßen sowie die Qualität der Wege dorthin. Gute und kurze Wege werden positiv bewertet. Die derzeitige Zugänglichkeit umfasst alle auf dem Grundstück der Mühle selbst vorgefundenen Gegebenheiten. Ist der Weg zur Mühle abgesperrt oder ist sie nicht zugänglich, führt dies zu einem Minus-, ein offenkundig freier Zugang zu einem Pluspunkt. Eine gute Sichtbarkeit zumindest von außerhalb des Grundstücks fließt mit einem halben Punkt in die Bewertung ein.

Die nähere Umgebung wird auf zwei Arten bewertet. Visuell wird der ästhetische Eindruck des Mühlengebäudes und seiner Umgebung festgehalten. Die Mühlen können bei diesem Punkt keine Abzüge bekommen. Hebt die Umgebung die Mühle gut hervor und/oder bereichert das Gebäude, können zwei Pluspunkte vergeben werden. Eine neutral-angemessene Umgebung, welche das Mühlengebäude nicht stört, führt zu einem Pluspunkt. Bei einer störenden optischen Wirkung der Umgebung auf die Mühle (etwa durch einen großflächigen Asphaltparkplatz) gibt es keine Punkte. Analog hierzu wird auch die Geräuschkulisse erfasst und bewertet. Liegt die Mühle in einer ruhigen Umgebung, so dass typische Geräusche wahrgenommen werden können (wie das Rauschen des Wassers), führt dies zu einer Bewertung mit zwei Punkten. Eine geringe Lärmbelastung führt zu einem Punkt, störender Lärm zu null Punkten in der Bewertung.

Abschließend wird unter dem Punkt Lage auch das Angebot ergänzender Produkte oder Dienstleistungen erfasst, etwa Versorgungsbetriebe wie eine Gaststätte oder eine Jugendherberge, für Bildungstouristen interessante Angebote wie ein thematisch passendes Museum oder einen Lehrpfad sowie spezielle Aktivitäten für Kinder, z.B. ein Abenteuerspielplatz. Jede Attraktion in relativer Nähe der Mühle kann mit nicht vorher festgelegten Sonderpunkten bedacht werden.

Die derzeitige Nutzung ist der letzte Punkt des Kriterienkataloges. Die Nutzungsart soll formal festgehalten werden. Mit dem Einfluss der derzeitigen Nutzung auf die kulturhistorische Aussagekraft der Mühle wird ein wichtiger Aspekt im Hinblick auf eine mögliche touristische Nutzung quantifiziert, da zu erwarten ist, dass bauliche Veränderungen an der Mühle mit der aktuellen Nutzung zusammenhängen. 


\section{Ersterwähnung und Stilllegung}

Nach Abschluss der Feldphase wurden die Erfassungsbögen ausgewertet und verschiedene Ansätze für den thematischen Rahmen einer touristischen Inwertsetzung herausgearbeitet.

Die Wassermühlen des untersuchten Gebietes sind unterschiedlichen Alters. Bei etlichen Mühlen ist nicht mehr feststellbar, wann sie eingerichtet wurden. Viele herrschaftliche Mühlen und Klostermühlen werden in Urkunden aus dem 14. Jahrhundert erstmals erwähnt, wobei Kleeberg in vielen Fällen anmerkt, dass das tatsächliche Alter der Mühlen höher sein dürfte. Für die Mühlen des Untersuchungsraumes ergibt sich folgendes Bild, wenn man die von Kleeberg (1964) genannten Daten der Ersterwähnung und die von den heutigen Eigentümern genannten Zahlen zusammenstellt:

Tab. 1: Erstmalige Erwähnung der Mühlen (Daten nach Kleeberg 1964/1979 sowie Angaben der heutigen Mühlenbesitzer)

\begin{tabular}{l|l}
\hline Vor dem 14. Jahrhundert & 3 \\
14. Jahrhundert & 9 \\
15.-18. Jahrhundert & 5 \\
20. Jahrhundert & 1 \\
unbekannt & 19 \\
\hline
\end{tabular}

Für das Nutzungskonzept ergibt sich daraus die Möglichkeit, die im Untersuchungsraum relativ häufig im Mittelalter gegründeten Mühlen herauszustellen. Dies könnte z.B. durch ein Herausgreifen der Klostermühlen oder herrschaftlichen Mühlen in Form eines Nebenweges oder einer besonderen Markierung auf der Wegekarte geschehen. Da Mühlen jedoch immer wieder versetzt oder zusammengelegt wurden, ist eine Kontinuität der Mühlenbetriebe über die Gebäude schwierig nachzuvollziehen. Des Weiteren wurden die ursprünglichen Gebäude oft durch Brände oder Kriege zerstört, so dass die Mühlengebäude ersetzt werden mussten, wie etwa im Fall der Langenholtenser Mühle (vgl. Kleeberg 1964, S. 189). Zum Teil wurden auch Gebäude auf Grund von Modernisierungen abgerissen und durch Neubauten ersetzt, etwa die Odilienmühle in Göttingen (vgl. Abb. 2), die 1305 erstmals urkundliche Erwähnung findet, deren heute erhaltenes Gebäude jedoch aus dem Jahr 1768 stammt. Die neuen Gebäude werden den Erfordernissen ihrer Entstehungszeit und einer eventuellen neuen Nutzung (z.B. einer anderen oder zusätzlichen Mühlenart) angepasst. Diese Entwicklung müsste dann ebenfalls im Nutzungskonzept thematisiert werden, wobei die Modernisierungen selbst bereits Teil der darzustellenden Mühlengeschichte sind. 


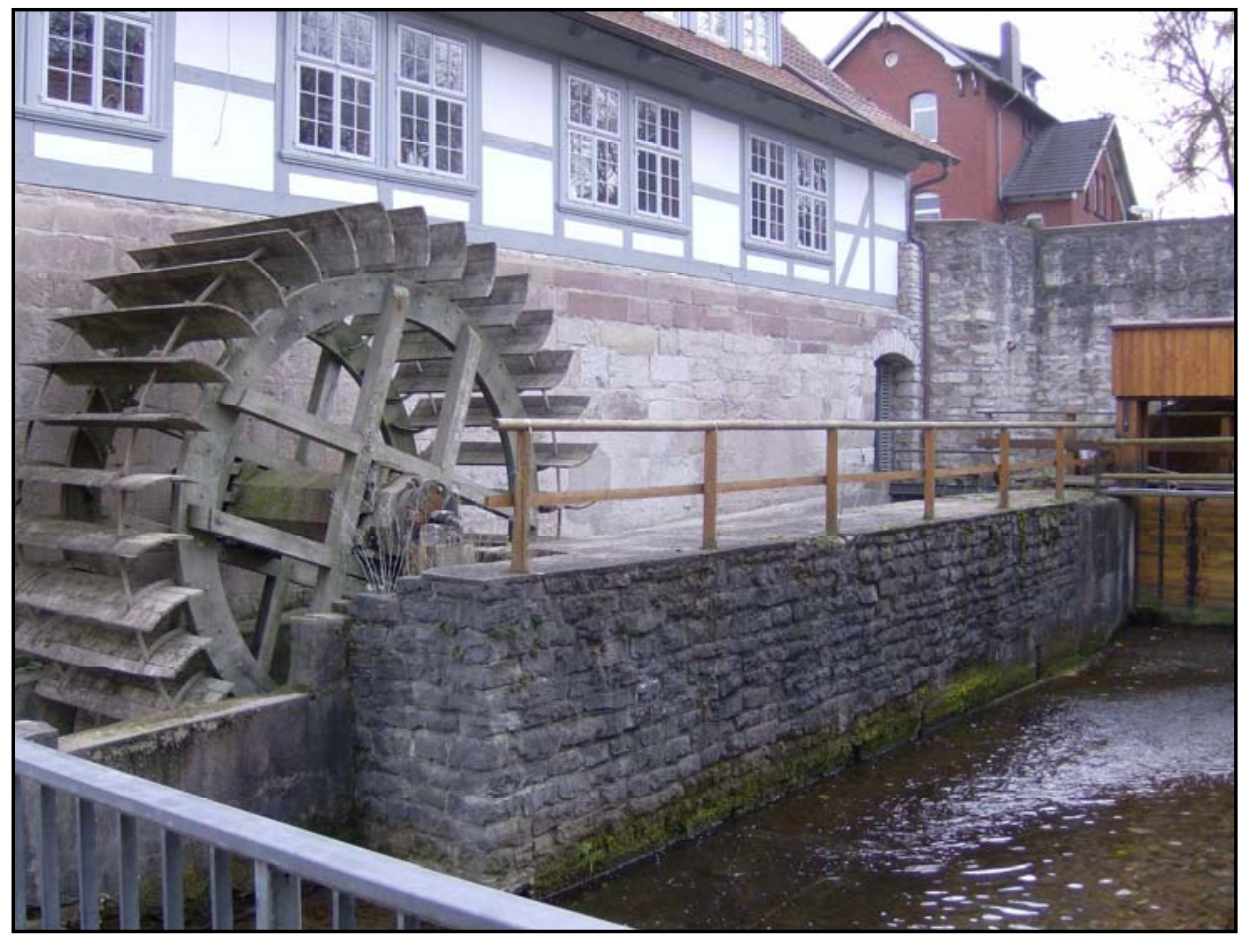

Abb. 2: Odilienmühle (Kleine Mühle) in Göttingen (Foto: Wieland)

Ein weiterer Punkt der Betrachtung ist das Jahr der Stilllegung. Ab diesem Zeitpunkt wurde die Mühle nicht mehr nach Gesichtspunkten ihres Betriebes verändert, sondern in der Regel umgenutzt. Veränderungen am Gebäude erfolgten in diesem Fall zu Gunsten der neuen Nutzungsform. Die Zeit, zu der die Mühle außer Dienst gestellt wurde, hat großen Einfluss auf ihre heutige Gestalt: Wurde noch eine Turbine installiert, wurden das Wasserrad und die dazugehörige Anlage entfernt, die für den Turbinenbetrieb nicht notwendig oder gar störend gewesen wären, z.B. das Gerinne. War das Wasserrad zum Zeitpunkt der Stilllegung einer Mühle jedoch noch vorhanden, so können sich mit größerer Wahrscheinlichkeit Spuren desselben finden lassen, die nicht beseitigt wurden, weil sie die neue Nutzung des Gebäudes und/oder der Anlage nicht störten. Dieser Zustand kann einen Hinweis darauf geben, welchen Zeitpunkt man als Ausgangspunkt für die touristische Inwertsetzung der Mühle wählt. Ein weiterer zu berücksichtigender Aspekt ist die Aussagekraft des Zeitpunktes der Stilllegung im Zusammenhang mit dem Phänomen des Mühlensterbens: Eine bereits im 19. Jahrhundert stillgelegte Mühle dürfte eine relativ geringe Leistung erbracht haben, leistungsfähigere Mühlen blieben noch bis in die erste Hälfte des 20. Jahrhunderts in Betrieb, in Ausnahmefällen wurden sie über die 1960er-Jahre hinaus betrieben. Die Verdeut- 
lichung dieser letzten Entwicklungsstufe der traditionellen Mühlen sollte ebenfalls Bestandteil des Nutzungskonzeptes sein.

Tab. 2: Zeitpunkt der Stilllegung der Mühlen (Daten nach Kleeberg 1964/1979 sowie Angaben der heutigen Mühlenbesitzer)

\begin{tabular}{l|l}
\hline 19. Jahrhundert & 2 \\
20. Jahrhundert bis 1960 & 13 \\
nach 1960 & 14 \\
still liegend, ohne Datum & 2 \\
in Betrieb & 4 \\
\hline unbekannt (nicht identifiziert) & 3 \\
\hline
\end{tabular}

\section{Klassifikation der Mühlen}

Im Wesentlichen lassen sich Mühlen nach ihrer Antriebsmaschine (Mühlentyp) und ihrer Arbeitsmaschine (Mühlenart) klassifizieren.

Von den untersuchten Mühlen verfügen drei noch über ein Wasserrad: die Odilienmühle (vgl. Abb. 2) und die Walkemühle in Göttingen sowie die Mühle Hartje am Rauschenwasser. Alle diese Mühlen sind stillgelegt, die ursprüngliche Einrichtung wurde entfernt.

Die noch in Betrieb befindlichen Getreidemühlen verfügen über Turbinen, doch reichen diese für den heutigen Mahlbetrieb nicht mehr aus. Die Mühle Bunte in Niedergandern produziert daher nur einen Teil des zum Mahlen benötigten Stroms. Die Turbine der Obermühle Rosdorf ist derzeit außer Betrieb, sie soll jedoch wieder genutzt werden. Die beiden Getreidemühlen sind den Motormühlen zuzuordnen.

Viele der untersuchten historischen Mühlen sind stillgelegt und zu Wohnhäusern oder Lagerräumen umgenutzt worden. Acht Besitzer, rund ein Viertel, nutzen derzeit jedoch Turbinenkraft, um Strom zu erzeugen.

Tab. 3: Antriebe der Mühlen (Daten nach Angaben der heutigen Mühlenbesitzer)

\begin{tabular}{l|l}
\hline Wasserrad & 3 \\
Turbine in Betrieb & 8 \\
Turbine defekt/außer Betrieb & 2 \\
Motor & 1 \\
Motor und Turbine & 1 \\
unbekannt & 2 \\
entfernt & 13 \\
\hline
\end{tabular}

Bei der Klassifikation nach der Mühlenart ist erkennbar, dass zwei Ausprägungen im Untersuchungsraum vorherrschten. Flächendeckend existierten Getreidemühlen, so dass sich bei Kleeberg in fast allen Gemeinden des untersuchten Gebietes 
noch Hinweise auf eine oder mehrere Getreidemühlen (meist mit den typischen Namen Ober-, Mittel- und Untermühle) finden. In beinahe der Hälfte von ihnen wird heute, da sie zu Wohnhäusern umgebaut sind, noch eine Turbine zur Stromerzeugung betrieben. Aus den Gesprächen mit den Besitzern ergab sich, dass in der Regel ein Einspeisungsvertrag mit dem Energieunternehmen E.ON AG abgeschlossen wurde, so dass der Strom eingespeist und zu einem festen Preis vergütet wird.

Eine weitere wichtige Mühlenart waren die Sägemühlen: Die Untere Rodemühle und die Mühle Uhlendorff waren z.B. reine Sägemühlen, in denen das Holz aus dem Wald der Umgebung verarbeitet wurde. Die Uhlendorff'sche Mühle wurde auf Motorkraft umgestellt und als Sägewerk weiterbetrieben. Eine ehemalige Sägemühle ist auch das Sägewerk Carstens in Rosdorf, das im Untersuchungsraum als einzige ihrer Art noch ihren ursprünglichen Zweck erfüllt, allerdings ohne gut sichtbare Hinweise auf ihre ,Mühlenvergangenheit'.

Im Stadtgebiet Göttingen sind zudem die Gebäude einer ehemaligen Walkeund einer Lohmühle (vgl. Abb. 3) erhalten. Diese lassen sich eventuell als Besonderheiten in das Nutzungskonzept integrieren. Da jedoch vom Inventar jeweils nichts mehr vorhanden ist, müssten die Mühlen erst für Besucher aufgearbeitet werden.

Tab. 4: Vorwiegende Nutzung der Mühlen (Daten nach Kleeberg 1964/1979 sowie Angaben der heutigen Mühlenbesitzer)

\begin{tabular}{l|l|l|l|l|l}
\hline $\begin{array}{l}\text { vorwiegende Nutzung (weitere in } \\
\text { Klammern) }\end{array}$ & gesamt & $\begin{array}{l}\text { heute Strom- } \\
\text { erzeugung }\end{array}$ & $\begin{array}{l}\text { davon } \\
\text { heute in } \\
\text { Betrieb }\end{array}$ & $\begin{array}{l}\text { still } \\
\text { gelegt }\end{array}$ & $\begin{array}{l}\text { unbe- } \\
\text { kannt }\end{array}$ \\
\hline Getreidemühle & 13 & 6 & $2^{*}$ & 4 & 3 \\
Getreidemühle (auch Sägemühle) & 1 & 1 & 0 & 0 & 0 \\
Getreidemühle und Ölschlag & 1 & 0 & 0 & 1 & 0 \\
Sägemühle & 3 & 0 & 0 & 3 & 0 \\
Sägemühle (auch Getreidemühle) & 1 & 0 & $1^{*}$ & 1 & 0 \\
Walkemühle & 1 & 0 & 0 & 0 & $1^{* *}$ \\
Lohmühle & 1 & 0 & 0 & 1 & 0 \\
unbekannt & 10 & 4 & 0 & 6 & 0 \\
Gesamt & 31 & 11 & 3 & 16 & 4 \\
\hline
\end{tabular}

* $=$ mit Motorkraft

** = Die Walkemühle dient nicht mehr ihrem ursprünglichen Zweck. Sie ist zwar stillgelegt, jedoch befindet sich daran ein Mühlrad, das sich (wieder) dreht. Es war jedoch nicht klar, ob dies nur "zur Schau“ ist oder damit auch ein Generator angetrieben wird. Daher die Einteilung „unbekannt“. 


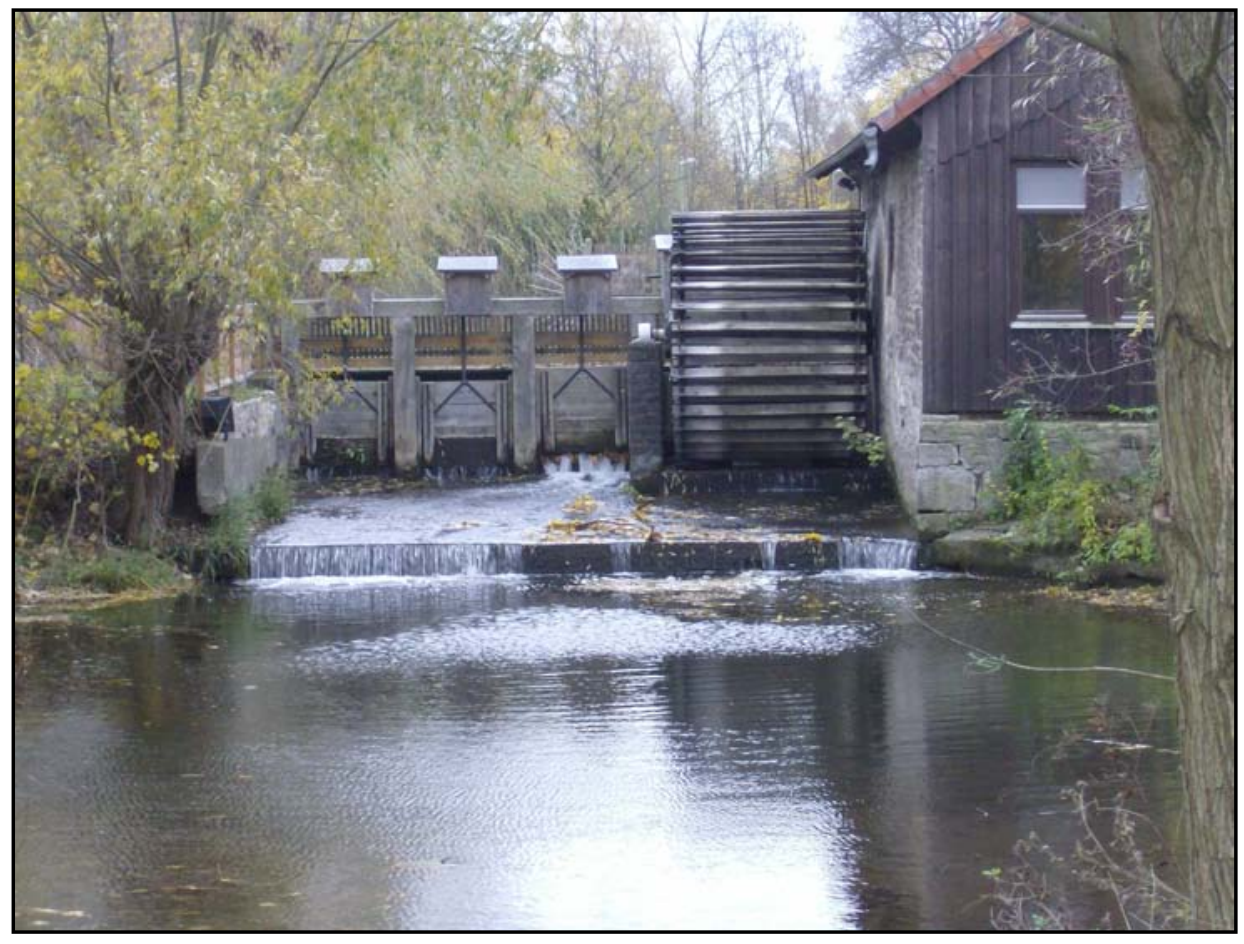

Abb. 3: Walkemühle in Göttingen (Foto: Wieland)

\section{Das Konzept des Mühlenwanderweges}

Das Konzept eines Mühlenwanderweges sollte versuchen, ein möglichst vollständiges Bild der ehemaligen Mühlen zu liefern. In Bezug auf die Mühlenarten sollte daher angestrebt werden, neben den Getreidemühlen auch mindestens eine der Sägemühlen sowie die Walke- und Lohmühle zu integrieren. Selbst wenn die ehemaligen Nutzungen kaum noch im Detail erkennbar sind, so sind sie im Rahmen des Konzeptes von besonderem Interesse, da sie andeuten, wie groß die Vielfalt der Mühlenarten (teilweise bis ins 20. Jahrhundert hinein) einmal war.

Die Mühlen, deren Antrieb nicht rückgebaut wurde, sind im Untersuchungsraum mehrheitlich mit Turbinen ausgestattet. Aus diesem Grund gewinnen die Mühlen mit Wasserrädern an Bedeutung, da die Touristen im Zusammenhang mit dem Begriff Wassermühle ein Wasserrad erwarten. Die Tatsache, dass zahlreiche Mühlen zuletzt mit einer Turbine ausgestattet waren, sollte als Teil des technischen Fortschritts angesprochen werden.

Angesichts der beachtlichen Zahl an Mühlenbesitzern, die mit ihren Turbinen Generatoren zur Stromerzeugung antreiben, könnte es zudem interessant sein, 
diese Weiternutzung zu berücksichtigen. Neben der Entwicklung des Mühlenwesens bis zum Mühlensterben könnte so eine ganz neue und angesichts der Diskussion um erneuerbare Energien aktuelle Variante der Wasserkraftnutzung aufgezeigt werden. Auf diese Weise könnte die Antriebstechnik der Mühlen als nicht veraltet, sondern als zeitlos brilliante Nutzung der Wasserkraft herausgestellt werden.

Die Auswahl der Mühlen für den Mühlenwanderweg erfolgt anhand zweier Kriterien. Das erste Kriterium bildet die durch den Erfassungsbogen festgehaltene Attraktivität der Mühle, die sich aus der Gesamtpunktzahl des Objektes ergibt. Das zweite Kriterium stellt der Standort einer geeigneten Mühle im Vergleich zu anderen gut bewerteten Mühlen dar. Dieses Kriterium ist mit dem ersten gleichgestellt, da Mühlenstandorte so gelegen sein müssen, dass sie sich zu einem ,Weg', einem Gesamtkonzept, zusammenfassen lassen.

Um die Wertigkeit der Punktzahlen einer Mühle festzulegen, wird ein Mindestwert festgelegt, den eine Mühle erreichen muss, um für den Mühlenwanderweg in Betracht zu kommen. Der Wert der Idealmühle beträgt 21,5 Punkte. Zur Vergleichbarkeit wird ihr die Wertung für eine Durchschnittsmühle, die sich aus den kumulierten Einzelwerten aller Mühlen ergibt, gegenübergestellt. Hierbei werden die Mühlen mit negativem Gesamtwert und solche, deren Mühlengebäude verschwunden bzw. nicht identifizierbar war, ausgeschlossen. Ehemalige Mühlenstandorte halten zwar die Erinnerung an einen Mühlenbetrieb wach, können jedoch innerhalb eines touristischen Konzeptes (mit Betonung auf der Nutzung) nicht eingesetzt werden. Der Gesamtwert der Durchschnittsmühle beträgt 8,6. Ausgehend von diesen beiden Werten wird festgelegt, dass eine Mühle mindestens die Hälfte der durch die Idealmühle definierten Höchstpunktzahl, abgerundet 10,5 Punkte, erreichen muss, um den Anforderungen einer touristischen Nutzung zu genügen.

Mühlen, deren Wert in Anlehnung an die Durchschnittsmühle bei mindestens 8 Punkten liegt, werden für eine Aufnahme in das Konzept in Betracht gezogen, sofern sie sich aus bestimmten Gründen (etwa die besondere Anschaulichkeit bestimmter Gegenstände oder Prozesse) trotz ihres niedrigen Erfassungswertes für die Mühlenwanderung eignen.

Auf Grund ihrer Gesamtpunktzahl im Erfassungsbogen werden folgende historische Mühlen zur Aufnahme in ein Nutzungskonzept vorgeschlagen: Leinemühle Bunte, Haellenmühle, Mühle Gut Besenhausen, Reckershauser Mühle, Leinemühle Stockhausen, Mühle Niedernjesa, Obermühle Rosdorf, Untere Mühle Rosdorf, Walkemühle Göttingen, Odilienmühle Göttingen, Wassermühle Hartje, Untere Rodemühle, Mühle Sonne.

Um ein dichteres touristisches Netz von Mühlen aufzubauen, sollte gegebenenfalls in Betracht gezogen werden, das Angebot bei besonderen Bemühungen zu erweitern, sei es bei aufwändiger Inwertsetzung oder durch die Schaffung von Angeboten, die von der authentischen Bausubstanz der historischen Mühlen unabhängig sind. 
Das Elektrizitätswerk Northeim ist ebenfalls erfasst worden, um eventuell in einem Konzept präsentiert zu werden, das die Entwicklung der Turbinentechnik thematisiert. Das Elektrizitätswerk hat die erforderliche Punktzahl erreicht, auch wenn es keine historische Wassermühle ist. Außerdem wird vorgeschlagen, die Rhumemühle Northeim in ein Nutzungskonzept zu integrieren, auch wenn sie bei der quantitativen Auswertung schlecht abgeschnitten hat. Die Abwertung ist vor allem auf Grund des Umfeldes erfolgt, die Mühle könnte dennoch auf Grund ihres beachtlichen Gebäudes und der sichtbaren Wehranlage Berücksichtigung finden.

Die geeigneten Mühlen sind durch den Leineradweg miteinander verbunden, die Abstände untereinander sind gering genug, um mit dem Fahrrad überwunden zu werden (vgl. Abb. 1).

\section{Zum Inhalt des Konzeptes}

Das Rahmenthema des Konzeptes sind die historischen Mühlen des Untersuchungsraumes „Mühlen in Göttingen und Umgebung“. Bei der Setzung thematischer Schwerpunkte sollten verschiedene Feststellungen berücksichtigt werden, die sich aus der Bestandsaufnahme ergeben. Auf die ausgewählten Mühlen im Untersuchungsgebiet trifft zu:

1) Die Mühlen weisen mehrere Mühlenarten auf, nämlich je eine Walke- und eine Lohmühle sowie drei Sägemühlen; den Hauptteil machen die Getreidemühlen aus, von denen zwei noch in Betrieb sind. Diese Mühlen bieten sich für Besichtigungen an.

2) Derzeit weisen drei Mühlen ein Wasserrad auf, zehn verfügen über eine Turbine und zwei mahlen mit Motorkraft. Die Mühlen sind in der Mehrzahl nicht ohne weiteres für Touristen erkennbar, sie bedürfen der ,Sichtbarmachung' im Rahmen einer touristischen Inszenierung. Dies kann in der Mehrzahl der ausgewählten Mühlen jedoch nicht über die Rekonstruktion eines Wasserrades erfolgen, weil dadurch die Turbinen nicht mehr arbeiten könnten. Infolgedessen müssen die Mühlen auf anderem Wege für die Besucher als Mühlen erkennbar werden, z.B. durch Hinweise oder einen kreativen Ersatz der mühlentypischen Bauteile. Bei den Motormühlen erübrigt sich ein Ersatz, sie sind so zu präsentieren, wie sie sind.

3) Im Fall der Mühlen, die auf Turbinenantrieb umgestellt worden sind, bietet sich eine Thematisierung der Turbinentechnik und der Stromerzeugung als zeitlose Form der Energiegewinnung an. Hiermit könnte die Entwicklungslinie von den historischen Mühlen bis in unsere Zeit aufgezeigt werden, insbesondere vor dem Hintergrund der aktuellen Debatte um $\mathrm{CO}_{2^{-}}$ Emissionen bei der Stromerzeugung und die Förderung regenerativer Energien. 
4) Von den ausgewählten Mühlen wurden eine im 13., vier im 14. und eine weitere im 15. Jahrhundert erstmals erwähnt. Bei diesen mittelalterlichen Mühlen kann insbesondere auf die Bedeutung der Klostermühlen und des herrschaftlichen Mühlenbaus eingegangen werden.

5) Neun Mühlen wurden im 20. Jahrhundert bis 1960, drei danach still gelegt; zwei Getreidemühlen und das später gebaute Elektrizitätswerk sind in Betrieb. Die Stilllegung der anderen Mühlen könnte in einem thematischen Schwerpunkt zum Mühlensterben behandelt werden.

\section{Thematische Schwerpunkte des Mühlenwanderweges}

Bei der Gestaltung des inhaltlichen Rahmens der Mühlenroute ist anzumerken, dass der Mühlenbestand des Untersuchungsraumes auf Grund häufiger Umnutzungen und Entfernen der mühlentypischen Merkmale der touristischen Inszenierung gewisse Grenzen setzt.

So ist es nur bedingt möglich, der Erwartungshaltung der Touristen nach einem laufenden „Wasserrad“ nachzukommen. Die meisten Mühlen verfügen nicht mehr über diesen Antrieb, denn schon vor gut 150 Jahren waren Wasserräder nicht mehr der letzte Stand der Technik, und viele der damals noch in Betrieb befindlichen Mühlen wurden umgerüstet. Im 19. Jahrhundert begann außerdem der Trend zum Bau zunehmend größerer Wassermühlen.

Neben der größeren Leistung brachten die Turbinen mit sich, dass sie den Wassermühlen ihr nach außen erkennbares Zeichen nahmen. Für die damaligen Müller war das nicht problematisch, sie hatten ihre technische Anlage an die Bedingungen des sich verschärfenden Wettbewerbs angepasst. Für den Untersuchungsraum ist es als ein Anzeichen für das Schritthalten mit der wirtschaftlichen Entwicklung im 19. und 20. Jahrhundert zu sehen. Die Frage im touristischen Verwertungszusammenhang ist, wie man mit diesem ,Mangel' umgeht. Eine Rückkehr von Mühlenrädern zu touristischen Zwecken dürfte zwar seine Wirkung auf Besucher nicht verfehlen, doch ist es fraglich, ob eine Verfälschung der betreffenden Mühle berechtigt ist, denn mit dem Einbau einer Turbine ist die Nutzung eines Wasserrades zumindest entwicklungsgeschichtlich vorbei. Aus diesem Grund sollte der inhaltliche Schwerpunkt einer Mühlenroute auf der Tatsache liegen, dass die meisten Mühlen verschwunden sind und die wenigen heute noch vorhandenen meist nicht mehr als den Namen, das Gebäude und den Turbinenantrieb aufweisen. Mit diesem Sachverhalt sollte gegenüber den Besuchern ganz offen umgegangen werden, ist er doch ein Zeugnis für den vollzogenen Strukturwandel im Untersuchungsraum. Gleiches gilt für das Themenfeld der Turbinentechnik im Kontext der Stromerzeugung, was eine Zukunftsperspektive für die Mühlen aufzeigt.

Auch sollte auf die Ursprünge bestimmter Mühlen, vor allem auf Klostermühlen und den herrschaftlichen Mühlenbau, eingegangen werden. Die Vielfalt der 
Mühlenarten kann herausgestellt werden, wenn gleichzeitig betont wird, dass sie heute nicht mehr vorhanden ist. In Zusammenhang mit der Vergangenheit kann auch auf die soziale Stellung des Müllers eingegangen werden. Gerade die wenigen Mühlen, die über ein Wasserrad verfügen und damit am ,altmodischsten' sind, eignen sich für diese Thematik. Anhand der Wasserräder könnten auch unterschiedliche Beaufschlagungstypen dargestellt werden.

\section{Ausblick}

Ein wichtiger Bestandteil der Mühlenthematik konnte hier nicht berücksichtigt werden: die kulturelle Bedeutung der Mühlen, die sich in Literatur, Kunst und der Sprache widerspiegelt (vgl. Büllesbach 1994). Gerade dieser Themenkomplex bietet viele Ansätze für eine touristische Inwertsetzung historischer Mühlen.

Zudem hat sich die bis ins Mittelalter zurückreichende Mythenbildung um Mühlen bis heute erhalten, die sich besonders gut für erlebnisorientierte Konzepte eignen dürfte. Auch in der Kunst gibt es zahllose Beispiele von gemalten Mühlen (vgl. Mager et al. 1989), an die angeknüpft werden könnte. Zur kulturellen Bedeutung der Mühlen wäre für den Untersuchungsraum eine Recherche sinnvoll, um den skizzierten Mühlenwanderweg um diesen interessanten Aspekt bereichern zu können. 


\section{Literatur und Quellen}

Arbeitsgruppe Mühlenstraße in der Mühlenvereinigung Niedersachsen-Bremen e.V. (1995): Die Niedersächsische Mühlenstraße. Reisewege zu 73 Mühlen zwischen Nordsee, Elbe und Weser. [Faltblatt]

Bardua, S. (2006): Vergessene Denkmale einer modernen Kulturlandschaft? Industriemühlen in Mecklenburg, Hamburg und Schleswig-Holstein. In: Nagel, F. N. (Hrsg.): Türme, Schornsteine, Industrie-Mühlen und Land-Art. Books on Demand Verlag, Norderstedt. S. 57-96.

Büllesbach, C. (1994): Mühlendarstellungen. Form und Gleichnis. Konstruktion und Funktion. Die Trennung von Innen und Außen. o.A.

Gawehns, H. C. (1999): Denkmalschutz und geschichtliche Architektur. Inaugural-Dissertation. Nürnberg.

Eidevik, E. (2007): Die Mühlen in Südniedersachsen - Bestand und Zukunft. Hausarbeit im Rahmen der Ersten Staatsprüfung für das Lehramt an Gymnasein. Universität Göttingen.

Heinze, T. (1999): Konzeptionelle und marketingstrategische Überlegungen zum (regionalen) Kulturtourismus. In: ders. (Hrsg.): Kulturtourismus. Grundlagen, Trends und Fallstudien. Oldenbourg, München/Wien. S. 1-16.

Jahn, E. (2002): Historische Mühlen als öffentliche Aufgabe und Konzept zu ihrer Erhaltung, Sanierung und Nutzung. In: Schmidt, L. \& Varchmin, J. (Hrsg.): Maschine, Denkmal, Landmarke: Historische Mühlen - Konflikt um eine Denkmalgattung. Cottbus.

Kleeberg, W. u. Vereinigung zur Erhaltung von Wind- und Wassermühlen in Niedersachsen e.V. (1964): Niedersächsische Mühlengeschichte. Bösmann Verlag, Detmold.

Kleeberg, W. u. Vereinigung zur Erhaltung von Wind- und Wassermühlen in Niedersachsen e.V. (1979): Niedersächsische Mühlengeschichte. Bösmann Verlag, Detmold.

Löbert, H. W. (2001): Vorwort. In: Mühlenförderverein Lüneburg e.V. u. Thiemann, H. (Hrsg.): Alte Mühlen - neu entdeckt: aus der Niedersächsischen Mühlenstraße. Christians Verlag, Hamburg.

Mager, J., Meißner, G. u. Orf, W. (1989): Die Kulturgeschichte der Mühlen. Wasmuth Verlag, Tübingen.

Möhlenkamp, A. (1998): Die Mühle in Märchen und Recht. In: Mühlenförderverein Lüneburg e.V. u. Thiemann, H. (Hrsg.) (2001): Alte Mühlen - neu entdeckt: aus der Niedersächsischen Mühlenstraße. Christians Verlag, Hamburg. 
Möller, H.-H. u. Niedersächsisches Landesverwaltungsamt - Institut für Denkmalpflege - (1984): Was ist ein Kulturdenkmal? Arbeitshefte zur Denkmalpflege in Niedersachsen 2. Niemeyer Verlag, Hameln.

Mühlenförderverein Lüneburg e.V. (Hrsg.) u. Thiemann, H. (2001): Alte Mühlen - neu entdeckt: aus der Niedersächsischen Mühlenstraße. Christians Verlag, Hamburg.

Schlender, T. (1988): Alte Mühlen in Mythen, Märchen und Erzählungen. Droemer/Knaur, München.

Schmidt, L. (2002): Vorwort. In: Schmidt, L. \& Varchmin, J. (Hrsg.): Maschine, Denkmal, Landmarke: Historische Mühlen - Konflikt um eine Denkmalgattung. Cottbus.

Schröder, A. (2003): Industrietourismus. In: Becker, C., Hopfinger, H. u. Steinecke, A. (Hrsg.): Geographie der Freizeit und des Tourismus: Bilanz und Ausblick. Oldenbourg, München/Wien. S. 213-224.

Steinecke, A. (1999): Perspektiven des Kulturtourismus: Wettbewerbsdruck Profilierung - Inszenierung. In: Heinze, T. (Hrsg.): Kulturtourismus. Grundlagen, Trends und Fallstudien. Oldenbourg, München/Wien. S. 17-51.

Storch, J. u. Paschke, R. (2002): Bestimmung von Denkmalwerten. In: Schmidt, L. u. Varchmin, J. (Hrsg.): Maschine, Denkmal, Landmarke: Historische Mühlen - Konflikt um eine Denkmalgattung. Cottbus.

Varchmin, J. (2002): Konfliktfeld Mühle. In: Schmidt, L. u. Varchmin, J. (Hrsg.): Maschine, Denkmal, Landmarke: Historische Mühlen - Konflikt um eine Denkmalgattung. Cottbus.

Wagenbreth, O., Düntzsch, H., Tschiersch, R. u. Wächtler, E. (1994): Mühlen. Geschichte der Getreidemühlen. Technische Denkmale in Mittel- und Ostdeutschland. Spektrum Akademischer Verlag, Leipzig/Stuttgart.

Woelk, S. (2001): Müller und Mühlen sozialgeschichtlich. In: Mühlenförderverein Lüneburg e.V. u. Thiemann, H. (Hrsg.): Alte Mühlen - neu entdeckt: aus der Niedersächsischen Mühlenstraße. Christians Verlag, Hamburg.

Wolf, A. (2005): Erfolgsfaktoren industriehistorischer Einrichtungen. Eine Untersuchung zu Erfolgsfaktoren unterschiedlicher Angebotstypen und ausgewählter Einrichtungen in Großbritannien und Deutschland (=Paderborner Geographische Studien zu Tourismusforschung und Destinationsmanagement, Bd. 18). Selbstverlag der Universität Paderborn. 
Landschaftswandel und Wahrnehmung Das Beispiel Offshore-Windkraft

Kira Gee 


\begin{abstract}
Offshore wind is representative for a new paradigm of use that is currently emerging in the German North Sea. Various policy levels converge to promote the use of the sea as an energy space; added to this are a range of push and pull factors driving wind energy offshore. Spatially, the sea is now treated much like the mainland, with terrestrial land use planning approaches largely taken over unchanged and large-scale fixed infrastructure becoming commonplace. These developments collide with views of the sea as a natural 'unspoilt' environment and the idea of an untouched seascape. There is a growing imbalance between traditional-romantic views of the sea and the prospect of the sea becoming increasingly 'built up'. Despite this divergence, survey results from the West coast of Schleswig-Holstein also show potential trade-offs, largely because renewable offshore energies are considered a less than ideal but necessary energy option for the future.
\end{abstract}

\title{
Einführung
}

Über die Jahrhunderte hatten viele Kulturen ein eher zwiespältiges Verhältnis zum Meer. Rationale Aspekte der Nutzung und der mögliche Gewinn aus einer Nutzung waren stets gekoppelt an das Bewusstsein der Unberechenbarkeit des Meeres und seiner eher emotionalen Wahrnehmung als Ort der Gefahr. Diese Zwiespältigkeit der Wahrnehmung ist bis heute erhalten geblieben. Das Meer wirkt kalt, abweisend und gefährlich und ist gleichzeitig verbunden mit Sommer, Sonne und Strand; der Ozean ist Grenze, unerforschte Tiefe und Naturraum und steht gleichzeitig für einen Kulturraum im Sinne von Jahrhunderte alten Handelstraditionen. Auch in Deutschland haben sich viele unterschiedliche Zugänge zum Meer entwickelt. Kontemplativ-ästhetische und wissenschaftliche Zugänge zu Meer und Küste entstanden erst vergleichsweise spät im 19. Jahrhundert im Kontext der beginnenden touristischen Erschließung der Küste (vgl. Fischer et al. 2007). Heute fällt die Gleichzeitigkeit verschiedener Konstrukte mit unterschiedlichen Interessen und der unterschiedlichen Durchsetzungsmacht der beteiligten Akteure zusammen - ausschlaggebend für die Entstehung neuer Nutzungsparadigmen und Nutzungskonflikte im Meer.

Dieser Beitrag greift die Inszenierung des Meeres als Energieraum als eine spezielle, da aktuelle und kontroverse Inszenierung heraus. Getragen wird diese vor allem von der Offshore-Windkraft, die durch ihr relativ schnelles Aufkommen und den hohen Raumbedarf ganz neue Anforderungen an den Meeresraum stellt. Diesen Anforderungen nachzukommen bedeutet einen grundlegenden Wandel in der Meeresnutzung und vor allem auch in der sichtbaren Meereslandschaft. Dies 
wiederum wirft Fragen nach den gegenwärtigen Interpretationen und möglichen zukünftigen Vorstellungen von Meeresraum und -landschaft auf. Welche anderen Konstrukte vom Meer sind mit der Offshore-Windkraft kompatibel, welche sind es nicht? Wird die Vision vom Meer als Energieraum von der Gesellschaft mitgetragen, oder gibt es Gründe, sich dagegen auszusprechen?

Hier soll der These nachgegangen werden, dass es im Zuge des gegenwärtigen Nutzungswandels zu einer Entkopplung zwischen der Offshore-Windkraftentwicklung im Meer - getrieben von gezielten Interessen der handelnden Institutionen und Organisationen - und den oftmals latenten Vorstellungen vom Meer in den Augen der lokalen Betrachter kommt. Real wahrnehmbare Veränderungen im Meeresraum beginnen, die existierenden Vorstellungen vom Meer zu überholen; es kommt zu einem Ungleichgewicht zwischen neuen und bereits existierenden Meeresbildern. Andererseits wird postuliert, dass gerade erneuerbare Energien eine Brücke zwischen verschiedenen und auch konträren Meeresvorstellungen schlagen können. Grund dafür ist, dass sie sowohl akzeptanzmindernde als auch -fördernde Aspekte in sich tragen und sich schon alleine aufgrund ihrer moralischen Komponente einer einfachen Kategorisierung als ,erwünscht' oder ,unerwünscht' entziehen (vgl. Byzio et al. 2005). Neben diesem akuten Dilemma für den Meeresbetrachter führt dies auch zu neuen Möglichkeiten der Abwägung und Kompromissbildung. Offshore-Wind ist vielleicht nicht die präferierte Lösung, aus bestimmten Gründen aber dennoch eine akzeptable Variante für den zukünftigen Meeresraum.

Im Folgenden werden zunächst aktuelle Entwicklungstrends und die treibenden Rahmenbedingungen für den Nutzungswandel im Meer skizziert. Anschließend dienen die Sichtweisen der Bewohner der schleswig-holsteinischen Westküste auf das Meer und die Offshore-Windkraft als Beispiel für lokale Perspektiven. Grundlage ist eine schriftliche Befragung, die Ende 2005 in ausgewählten Gemeinden in Nordfriesland und Dithmarschen durchgeführt wurde. Insgesamt 387 Anwohner wurden in offenen und geschlossenen Fragen zu ihrer Sichtweise der Küsten- und Meereslandschaft und der Offshore-Windkraft befragt. Die Auswahl der Befragten erfolgte randomisiert in einem zweistufigen Auswahlverfahren, doch sind die Ergebnisse aufgrund des kleinen Samples nur begrenzt repräsentativ (vgl. Gee 2009).

\section{Aktuelle Nutzungstrends und Offshore-Windkraft: Ein Pa- radigmenwechsel}

\section{Nutzungswandel im Meer und neues Raumverständnis}

In den europäischen Meeren zeichnet sich seit einigen Jahren eine Art Aufbruchsstimmung ab. Eingebettet in die Frage, wie sich eine nachhaltige Nutzung gestalten lässt, erfahren die Meere eine deutliche Aufwertung als Wirtschafts-, Trans- 
port- und Industrieraum. Unterschiedliche Entwicklungen treffen aufeinander: Neue Nutzungskonzepte entstehen im Offshore-Bereich; das Nutzungsspektrum insgesamt weitet sich aus, gleichzeitig werden diverse etablierte Sektoren wie z.B. der Seeverkehr deutlich intensiver (vgl. Gee et al. 2006) (Abb. 1). Getrieben werden diese Entwicklungen von globalen Trends wie der wachsenden Internationalisierung des Handels, dem steigenden Wettbewerb zwischen Wirtschaftsregionen oder neuen geopolitischen Interessen (z.B. in der Arktis). Parallel dazu führen der Klimadiskurs und die Frage nach einer energiepolitischen Neuorientierung zu ungewohnten Konvergenzen zwischen Umweltschutz und Wirtschaft. Deutlich werden diese Tendenzen z.B. in der aktuellen Meerespolitik der EU, die einerseits das Prinzip der Nachhaltigkeit betont, deren Ziel jedoch vor allem die Stärkung der europäischen Wettbewerbsfähigkeit ist (vgl. Kommission der Europäischen Gemeinschaften 2007). Sichtbar werden sie aber auch in Bestrebungen, den Ausbau der erneuerbaren Energien generell - rhetorisch zumindest - an zu erwartende ökonomische Gewinne zu knüpfen („,A green new deal“) ${ }^{1}$.

Auch in Deutschland wird die verstärkte Nutzung und die Gewinnung erneuerbarer Energie im Meer als Chance begriffen (vgl. BMVBS 20092; BMU 2007; Ministerium für Wissenschaft, Wirtschaft und Verkehr des Landes SchleswigHolstein 2007). Die Bundesregierung beispielsweise argumentiert, dass die nationalen Klimaziele ohne einen zügigen Ausbau der Offshore-Windkraft nicht erreicht werden können (vgl. BMU 2002); gleichzeitig verspricht man sich davon nationale und regionale Beschäftigungseffekte (vgl. BMWi 2008).

\footnotetext{
${ }^{1}$ Investitionen in Höhe von 24 Milliarden Euro in einen Green New Deal könnten nach Berechnungen der Unternehmensberatung A.T. Kearney langfristig 500.000 neue Arbeitsplätze in Deutschland schaffen. Aus: http://www.wiwo.de/unternehmer-maerkte/green-deal-koennte-500-000arbeitsplaetze-schaffen-384199/, Zugriff am 29.6.09

2 BMVBS unter http://www.bmvbs.de/Verkehr/Wasser-,1466.912520/Maritime-Wirtschaft.htm, Zugang am 22.5.09
} 


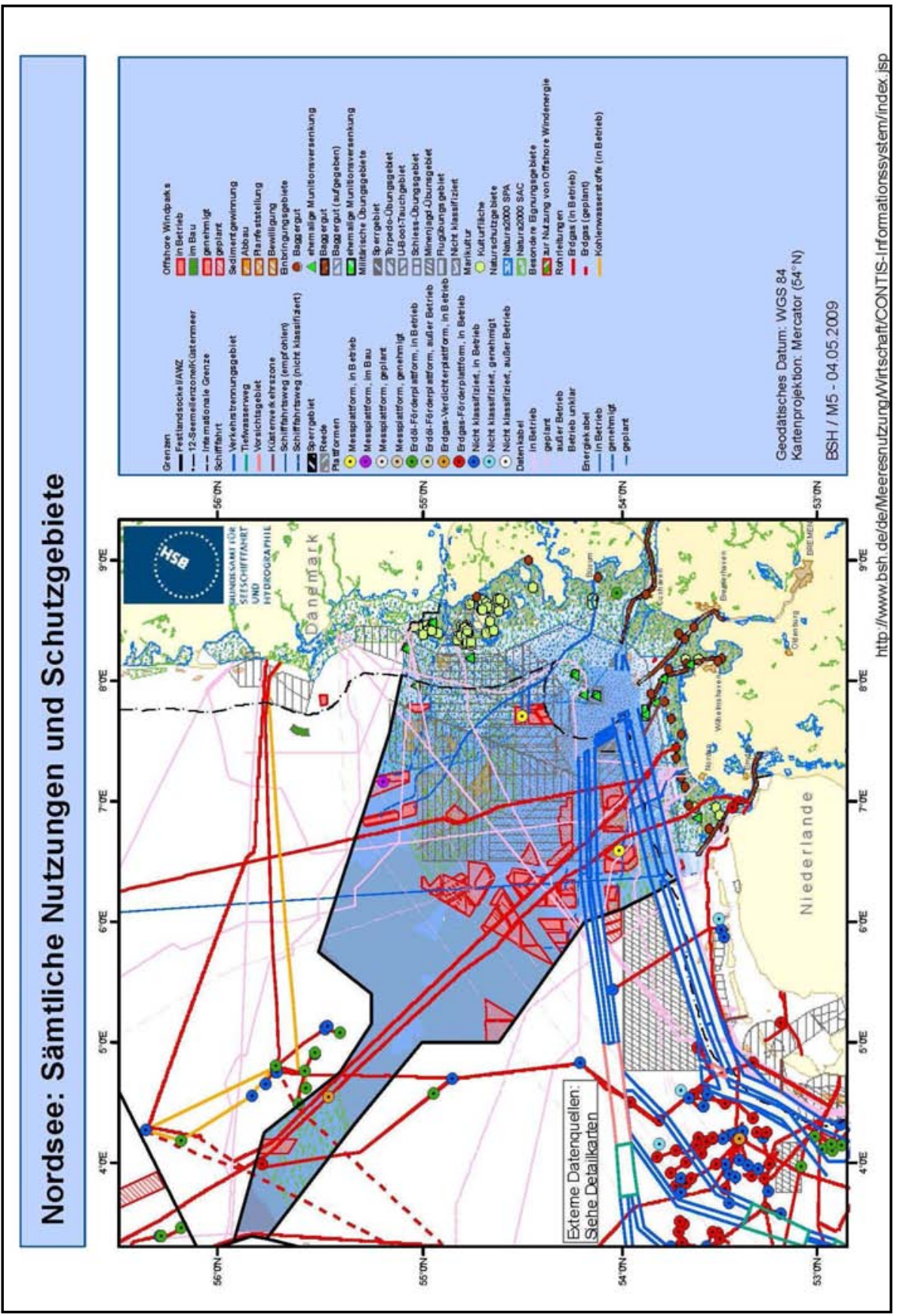

Abb. 1: Sämtliche Nutzungen und Schutzgebiete in der deutschen Nordsee,

Stand 04.05.2009 (Quelle: BSH 2009, www.bsh.de) 
Nach anfänglicher Skepsis hält inzwischen auch die Windbranche die OffshoreNutzung für eine teure, aber gangbare Option. Pull-Faktoren sind das Versprechen einer deutlich höheren Energieausbeute, feste Rahmenbedingungen wie das Erneuerbare-Energien-Gesetz, das eine Einspeisevergütung für Offshore-Strom über einen Zeitraum von 20 Jahren verspricht ${ }^{3}$, sowie die Ausweisung von Eignungsflächen für die Offshore-Windkraft in der Ausschließlichen Wirtschaftszone (AWZ) (vgl. BSH 2009). Zu den Push-Faktoren zählen die wachsenden Landnutzungskonflikte in ländlichen Räumen und die eskalierende Debatte um die ,Verspargelung' der Landschaft, in der ästhetische und ökologische Bedenken mit Fragen der Kosten- und Nutzenverteilung sowie Fragen der Macht und Entscheidungsfindung zusammentreffen. Derzeit befinden sich mehr als 50 OffshoreProjekte in Planung, 18 davon sind genehmigt (vgl. Abb. 2).

Als neue, großflächige Infrastruktur stehen Offshore-Windparks für einen grundlegenden Wandel in der Art der Meeresnutzung. Neu ist hier weniger das Prinzip der fixen Strukturen im Meer als der außergewöhnlich hohe Flächenbedarf gekoppelt an die Tatsache, dass nur wenige Akteure den Ausbau der OffshoreWindkraft prinzipiell hinterfragen (vgl. Licht-Eggert et al. 2008). Angesichts der gegenwärtigen Rahmenbedingungen spricht daher viel für einen weiteren Ausbau des Sektors.

Diese neue Art der Meeresnutzung ist exemplarisch für eine allgemeine Verschiebung in der Wahrnehmung des Meeresraums. Technologische Entwicklungen haben zu einer Überwindung der Grenzen zwischen Land und Meer geführt; vieles ist machbar geworden, was vor Jahren noch undenkbar erschien. Ein neuer Umgang mit der Ressource Meer ist möglich, der sich von den bisher eher flüchtigen Formen der Nutzung unterscheidet. Vieles hängt mit einer neuen Art der räumlichen Inanspruchnahme zusammen. Statt sich wie die Fischerei nach den vorhandenen Ressourcen zu richten, oder wie die Schifffahrt eine gewisse temporäre Flexibilität zu besitzen, entsteht mit der Offshore-Windkraft eine Nutzung, die unverrückbar im Meer steht, die alle drei Raumdimensionen im Meer vom Meeresboden bis hin zur Meeresoberfläche beansprucht und die zudem andere Nutzungsformen ausgrenzt. ${ }^{4}$

\footnotetext{
${ }^{3}$ Erneuerbare-Energien-Gesetz (EEG) der Bundesregierung vom 25. Oktober 2008, BGBl. I S. 2074, zuletzt geändert durch Art. 5 G. v. 28.3.2009 I 643.

${ }^{4}$ Z.B. Schifffahrt und Fischerei; die Begründung hierfür sind Sicherheitsbedenken.
} 


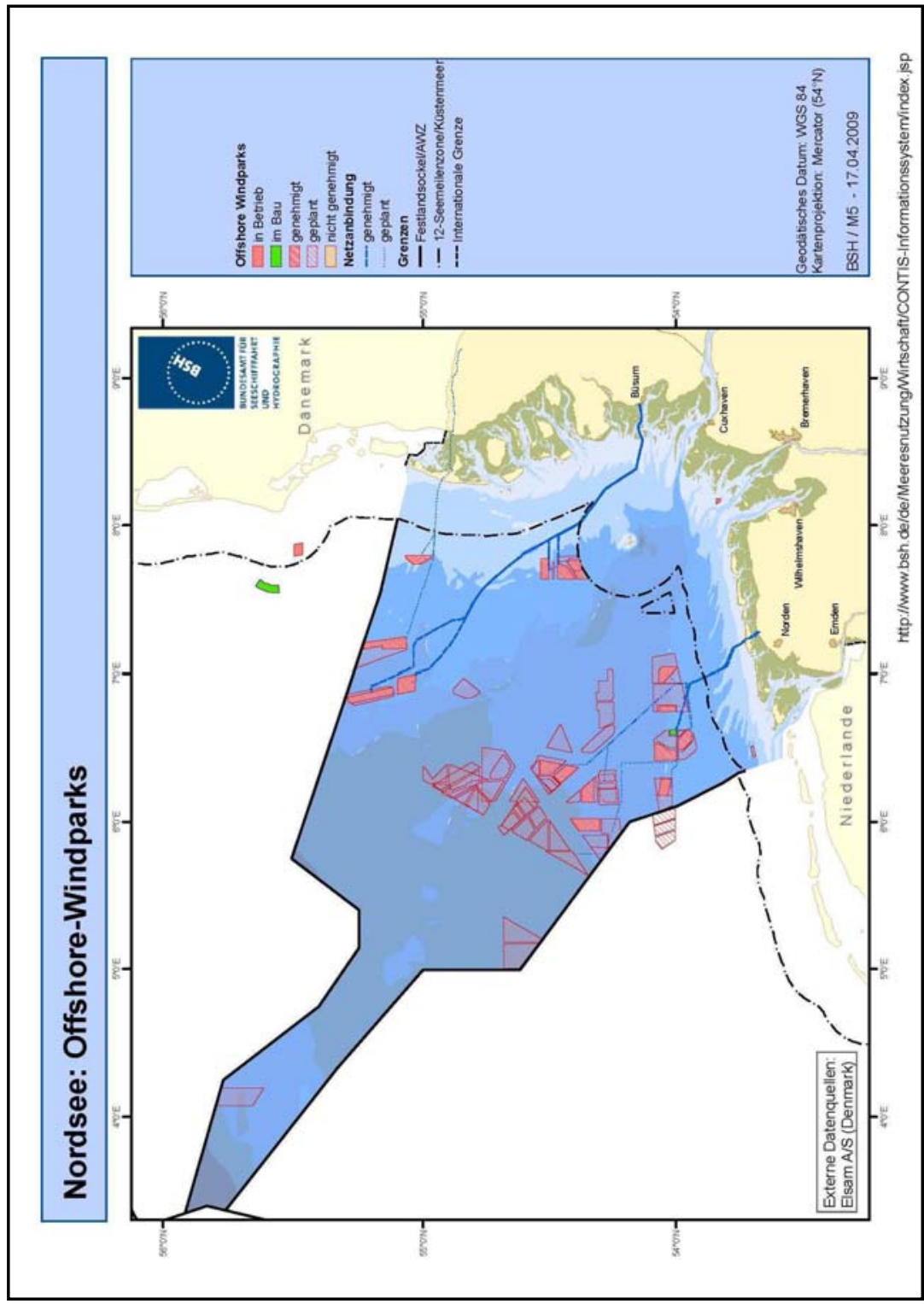

Abb. 2: Geplante und genehmigte Offshore-Windparks in der deutschen Nordsee, Stand: 17.04.2009 (Quelle: BSH, CONTIS Informationssystem 2009, www.bsh.de/de/Meeresnutzung/ Wirtschaft/CONTIS-Informationssystem/) 
Die Offshore-Windkraft ist die Ausprägung eines Raumverständnisses, das das Meer als Verlängerung des Festlandes betrachtet und ein terrestrisches Nutzungsparadigma mehr oder weniger unverändert dorthin überträgt. Neben der Ölförderung, die Fläche eher punktuell in Form von Förderplattformen in Anspruch nimmt, ist die Offshore-Windkraft die erste Nutzung, die einer derart ausgedehnten baulichen Überprägung des vormals ,leeren' Meeres gleichkommt. Dies macht deutlich, dass sich das Meer vom vermeintlich unnahbaren, trotz allem unberechenbaren Raum zu einem domestizierten Nutzungsraum entwickelt hat - eine Tendenz, die von den treibenden Akteuren in keiner Weise in Frage gestellt, sondern im Sinne der erwähnten Aufbruchsstimmung sogar explizit begrüßt wird.

Einhergehend mit diesem Wandel ist aber auch ein Verständnis von ,Meeresraum ' im Sinne einer Raumordnung, definiert hier als normativer Ansatz zur Entwicklung, Ordnung und Sicherung des Raums. Die beginnende Meeresraumordnung (vgl. Douvere \& Ehler 2009, Ministerium für Arbeit, Bau und Landesentwicklung Mecklenburg-Vorpommern 2005) ist eine direkte Übertragung terrestrischer Raumordnungsparadigmen und ihrer Mechanismen als Antwort auf die steigende Raumknappheit im Meer. Diese direkte Übertragung wird durchaus kritisch gesehen (vgl. Bruns \& Gee 2009) und ist auch deshalb bedenklich, da nicht geklärt ist, welche Bedeutung dem Begriff ,Meeresraum 'im normativen Sinn denn eigentlich zugewiesen wird (vgl. Blotevogel 2005)5. Bisher konzentriert sich die Meeresraumordnung primär auf den physischen und gesellschaftlichen Raum; die Leitvorstellung der Raumnutzung ist neben der Sicherung der natürlichen Lebensgrundlagen eine möglichst objektive Zuteilung von Nutzfläche. Subjektbezogene Raumkonzepte wie z.B. das Meer als symbolischer Raum, das Meer als Landschaft oder auch systemtheoretische Perspektiven werden derzeit für den Meeresraum kaum diskutiert. Dass dies zu einer limitierten Sichtweise auf den Meeresraum führt, macht das folgende Kapitel deutlich. ,Weiche‘ Steuerungselemente wie das integrierte Küstenzonenmanagement (IKZM) oder partizipative Formen der Raumplanung, die dieser Limitierung entgegenwirken könnten, kommen bisher nicht zur Anwendung.

\section{Das Meer - mehr als nur Nutzungsfläche?}

Die eher abstrakte planungsorientierte und utilitaristische Sichtweise des Meeres kollidiert mit einer gänzlich anderen Belegung des Meeres im Kontext seiner Nutzung als Kulisse, als Naturraum und als konstituierender Bestandteil des Lebensraums Küste. Insbesondere in letzterem Fall ist das Meer keine Fläche, sondern eindeutig eine Landschaft, ein Punkt der Verortung, Sinnstiftung und Identifikation für die Menschen, die an der Küste leben oder sie touristisch nutzen. Die

\footnotetext{
${ }^{5}$ Blotevogel (2005, S. 839) verweist auf die definitorische Unschärfe des Raumbegriffs im Raumordnungsgesetz von 1998, in dem ,Raum' sowohl Raum als Erdraum als auch den gesellschaftlichen Raum oder aber unterschiedliche Wirkungszusammenhänge umfassen kann.
} 
schleswig-holsteinische Westküste ist hierfür beispielhaft, da lange Jahrhunderte der Behauptung gegen das Meer eine starke Heimatverbundenheit, Identifikation mit der Kulturlandschaft Marsch und dem Wattenmeer und nicht zuletzt einen ausgeprägten Wunsch nach Selbstbestimmtheit hervorgebracht haben. Die Auswirkungen eines großangelegten Ausbaus der Offshore-Windkraft auf diesen Ort sind vielschichtig und können hier nur ausschnitthaft behandelt werden. Zu vermuten steht allerdings, dass die visuell-ästhetischen Aspekte der OffshoreWindkraft und der sichtbare Wandel der Meereslandschaft im Zuge ihrer vermeintlichen Industrialisierung nicht nur für die Nordseeküstenbewohner eine wesentliche Rolle spielen (vgl. Kempton et al. 2005). Im Folgenden dienen die beiden Küstenkreise Nordfriesland und Dithmarschen als räumliche Verortung des Begriffs (schleswig-holsteinische) „Westküste“ und die Nordsee als Synonym für ,Meer“.

\section{Die Meeresperspektive an der schleswig-holsteinischen Westküste}

\section{Die Westküstenlandschaft}

Landschaft ist ein kompositorischer Begriff und soll auch im Kontext der Westküste als solcher verstanden werden. Während einerseits bestimmte natürliche Formen oder Wirtschaftsweisen einem Gebiet seine charakteristischen landschaftlichen Eigenarten verleihen - im Falle der Westküste zum Beispiel die Eindeichung der Köge - ist andererseits eine Bewusstmachung dieser Eigenarten notwendig, um überhaupt eine Beziehung zur Umwelt entstehen zu lassen (vgl. Burckhardt 1995). Damit die Westküste zur Landschaft werden kann, muss nicht nur die Kulturlandschaft Westküste phänomenologisch geschaffen werden, sondern diese Landschaft dann auch wieder sozial konstruiert und vor allem auch wahrgenommen werden (vgl. Smuda 1986). Dem Westküstenbetrachter eröffnen sich dabei diverse Blickrichtungen. Landschaft ist nicht nur Artefakt, sondern auch Spiegel der Gesellschaft und ihrer Kulturen und nicht zuletzt der sozialen Strukturen der Gesellschaft (vgl. Olwig 2002). Landschaft ist aber auch „Heimat, hat einen Namen oder ist namenlos, besteht aus Orten, die Sammelpunkte des kollektiven Gedächtnisses sind" (Ipsen 2002a, S. 8), und ist somit geprägt von einem immer wieder neu zu schaffenden Sinnbezug zum Raum, der durch reale, symbolische und imaginäre Bedeutungen entsteht, die ihm vom Betrachter bewusst oder unbewusst zugewiesen werden.

\section{Land und Meer in der Westküstenbetrachtung}

Ein wesentliches Element in der sozialen Konstruktion der schleswigholsteinischen Westküstenlandschaft ist die Beziehung des Menschen zum Meer. 
Während einerseits die bewusste Abgrenzung vom Meer und das prekäre Setting zur Identitätsbildung beitragen, sind andererseits die aktive Nutzung des Meeres (beispielsweise durch die Krabbenfischerei) und der maritime Charakter des gesamten Raumes ein Wesensmerkmal des Lebens an der Westküste. Nimmt man die Sichtweisen der Anwohner der beiden Küstenkreise Dithmarschen und Nordfriesland auf das Meer und die Westküste als Ausgangspunkt ${ }^{6}$, so zeigt sich, dass der landseitige Teil der Westküstenlandschaft eher als gestaltete, gelebte Kulturlandschaft wahrgenommen wird, der meerseitige Teil hingegen als natürlich und unberechenbar, der menschlichen Kontrolle trotz aller Nutzung entzogen ${ }^{7}$. Entsprechend ist auch die Bedeutungszuweisung: Die landseitige Kulturlandschaft erfährt Wertschätzung, weil sie eine hart erkämpfte, von Menschenhand gemachte Heimat symbolisiert (vgl. Abb. 3), und die meerseitige Naturlandschaft, weil sie sich - in den Augen der Anwohner - eben jene Unberechenbarkeit und Grenzenlosigkeit erhalten hat. Insbesondere bei dem Aspekt der Grenzenlosigkeit und damit verbunden dem Gefühl der Freiheit spielen die visuellen Qualitäten der Meereslandschaft, ausgedrückt als der freie, weite Horizont eine wichtige Rolle (vgl. Abb. 4).

Ähnliche Ergebnisse liefert eine Analyse der lebensweltlichen Wahrnehmung der Westküste, die Alltagslandschaften, Konfliktlandschaften und bedrohte Landschaften identifiziert (vgl. Meyn 2007). In der Alltagslandschaft, definiert als das Unauffällige und Selbstverständliche des persönlichen Umfelds, wird die Landschaft teils als funktionale, teils als ästhetisch ,schöne' und kontemplative Größe wahrgenommen. Bei der Konfliktlandschaft stehen sich vor allem die beiden Pole ,ökonomische Zwecklandschaft' und Schutzlandschaft Wattenmeer gegenüber. Eine Form der bedrohten Landschaft ist die, verspargelte' Landschaft, in der die Turbinen fast durchweg als Eingriff in die vermeintliche Natürlichkeit der Landschaft gewertet werden (ebd., S. 274) ${ }^{8}$.

\footnotetext{
${ }^{6}$ Diese und die folgenden Aussagen zu Haltungen der Westküstenbewohner beziehen sich auf die eingangs erwähnte Befragung.

7 Typische Antworten auf die Frage „Woran denken Sie, wenn Sie ,Nordsee’ hören“ „Reines Meer, Erholung, Natur, frische Winde, reine Luft, Einfluss auf Seele und Gesundheit, Woblgefübl, gelöst von Hektike und Einengung “„Urgewaltiges, ungebändigtes und noch einigermaßen sauberes Meer, das nicht durch industrielle Nutzung verunreinigt oder verschandelt werden darf. " $\mathrm{Zu}$ den detaillierten Befragungsergebnissen siehe Gee (2009).

${ }^{8}$ Eine niedersächsische Studie hat hingegen gezeigt, dass es zwar deutliche individuelle Meinungsunterschiede gibt, Windparks im Durchschnitt aber nicht als störend empfunden und sogar als charakteristisch für die Küstenregion an der Nordsee gesehen werden (Vogel 2005).
} 


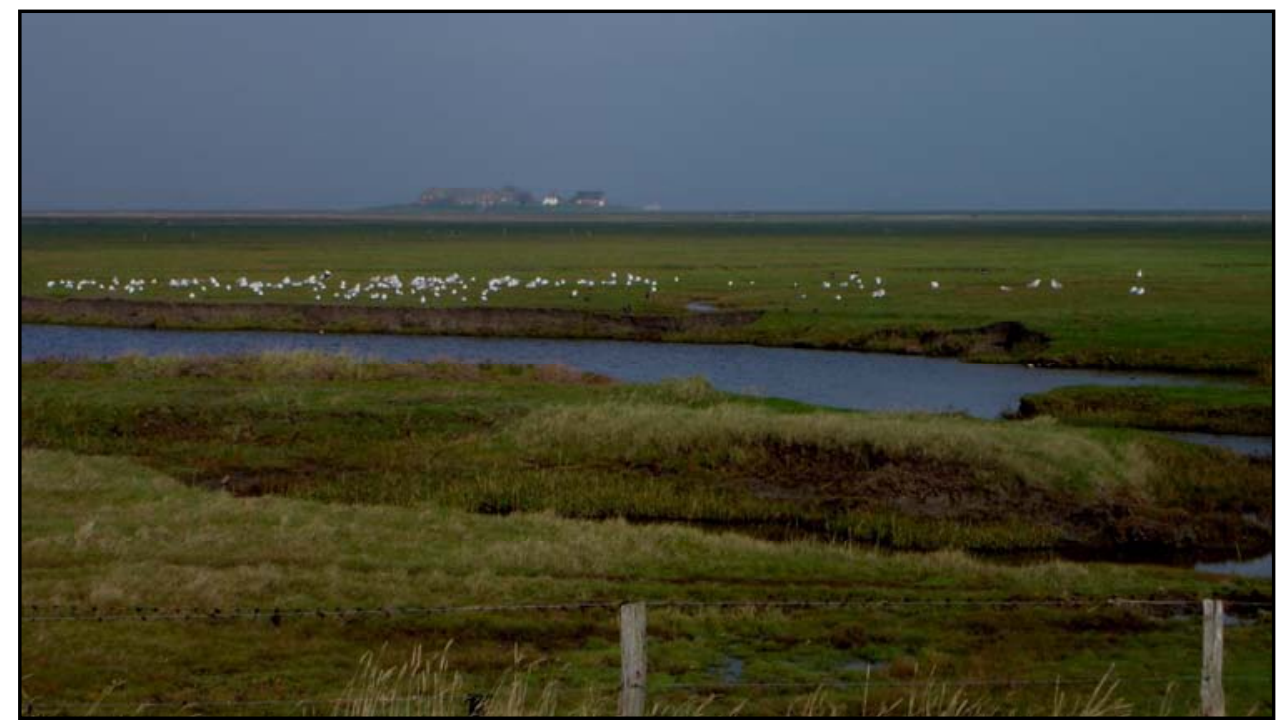

Abb. 3: Kulturlandschaft der schleswig-holsteinischen Westküste - Langeneß (Foto: Gee)

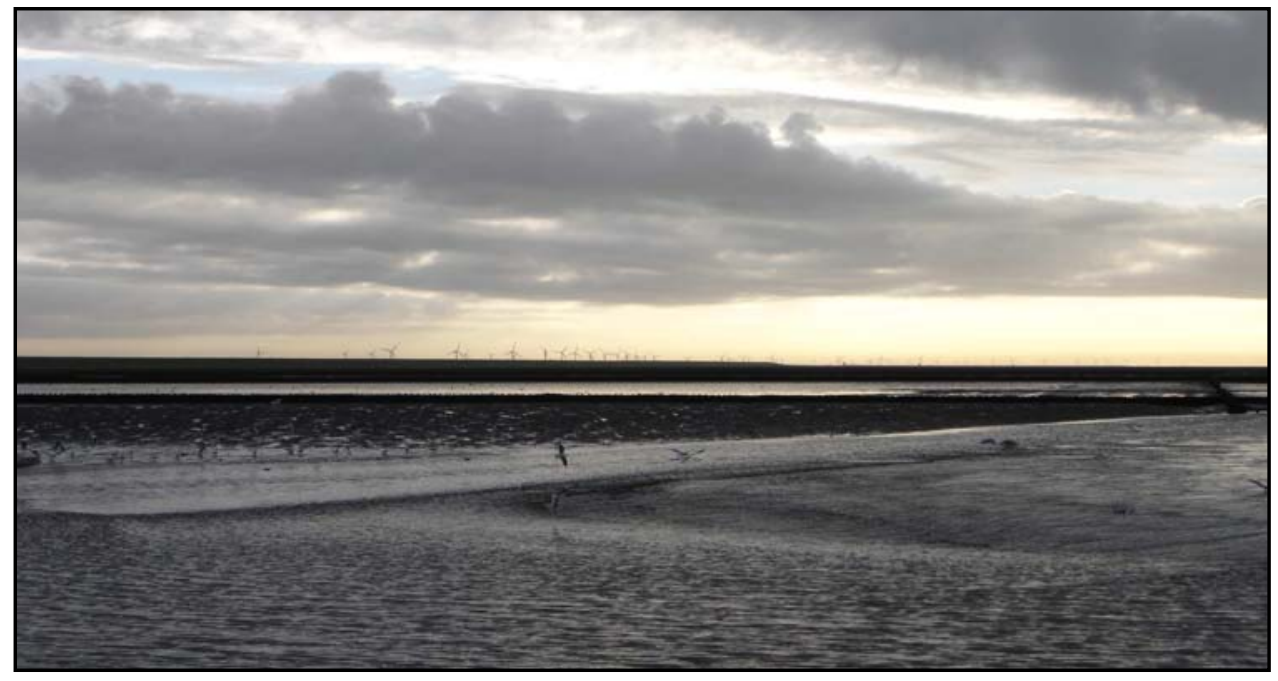

Abb. 4: Der (fast) freie Horizont an der Westküste (Foto: Gee) 
Die Wahrnehmung des Meeres, der Meeres- und der Küstenlandschaft ist eng mit der Forderung nach dem Schutz der jeweils besonderen Qualitäten verbunden. Im Falle der Meeres- und Küstenlandschaft - verstanden hier als die charakteristischen visuellen Qualitäten der Landschaft - stehen eigene Interessen und auch die möglichen Interessen der Tourismuswirtschaft im Vordergrund, die zum großen Teil auf diese Qualitäten bauen. In der Sichtweise des Meeres als Lebensraum steht der Mensch in den Augen der Westküstenbewohner in einer besonderen Pflicht, verwundbare Tiere und Pflanzen im Meer vor Schaden zu bewahren dies ist klar ein ethisches Argument. Wie nun wirken sich speziell OffshoreWindkraftanlagen auf die verschiedenen Sichtweisen des Meeres aus?

\section{Auswirkungen eines Ausbaus der Offshore-Windkraft auf das Meeresbild}

\section{Offshore-Windkraft in der Lebenswelt der Westküstenbewohner}

Nimmt man die Schätzungen der Befragungsteilnehmer als Ausgangspunkt, so deutet viel auf eine eher ambivalente Wahrnehmung der Offshore-Windkraft hin. Zwar sprechen sich ungefähr gleich viele der Befragten für und gegen die Offshore-Windkraft aus, doch sind eindeutige Positionen rarer als unsichere Haltungen, die auf beiden Seiten mit Vorbehalten behaftet sind. Anknüpfend an die oben dargestellte Sichtweise wird die Offshore-Windkraft von einem kleinen Teil der Befragten als gute Alternative zur Offshore-Windkraft betrachtet. Diese Sichtweise ist mit der Hoffnung verbunden, dass sich mit einem Ausbau der OffshoreVariante eine zusätzliche Ausbreitung der Onshore-Windkraft vermeiden oder sogar existierende Windparks abbauen ließen - mit positiven ästhetischen Konsequenzen für die Küstenlandschaft.

Deutlicher ausgeprägt ist jedoch die Sichtweise, dass das Schicksal der Verspargelung des Meeres in jedem Fall vermieden werden muss. Vor allem visuell-ästhetische Argumente werden in diesem Zusammenhang angeführt; die Rede ist häufig von „Verschandelung des Meeres“, dem „Verlust des freien Horizonts“ und einer „räumlichen Zergliederung des Meeres“. Immerhin 56\% der Befragten stufen das weite, offene Meer als sehr wichtig für ihr Leben an der Westküste ein. Bedenken umfassen aber auch die Auswirkungen der Offshore-Windparks auf die Vogelwelt und Meeressäuger oder eine unbestimmte Bedrohung im Sinne von „Zerstörung der Natur“, was wiederum ein Indiz für den hohen Stellenwert des Meeres als Naturraum und den damit verbundenen Schutzgedanken ist.

Dazu kommt die Sorge vor indirekten Bedrohungen wie z.B. der einer Ölpest, die sich aus einem erhöhten Schiffsunfallrisiko ergeben könnte.

Neben diesen eher spezifischen Bedenken kommt jedoch auch ein unbestimmtes Unbehagen zum Ausdruck. Offshore-Windkraftanlagen repräsentieren ein „Gefühl der Eingrenzung“ oder „Verlust all dessen, was mir wichtig ist“, bis hin 
zu „Horror“, „Schreckensvorstellung“ und „Vergewaltigung am Meer“. Gekoppelt an die oftmals poetischen Beschreibungen des Meeres und seiner Wertschätzung als kontemplativer Erholungsraum legt die hier zu Tage tretende Emotionalität nahe, dass dem Meer Werte zugeschrieben werden, die sehr wohl über rein visuell-ästhetische Betrachtungen der Meereslandschaft hinausgehen. Aus der speziellen Wahrnehmung des Meeres und der Wertschätzung bestimmter Meeresqualitäten lassen sich folgende Vermutungen ableiten.

Ein Aspekt ist die symbolhafte Belegung des Meeres mit Konzepten wie Freiheit und Natürlichkeit. Die kontemplative Betrachtung der weiten Fläche, das einfach-in-die-Ferne-Schauen und die gefühlte Unendlichkeit sind mit einem Gefühl der Befreiung und inneren Zufriedenheit verbunden. Obgleich dies eng an den visuellen Eindruck eines unverstellten Horizonts geknüpft ist, zählt jedoch mitunter bereits die Vorstellung vom Meer als offenem, vom Menschen unbeeinflussten Raum. Offshore-Windparks können auch ohne direkte Sichtbarkeit zu einer emotionalen, gefühlten Eingrenzung des Meeres führen (vgl. Kempton et al. 2005).

Ein weiterer Aspekt ist die Tatsache, dass sich die sichtbare Ausprägung des Meeres - in der oberflächlichen Betrachtung jedenfalls - über lange Zeiträume kaum verändert hat. Darin enthalten ist eine implizierte Dauerhaftigkeit, die sich deutlich von den flüchtigen Aspekten menschlichen Lebens abhebt (vgl. Mollat du Jourdin 1993). In dieser Betrachtung wird das Meer zu einer Konstante und zu einem vertrauten Anker, auf den man sich entgegen einer immer schnelllebigeren, veränderlichen Welt auf dem Festland immer wieder berufen kann. Verändert sich die Gestalt des Meeres auf grundlegende wahrnehmbare Weise - wird der vormalige ,Leerraum' beispielsweise durch die Vorstellung fester Industriestrukturen vereinnahmt - bricht diese Illusion der Dauerhaftigkeit in sich zusammen. Die Ablehnung solcher Veränderungen fällt umso heftiger aus, je gravierender sie sich in den Vorstellungen der Anwohner darstellt.

Insgesamt scheinen somit zwei Grundhaltungen relevant, die die eher negativen Reaktionen der Anwohner auf die Offshore-Windkraft erklären helfen: Die eine ist eine kontemplative und zugleich utilitaristische Sicht des Meeres, die die visuell-ästhetischen Eigenschaften des Meeresraums betont und diese an eine Form des persönlichen Gewinns im Sinne von seelischem Wohlbefinden knüpft, z.B. das Gefühl der Freiheit oder des Aufatmens, das sich mit dem Blick auf den unberührten Horizont einstellt. Die andere ist eine eher ethisch geprägte Grundhaltung, die das Meer als verwundbares, wertvolles und schützenswertes Gut begreift. Während sich die Skepsis im Bezug auf Nutzungen in der ersten Grundhaltung hauptsächlich auf sichtbare und vor allem feste Strukturen im Meer bezieht, ist die zweite Grundhaltung allen Nutzungen gegenüber vorsichtig. Nutzungen werden nur dann toleriert, wenn sie das ökologische System Meer - soweit zu beurteilen - nicht negativ beeinträchtigen und die Illusion eines unberührten, ursprünglichen Naturraums nicht zerstören. 


\section{Meereslandschaft und Offshore-Windkraft - ein Tauschgeschäft?}

Die oben angeführten Argumente alleine jedoch führen nicht unbedingt zu einer eindeutigen Ablehnung der Offshore-Windkraft. Natürlich sind es Argumente, die bewusst in die Waagschale geworfen werden. Interessant ist aber, dass trotz der geäußerten Skepsis vieler Anwohner auch dann Unterstützung für die OffshoreWindkraft deutlich wird, wenn die oben angeführten Bedenken explizit erkannt und genannt werden. Was bewirkt diesen Trade-Off? Ein Blick auf die angeführten Argumente macht deutlich, dass dies mit einer prinzipiell positiven Sichtweise der Gewinnung regenerativer Energie zusammenhängt. Offshore-Windkraft steht für eine alternative, saubere, ökologische Energiequelle, die vielfach auch deshalb unterstützt wird, weil sie als Alternative zu Öl, Kohle und Atomkraft betrachtet wird. Die hier geäußerte Unterstützung der Offshore-Windkraft ist somit nicht Offshore-Windkraft-spezifisch, sondern leitet sich aus der Befürwortung erneuerbarer Energien insgesamt ab. Somit wird auf einer stark von Prinzipien bestimmten Ebene argumentiert, auf der der wahrgenommene gesellschaftliche Gewinn über mögliche negative Landschafts- oder Umweltauswirkungen an der Westküste gestellt wird.

\section{Diskussion}

Meeresraum und Meereslandschaft sind wie jedes Raum- oder Landschaftsbild vom Menschen gemacht. Das Beispiel Offshore-Windkraft demonstriert, dass diese Konstruktionen nicht statisch sind, sondern immer wieder neu generiert werden. Landschaftsbewusstsein entsteht aus einem Wechselspiel zwischen der materiellen Umwelt und der bewussten Wahrnehmung von Teilaspekten dieser Umwelt, wobei die Wahrnehmung durch kulturelle, regionale, soziokulturelle oder biographische Faktoren geprägt ist (vgl. Ipsen 2002b). Dass im Blick auf einen Raum sowohl kognitive als auch ästhetische und emotionale Dimensionen zusammenfallen, kann anhand des Meeres anschaulich belegt werden. Die Befragungsergebnisse zeigen, dass die Bewohner aus der Vielfalt der materiellen ,Meeres'-Elemente sehr unterschiedliche Aspekte in ihrer Wahrnehmung herausgreifen. Insgesamt entsteht ein Meeresbewusstsein, in dem die ästhetische Meereslandschaft und die kontemplative Meereserfahrung eine wichtige Rolle spielen. Die persönlichen Wertvorstellungen, die diese Wahrnehmungen motivieren und die gleichzeitig auch Gründe für die Besorgnis im Bezug auf die Offshore-Windkraft darstellen, gründen sich einerseits auf die eigene Lebens- und Erfahrungswelt an der Westküste, andererseits aber auch auf moralische oder ethische Grundhaltungen, die deutlich machen, dass es sich bei den Meeresbildern nicht alleine um utilitaristische Bewertungen handelt. 
Dennoch werden in der Betrachtung der Offshore-Windkraft durch die lokale Bevölkerung auch Brüche in der Sichtweise des Küsten- und Meeresraums sichtbar. Es zeigt sich, dass sich einerseits der Wunsch nach Erhaltung des Meers als ursprünglicher, ,natürlicher' Raum und andererseits der Wunsch nach Nutzung der erneuerbaren Energien gegenüberstehen. Letzteres entspricht weniger dem expliziten Wunsch nach einer Nutzung des Meeres als Energieraum als der Erkenntnis, dass dies aus den vorhandenen Energieoptionen derzeit die beste Lösung darstellt. Ein Meeresbewusstsein, das lange Zeit auf Tradition, bestimmte Formen der touristischen Inszenierung und Kontinuität setzen konnte, wird nun mit einem zweckgebundenen Bild des Meeres konfrontiert, das zwar mit Skepsis betrachtet, jedoch auch aus moralischen gesamtgesellschaftlichen Gründen als unausweichlich eingestuft wird.

Das Unbehagen der Anwohner im Kontext der geplanten Offshore-Windparks deutet auf eine wachsende Divergenz zwischen den neueren Trends und Entwicklungen im Meer und dem eher traditionellen Bild des Meeres hin. Ganz allgemein ist bekannt, dass sich Landschaftsbewusstsein nur langsam ändert und dass Landschaftsveränderungen zu einer Ungleichzeitigkeit zwischen Realität und Wahrnehmung führen können (vgl. Ipsen 2002b). Diese Ungleichzeitigkeit scheint im Falle der Westküstenbewohner erreicht. In dem Sinne, dass sich die Gestalt des Meeres unwiderbringlich verändern wird, stellt die Offshore-Windkraft einen ,tipping point' dar, der sich nicht nur im materiellen Meeresraum manifestiert, sondern auch unausweichlich auf die Meeresbilder im Kopf wirkt. Dies liegt daran, dass diese Formen der Veränderung nicht mehr bewusst ausgeblendet werden können. Veränderungen im Meer wie Offshore-Windparks sind präsent, großflächig, wahrnehmbar und stehen zunächst noch ohne bewusste Inszenierung oder romantischer Verklärung. Anteil an dem latenten Unbehagen hat sicherlich auch die Tatsache, dass Offshore-Windkraft zudem einen tiefgreifenden Wandel sichtbar macht, der sich nicht nur auf die Westküste, sondern die globale Gesellschaft bezieht. Offshore-Windkraft drängt mit neuen, ungewohnten Fragen: Woher kommt unsere Energie, kann der Klimawandel gestoppt werden? Welchen Meeresraum wollen wir, welcher Meeresraum ist möglich, und wer steuert diese Entwicklungen?

Es sind jene Fragen, die im Kontext der aktuellen Entwicklung im Meer nicht umfassend beantwortet werden. Offshore-Wind ist das Ergebnis expliziter Gunstfaktoren auf der ökonomischen, gesellschaftlichen und politischen Ebene; Versuche einer integrierten Steuerung wie z.B. durch eine Meeresraumordnung sind eher nachgelagert. Was fehlt ist eine bewusste Entscheidung für eine Entwicklungsoption im Meer statt einer Entwicklung, die tendenziell von externen Faktoren befördert und von den regionalen und nationalen Entscheidungsträgern aufgegriffen wird. Diese Entscheidung muss mit Blick auf das Meer nicht nur als Nutzungsraum, sondern auch als Landschaft und Ort getroffen werden, da hier entscheidende Weichen für die Zukunft gestellt werden. Offshore-Windkraft bietet eine akute Diskussionsgrundlage, weil die Schnittmengen zwischen den Wert- 
vorstellungen, Interessen und Sorgen ganz unterschiedlicher Akteure immerhin auf einige Konvergenzen hoffen lassen. 


\section{Literatur und Quellen}

Blotevogel, H. H. (2005): Raum. In: Handwörterbuch der Raumordnung. 4. Aufl. Hannover: ARL. S. 831-841.

Bundesministerium für Umwelt (BMU) (2007), in Zusammenarbeit mit der Stiftung der deutschen Wirtschaft zur Nutzung und Erforschung der Windenergie auf See: Entwicklung der Offshore-Windenergienutzung in Deutschland. Broschüre, 29 S., Januar 2007.

Bundesministerium für Umwelt (BMU) (2002): Weiterer Ausbau der Windenergienutzung im Hinblick auf den Klimaschutz, Teil 2. Erarbeitet im Rahmen des F\&E Vorhabens 99946101 vom Deutschen Windenergie-Institut GmbH, Wilhelmshaven. November 2002. Bundesministerium für Umwelt, Naturschutz und Reaktorsicherheit, Bonn/Berlin.

Bundesministerium für Wirtschaft und Arbeit (BMWi) (2008): Schlaglichter der Wirtschaftspolitik, Monatsbericht 04/2008. www.bmwi.de, letzter Zugriff am 6. Mai 2008.

Bruns, A. u. Gee, K. (2009): From State-centred Decision-Making to Participatory Governance - Planning for Offshore wind farms and the Implementation Process of the Water Framework Directive in Northern Germany. GAIA 18/2, S. 150-157.

Bundesamt für Seeschifffahrt und Hydrographie (BSH) (2009): Genehmigte Windparks in der Nordsee. http://www.bsh.de/de/Meeresnutzung/Wirtschaft/Windparks/index.jsp, letzter Zugriff am 5. Mai 2009.

Burckhardt, L. (1995): Design ist unsichtbar. Ostfildern.

Byzio, A., Mautz, R. u. Rosenbaum, W. (2005): Energiewende in schwerer See? Konflikte um die Offshore-Windkraftnutzung. 180 S., oekom verlag, München.

Douvere, F. u. Ehler, C. (2009): New perspectives on sea use management: Initial findings from European experience with marine spatial planning. Journal of Environmental Management 90, S. 77-88.

Fischer, N., Müller-Wusterwitz, S. u. Schmidt-Lauber, B. (2007): Inszenierungen der Küste. Schriftenreihe der Isa Lohmann-Siems Stiftung, Band 1. Reimer Verlag. Berlin.

Gee. K. (2009): Offshore wind power development as affected by seascape values on the German North Sea coast. In: Land Use Policy (13.06.2009) 
Gee, K., Kannen, A., Licht-Eggert, K., Glaeser, B. u. Sterr, H. (2006): Integriertes Küstenzonenmanagement (IKZM): Raumordnungsstrategien im Küstenbereich und auf dem Meer. Forschungsprojekt des BMVBS/BBR. Abschlussbericht, Berlin, Oktober 2006.

Ipsen, D. (2002a): Landschaftsbewußtsein in der Lausitz. Ergebnisse der Umfrage. Band III. Arbeitsgruppe Empirische Planungsforschung, Universität Kassel.

Ipsen, D. (2002b): Raum als Landschaft. In: Kaufmann, S. (Hrsg): Ordnungen der Landschaft: Natur und Raum technisch und symbolisch entwerfen. Identitäten und Alteritäten; Band 12, Ergon Verlag, Würzburg, S. 33-60.

Kempton, W., Firestone, J., Lilley, J., Rouleau, T. u. Whitaker, P. (2005): The offshore wind power debate: views from Cape Cod. Coastal Management 33, S. 119-149.

Kommission der Europäischen Gemeinschaften (2007): Mitteilung der Kommission an das europäische Parlament, den Rat, den Europäischen Wirtschaftsund Sozialausschuss und den Ausschuss der Regionen: Eine integrierte Meerespolitik für die Europäische Union. KOM (2007) 575 endgültig, Brüssel, 10.10.2007.

Licht-Eggert, K., Gee, K., Kannen, A., Grimm, B. u. Fuchs, S. (2008): The human dimension in ICZM: Adressing peoples perceptions and values in integrative assessments. In: ICZM - The Global Challenge. Edited by Krishnamurthy, R., Kannen, A., Alagappan, R. Glavocic, B., Green, D., Han, Z., Tinti, S. u. Tundi, A.: Research Publishing, India, S. 241-262.

Ministerium für Arbeit, Bau und Landesentwicklung Mecklenburg-Vorpommern (2005): Landesraumentwicklungsprogramm Mecklenburg-Vorpommern. Schwerin, August 2005.

Ministerium für Wissenschaft, Wirtschaft und Verkehr des Landes SchleswigHolstein (2007): Initiative Zukunft Meer. Broschüre, erschienen am 26.11.2007.

Meyn, J. (2007): Biographische Küstenlandschaften. In: Fischer, N., MüllerWusterwitz, S. u. Schmidt-Lauber, B. (2007): Inszenierungen der Küste. Schriftenreihe der Isa Lohmann-Siems Stiftung, Band 1. Reimer Verlag. Berlin. S. 259-280.

Mollat du Jourdin, M. (2003): Europa und das Meer. C. H. Beck Verlag, München.

Olwig, K. (2002): Landscape, Nature, and the Body Politic: From Britain's Renaissance to America's New World. University of Wisconsin Press.

Smuda, M. (1986): Natur als ästhetischer Gegenstand und als Gegenstand der Ästhetik. Zur Konstitution von Landschaft. In: Smuda, M. (Hrsg): Landschaft. Suhrkamp Verlag, Frankfurt a.M., S. 44-69. 
Nutzungswandel historischer Gartenanlagen am Beispiel der aktuellen Debatte über den Park Kassel-Wilhelmshöhe 


\begin{abstract}
This article deals with tourist concepts with regard to the historical garden Wilhelmshöhe close to Kassel-city. In recent times it was vividly discussed how the garden should be presented to the public and visitors with a view to increased economic efficiency. More-over, it is intended to put the park on the Unesco World Heritage-list. In this context, several initiatives have been started, carried especially by Kassel's public e.g. civic associations and the chamber of commerce. A look on the historical use of the garden shows that e.g. in the 18th century a variety of interpretations and uses of the park was possible or intended. That leads to considerations on how future visitors can be given an impression of the garden's historical development and make them curious about its unique character. The aim of future tourist concepts should be a sustainable understanding and identification with the garden as a cultural heritage worth to be protected.
\end{abstract}

\title{
Einleitung
}

Derzeit befindet sich der Bergpark Kassel-Wilhelmshöhe in einer Umbruchphase hinsichtlich der künftigen Nutzung. Einerseits ist man bestrebt, den Park mit seinen einzigartigen Wasserspielen unter dem Motto „Technik - Kunst - Natur“ als Unesco Weltkulturerbe anerkennen zu lassen (vgl. Abb. 1). Andererseits wird wirtschaftlich die Notwendigkeit proklamiert, den Park touristisch offensiver zu erschließen, um sowohl der Stadt als auch der Region mehr Einnahmen zu verschaffen und um ihr Image so aufzuwerten, dass an das Prestige etwa von Dresden, Berlin und München Anschluss gefunden werden kann.

Diese ganz unterschiedlichen Zielvorstellungen prallten in den vergangenen Jahren in Kassel aufeinander. Zwar sollen alle Konzepte zum Erhalt, zur Vermittlung und zur „Öffnung“ der historischen Gartenanlagen für größere Besuchermengen an die Richtlinien der Gartendenkmalpflege bzw. der Unesco gebunden sein. Gleichzeitig werden jedoch die Erfordernisse des Stadtmarketings betont, wobei sich der grundsätzliche Konsens abzeichnet, dass gerade historische Gartenanlagen das Image einer Stadt positiv zu beeinflussen vermögen. Dieses Spannungsfeld zwischen ökonomischen und denkmalpflegerischen Erfordernissen ist keineswegs singulär und wird im Sammelband „Marketing für Gärten und Schlösser" (2004) von Brandt, v. Bothmer und Rohde vielschichtig thematisiert. Die aus diesem Konflikt resultierenden, ganz unterschiedlichen Ansprüche an die Umgangsweise mit historischen Gärten sollen im Folgenden am Beispiel der Debatte über Kassel-Wilhelmshöhe gezeigt werden.

Zunächst ist zu schauen, welche Zukunftsvisionen das Land Hessen hinsichtlich der Kasseler Gartenanlage(n) vertritt. Des Weiteren wird auf das Bestreben 
eingegangen, den Park als Unesco-Weltkulturerbe einstufen zu lassen - ein Vorhaben, das von einer aktiven Bürgerbeteiligung und einer regionalen Netzwerkbildung begleitet wird. Anschließend soll gezeigt werden, wie unterschiedlich die Gartenanlage etwa im 18. Jahrhundert genutzt und gedeutet wurde, um dann schließlich für die Entwicklung eines nachhaltigen Tourismuskonzepts für den Garten zu plädieren.

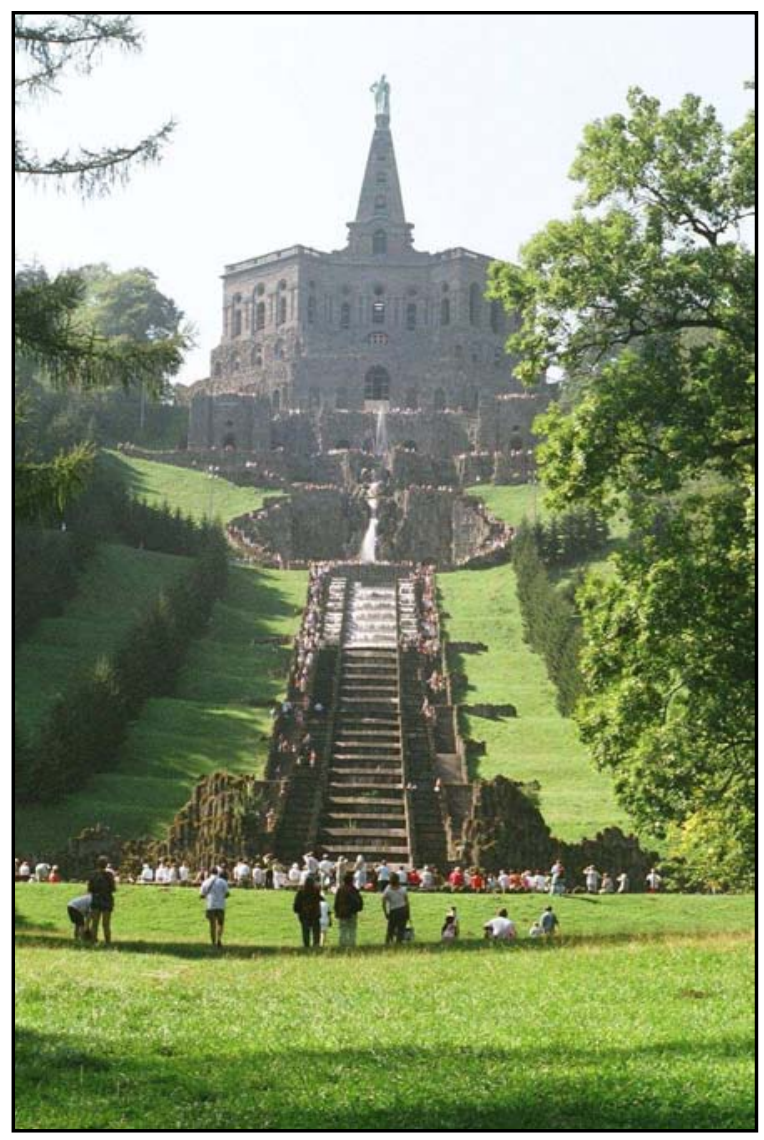

Abb. 1: Das Foto zeigt die barocke Kaskade, die den Anfang der rund 60 Minuten währenden historischen Wasserspiele im Park bildet (Foto: Stobbe)

\section{Das Zukunftskonzept und die Gründung der Museums- landschaft Hessen Kassel (mhk)}

Auslöser für die bis heute andauernde Diskussion über die Zukunft des Parks war die Eingabe der Hessischen Landesregierung, alle Kulturgüter des Landes auf den 
Prüfstein der ökonomischen Verwertbarkeit zu stellen. Dabei wurde das große Potenzial Kassels erkannt - einer Stadt, die im 18. Jahrhundert bedeutende Residenzstadt war. Zwar verfügt Kassel über eine Vielzahl historisch hochwertiger Kunstsammlungen sowie mit dem Nachlass der Brüder Grimm schon jetzt über ein Unesco-Weltdokumentenerbe, doch haben die weit reichenden Zerstörungen während des Zweiten Weltkriegs und die zum Teil fragwürdigen Neubauten der 1950er und 1960er Jahre das Image der Stadt gemindert. Einzig während der alle fünf Jahre stattfindenden „Documenta“ kann Kassel auf nationaler und internationaler Ebene von sich reden machen.

Das soll sich nun nach Vorstellungen des Hessischen Ministeriums für Wissenschaft und Kunst in Wiesbaden ändern. So wurde im Jahr 2004 ein Gemeinschaftsauftrag für das Erstellen eines Konzepts zur Neuordnung der Kasseler Museumslandschaft vergeben, das im Jahr 2005 von der Arbeitsgemeinschaft Albert Speer \& Partner und bogner.cc (cultural consulting) vorgelegt wurde. Das Kulturmarketingbüro bogner.cc in Wien agiert ebenso wie das Architektur- und Stadtplanungsbüro „Albert Speer \& Partner“ auf internationaler Ebene. Gemeinsam haben sie einen „museologischen Masterplan“ oder „Museumsleitplan“ entwickelt, der eine Umorganisation der Kasseler Kunstsammlungen unter Einbeziehung der Kasseler Garten- und Schlossanlagen vorsieht.

Der rund 320 Seiten umfassende Bericht, der auf der Homepage des Hessischen Ministeriums für Wissenschaft und Kunst einsehbar ist (http1), enthält zahlreiche Vorschläge, die sich bis heute als (kultur-)politisch brisant erweisen. Vor allem die Tatsache, dass zur Entwicklung dieses Konzepts keine Expertise seitens der Denkmalpflege oder der Gartendenkmalpflege im Speziellen eingeholt worden war, hat für viel Kritik gesorgt. Auch die Planungen der Deutschen Marketing Gesellschaft (DMG) hinsichtlich des künftigen Besucheraufkommens haben Anlass zur Diskussion gegeben.

Nach Auffassung der DMG sollen gemäß ihrem knapp 90-seitigen Marketingkonzept zunächst einmal Kulturinteressierte, Kulturreisende und Eventtouristen angesprochen werden. Als besonderes Verhaltensmuster wird das Bedürfnis nach dem „Besonderen“ oder „Nichtalltäglichen“ angenommen. Die Besucher erwarten laut DMG eine ,zeitgemäße Heranführung“ an das Kulturgut, kulinarische „Belohnungen“ und insgesamt ein auf Erlebnis und Ambiente ausgerichtetes Tourismuskonzept. Kurz: „Eine verwöhnte Erlebnisgeneration will in inszenierten Highlights baden“ (DMG 2004, S. 23f.). Mit verschiedenen Werbestrategien sollen neue Zielgruppen als potenzielle Besucher hinzugewonnen werden. Insbesondere von den rund 5,6 Mio. Fernreisenden, die jährlich mit dem ICE den Bahnhof Kassel-Wilhelmshöhe passieren, sollen $2 \%$, das sind 112.000, mit Slogans wie „Zum kulturellen Auftanken auf die Wilhelmshöhe“ oder „Have a break in Kassel“ zum Pausieren in den Park gelockt werden (vgl. ebd., S. 29f.).

Es wird seitens der DMG davon ausgegangen, dass momentan ca. 230.000 Besucher jährlich nach Kassel kommen, von denen 175.000 die Wilhelmshöhe besuchen. Diese Zahl soll noch einmal, nach verschiedenen Besuchergruppen gestaf- 
felt, um weitere 570.000 auf ein Potenzial von 800.000 Gästen erhöht werden, wobei dazu auch kulturferne Besuchergruppen anvisiert werden. Rund 55 Mio. Euro Mehreinnahmen pro Jahr sind prognostiziert, die durch Hotelübernachtungen, Ausgaben in den Museen, Verpflegung, Dienstleistungen und sonstige Einkäufe in der Stadt eingenommen werden sollen (vgl. DMG 2004, S. $31 \mathrm{ff}$.).

Diese Zahlen mögen auf die Bewohner der Stadt Kassel verlockend und abschreckend zugleich wirken. Würden tatsächlich 800.000 Besucher jährlich nach Kassel kommen, wäre anteilsmäßig mit rund 600.000 Besuchern auf der Wilhelmshöhe zu rechnen. Das machte ungefähr ein Drittel der Besucher aus, die jährlich in Sanssouci (Potsdam) gezählt werden (2.000.000), aber auch an die dreimal so viel wie in Stourhead (252.000) - um zwei prominente Vergleichsgrößen zu wählen (vgl. Tab. 1). Angesichts der Tatsache, dass es zwar eine geteerte Straße durch den Park gibt, die weitestgehend den Stationen der Wasserspiele folgt, ansonsten aber bislang noch kein durchdachtes Besucherleitkonzept für den Park selbst entworfen worden ist, kann man die Sorge nachvollziehen, dass eine mögliche Zerstörung der kleinteiligen Wegestruktur zu befürchten ist.

Tab. 1: Besucherzahlen in ausgewählten historischen Gärten Europas. Quelle: Antz 2007, S. 63.

\begin{tabular}{l|l}
\hline Parkanlage & Besucherzahlen pro Jahr \\
\hline Sanssouci (Potsdam) & 2.000 .000 (April bis Oktober) \\
Bad Muskau (Deutschland-Polen) & 250.000 \\
Bremer Bürgerpark (Bremen) & 1.000 .000 \\
\hline Herrenhausen/Großer Garten (Hannover) & 220.000 (im Winter kein Eintritt) \\
Versailles (Frankreich) & 7.000 .000 \\
Hampton Court Gardens (London) & 1.200 .000 \\
Stourhead (England) & 252.000 \\
Lost Gardens of Heligan (England) & 370.000 \\
Schlosspark Schönbrunn (Wien) & 6.700 .000 \\
Belvederegärten (Wien) & 2.300 .000 \\
Augarten (Wien) & 1.200 .000 \\
Burg- und Volksgarten (Wien) & 6.200 .000 \\
\hline Landschaftspark Laxenburg (Österreich) & 1.000 .000 \\
Schloß Hellbrunn (Österreich) & 350.000 \\
\hline Schlossgarten Cesky Krumlov (Tschechien) & 600.000 \\
Boboli Garten (Florenz) & 800.000 (Nichtflorentiner) \\
\hline
\end{tabular}


Zudem stellt sich die Frage nach der Verkehrsanbindung und einer altersgerechten Fortbewegung im Park selbst. Ob eine Straßenbahn, ein Bus oder aber Elektroautos durch den Park fahren sollen oder dürfen, ist bis heute ungeklärt. Inwiefern ehemalige Parkstraßen für den Autoverkehr freigegeben und Hofgebäude des ehemaligen Wirtschaftsbereichs umfunktioniert werden können, ist ebenso umstritten wie die Frage nach „Snackpoints“ im Park oder Veranstaltungen wie Autorallyes. Auch die Frage, wo, wie viele und in welcher Ausführung Besucherzentren im Park gebaut werden sollen, ist lange Zeit Gegenstand der Diskussion gewesen. Mittlerweile ist ein Besucherzentrum bei der Endhaltestelle der Straßenbahnlinie 1 fertig gestellt worden. Über den Standort des zweiten Besucherzentrums ist derzeit noch nicht entschieden worden.

Andere Punkte, die das Zukunftskonzept vorsieht, werden hingegen einhellig begrüßt. Dazu zählt allem voran die hohe Investitionssumme, die in den nächsten Jahren unter anderem für die Sanierung zahlreicher Parkgebäude ausgegeben wird. Das Land Hessen zahlt 200 Mio. Euro und laut Pressemitteilung vom 27.02.2009 steuert die Stadt Kassel 20 Mio. Euro zum Erhalt und zur Sanierung bei - eine Summe, die seit der Nachkriegszeit nicht mehr in den Kulturetat geflossen ist, was den hohen Stellenwert dieses Prestigeobjekts zeigt.

Neben dem Beginn der Sanierungsarbeiten wurde mittlerweile eine neue Dachorganisation, die „Museumslandschaft Hessen Kassel (mhk)“ (http2), im Jahr 2006 gegründet. In dieser sind nicht nur die Sammlungen der vormaligen Institution „Staatliche Museen Kassel“, sondern erstmals auch die historischen Gartenanlagen Wilhelmshöhe, Karlsaue und Wilhelmsthal bei Kassel-Calden vereint. Seit 1946 unterstanden diese Anlagen samt den in ihnen befindlichen Objekten der Direktion der „Staatlichen Verwaltung der Schlösser und Gärten Hessen“ in Bad Homburg. Bereits 1918 war der Besitz in eine Vorgänger-Institution der ehemaligen preußischen Provinz Hessen-Nassau übergegangen.

Mit dieser weit reichenden Umstrukturierung wird der Hoffnung Rechnung getragen, dass sich historische Gartenanlagen besser vermarkten lassen, wenn sie zentral vor Ort verwaltet und in ein städtisches Gesamtkulturprogramm mit den unterschiedlichsten Sammlungen und Schwerpunkten integriert werden. Vor allem aber auch deshalb, weil so die Präsentation von verschiedenen hochrangigen Gemälde- und Antikensammlungen etwa in Schloss Wilhelmshöhe besser mit einem Besuch im Park verschränkt werden kann, ist dieser Schritt zu begrüßen. Ob es jedoch der Anlage gerecht wird, sie nach Auffassung der Planer in einen so genannten Museumspark zu verwandeln, bleibt ebenso zweifelhaft, wie das Vorhaben, auf Schloss Weißenstein einen „fürstlichen Kosmos“ zu konstruieren - denn beides wäre letztlich ahistorisch.

Wie bereits angedeutet wurde, ist gerade die Umfunktionalisierung dieses Parks in einen Touristenmagneten und leicht zugänglichen Belustigungsort "für alle“ nicht unumstritten, da dies der Bestrebung, den Park als Unesco-Weltkulturerbe einstufen zu lassen, durchaus entgegensteht, wenn an den bisherigen Planungen uneingeschränkt festgehalten wird. Denn um den Park als Unesco-Weltkulturerbe 
einstufen zu lassen, muss laut „Übereinkommen zum Schutz des Kultur- und Naturerbes der Welt" der Unesco (1972) die Unversehrtheit und Authentizität des Denkmals nachgewiesen und dessen Erhalt garantiert werden.

\section{Die Unesco Welterbe-Initiative: Von Bürgerbeteiligung bis Netzwerkbildung}

Der Verein Bürger für das Welterbe e.V. tritt seit seiner Gründung im Jahr 2001 maßgeblich für den Welterbe-Antrag der Kasseler Parkanlagen und die Einhaltung der Unesco-Richtlinien bei der Entwicklung neuer Gartennutzungskonzepte ein. Durch verschiedene Veranstaltungen und eine eigene Homepage (http3) bezieht er kritisch Stellung zu den einzelnen Planungspunkten. Auf Drängen des Vereins wurde das Landesdenkmalpflegeamt Hessen in Wiesbaden von offizieller Seite damit beauftragt, die Antragstellung bei der Unesco durchzuführen und dafür Sorge zu tragen, dass bei den weiteren Planungen nicht gegen die Kriterien der Unesco zur Vergabe des Titels „,Weltkulturerbe“ verstoßen wird.

Die Akzeptanz der Umstrukturierungspläne bei der Kasseler Bevölkerung ist letztlich Dreh- und Angelpunkt aller Konzepte. Schon bei der Beurteilung von Welterbeanträgen legt die Unesco mittlerweile Wert darauf, dass dieses Bestreben auch von der ansässigen Bevölkerung getragen wird, das heißt, dass sie sich mit diesem Kulturgut identifizieren kann. Unterstützt wird diese Bürgerbeteiligung nicht zuletzt von zahlreichen Kasseler Vereinen wie dem Museumsverein, dem Rosenverein, dem Verein der klassischen Antike etc., die an einem runden Tisch Gespräche führen und gemeinsame Veranstaltungen planen und durchführen. Entsprechend dem oben erwähnten Zukunftskonzept fanden, wenn auch teilweise erst auf Bestreben des Vereins Bürger für das Welterbe, Workshops zur Einbindung der Bevölkerung in die Planungsvorhaben statt. Ende Februar 2009 wurde zudem eine von Land und Kommune getragene Kampagne „Kultur baut auf. Wir bauen um.“ im Internet gestartet (http4), die über den aktuellen Stand der Bauarbeiten informiert. Dennoch gibt es auch weiterhin grundlegende Interessenskonflikte.

Denn auch wenn historische Gartenanlagen derzeit über einen hohen ästhetischen und monetären ,freizeit- und tourismusbezogenen Gebrauchswert“ (Hlavac 2005, S. 6) verfügen und als Ressource angesehen werden, ist ihre Nutzung je nach Betrachterperspektive mit ganz unterschiedlichen Wertvorstellungen verbunden: „Touristen und Einheimische wollen die Garten- und Parkanlagen zu jeder Zeit besichtigen können und erwarten [...] die Anlagen ohne Baustellen oder laufende Arbeiten am pflanzlichen Bestand; Denkmalpfleger versuchen die verschiedenen zeitlichen Schichten einer Anlage im Bestand darzustellen, der Erhalter steht im Spannungsfeld zwischen ökonomisch gerechtfertigter Erhaltung, den Ansprüchen der Denkmalpflege und dem zunehmenden Nutzungsdruck und die 
Tourismuswirtschaft hat ein Interesse die Anlagen so vielen Kunden wie möglich als touristische Attraktion anbieten zu können“" (Ebd., S. 7).

Als fruchtbarer und konstruktiver Lösungsansatz wird im Bereich der touristischen Nutzung historischer Gartenanlagen die Bildung von Netzwerken angesehen. Es gilt insgesamt das Credo, dass sich Tourismus- und Marketingkonzepte nur in der Kooperation mit möglichst vielen regional eingebundenen Verbänden, Institutionen und Vereinen umsetzen lassen. Der Sammelband „GartenNetze Deutschland" von Brandt, v. Bothmer und Mangels (2007) führt eine Reihe von Projekten auf, die alle in diesem Kontext stehen.

Während in Deutschland das Fehlen einer bundesweit operierenden Gartenorganisation bemängelt wird, sei es hingegen in England dem „National Trust“" und der „Royal Horticultural Society“ (RHS) gelungen, britische Gärten „qualitätvoll, wirtschaft- und kundenfreundlich" (Klaffke 2007, S. 45) zugänglich und bekannt zu machen. An diesem Vorbild könne man sich nach Auffassung Klaffkes in Deutschland orientieren und in abgewandelter Form ein „Gartennetz Deutschland“" als eine Art Dachverband aufbauen, der jedoch die regionalen Besonderheiten und Eigenständigkeiten nicht einschränken oder gar einebnen soll (ebd., S. 48). Über jährliche Treffen und die Einrichtung einer Gartenplattform im Internet soll diese Idee verankert werden und sich immer weiter ausbreiten. Dabei könne auch auf die Erfahrungen und Kontakte der Organisationen Deutsche Gesellschaft für Gartenkunst und Landschaftskultur (DGGL) und Deutsche Gartenbau Gesellschaft (DGG) zurückgegriffen werden.

Wie Ringkamp (2007, S. 52f.) darlegt, liegt die Besonderheit im deutschen Kontext darin, dass sich Ende der 1990er Jahre an die 20 Initiativen gegründet haben, die von Ministerien, Verbänden, Vereinen, Stiftungen, städtischen und landeseigenen Marketinggesellschaften oder Tourismusverbänden getragen werden. In Sachsen-Anhalt ist das beispielsweise das Projekt „Gartenträume - Historische Parks in Sachsen-Anhalt“ (seit 1999), das aus dem 2003 der Verein „Gartenträume“ hervorgegangen ist. Des Weiteren sind die Initiativen „gartenrouten zwischen den meeren“ (seit 2007) oder „Gartenregion Hannover 2009“ (seit 2006) zu nennen. Die Ziele klingen stets ähnlich: Die Gärten sollen erhalten und nach außen (Tourismus) und innen (regionale Identität) bekannt gemacht werden; man will sich durch ein besonderes (Kulturlandschafts-) Image von anderen Regionen abgrenzen und allgemein neue Gartenliebhaber gewinnen, mit denen man sich austauschen, vernetzen, informieren und beraten kann. In der Regel werden spezielle Themenschwerpunkte für die unterschiedlichen Routen gebildet oder Kombinationen mit anderen Themen hergestellt, die noch andere Interessen der potenziellen Besucher ansprechen (Bewegung, andere Künste, kulturelle und wissenschaftliche Veranstaltungen, kulinarische Genüsse).

Darüber hinaus gibt es seit 2003 das Europäische Gartennetzwerk European Garden Heritage Network (EGHN), das von der EU als Interreg-IIIB-Projekt gefördert wird (vgl. Spanjer 2007, S. 31). Hier lautet das Ziel: „das gartenkulturelle Erbe Europas sichern, weiterentwickeln und bekannter machen". Vernetzt sind 
darin derzeit über 220 Gärten, Parks und Museen in ganz Europa, wobei es bestimmte Schwerpunktregionen wie etwa Ostwestfalen-Lippe, das Ruhrgebiet, Münsterland und das Rheinland in Deutschland, Surrey, Cheshire und Somerset in Großbritannien, Pays de la Loire in Frankreich sowie Westflandern in Belgien und Limburg in den Niederlanden gibt. Wie der Internetpräsentation der EGHN zu entnehmen ist, wird seit November 2008 das Konzept einer EGHN-Route NordHessen entwickelt (http5).

Einer der zentralen Initiatoren für diese NordHessen-Route ist die Industrieund Handelskammer (IHK) Kassel. Sie hat es sich seit 2007 zur Aufgabe gemacht, nicht nur den Welterbeantrag der Wilhelmshöhe zu unterstützen, sondern auch als Promotor des Weltdokumentenerbes der Menschheit (Memory of the World) „Grimms Märchensammlungen“ aufzutreten. Die Nordhessen-Route steht im Kontext der anderen regionalen Routen zum Themenkomplex „Gartenkunst, Kulturlandschaft, regionale Identität“ und soll bis Mai 2009 ausgearbeitet sein. Ziel dieser regionalen Routen ist es, bestimmte Gärten als Ausgangspunkt (als „Ankergarten“) zu nutzen, „um Gartenkunst, Natur, Geschichte und regionale Identität zu erleben, Neues und Verborgenes zu entdecken, Altbekanntes mit anderen Augen zu sehen und das Besondere jeder Kulturlandschaft zu genießen“ (http6). Bei der NordHessen Route soll die Wilhelmshöhe als ein solcher Ankergarten fungieren.

Die Industrie- und Handelskammer (IHK) kann neben der Museumslandschaft Hessen Kassel (mhk), der Universität Kassel und der NordHessen Touristik (NHT) zudem als treibende Kraft bei der Konzeption des Gartennetzwerks Gartenregion Nord-Hessen gelten. Dieser Prozess soll im Sommer 2009 abgeschlossen sein. Ziel ist es, „eine optimale Ausschöpfung der ökonomischen Potenziale der Gartenkunstwerke zu erreichen“, wie Koseck und Schlosser (2008, S. 24) anlässlich des Auftaktsymposiums im Mai 2008 hervorheben. Der Welterbestatus kann dafür nur förderlich sein, wie in mehreren Beiträgen in den darauf folgenden Ausgaben der Zeitschrift ,Wirtschaft Nordhessen“ betont wird.

Dieses Engagement trägt dem Trend Rechnung, dass Gartentourismus etwa in England die drittstärksten Wachstumsraten aufweist (Antz 2007, S. 71). Diese Art „Gartenboom“ spiegelt sich auch in anderen Bereichen (Zeitschriften, Gartensendungen, Gartenbedarf) und Ländern (Frankreich) wider. Nicht ohne Grund hat die Deutsche Zentrale für Tourismus (DZT) im Jahr 2008 das „Themenjahr der Schlösser, Parks und Gärten“ ausgerufen. Zwar lassen sich flächendeckend nur schwer Zahlen ermitteln, jedoch zeigt sich die steigende Nachfrage zumindest exemplarisch in einzelnen Regionen anhand primärer Effekte (stärkerer Umsatz im Hotel- und Gaststättengewerbe) und weicher Standort- und Imagefaktoren (Bekanntheit). Neben einer wirtschaftlichen Wertschöpfung ist damit auch die Schaffung neuer Arbeitsplätze nicht nur im Bereich der Gartenbaufirmen, der Landschaftsplaner und im Gastgewerbe, sondern auch im Bereich der Besucherbetreuung verbunden (ebd., S. 73). 
Gerade diese Dominanz der ökonomischen Verwertbarkeit auf unterschiedlichen Ebenen ruft jedoch auch immer wieder Misstrauen bis hin zu Missmut hervor, zu deutlich scheint dieser Nutzungsaspekt dem eigentlichen Ziel von Gärten - nämlich zur Erbauung, zum Vergnügen oder zum zweckfreien Kunstgenuss angelegt worden zu sein - zu widersprechen. Doch wie war das eigentlich ursprünglich; nach welchen Kriterien wurde etwa Park Kassel-Wilhelmshöhe angelegt; welchen Zwecken sollte er dienen?

\section{Historische Nutzung von Kassel-Wilhelmshöhe im 18. Jahrhundert}

Zunächst einmal muss festgehalten werden, dass Park Kassel-Wilhelmshöhe ein hochadeliger Lustgarten war und in dieser Ausprägung deutlich von Gärten des Kleinadels oder des entstehenden Bildungsbürgertums zu unterscheiden ist. Entscheidend ist dabei die Funktion als Repräsentationsraum, denn Gärten des Hochadels waren traditionell dazu angelegt, die Macht des Fürsten zu demonstrieren. Zugleich wurde mit den gleich- und höherrangigen Adelshäusern um die größten, schönsten und teuersten Gestaltungselemente konkurriert und damit der gewünschte künftige politische Status innerhalb des europäischen Machtgefüges visualisiert. Alles war darauf ausgerichtet, der Lust, das heißt dem Vergnügen der Herrschaften zu dienen. So gab es meist verschiedene Jagdmöglichkeiten, breite Wege für Kutschfahrten und Pavillons, die u.a. auch vor Wind und Wetter schützten.

Zum anderen wurden Gestaltungselemente auch nach dem Aspekt der Prachtdarstellung ausgewählt. Ganz im barocken Überbietungsgestus wurde beispielsweise seitens der hochadeligen Gartenbesitzer all das präferiert, was größer, höher, breiter, länger und in größerer Anzahl vorhanden war. Dazu zählten nicht zuletzt auch aufwändige Orangerien und Obstbaumplantagen ebenso wie besonders lange Alleen und kunstreiche Tempelchen oder Eremitagen. Ob dabei die einzelnen Gartenelemente einem einheitlichen Stil folgten und in der Gesamtheit aller Elemente ein Ganzes ergaben, war dabei tendenziell nachrangig. Wie in frühneuzeitlichen adeligen Wunderkammern wurde all das zum Teil kontrastiv nebeneinander gestellt, was als besonders kostbar und selten galt.

Während also Lustgärten im hochadeligen Kontext vor allem als Repräsentationsraum und Ort herrschaftlicher Vergnügungen genutzt wurden, sahen das die nicht-vornehmen oder auch „,bürgerlichen“ Gartenbesucher des ausgehenden 18. Jahrhunderts ganz anders. Theoretisch müsste im Folgenden auch auf die Gartennutzung im 19. und 20. Jahrhundert eingegangen werden, was jedoch aus Platzgründen unterbleibt. Einen allgemeinen Überblick über den Nutzungswandel historischer Gärten von der Antike bis zur Gegenwart liefert Rohde (2004).

Die Analyse der historischen Parkbeschreibungen des 18. Jahrhunderts zu Park Kassel-Wilhelmshöhe hat ergeben (vgl. Stobbe 2009), dass die damaligen Besucher 
aus einer Vielzahl von Motiven in den Park fuhren oder gingen und darüber wortund kenntnisreich schrieben. So suchten sie nicht nur ästhetisches Kunst- und Naturerleben und eine dadurch hervorgerufene moralische Erbauung oder gar Läuterung. Sondern man ging ebenso gerne in den Park anlässlich besonderer Festivitäten wie zum Beispiel beim Besuch des preußischen Königspaars 1799 in Kassel. Da das junge Königspaar auf Schloss Wilhelmshöhe logierte, sammelte sich dort laut damaligen Zeitungsberichten eine große Menge Schaulustiger. Auch fanden dort zu Ehren der Gäste Wasserspiele und nächtliche Illuminationen statt, wie es seit Generationen bei solchen Anlässen üblich war.

Gerade Wilhelm IX., der von 1785 bis 1821 als Landgraf und ab 1803 als Kurfürst Wilhelm I. in Kassel residierte, gelang es auf geschickte Weise, mehrere Interessen mit seiner Gartenanlage zu bedienen bzw. sowohl seinen Bedürfnissen als auch denen seiner Besucher entgegen zu kommen. So fanden als eine Art Zugeständnis seit Fertigstellung der bis heute erlebbaren Wasserspiele im Jahr 1793 mit den Stationen Kaskade - Steinhöfer Wasserfall - Teufelsbrücke und Plutogrotte Aquädukt - große Fontäne mehrmals im Jahr kostenlos Wasserspiele im Park statt (vgl. Abb. 2). Eingeleitet wurden sie durch ein Schauessen in einem eigens dafür errichteten Pavillon. Wenn die Tafel aufgehoben worden war, begannen auf Geheiß des Fürsten die Wasserspiele. Glaubt man den zeitgenössischen Berichten, waren sie ein kulturelles Highlight und gesellschaftliches Event zugleich.

Immer wieder wird auch davon berichtet, dass Marburger und Göttinger Studenten mit ihren Professoren Fahrten nach Wilhelmshöhe unternahmen - unter anderem, um Kenntnisse über die neu eingeführten Baumarten zu erlangen. In der berühmten Baumschule, die oberhalb der Marstallgebäude rechterhand der Hauptchaussee lag, konnten zudem Setzlinge der neu eingeführten nordamerikanischen Baumarten erworben oder ertauscht werden. Darüber hinaus war ein starkes Motiv für einen Besuch im Park der Wunsch nach einer angenehmen „Landpartie“, ebenso wie ein Parkbesuch ein fester Programmpunkt bei der Besichtigung der Sehenswürdigkeiten einer Stadt war. Die damaligen Reiseführer der Stadt Kassel weisen ausdrücklich darauf hin, ebenso wie es auch um 1800 eigene Parkführer von hofnahen Autoren gab, die bestimmte Routen durch den Park vorgaben und auf das Engagement des Landgrafen in Fragen der Gartenkunst ausdrücklich hinwiesen.

Durch die verschiedenen Gartendarstellungen in Bild und Text wurden also von verschiedenen Interessensgruppen unterschiedliche zielgerichtete Interpretationen der Gartenanlage(n) vorgenommen, die wiederum von späteren Reisenden vor einem Besuch im Park rezipiert wurden. Die jeweiligen Nutzungsansprüche standen dabei im engen Zusammenhang mit den jeweiligen Vorstellungen, Wünschen, Bedürfnissen und Gesellschaftsvorstellungen, die im späten 18. Jahrhundert mit Fragen der Gartenkunst verbunden wurden. Dazu zählten etwa eine patriotische Grundstimmung sowie die Vorstellung von der Veredelung des Menschen durch Kunst. Denn in dem Moment, in dem Gartengestaltung nicht mehr, wie Jahrhunderte lang praktiziert, der Architektur untergeordnet, sondern zur 
obersten Kunst erklärt wurde, konnten bestimmte Vorstellungen aus der Diskussion über das „Bürgerliche Trauerspiel“ auch auf die Gärten übertragen werden. Der Spaziergang in einem Garten konnte dann ähnlich wie der Besuch im Theater eine läuternde Funktion bekommen; Gärten wurden zu ,moralischen Erziehungsanstalten" umfunktioniert.

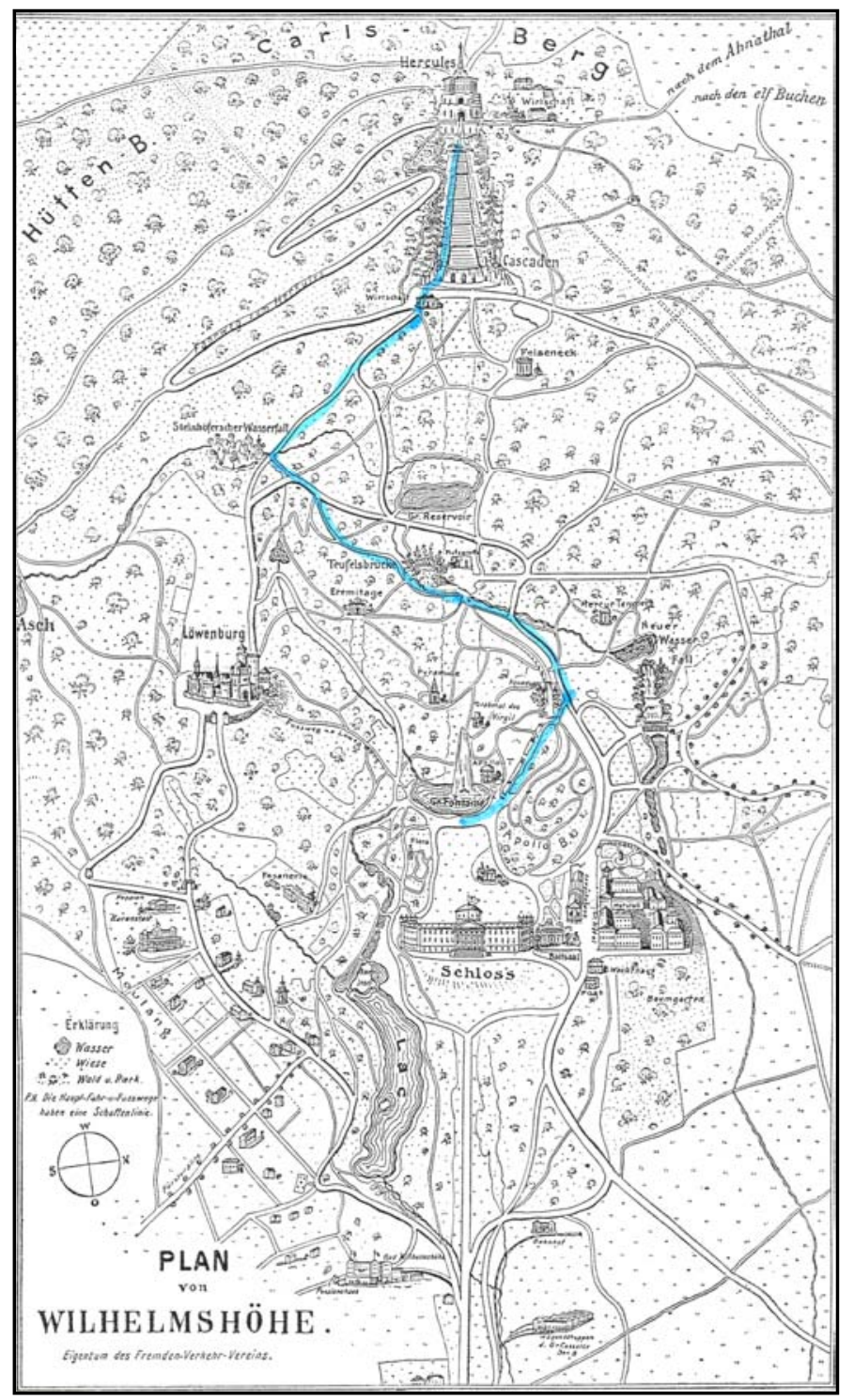

Abb. 2: Auf diesem Überblickplan ist der Weg eingezeichnet, den die Besucher während der Wasserspiele durch den Park nehmen (Quelle: Paetow 1929, Anhang, Bearbeitung: Stobbe) 
Gartenbeschreibungen sind also keinesfalls nur als vergleichsweise neutrale Beschreibungen des Ist-Zustandes zu werten, sondern sie legen Zeugnis von dem Versuch nicht-adeliger Gartenbesucher ab, sich Gärten auf ihre Weise anzueignen. Häufig lässt sich deutlich die Intention herauslesen, die Anlage in einem Sinne zu deuten, der bewusst quer zu der Intention des Fürsten lag. Letztendlich zeigt sich daran aber auch, dass es Diskussionen über die Bedeutung eines historischen Parks nicht erst seit heute gibt und dass die Interessen, die mit der Nutzung dieser Gartenanlagen verbunden sind, auch schon Ende des 18. Jahrhunderts stark differieren konnten.

\section{Visionen einer künftigen Nutzung}

Losgelöst von dem derzeitigen „Boom“ im Gartentourismus-Sektor und abseits merkantiler Interessen ist zu überlegen, wie auch langfristig ein Interesse am Erhalt historischer Gartenanlagen im Bewusstsein der Menschen verankert werden kann. Denn auch wenn bislang noch von steigenden Besucherzahlen und großen wirtschaftlichen Potenzialen ausgegangen wird, ist der Tatsache ins Auge zu sehen, dass diese Art Mode auch stagnieren oder aber von anderen TourismusTrends abgelöst werden kann. Deshalb sollte in allen Tourismuskonzepten rund um den Garten der Aspekt berücksichtigt werden, historische Gärten nachhaltig auch im kulturellen Gedächtnis zu verankern. Oder anders: Die Art der Vermittlung vor Ort entscheidet letztlich auf lange Sicht darüber, ob Gärten auch von den nachfolgenden Generationen erhalten und als schützenswert betrachtet werden.

An diesem Punkt setzt das nach wie vor aktuelle und lesenswerte Werk „Interpreting Our Heritage“ (1957/2007) von Tilden an. Der Gründungsvater des Konzepts der Landschaftsinterpretation vertritt darin die Ansicht, durch sinnvolle Vermittlung zum nachhaltigen und dauerhaften Schutz von Kultur- und Naturgütern zu gelangen (vgl. Kreisel, Reeh u. Gee 2002). Übertragen auf historische Gärten bedeutet dies, sie unter anderem auch deshalb in ihren Besonderheiten bekannt und erlebbar zu machen, damit sich die Menschen mit ihnen identifizieren können. Denn nur so kann der Grundstein dafür gelegt werden, dass historische Gärten über die örtliche Bevölkerung hinaus als „eigene“ Kulturgüter anerkannt und vor einer späteren möglichen Vernachlässigung oder gar Zerstörung bewahrt werden.

Mittlerweile gibt es bereits die ersten, wenn auch noch sehr allgemeinen Studien über die Besuchsbereitschaft und das Besucherverhalten in historischen Gärten (vgl. Brandt 2004). Häufig wird jedoch nur mit Annahmen wie der folgenden operiert: „Das Interesse der typischen Gartenbesucher hat aber den Schwerpunkt auf dem generellen Bedürfnis nach Ruhe, Idylle, Ästhetik und Natur. Die Mehrheit der Besucher will einfach einen erholsamen Tag in attraktiver (!) Umgebung. Sie sind keine Gartenspezialisten, sind oftmals im Zusammenhang mit einem eigenen Garten am Thema Gärtnern interessiert, spüren jedoch unterbewusst die 
hohe ästhetische Qualität und Authentizität sowie das Distanz schaffende Anderssein historischer Gartenanlagen“ (Antz 2007, S. 75, Hervorhebung im Original). Fraglich daran ist, ob es tatsächlich bloß einem ,unterbewussten Spüren“ überlassen bleiben sollte, dass historische Gärten etwas Anderes als normale Grünflächen sind. Viel eher sollte von Anfang an deutlich gemacht werden, dass sich der Besucher nicht in irgendeinem Naherholungsgebiet befindet, sondern an einem Ort, der in einem spezifischen historischen Kontext entstanden ist.

Ausschließlich das angenommene Bedürfnis der Gartenbesucher nach Ruhe, Idylle, Ästhetik und Natur in den Vordergrund zu stellen, macht historische Gartenanlagen ununterscheidbar von Grünflächen jedweder Art. Das kann nicht der Sinn einer Vermittlung historischer Gartenanlagen sein, wie auch die Beiträge im Diskussionsteil des Sammelbands „Marketing für Gärten und Schlösser“ (2004), wenn auch in unterschiedlichen Nuancen und mit jeweils anderen Konsequenzen, betonen. Der Besucher sollte im Sinne Tildens zumindest das Angebot erhalten, die Besonderheiten der jeweiligen Anlage zu erleben, Neugierde und ein Bewusstsein dafür zu entwickeln, wie sich die jeweilige Anlage im Laufe der Jahrhunderte verändert hat, wie sie damals entstanden ist und genutzt wurde. Das Leitkonzept Tildens dabei lautet: „Through interpretation, understanding; through unterstanding, appreciation; through appreciation, protection“" (Tilden 1957/2007, S. 65).

Historische Gartenanlagen, wie überhaupt jede Form historischer Denkmäler, können und sollten nicht beliebig in derzeit im Kulturbetrieb favorisierte Nutzungskonzepte integriert werden, ohne - wie bisher - ein konkretes Besucherleitkonzept zur Vermittlung der Einzigartigkeit der jeweiligen Anlage entwickelt und diskutiert zu haben. Der Transfer bestimmter Nutzungskonzepte muss den örtlichen und regionalen Gegebenheiten angepasst und nötigenfalls modifiziert werden. Dabei ist ein behutsamer Umgang mit der historischen Bausubstanz und den Pflanzenarten zwingend erforderlich. Das bedeutet jedoch nicht, sich jeder Form von Veränderung und Nutzung zu verschließen, sondern stattdessen eine gewisse Form der Gelassenheit zu wahren. Denn Gärten sind per se durch Jahreszeiten und Wachstumsphasen einem gewissen natürlichen Wandel unterworfen; sie haben in der Regel die Fähigkeit zur Rekreation bei kurzfristigen Störungen. Vielmehr sind konkrete Zielvorstellungen zu formulieren, was ein Besucher potenziell von dem Parkbesuch „mitnehmen“ soll.

Dabei ist die Balance zu wahren zwischen Information über das, was zu sehen ist, und dem, was es auf eigene Initiative hin zu entdecken gibt und mit Bekanntem verknüpft werden kann. Das besondere Kunst- und/oder Naturerleben im Park lässt sich nicht auf den Punkt genau verordnen; es können lediglich Hinweise gegeben werden, wie diese Anlage aus damaliger Sicht konzipiert worden ist. Welche Funktionen nahm die Anlage damals ein, wie wurden die dort befindlichen Gestaltungselemente bildlich und textlich dargestellt? Was ist davon noch heute zu erleben? Ziel der Landschaftsinterpretation ist es, nicht eindeutige Lesarten oder Interpretationen vorzugeben, sondern dem Besucher individuell gestaltete Aneignungsmöglichkeiten anzubieten. Das kann zum Beispiel auch bedeuten, 
verschiedene Routen anzubieten, die je nach Interesse und Neigung individuell ausgewählt werden können.

Zu überlegen wäre, ob nicht eine erlebnisorientierte Nutzung von Geschichte angestrebt werden kann, bei der wohl dosiert Elemente einer gehobenen Eventkultur aufgegriffen als auch Wissen auf dem aktuellen Stand der Forschung vermittelt wird. Der Park würde dabei als ein begehbarer und erlebbarer Ort der damaligen Gartenkultur inszeniert werden. Mit der Einrichtung eines speziellen Parkmuseums könnte Kassel-Wilhelmshöhe unabhängig von einer möglichen Nobilitierung als Unesco-Weltkulturerbe aufgewertet werden. Denn bislang gibt es zwar seit dem Jahr 2000 ein allgemeines Gartenkunst-Museum in Schloss Fantaisie bei Bayreuth. Auch befindet sich im Benrather Schloss in Düsseldorf das Museum für Europäische Gartenkunst; ein Hofgärtnermuseum wurde in Glienicke (Potsdam) eingerichtet, indem die Sonderausstellung „Preußisch Grün“ (2004) im Jahr 2006 in eine Dauerausstellung umgewandelt wurde. Als richtungsweisend kann in diesem Zusammenhang die Dauerausstellung zu Fürst Pückler im Muskauer Schloss angesehen werden, da sie genau diesen Ansatz verfolgt, neue Wege der Parkvermittlung zu beschreiten.

\section{Ausblick}

Am Beispiel des Bergparks Kassel-Wilhelmshöhe lässt sich zeigen, dass historische Gartenanlagen sowohl in ihrer Gestaltung als auch in der Nutzung einem wenn auch langsamen, so doch stetigen - Wandel unterworfen sind. Beide Aspekte waren damals wie heute zeitspezifischen Konventionen unterworfen, wobei die ehemaligen Erbauer und Gartenbesitzer ebenso wie die heute zuständigen Institutionen wesentlichen Einfluss auf die jeweiligen Deutungen genommen haben. Waren hochadelige Gartenanlagen wie Kassel-Wilhelmshöhe traditionell höfische Repräsentationsräume, so erhalten sie in den letzten Jahren im Bereich des Stadtmarketings immer größere Bedeutung.

Wie die Zukunft dieser Gartenanlage aussieht, ist derzeit noch ungewiss. Anfang 2010 wird der Antrag bei der Unesco eingereicht, die Entscheidung ist im Jahr 2011 zu erwarten. Dem Antrag werden die Forschungsergebnisse der Tagung „Hortus ex machina. Der Bergpark Wilhelmshöhe im Dreiklang von Kunst, Natur und Technik“ vom Mai 2009 in Kassel beigefügt. In der jüngsten Podiumsdiskussion im Februar 2009 war bereits der Konsens in allen Redebeiträgen greifbar, dass die Aufnahme des Parks Kassel-Wilhelmshöhe in die Liste des Weltkulturerbes einem Anstieg der Besucherzahlen und damit einer Mehreinnahme in unterschiedlichen Finanzsegmenten der Stadt Kassel nur förderlich sein kann. Die zur Zeit in der Ausarbeitung befindliche Nordhessen-Tour wird für den notwendigen Bekanntheitsgrad sorgen. Was jedoch nach wie vor zu fehlen scheint, ist ein durchdachtes Besucherleitkonzept für den Garten selbst. 


\section{Literatur und Quellen}

Antz, C. (2007): „Gartenträume“ - Kulturräume wandeln sich in Sachsen-Anhalt zu Tourismusangeboten. In: Brandt, A., Bothmer, W. v. u. Mangels, C. (Hrsg.): GartenNetze Deutschland. Entwicklung - Vernetzung - Vermarktung historischer Gärten. Rostock. S. 61-75.

Brandt, A., Bothmer, W. v. u. Mangels, C. (Hrsg.) (2007): GartenNetze Deutschland. Entwicklung - Vernetzung - Vermarktung historischer Gärten. Rostock.

Brandt, A., Bothmer, W. v. u. Rohde, M. (Hrsg.) (2004): Marketing für Gärten und Schlösser. Touristische Nutzungskonzepte für Gärten, Parks, Herrenhäuser und Schlösser. 2. Aufl., Rostock.

Brandt, A. (2004): Besuchsbereitschaft und Besucherverhalten in Gärten und Schlössern - Ergebnisse der Marktforschung. In: Brandt, A., Bothmer, W. v. u. Mangels, C. (Hrsg.): GartenNetze Deutschland. Entwicklung - Vernetzung - Vermarktung historischer Gärten. Rostock. S. 16-26.

Gutachten Marketing, DMG Besucherstatistik (2004): Neuordnung der Museumslandschaft Kassel. Präsentation für den 07. Mai 2004. Siehe Homepage des Hessischen Ministeriums für Wissenschaft und Kunst Wiesbaden: www.hmwk.hessen.de; unter: Kultur - Kulturpolitik - Museumslandschaft Kassel - Gutachten.

Hlavac, C. (2005): Touristische Ressource „Landschaft“ am Beispiel von Gärten und Parks. In: ZELTSchriften. Zeitschrift für Landschaftsinterpretation und Tourismus 4.1, S. 4-7.

Klaffke, K. (2007): Gartennetzwerk Deutschland - eine Stellungnahme. In: Brandt, A., Bothmer, W. v. u. Mangels, C. (Hrsg.): GartenNetze Deutschland. Entwicklung - Vernetzung - Vermarktung historischer Gärten. Rostock. S. 45-51.

Koseck, G. u. Schlosser, P. (2008): Auftaktsymposium Mai. Gartennetzwerk Gartenregion. In: Wirtschaft Nordhessen 5, S. 24.

Kreisel, W., Reeh, T. u. Gee, K. (2002): Landschaftsinterpretation: durch Erlebnis, Verständnis und Bewusstseinsbildung zum nachhaltigen Tourismus. In:

ZELTSchriften. Zeitschrift für Landschaftsinterpretation und Tourismus 1.1, S. 5-7.

Paetow, K. (1929): Klassizismus und Romantik auf Wilhelmshöhe. Kassel.

Ringkamp, C. (2007): Bundesweite Initiative Gartennetz Deutschland. In: Brandt, A., Bothmer, W. v. u. Mangels, C. (Hrsg.): GartenNetze Deutschland. Entwicklung - Vernetzung - Vermarktung historischer Gärten. Rostock. S. 5255. 
Rohde, M. (2004): Nutzungswandel in historischen Gärten und Empfehlungen aus Sicht der Denkmalpflege. In: Brandt, A., Bothmer, W. v. u. Rohde, M. (Hrsg.) (2004): Marketing für Gärten und Schlösser. Touristische Nutzungskonzepte für Gärten, Parks, Herrenhäuser und Schlösser. 2. Aufl., Rostock. S. 28-36.

Spanjer, J. (2007): Europäisches Gartennetzwerk EGHN European Garden Heritage Network. In: Brandt, A., Bothmer, W. v. u. Mangels, C. (Hrsg.): GartenNetze Deutschland. Entwicklung - Vernetzung - Vermarktung historischer Gärten. Rostock. S. 31-35.

Stobbe, U. (2009): Kassel-Wilhelmshöhe. Ein hochadeliger Lustgarten im 18. Jahrhundert. (Erscheint Herbst 2009 im Deutschen Kunstverlag, München).

Tilden, F. (1957/2007): Interpreting Our Heritage. Principles and Practices for Visitor Services in Parks, Museums, and Historic Places. New York, 4. Auflage.

Unesco (1972): Übereinkommen zum Schutz des Kultur- und Naturerbes der Welt.

http1: http://www.hmwk.hessen.de <02.03.2009> http2: http://www.museum-kassel.de $<17.05 .2009>$ http3: http://www.welterbe-kassel.de <17.05.2009> http4: http://www.kultur-baut-auf.de <17.05.2009> http5: http://www.eghn.org <17.02.2009> http6: http://www.ihk-kassel.de/welterbe <17.05.2009> 



\section{Kriegsbewirtschaftung als Faktor eines Landnut- zungswandels - Das Beispiel der Eichenschälwäl- der vor und während des Ersten Weltkrieges}

Axel Bader*

* Ich danke den beiden anonymen Gutachtern für ihre hilfreichen Kommentare und Anmerkungen zu einer früheren Version des Artikels. 


\begin{abstract}
This historical essay's aim is to introduce war as a reason for a land use change into the scientific discussion dominated by geographers and landscape planners. Case studying German tannin bark production in oak coppice around World War I, a significant land use change can be detected. From 1850ies to 1890ies coppice was important for the tanning industry. Therefore it had the highest ground rent in forestry and covered 450.000 ha in 1890. Import of Argentine tannin put the German coppice under pressure. In 1913 state owned forestry had hardly any coppice under its rule anymore. The state had switched to timber. Rests of coppice still existed but were owned by farmers. Lacking financial substitutes for a switch, farmers employed an early form of multi-use forestry. The war suspended any import so the tanners had to switch back to German resources. 1915 and 1916 tannin prices rose but the powerful tanners could set price caps. Coppice owners now were wooed financially and ideologically. Overexploitation of coppice, new techniques and an easier access led to a use of tannin wood from 1916 on. The reenactment of tannin bark production in oak coppice was extensive but short lived.
\end{abstract}

\title{
Einleitung
}

Thema, Leitfrage, Forschungsstand

Als Landnutzung wird die Art der Inanspruchnahme von Landflächen und Böden durch den Menschen bezeichnet. Die Nutzung einer Bodenfläche hängt von den natürlichen Gegebenheiten wie vom Interesse und den Fähigkeiten der potentiellen Nutzer ab. Alle drei Bedingungen können sich mit der Zeit ändern. „Landnutzungs-Wandel“" als Analysekonzept lenkt deshalb den Blick auf die Änderung dieser Inanspruchnahme im Laufe der Zeit. Die einschlägigen Arbeiten zum Landnutzungswandel wurden meist von Geographen, Forst- und Agrarwissenschaftlern verfasst (vgl. Denecke 2005, S. 17; Konold 1996, S. 123; Bub 2003; Geldmacher 2006). Sie machten wirtschaftliche Erfordernisse, kulturelle, technische und chemische Entwicklungen, Naturkatastrophen, klimatische Änderungen und in jüngster Zeit auch Subventionen als treibende Kräfte eines Landnutzungswandels aus (vgl. Bürgi 2009 in diesem Band; Rau 2005; Kümmerle 2007). In jüngster Zeit befassten sich auch Landschaftsplaner und -ökologen mit dem Thema (Palang 2002), was für ein zunehmendes multidisziplinäres Interesse für das Themenfeld spricht. Eine geschichtswissenschaftliche Befassung mit dem Thema liegt aber bisher nicht vor, was schon Corvol und Amat (1994) anmerkten. 
Kriege als treibende Faktoren des Landnutzungswandels blieben bisher weitgehend unbeachtet ${ }^{1}$. Das überrascht, denn die Veränderungen zu Kriegszeiten waren einschneidend. Das soll hier am Beispiel der Gerbrindennutzung im Niederwaldbetrieb zur Zeit des Ersten Weltkriegs gezeigt werden.

\section{Methode}

Durch die Verortung des Autors im Feld der Forstgeschichte ergibt sich eine Methodenauswahl, die sich eng auf die in der Geschichtswissenschaft übliche hermeneutische Herangehensweise bezieht. Durch ein Interpretieren der vorliegenden Quellenmaterialien soll das Handeln der Menschen verstehbar gemacht werden. Der Quellenbestand erlaubt eine Verschränkung von Mikro- und Makroebene, denn die Akten der Kriegsleder AG, die auszuwertenden Statistiken und die Preislisten beziehen sich meist auf einzelne Bundesstaaten oder sogar das gesamte Reichsgebiet. Dagegen erlaubt der Bericht über das Forstamt Kirchenlamitz einen Blick auf die Situation vor Ort zu werfen. In der Zusammenschau ergibt sich so ein umfassendes Bild, in dem gesamtstaatliche Problemstellungen und Entwicklungen auch auf der kleinsten Ebene - dem einzelnen Forstamt - ihren Niederschlag finden. Diese Verschränkung der Ebenen lässt es zu, die gewonnenen Erkenntnisse in ihrer Übertragbarkeit auf andere Örtlichkeiten besser einzuschätzen, obwohl eine Repräsentativität im Sinne heutiger sozialwissenschaftlicher Forschung nicht erreicht werden kann.

\section{Fallbeispiel}

Die Wahl eines räumlichen Ausschnitts fiel auf die Region Fichtelgebirge. Das Forstamt Kirchenlamitz liegt zwischen Bayreuth und Hof etwa $15 \mathrm{~km}$ von der deutsch-tschechischen Grenze entfernt. Kirchenlamitz wurde ausgewählt, weil von dort Berichte eines Försters überliefert sind, die einen umfassenden Überblick über die örtliche Gerbrindenbewirtschaftung vor und während des Krieges zulassen.

Der Aufstieg der örtlichen Gerbereiwirtschaft hing eng mit den naturräumlichen Besonderheiten des Fichtelgebirges zusammen. Das benötigte Rohmaterial konnte in der nächsten Umgebung gewonnen werden. Häute lieferten die Metzgereien, Gerbrinde der umliegende Wald. Daneben bedurfte es noch geeigneten Wassers. Gerbsäure und kohlensaurer Kalk gehen unlösliche Verbindungen ein. Wird hartes Wasser zum Gerben verwendet, bedeutet das deshalb immer einen gewissen Gerbstoffverlust. Die Flüsse und Bäche des Fichtelgebirges führten weiches Wasser. Sie waren deswegen gut für die Gerberei geeignet. So bestanden

\footnotetext{
${ }^{1}$ In vielen Arbeiten werden der Dreißigjährige Krieg, die Revolutionskriege sowie die beiden Weltkriege pauschal als Faktoren eines Landschaftswandels oder Landnutzungswandels angenommen (vgl. Thormann 2002, S. 55). Eine Auseinandersetzung mit den tieferen Ursachen unterbleibt meist. So wird ein Krieg zwar als Epochenschwelle wahrgenommen, aber nicht im engeren Sinn als Faktor des Landnutzungswandels.
} 
allgemein gute örtliche Voraussetzungen für die Entstehung eines regionalen Gerberzentrums an den Flussläufen von Eger, Saale und ihren diversen Nebenflüssen. Als ab der ersten Hälfte des 19. Jahrhunderts wegen der fortschreitenden Industrialisierung immer mehr Leder benötigt wurde, stieg die Lederproduktion auf dem gesamten Gebiet des späteren Deutschen Reiches stark an (vgl. Gebek 1935). Anfang des 19. Jahrhunderts bestanden 65 Gerbereien in dem Gebiet des Oberforstamtes Selb, darunter zwei in Kirchenlamitz direkt. Das Gerbereihandwerk hatte sich zu einer Zeit entfaltet, als lediglich lokale Bedürfnisse zu befriedigen waren. Die Ledererzeugung diente vor allem zur Versorgung der örtlich ansässigen Schuster und Sattler (vgl. Eisele 1918).

\section{Gliederung}

Die Darstellung beginnt mit einem einführenden Teil. Hier stehen die forstwissenschaftlichen Grundlagen der Schälwaldwirtschaft im Mittelpunkt. Sie sind heute auch in forstlich interessierten Kreisen weitgehend unbekannt. Daran schließt sich eine Darstellung der Entwicklung bis zum Beginn des Ersten Weltkriegs 1914 an. Es werden die Gründe für das Wachsen und den Niedergang der Schälwaldwirtschaft als Landnutzungsform erörtert. Das Wiederentdecken der Rindennutzung in der Zeit des Ersten Weltkrieges steht im Mittelpunkt des dritten Kapitels. Ein Ausblick auf die Nachkriegszeit und ein Fazit beschließen den vorliegenden Aufsatz.

\section{Quellen}

Die Entwicklung der Eichenschälwaldwirtschaft konnte mithilfe einer Auswertung der entsprechenden Jahrgänge der forstlichen Fachzeitschriften Forstwissenschaftliches Centralblatt (FWC), Silva und Allgemeine Forst- und Jagdzeitscbrift (AFJZ) nachvollzogen werden. Neben Fachartikeln wurden hier Kommentare zur Marktentwicklung für Forstprodukte, Preislisten und Versteigerungen veröffentlicht. Staatliche Maßnahmen wie Markteingriffe, Subventionen, Verordnungen und Gesetze wurden ebenfalls kommentiert und für die Leserschaft - Angehörige des Forstsektors - aufbereitet. Die einzelnen Hefte sind zu Jahresbänden zusammengefasst und fortlaufend durchnummeriert. Zum Fallbeispiel Kirchenlamitz im Fichtelgebirge liegen Berichte des Forstassessors Eisele vor. Eisele leistete seinen Dienst im Forstamt Kirchenlamitz ab und berichtete im Forstwissenschaftlichen Centralblatt über die dortigen Revierverhältnisse. Als auszuwertende Quellen konnte auf die Akten der Kriegsleder AG aus dem Bundesarchiv Berlin und des Forstamts Rumbeck aus dem nordrhein-westfälischen Landesarchiv Münster zurückgegriffen werden. 


\section{Forstwissenschaftliche Grundlagen}

Viele Baumrinden enthalten Gerbstoffe. Nutzt man die Gerbeigenschaften einer Rinde aus, so bezeichnet man sie als ,Lohrinde‘. Unter den heimischen Bäumen sind die Rinden der Kastanie, Eiche und Fichte besonders gerbstoffhaltig, deswegen bildete sich vor allem bei der Eiche neben der Holznutzung als ,zweite Hauptnutzung die Gerbrindenproduktion heraus (vgl. Endres 1922, S. 820). Das Holz aus diesen Schälwäldern ist eigentlich ein Abfallprodukt und wird vor allem als Brennholz genutzt (vgl. Paeßler 1917, S. 398). In Weinbaugebieten an Rhein und Mosel war auch die Nutzung der dünnen Stangen als Rankhilfe für Reben üblich (vgl. Paeßler 1917, S. 395; Ostermann 2002, S. 36f., 44f.).

\section{Niederwaldwirtschaft}

Die Niederwaldwirtschaft ist eine historische Form der Forstwirtschaft, die in jüngster Zeit von Kurzumtriebsplantagen kopiert wird. Die verwendete Baumart muss dabei zu einer Regeneration aus Stockausschlägen fähig sein. Die ganze Schälwaldfläche wurde dabei alle 15 bis 20 Jahre abgeerntet, ohne dass neue Bäume gepflanzt wurden. Die Verjüngung erfolgt im Niederwald ausschließlich aus diesem Stockausschlag. Eine besondere Bestandspflege war dazu nicht nötig (vgl. Bauer et al. 2006, S. 59).

Wurden die frisch gehauenen Schläge landwirtschaftlich genutzt bis die Stockausschläge zu hoch waren, so bezeichnete man es als Röderwirtschaft. Diese Art der Anschlussnutzung war sehr arbeitsaufwendig und laugte den Boden stark aus. Deshalb wurde sie im Laufe des 19. Jahrhunderts weitgehend verboten. Örtliche Ausnahmen waren die Siegener Hauberge, in denen die Röderwirtschaft eine bedeutende Rolle spielte (vgl. Pott 1985).

Eine Gerbstoffproduktion im Niederwaldbetrieb wurde nur mit der Eiche betrieben. Der Gerbstoffgehalt der Eichenrinde ist dann am höchsten, wenn sie noch nicht allzu rissig ist. Das ist bei Rinden von jungen Bäumen der Fall. Schälwälder gab es in den meisten deutschen Waldgebieten, besonders jedoch in der Eifel, dem Hunsrück und dem Fichtelgebirge (vgl. Paeßler 1917, S. 394).

Neben der Eichen- spielte auch noch die Fichtenrinde eine gewisse Rolle. Der forsttechnische Unterschied zwischen Eichen- und Fichtenrindennutzung bestand darin, dass die Lohrindennutzung bei der Eiche eine Hauptnutzung, bei der Fichte aber nur eine Nebennutzung darstellte. Fichten wurden auch dann entrindet, wenn ihre Rinde nicht als Gerberlohe verwendet wurde. Die Rinde verblieb dann einfach als Dünger im Wald. In einigen Gebieten ist die Nutzung der Lohe bereits im Frühmittelalter nachgewiesen. Die Blütezeit der Lohwirtschaft lag etwa zwischen 1840 und 1880. Mit der Industrialisierung stieg die Nachfrage nach Leder sprunghaft an. Das alles führte dazu, dass Mitte des 19. Jahrhunderts die Lederindustrie zum drittgrößten Gewerbezweig im Gebiet des späteren Deutschen Reiches geworden war (vgl. Endres 1922, S. 824). 
Mit der steigenden Nachfrage nach Leder und damit nach Gerbstoffen wurden im 18. und 19. Jahrhundert viele Buchenwälder in Eichenschälwälder umgewandelt. Man pflanzte vor allem Stieleichen, die es bis dahin im Schiefergebirge nur selten gegeben hatte.

Um 1890 wurden in Deutschland etwa 445.000 ha Schälwald genutzt (vgl. Endres 1922, S. 821). Eine so große Flächenausdehnung wurde nie mehr erreicht. Die Lohwirtschaft brachte für die Waldbesitzer zusätzliche Einkommensmöglichkeiten mit sich. Für die Kleinbauern ergab sich die Möglichkeit, ihre kleinen Waldflächen kontinuierlich zu nutzen, mit der Lohe zusätzliches Geld zu verdienen und für sich selbst Brennholz, und auf den abgeernteten Flächen Roggen, Buchweizen und Streu zu produzieren. Die Arbeit in den Lohwäldern konnte außerdem gut in den jährlichen Arbeitsrhythmus eingebaut werden. Sie fiel zwischen die Bestellung der Felder im Frühjahr und die Heuernte. Größere Waldbesitzer vergaben die Arbeit häufig an Tagelöhner. Als Bezahlung erhielten diese meist das Holz sowie das Recht, im ersten und zweiten Jahr nach dem Abholzen Getreide einzusäen. Außerdem sicherten die Lohmühlen und Gerbereien Arbeitsplätze. So entwickelten sich z. B. Neuerburg in der Eifel und Wiltz in Luxemburg zu regelrechten Gerberstädten.

Nach 1880 begannen die Preise für Eichenlohe kontinuierlich zu fallen (vgl. Forstwissenschaftliches Centralblatt 1896, S. 643). Ursachen waren einerseits technische Neuerungen und Veränderungen bei den Gerbereien wie die Einführung der Extraktgerbung, die zunehmenden Einfuhren von Gerbstoffen aus Übersee und die Entwicklung synthetischer Gerbstoffe, andererseits der einsetzende Strukturwandel in der Landwirtschaft (z.B. durch den Einsatz der Thomasschlacke aus der Stahlproduktion als Kunstdünger), aber auch die Verdrängung von Brennholz durch Kohle und später Heizöl. Dennoch wurden, vor allem in Gebieten, in denen es wenig industrielle Arbeitsplätze gab, die Lohhecken noch über Jahrzehnte in relativ großem Umfang genutzt.

\section{Lohen}

Die Hiebszeit in den Eichenniederwäldern fiel ins Frühjahr, weil sich die Rinde am besten ablösen lässt, wenn der Saft zu ,steigen` beginnt. Die gerbstoffhaltige Rinde wurde vor dem Laubaustrieb von den frisch eingeschlagenen Eichenstangen mit dem Lohlöffel abgelöst. Die Rindenstücke wurden dann zum Trocknen aufgestellt. Wertsteigernd war eine möglichst schnelle Trocknung der Gerbrinde, weshalb das Wetter eine große Rolle spielte. Die getrockneten Rinden wurden dann in einer Lohmühle gemahlen und dann das Rindenmehl in Wasser eingeweicht. Den entstehende Sud bezeichnete man als Gerbstoffextrakt oder -brühe.

\section{Gerbprozess}

Beim Gerben wird eine Tierhaut in Leder umgewandelt. Jede Tierhaut besteht aus den drei Lagen Ober-, Leder- und Unterhaut (Epidermis, Dermis und Subkutis). 
Bei der Lederherstellung werden vor der eigentlichen Gerbung Epidermis und Subkutis mechanisch entfernt und nur die mittlere Lederhaut, die Dermis weiterverarbeitet. Diese besteht zu einem Drittel aus Eiweiß und Wasser. Wird die Lederhaut in die Gerbbrühe eingelegt, kommt sie in Kontakt mit dem Gerbstoff und das Eiweiß der Dermis verbindet sich mit diesem. Aus dem Eiweiß und dem Gerbstoff entsteht das fertige Leder (vgl. http1).

\section{Die Globalisierung schreitet voran - die Schälwaldwirt- schaft geht zurück}

\section{Die Situation auf dem Lande: Kirchenlamitz am Fichtelgebirge}

Die Ledererzeugung dieser Gerbereien war bis zur Mitte des 19. Jahrhunderts fast ausschließlich für den Bedarf der näheren Umgebung bestimmt. Nur die Betriebe in Hof beteiligten sich an einem überregionalen Handel. Der örtlich ansässige Revierförster beschrieb die Situation folgendermaßen: Die Rotgerber der Umgegend seien durchgehend Ökonomiebesitzer, also Bauern, und betrieben ihre Profession nur nebenbei. Sie produzierten nur soviel, als sie auf den umliegenden Märkten absetzen zu können glaubten. Größere Märkte, wie die Leipziger Messe wären nur wenig besucht (vgl. Eisele 1918, S. 202). Die Betriebsführung im Nebenerwerb erlaubte es, die Produktion dem augenblicklichen Bedarf ohne all zu große Einkommenseinbußen anzupassen. Beim späteren Niedergang des Handwerks sollte sich diese Verbindung noch positiv bemerkbar machen.

Bei einem Gerbrindenverbrauch von 80 bis 200 Zentner und einer Erzeugung von etwa 16 bis 40 Zentner Leder pro Gerberei im Jahre 1860 konnte man nur von Kleinbetrieben sprechen. Es waren allerdings 65 an der Zahl und deswegen war der Lohrindenverbrauch - der nicht allein von den Staatswäldern befriedigt werden musste, aber nur dort heute noch nachweisbar ist - kein geringer. Um 1860 wurden im Staatswald etwa 2400 Zentner Lohrinde geschält. Das war eine deutliche Steigerung dessen gewesen, was noch in den davorliegenden Jahrzehnten geschält worden war. In den 1840er Jahren waren durchschnittlich 240 Zentner, in den 1850er Jahren 320 Zentner ausgewiesen worden.

Die Steigerung der Rindenproduktion stellte die Forstverwaltung vor nicht geringe Schwierigkeiten. Viele Brennholzberechtigte - Pfarrer, Lehrer, Beamte und andere Versorgungsberechtigte bekamen eine festgelegte Menge Brennholz pro Jahr - klagten über einen Qualitätsverlust ihrer Marge. Das war nicht weiter verwunderlich, denn das beste Brennholz wird im Winter geschlagen, wenn das Holz trocken ist, die Rinde aber lässt sich nur dann ablösen, wenn der Saft zu steigen beginnt. Das wiederum ist erst im Frühjahr nach der Blütezeit der Fall. Brennholzund Rindennutzung schloss sich nicht gegenseitig aus, eine Nutzungsart verringerte aber den möglichen Wert der durch die andere erzielt werden konnte. Ein forstverwaltungsinternes Problem kam noch hinzu: Die Schälzeit fiel genau in die 
Zeit, in der eigentlich ein Jahresabschluss zu machen wäre. Man kann also davon ausgehen, dass es lokal auch Stimmen gab, die später der Schälwaldwirtschaft nicht besonders wehmütig nachrufen würden.

Ab 1861 sank die bereitgestellte Rindenmenge wieder langsam, um zwischen 1868 und 1876 ganz wegzufallen. 1877 wurde noch einmal eine größere Menge Rinde ausgeboten, die aber keinen Abnehmer mehr fand. Von da an lässt sich bis 1916 in den Staatswäldern keine Rindennutzung mehr nachweisen.

Die Entwicklungen im Gerbereihandwerk

Die Entwicklung des Gerberhandwerks spiegelt sich in diesen Zahlen. Bis etwa zur Mitte des 19. Jahrhunderts stieg die Zahl der Gerbereien stark an, dann sank sie ebenso schnell wieder ab in den folgenden Jahrzehnten. Die Gründe hierfür sind nicht ganz untypisch für die Zeit der Industrialisierung: Familienbetriebe wurden immer mehr von rationeller produzierenden Fabriken abgelöst, die per Schnellgerbung in rotierenden Gerbfässern der Gerbgrubentechnik weit überlegen waren (vgl. Endres 1922, S. 823). Die Schnellgerberei wiederum war auf Gerbstoffextrakte angewiesen, wie sie vergleichsweise billig aus Südamerika importiert werden konnten.

Das Schnellgerbverfahren war zwar schon in der Zeit der französischen Revolution von Séguin entdeckt worden. In Deutschland wurde es jedoch erst knapp Ende der 1850er Jahre bekannt. Bis es sich auch in ländlichen Gebieten durchgesetzt hatte, verging noch eine lange Zeit, denn große Investitionen waren vonnöten, um eine solche Anlage zu installieren. Wegen des großen Bedarfs an Gerbstoffextrakten beschränkten sich die möglichen Standorte auf die Nähe zu Extraktfabriken und Bahnlinien oder Kanälen. Die Extraktfabriken wiederum siedelten sich um die großen norddeutschen Häfen an, wo eben der Gerbrohstoff aus Amerika angelandet wurde.

\section{Steigerung des Warenverkehrs: Der Import von Quebracho stieg}

Ab 1870 wuchs der Gerbstoffimport immer stärker an. Haupteinfuhrstoff war das Holz und der Holzextrakt des Quebrachobaums. Der Quebrachobaum wuchs in Südamerika. Argentinien war das größte Ausfuhrland. Daneben exportierten noch Brasilien, Paraguay, Bolivien und Uruguay Quebrachoholz. Der Name führt auf die große Härte des Holzes zurück. Er bedeutet ,Axtbrecher ${ }^{{ }^{2}}$. Die spezifische Dichte des Holzes ist so hoch, dass es nicht im Wasser schwimmt. Es wiegt 1250 $\mathrm{kg}$ pro Kubikmeter. Die nach Europa verschifften Stammstücke wurden als, Rollizos' bezeichnet. Daneben wurde auch Quebrachoextrakt exportiert. Wertvoll war vor allem das Kernholz des Baumes, denn es enthält durchschnittlich 20 bis $22 \%$ Gerbstoffe. Der Gerbstoffanteil der Rinde (6 bis $8 \%$ ) und des Splintholzes (2 bis $3 \%$ war demgegenüber geringer. Der Gerbstoffgehalt des Kernholzes konnte

\footnotetext{
${ }^{2}$ Span. quebrar $=$ brechen; el hacha $=$ die Axt.
} 
durch Extraktion fast restlos gewonnen werden. Da das ausgelaugte Holz zudem gutes Brennmaterial abgab, konnten Extraktfabriken meist ohne zusätzliche Brennstoffzufuhr arbeiten.

Der Preis der Rohgerbstoffe hing direkt mit ihrem Gerbstoffgehalt zusammen und Tabelle 1 zeigt deutlich, welche Konkurrenz der heimischen Schälwirtschaft durch den Quebrachoimport erwachsen war. Eichen- und Fichtenrinde waren gegen die exotischen Gerbstoffe chancenlos.

Tab. 1: Gerbstoffanteile

\begin{tabular}{ll}
\hline Eichenrinde & $10-12 \%$ \\
\hline Eichenholz & $3,5-5 \%$ \\
\hline Eichenholzextrakt & $24 \%$ \\
\hline Fichtenrinde & $10,5 \%$ \\
\hline Fichtenrindenextrakt & $21 \%$ \\
\hline Kastanienholzextrakt & $24 \%$ \\
Quebrachoholz & $20-22 \%$ \\
\hline Quebrachoholzextrakt & $63-69 \%$ \\
\hline Mangrovenrinde & $34 \%$ \\
Galläpfel & $30 \%$ \\
Weidenrinde & $5 \%$ \\
\hline
\end{tabular}

Der europäische Markt wurde auf der Weltausstellung in Paris 1867 auf Quebracho aufmerksam. Die deutsche Außenhandelsstatistik wies 1885 erstmalig eine Einfuhr von 5304 t nach (vgl. Endres 1922, S. 822). Vorher spielte es ebenfalls schon eine gewisse Rolle, tauchte in den Statistiken aber nur unter , andere Gerbmittel“ auf. Ab dem Jahre 1888 steigerte sich der Import in das deutsche Zollgebiet kontinuierlich und im letzten Vorkriegsjahr wurden insgesamt $112.284 \mathrm{t}$ Quebrachoholz und 17.278 t Holzextrakt eingeführt (vgl. Endres 1922, S. 823). 


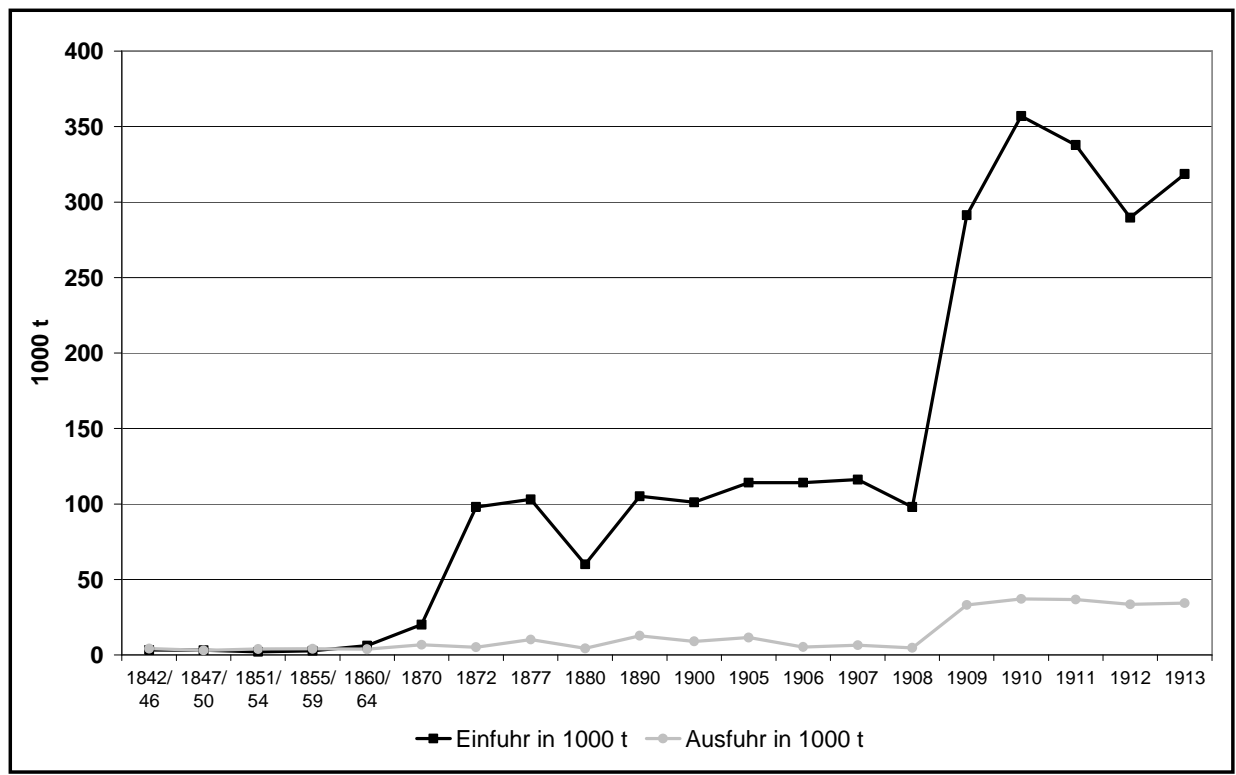

Abb. 2: Entwicklung der Einfuhr und Ausfuhr von Holzborke und Gerberlohe im deutschen Zollgebiet (Eigene Darstellung, Datengrundlage: Endres 1921, S. 828).

Diese Entwicklung müsste sich eigentlich in verstärkten politischen Maßnahmen zum Schutz der Schälwaldwirtschaft niederschlagen. An dieser Stelle sei nur erwähnt, dass fast die gesamte (globale) Wertschöpfungskette ebenfalls in deutscher Hand war. Quebracho wuchs auf Haciendas, die deutschen Auswanderern oder deutschen Firmen gehörten. Die Extraktfabriken in Argentinien selbst wurden von deutschen Firmen betrieben, der Extrakt dann von deutschen Reedereien nach Hamburg oder Bremen transportiert. Transportierte man nur die ,Rollizos', wurde es in Extraktfabriken, die sich um die deutschen Überseehäfen angesiedelt hatten, weiterverarbeitet. Der Verkehr wurde weitgehend in Reichsmark abgewickelt, so dass theoretisch mögliche Devisenverluste ebenfalls nicht anfielen.

Stetig fallende Rindenpreise (vgl. FWC 1896, S. 615), bei Versteigerungen reihenweise zurückbleibende Rindenmengen (vgl. FWC 1896, S. 643) und neue Gerbtechniken, die ganz ohne Rinde auskamen, ließen ab 1890/95 keine günstige Marktentwicklung mehr erwarten.

\section{Das Fallbeispiel Kirchenlamitz}

Die Zahl der Betriebe hatte sich unter diesen Bedingungen auch in der untersuchten Region am Fichtelgebirge stark verringert. In Hof bestanden von ehemals 26 Betrieben im Jahre 1914 noch 3 und in den Dörfern Marktleuthen und Schwarzenbach gab es nur noch je einen Gerbereibetrieb. Die Gerbereibetriebe in der 
Stadt Hof hatten sich zu Gerbfabriken mittleren Umfangs entwickelt. In den Dörfern war alles beim Alten geblieben. Dort betrieb man noch die Grubengerbung im Nebenerwerb. Die Beschreibung von Eisele (1918) deckt sich mit der reichsweiten Betrachtung des Niedergangs der handwerksmäßigen Gerberei von Gebek (1935, S. 40ff.).

Der gesamte Rindenanfall in Kirchenlamitz wurde im Vorverkauf ausgeboten. Das sicherte der Forstverwaltung einen bestimmten Preis und ermöglichte eine vorteilhafte Kalkulation der benötigten Rindenmenge. Die Verwertung fand ausschließlich auf dem Wege der Versteigerung statt. Es wurde hauptsächlich der Anfall aus den Rechtholzhieben zum Verkauf gebracht. Das kann als ein deutliches Zeichen dafür gesehen werden, dass die Einwände der Holzberechtigten von der Forstverwaltung insgeheim geteilt wurden, denn andernfalls hätte man auch andere Hiebe nutzen können. Der Rindenkäufer hatte dann unter Aufsicht des Forstpersonals die Schälung selbst durchzuführen. Die Verkaufseinheit war das Raummaß des geschälten Holzes. Umgerechnet ergibt sich für die Jahre 1800 bis 1850 ein Durchschnittspreis von etwa 23 Kreuzern pro Zentner lufttrockener Rinde. In den 1870er Jahren stiegen die Rindenpreise kurz stark an, obwohl zu dieser Zeit der Rückgang des Gerbereihandwerks schon begonnen hatte. Eine Verknappung des Rindenangebots hatte diese Entwicklung verursacht (vgl. Eisele 1918).

\section{Die Politik sekundiert: keiner schützt den Schälwald}

In Wirtschaftsbereichen, in denen Produktivitätssteigerungen an natürliche Grenzen stoßen, kommt allgemein schnell die Forderung nach Schutzzöllen auf, um die Absatzchancen heimischer Produktion zu sichern. So auch bei der Lohrindenbewirtschaftung im Niederwaldbetrieb. Das preußische Abgeordnetenhaus fasste 1894 einen entsprechenden Beschluss. Der Reichstag zog 1895 nach und die bayerische Kammer der Abgeordneten 1896. Diese Beschlüsse wurden nun vom Bundesrat noch im Oktober 1896 allesamt verworfen (vgl. Endres 1922, S. 827). Der preußische Handelsminister hatte schon vor dem Bundesratsbeschluss gegen die Position des Abgeordnetenhauses Stellung bezogen. Die 26. Versammlung der deutschen Forstmänner hatte 1898 in Breslau den Beschluss gefasst, dass auf alle ausländischen Gerbstoffe „eine wirksame Zollbelastung“ einzuführen sei. Der Deutsche Forstverein verabschiedete auf seiner Wiesbadener Sitzung 1900 gegen vielseitigen Widerspruch den Beschluss, unter anderem auf Quebrachoholz einen Zoll von 10 Mark pro Doppelzentner zu erheben (vgl. Endres 1905, S. 731). Die Forderungen der Lohrindenproduzenten wurden also über viele Jahre wiederholt. Und doch konnten sie sich nie durchsetzen.

Diesen Zollkampf, den die deutsche Forstwirtschaft gegen die ausländischen Gerbmittel seit Jahrzehnten zu führen bestrebt sei, hätte sie „,bis jetzt immer verloren“" schrieb Endres im Jahre 1922 (S. 823). Denn dem Interesse der Forstwirtschaft stand dasjenige der Lederindustrie entgegen. Diese war zu jener Zeit recht 
bedeutend in Deutschland. Die Lederindustrie beschäftigte 300.000 Arbeiter und trug zu einer positiven Außenhandelsbilanz nicht unerheblich bei (vgl. Endres 1905, S. 824; Eisele 1918, S. 208; Endres 1922, S. 824).

Die Lederindustrie wies auf die Bedeutung ausländischer Gerbmittel für die heimische Lederproduktion hin, ohne die sie nicht konkurrenzfähig sei und forderte deswegen die völlige Zollfreiheit für alle Gerbstoffimporte. Diese Ansicht konnte sich durchsetzen. Ein allgemeiner Zolltarif auf Gerbstoffimporte wurde nicht erhoben (vgl. Endres 1905, S. 731)3. „So betrübend die unabänderliche Tatsache für die Schälwaldbesitzer auch ist: die gedeihliche Entwicklung der mit hunderten von Millionen Mark arbeitenden Lederindustrie ist volkswirtschaftlich wichtiger als die höhere oder geringere Rentabilität von 446000 ha Schälwaldungen“ (Endres 1905, S. 733ff).

Ein Landnutzungswandel: Die Schälwaldwirtschaft wurde großflächig aufgegeben

Die deswegen nahezu ungehinderte Einfuhr großer Mengen billiger ausländischer Gerbstoffe ließ den Preis für heimische Gerbrinde tief fallen. Von 8 bis $10 \mathrm{M}$. je Zentner ab Wald (1870) zu 6 bis 7 M. (1900) und im Frühjahr 1914 auf 2,70 bis 3,40 M. Von diesem Preis waren allerdings noch die Schälkosten abzuziehen, die 1,80 bis 2,50 je Zentner betrugen. Der erzielte Rindenpreis deckte also in der Zeit vor dem Weltkrieg kaum noch die Gewinnungskosten. Die Nutzung von heimischen Eichenwäldern als Schälwald lohnte sich immer weniger. Das hatte forstwirtschaftlich große Folgen. Eine Bewirtschaftungsform, die sich nicht mehr lohnt, wird aufgegeben. Das ließ sich auch beim Eichenschälwald beobachten.

Von 1900 bis 1913 wurde im Deutschen Reich rund 150.000 ha Schälwald aufgegeben. Das entsprach etwa 33 \% der ursprünglichen Fläche. Für Bayern liegen keine eigenen Zahlen vor. Interessant ist jedoch der unterschiedliche Rückgang nach Besitzarten.

\footnotetext{
${ }^{3}$ Auf Eichen- und Fichtengerbrinde wurde zwischen 1879 und 1892 ein Zolltarif von 0,50 M. pro dz. erhoben. 1892 wurde der Zoll aufgehoben. Quebrachoholz war bis 1906 zollfrei, dann wurde der Tarif auf 2 M. je dz. festgesetzt. Das waren Summen, die den Import nicht zu bremsen vermochten.
} 


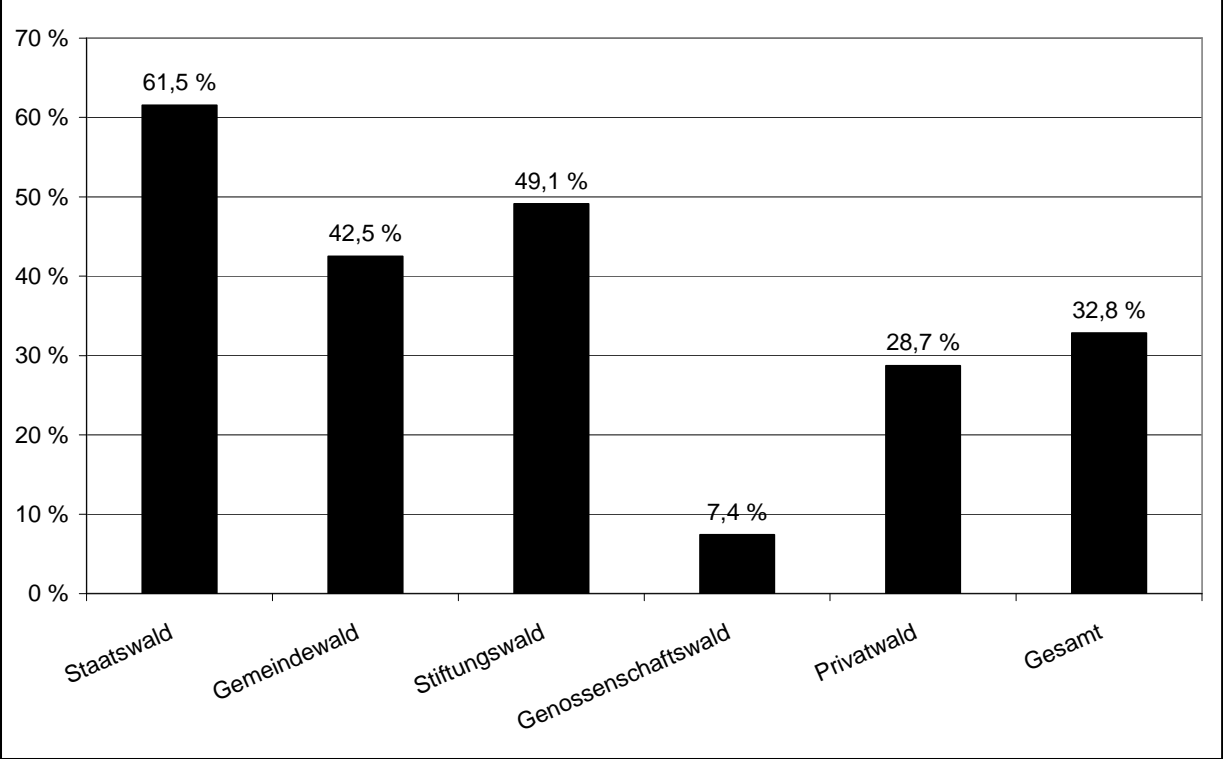

Abb. 3: Prozentualer Rückgang an Eichenschälwaldfläche nach Besitzformen zwischen 1900 und 1913 (Eigene Darstellung, Datengrundlage: Endres 1922, S. 821).

Abbildung 3 zeigt deutlich, dass der Schälwaldrückgang vor allem in staatlichen Forsten stattfand. Hier wurden zwischen 1900 und 1913 61,5\% der Schälwaldfläche umgewandelt. Am geringsten war der prozentuale Verlust im Genossenschafts- und Privatbesitz. Hier konnte sich die Schälwaldwirtschaft also am längsten behaupten. Im letzten Vorkriegsjahr befanden sich nur noch wenige Reste an Eichenschälwald in Staatsbesitz (8821 ha). Die größten Flächen waren im Besitz von Gemeinden oder Privatleuten.

Diese Verteilung des Eichenschälwalds 1913 auf die verschiedenen Besitzformen reflektiert die wirtschaftlichen Präferenzen der Akteure in der Vorkriegszeit. Der Staat als Waldbesitzer konnte mehrjährige Einnahmeausfälle genauso wie die hohen Umwandlungskosten tragen, Gemeinden und Private waren auf stetig fließende Einnahmen angewiesen und konnten sich die teuren Umwandlung meist nicht leisten. Immerhin mussten neben großen Umwandlungskosten auch mehrjährige Einnahmeausfälle getragen werden. Mit größeren Rücklagen und geringerem wirtschaftlichen Druck ließ es sich in den Staatsforsten natürlich langfristiger planen und - aus Sicht der Vorkriegszeit - auch langfristig besser positionieren. Diese Überlegungen führten zu dem Ergebnis, dass ein Jahr vor Kriegsbeginn nur noch $3 \%$ der deutschen Eichenschälwalder in Staatsbesitz waren. 47\% der Schälwälder gehörten Privaten, 31\% Gemeinden. Auch der Anteil der Genossen- 
schaftsforste am Eichenschälwald war mit 18\% inzwischen um ein Vielfaches höher als der der Staatsforste.

Die Zeit der Umwandlung war geprägt von politisch-ideologischen Auseinandersetzungen. Akademisch ausgebildete Förster diffamierten den Niederwaldbetrieb mit seinen typischen Neben- und Zwischennutzungen als „unforstlich“ und „unmodern“. Das Zerrbild einer altmodischen und unrentablen Bauernwirtschaft wurde entworfen. Die Forstwirtschaft begründete ihre ,Herrschaft ${ }^{`}$ über den Wald mit einem starken Fokus auf Nutzholzproduktion im Hochwaldbetrieb. Alle anderen Waldnutzungen wie Waldweide, Streunutzung, Wald-Feldbau und auch althergebrachte Holzberechtigungen sollten abgelöst oder ganz verboten werden. Dass es für Privatwaldbesitzer - in der Mehrheit Bauern und Genossenschaften - andere Nutzungsprioritäten gab, konnten die Förster nicht einsehen. Bauern nutzten ihren Wald als Brennholzacker, sie trieben Vieh ein und nutzten Waldstreu für den Stall. Wenn man nebenher noch die Rinde verkaufen konnte, war das umso besser, aber eben nur eine der vielen möglichen Nutzungen.

Die reine Eichenschälwaldwirtschaft, wie sie in staatlichen Forstämtern betrieben worden, war ab etwa 1870 hingegen kaum mehr rentabel gewesen. Fielen die Rindenkunden weg, war das für die Staatsforstämter nicht so leicht auszugleichen. Die Staatsforstämter hatten in den Vorkriegsjahren selbst mit Brennholz immer weniger Geld verdienen können (vgl. Rubner 1920, S. 92). Lies sich dagegen die Rindennutzung mit anderen Nutzungen kombinieren - das war in Bauernwäldern der Fall - konnten die lokalen Wirtschaftskreisläufe den Landnutzungswandel abbremsen oder auch ganz verhindern; er war deswegen vor allem in den Staatsforstbetrieben zu beobachten.

\section{4: Der Kriegsbeginn unterbrach den Welthandel}

\section{Gerbstoffmangel und staatliche Eingriffe}

Im letzten Vorkriegsjahr weist die amtliche Gerbstoffstatistik nur noch einen Anteil von 18\% aus, der aus Deutschland selbst stammte. Als zu Beginn des Ersten Weltkrieges der deutsche Überseehandel durch die Briten unterbrochen wurde, fand sich die Gerbwirtschaft auf die Nutzung der heimischen Ressourcen zurückgeworfen.

Auf dem deutschen Gerbstoffmarkt kam es zu erheblichen Preissteigerungen. Bei den Versteigerungen in Hessen, in Hirschhorn am Neckar (vgl. AFJZ 1915, S. 153) und in Kaiserslautern (vgl. Silva 15. Januar 1915, S. 44) wurden teilweise bis zu 300\% des letzten Vorkriegspreises erzielt.

Das stellte die Gerber vor große Probleme. Sie sahen ihre Gerbstoffvorräte sinken, dabei winkte durch die stetig steigenden Ledereinkäufe des Militärs ein gutes Geschäft. Die Preissteigerungen bei Gerbrindenversteigerungen verhießen 
für die nahe Zukunft nichts Gutes. Gezieltes Lobbying auf höchster Ebene sollte deswegen die dringend benötigte Rinde beschaffen helfen. Da das Militär innerhalb von wenigen Wochen zum Hauptabnehmer des Leders geworden war, bestand auch auf Seiten des Staates ein großes Interesse an einer billigen Bereitstellung der benötigten Rohstoffe. Gemeinsam mit dem Kriegsministerium gründete der Verband der Gerbereiindustrie darum die Kriegsleder AG. Diese bekam offiziell die Aufgabe zugewiesen, alle im deutschen Machtbereich sich befindlichen Gerbrinden zu übernehmen und an die Gerber weiterzuverkaufen. Gegenüber anderen Kriegsgesellschaften sollte die Kriegsleder AG als Interessensvertretung der Gerbereiindustrie agieren. Die Monopolstellung als Käufer für Gerbstoffe und Tierhäute sowie ihre Bevollmächtigung durch die Staatsmacht versetzte die Kriegsleder AG in die Lage, den deutschen Gerbstoffmarkt zu beherrschen.

\section{Wiederaufnahme der Schälwaldwirtschaft im Krieg}

Durch den Zusammenbruch der Importe zu Beginn des Krieges kam es zu einer kurzzeitigen Wiederbelebung der Schälwaldbewirtschaftung im Deutschen Reich. Das bedeutete, dass noch bestehende Schälwälder wieder und verstärkt ,auf Rinde genutzt wurden. Diese waren allerdings größtenteils in Besitz von Gemeinden und Privaten, sie mussten also mobilisiert werden. Das gelang durch Höchstpreise, die die Waldbesitzer zunächst viel Geld verdienen ließen (vgl. Silva 30. Juni 1916, S. 146) und mithilfe patriotischer Propaganda. Gerbrinde wurde als kriegswichtiger Rohstoff bezeichnet und seine Bereitstellung entsprechend als patriotische Tat verbrämt (vgl. Silva 25. Mai 1917, S. 150, ebenso Stellungnahme des Reichsamts des Innern, IV A 1010, 31. Januar 1915. StA Münster, Forstamt Rumbeck, Nr. 30). Wie die Zahlen der Kriegsleder AG nahelegen, setzte in den Bauernwäldern also ein Landnutzungswandel dergestalt ein, das anstelle der Mehrfachnutzung der letzten Vorkriegsjahre wieder eine verstärkte Nutzung der Lohrinde stattfand (vgl. Silva 1. Juni 1917, S. 161).

Die Steigerung des Rindenanfalls konnte vor allem durch eine Erhöhung des Ertrags pro Hektar Schälwaldfläche erreicht werden. Nach Endres (1905, S. 728) liefert 1 ha Schälwald im Durchschnitt etwa 3,5 Doppelzentner Rinde. Zieht man die Zahlen von 1913 zum Vergleich heran, so kommt man für dieses Jahr auf eine Rindenmenge von nur 0,3 Doppelzentnern pro Hektar Schälwald. Die Lohrindennutzung war in der Vorkriegszeit also kaum mehr ernsthaft betrieben worden.

Das änderte sich mit dem Kriegsbeginn. Die Statistik der Kriegsleder AG (siehe Tab. 2) weist zwar für 1915 nur einen relativ niedrigen Wert aus, es muss aber auch bedacht werden, dass die Gesellschaft ihre Tätigkeit erst zu Beginn des Jahres aufnahm und trotz aller Eile nach nur wenigen Monaten wohl kaum den gesamten deutschen Gerbstoffhandel dominieren konnte. 1916 steigerte sich die Gerbstoffmenge auf das 6,5-fache des Vorkriegswertes. Im folgenden Jahr wurde immerhin noch die fünffache Menge Lohrinde bereitgestellt. Dann sank die Rin- 
denmenge drastisch. Im letzten Kriegsjahr wurden nur noch knapp 20.000 dz. Lohrinde geschält.

Es wurde aber nicht nur die Lohrindenproduktion von noch bestehenden älteren Eichenschälwäldern reaktiviert. Auch junge, erst in den letzten Jahren vor dem Krieg in Hochwälder umgewandelte ehemalige Niederwälder ließen sich ,auf Rinde nutzen. Diese gab es in Staatsbesitz recht häufig (vgl. Endres 1922, S. 821). Hier hatten ja vor dem Krieg die flächenmäßig größten Umwandlungen stattgefunden. Damit machte man zwar die erst kurz zuvor unter hohen Kosten angegangenen Umwandlungen zunichte, aber dem Druck der Gerbereiinteressen konnte sich die Staatsforstverwaltung nicht entziehen. Auch im Staatswald kam es also zu einem kriegsbedingten Landnutzungswandel.

Tab. 2: Lohrindenanfall 1913 bis 1918

\begin{tabular}{l|l|l|l}
\hline Quelle & Jahr & $\begin{array}{l}\text { Gerbstoff aus Eichen- } \\
\text { lohrrindenanfall in dz. }\end{array}$ & $\begin{array}{l}\text { Rindenanfall in dz. pro } \\
\text { ha Schälwald }\end{array}$ \\
\hline Paeßler $(1915,1916)$ & 1913 & 93.400 & 0,3 \\
KLAG (Kriegsleder AG) & 1915 & 114.910 & 0,4 \\
KLAG & 1916 & 628.120 & 2,1 \\
KLAG & 1917 & 484.130 & 1,6 \\
KLAG & 1918 & 19.530 & 0,1 \\
\hline
\end{tabular}

Mit zunehmender Kriegsdauer reduzierten sich die Höchstpreise immer mehr, die Rindennutzung wurde wieder unrentabler und die Propaganda wurde von den Adressaten zunehmend als störend, gar diffamierend empfunden (vgl. Silva 6. April 1917, S. 89, vgl. ebenso Silva 4. Mai 1917, S. 123). Die Rinde war aber auch immer entbehrlicher geworden, da die Gerbereiindustrie zunehmend auf Gerbstoffextrakte aus Hölzern umstieg (vgl. Abb. 4). Es war schon vor dem Krieg bekannt gewesen, dass sich aus Hölzern Gerbstoffe extrahieren ließen, doch waren die Verfahren aufwendig und teuer. 1916 rüstete man einige Destillationsfabriken in besetzen Gebieten an der Ostfront um und so konnten große Mengen Gerbstoff direkt aus den Hölzern gewonnen werden. Die Bereitstellung von solchen Gerbstoffhölzern war organisatorisch einfacher, da sie mit dem normalen Holzeinschlag im Winter erfolgen konnte und nicht wie bei der Rindennutzung, zu der Arbeiter und Transportfahrzeuge im Frühjahr aus anderen Tätigkeiten herausgezogen werden mussten. Der Umstieg von einer Gerbrinden- zu einer Gerbholznutzung bot sich auch wegen der sich abzeichnenden Übernutzung der Schälwälder an. Er ist auf das Jahr 1916 zu datieren, wie die Zahlen der Kriegsleder AG nahelegen (vgl. Abb. 4). Viele der Schälwälder waren in den Jahren vor dem Krieg sehr vernachlässigt worden, und, so kann vermutet werden, bargen deswegen große Effizienzreserven. 1915 und 1916 waren mehrere Jahresschläge 
auf einmal genutzt worden (vgl. Tab. 2). Das war auch vom preußischen Forstminister schon 1914 in einem Erlass so angeordnet worden: Junge Eichenhochwälder, und im kommenden Frühjahr „,mehrere Jahresschläge im Schälwalde“ (Ministerium für Landwirtschaft, Domänen und Forsten, Allgemeine Verfügung Nr. III. 56/1914, 5. November 1914) sollten genutzt werden. Außerdem sei die Ausdehnung von Durchforstungen von Jungbeständen „unbedenklich“ (ebd.). Hinsichtlich der oben aufgeworfenen Frage nach Änderungen der Landnutzung kann also festgestellt werden, dass eine Ausweitung der Rindenschälung im Ersten Weltkrieg erfolgt ist. Bestehende Schälwälder wurden in den ersten beiden Kriegsjahren intensiver ausgebeutet - das legen zumindest die Zahlen nahe - sie waren aber vor dem Krieg weit unter den Möglichkeiten genutzt worden. Es bot sich also an, die untergenutzten Vorkriegsjahre zunächst aufzuholen. Mit der Ausdehnung der Rindennutzung auch auf Durchforstungen setzte ein kurzfristiger Landnutzungswandel auch in Beständen ein, die eigentlich nicht für eine Rindennutzung vorgesehen waren.

\section{Von der Gerbrinden- zur Gerbholznutzung}

Mit der Nutzbarmachung von Destillationsfabriken zur Gerbstoffproduktion ab 1916 ließen sich große Gerbstoffmengen auch aus Hölzern gewinnen. Diese Bereitstellungsform war im Kontext der Kriegswirtschaft einfacher zu gestalten und setzte sich deswegen ab 1916 sehr schnell durch (vgl. Abb. 4). 1917 dominierten bereits die Gerbhölzer den Gerbstoffmarkt, auf dem sie vorher praktisch keine Rolle gespielt hatten. Importierte Extrakte aus verbündeten oder neutralen Staaten spielten dagegen während der gesamten Kriegsdauer nur eine untergeordnete Rolle. Rinden büßten ihre in den ersten beiden Kriegsjahren gewonnene Bedeutung ab 1916 wieder ein. 1918 trugen sie nur noch marginal zur Gerbstoffversorgung bei (vgl. Abb. 4).

Großflächige Umwandlungen von Nieder- zu Hochwäldern oder umgekehrt haben während des Krieges nicht stattgefunden (vgl. Zusammenstellung der wichtigsten Wirtschaftergebnisse in den Staatsforsten des Herzogtums Anhalt für das Wirtschaftsjahr 1916, 1919). Das lag an dem damit verbundenen hohen Arbeitsund Zeitaufwand. Mit den stark gesunkenen Arbeiterzahlen ließen sich größere Kulturarbeiten kaum bewerkstelligen. ${ }^{4}$ Bei noch bestehenden Niederwäldern nutzte man ihre Lohrinde und das Brennholz. Eine Umwandlung konnte man nur für die Nachkriegszeit ins Auge fassen. Ältere Hochwaldbestände wandelte man nicht in Niederwälder um. Das hatte man zwar in den 1850er Jahren so gemacht, aber allen Beobachtern war klar, dass die plötzliche Rentabilität der Rindennutzung auf

\footnotetext{
${ }^{4}$ Für einzelne deutsche Staaten lassen sich die Arbeiterzahlen noch nachvollziehen: Im Herzogtum Braunschweig sank die Zahl der Waldarbeiter zwischen 1914 und 1916 um über 50\%, in den anhaltinischen Staatswäldern um etwa 30\%. Siehe: Statistisches Landesamt (Hg.) (1874 - 1930): Beiträge zur Statistik des Landes Braunschweig, Braunschweig und Anhaltinische Staatsforstverwaltung (Hg.) (1889 - 1923): Die Zusammenstellung der wichtigsten Wirtschafts-Ergebnisse in den Staatsforsten Anhalts, Dessau.
} 
den besonderen Verhältnissen des Krieges beruhte. „Der exotische Gerbstoff ist das Rennpferd und die heimische Rinde ist der Ackergaul, der nur durch eine gute Aufschirrung einigermaßen mitmachen kann“" (Silva 25. Mai 1917, S. 150), schrieb ein Vertreter der Gerbereiindustrie. Die absolute Schälwaldfläche wird sich deswegen kaum verändert haben, auch wenn das heute statistisch nicht mehr zu fassen ist.

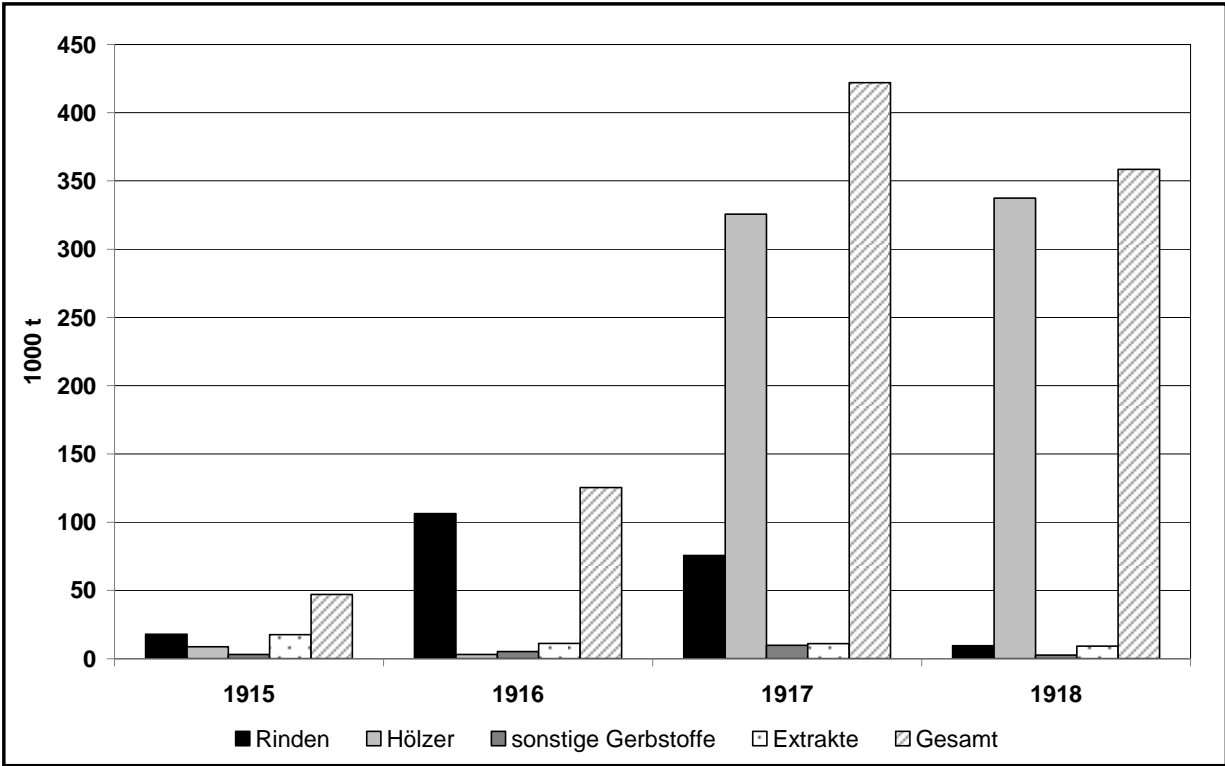

Abb. 4: Menge der einzelnen Gerbrohstoffe (Eigene Darstellung, Datengrundlage: Tabellen der Kriegsleder AG, BA Berlin R 8736).

\section{Nachkriegszeit}

Nach 1919 setzte der Import von Gerbstoffen aus Südamerika rasch wieder ein. Die Aufgabe der meisten Eichenschälwälder und ihre Umwandlung in Hochwälder in der Nachkriegszeit ist als ein Landnutzungswandel zu verstehen, der sich wieder auf die gleichen Bedingungen stützte wie vor dem Krieg: freier Handel und preisgünstiger Transport. Als diese beiden Bedingungen ein weiteres Mal nicht erfüllt werden konnten, kehrte man ebenso rasch wieder zur Rindennutzung zurück wie im Ersten Weltkrieg: Zurzeit der Wirtschaftskrise der 1930er Jahre und der Autarkiepolitik im Dritten Reich kam es noch einmal zu einem Wiederaufleben der Lohrindenproduktion in Deutschland. Bis etwa 1951 lassen sich Rindennutzungen in Deutschland nachweisen. Ab Mitte der 1960er Jahre gehörte das Wissen über die Eichenschälwälder nicht mehr zum Curriculum des forstwissenschaftlichen Studiums in Deutschland. 
Die Niederwaldbewirtschaftung insgesamt erfährt momentan eine Wiederbelebung im Zuge der Energieerzeugung aus Biomasse. Einzelne Niederwälder bringen es auf einen Biomassezuwachs von 10,5 Festmetern pro Hektar (vgl. Ostermann 2002, S. 157). Auch ihr Wert als Biodiversitätsreservoir findet breite Anerkennung (vgl. ebd.). Demgegenüber sind die damals angelegten Hochwaldungen heute als ,Problemzonen“ der Forstwirtschaft bekannt. Minderwertige Samen und allgemein die Anlage von Monokulturen führten zu anfälligen und pflegeintensiven Wäldern.

\section{Fazit}

Im vorliegenden Aufsatz konnte ein Faktor des Landnutzungswandels bestimmt werden, der in der bisherigen Forschung nicht den Stellenwert hat, der ihm eigentlich zukommen müsste: der Krieg. Im Zentrum des Interesses stand die Entwicklung der Gerbrindenproduktion in deutschen Forsten. Zur Erörterung der Leitfrage standen zeitgenössische forstwissenschaftliche Fachzeitschriften und Archivmaterial der Kriegsleder AG zur Verfügung. Auch die Akten eines Forstamtes konnten herangezogen werden. Diese Quellen wurden mithilfe der in der Geschichtswissenschaft üblichen Hermeneutik ausgewertet. Als Ergebnis kann Folgendes festgehalten werden:

In der Mitte des 19. Jahrhunderts war die Gerbereiindustrie im Gebiet des späteren Deutschen Reiches sehr bedeutend. Kleine Handwerksbetriebe verarbeiteten lokale Produkte und es dominierten lokale Märkte. Lediglich einzelne Großbetriebe fertigten für regionale Zentren. Von 1880 an vereinfachten und beschleunigten technische Weiterentwicklungen wie die Fassgerbung den Gerbprozess, erforderten aber ein Umstellen auf Gerbstoffextrakte. Diese ließen sich günstig aus Südamerika importieren und machten die heimische Gerbstoffproduktion in Eichenniederwäldern zunehmend unrentabel. Die Umstellung ließ sich aber in Kleinbetrieben wegen der hohen Investitionskosten nicht durchführen. Die Kleinbetriebe wurden deswegen nach und nach verdrängt.

Diese Entwicklung auf der Abnehmerseite hatte große Auswirkungen auf der Seite der angestammten heimischen Gerbstoffproduzenten: Den Eichenschälwaldbesitzern brachen die Rindenkunden weg. Unter bestimmten Umständen ließen sich Ausweichnutzungen finden, besonders wenn es sich um in der Landwirtschaft tätige Besitzer gehandelt hat. Staatsförstereien verfügten über keine Alternativnutzungen. Sie hatten sich schon seit längerem auf eine Stammholzproduktion fokussiert und wandelten deshalb ihre Niederwälder in Hochwälder um, wenn die entsprechenden finanziellen Ressourcen bereitstanden. 1913 gab es deswegen kaum noch Niederwälder in Staatsbesitz. Hier hatte vor dem Ersten Weltkrieg ein starker Landnutzungswandel eingesetzt. Die Kleinbauern und Genossenschaften dagegen besaßen noch größere Teile ihres ursprünglichen Niederwaldbe- 
standes. Sie hatten ihre Wälder nicht in Hochwälder umwandeln können. Das war schlicht zu teuer gewesen.

Als den Landnutzungswandel hemmende Faktoren können am vorliegenden Beispiel also eine bäuerlich geprägte Besitzstruktur, die Möglichkeit einer flexiblen Mehrfachnutzung der Niederwälder und eine gewisse verkehrstechnische Abgeschiedenheit genannt werden. Auf die untersuchte Region am Fichtelgebirge trafen diese Bedingungen zu.

Mit dem Beginn des Ersten Weltkriegs wurde Deutschland vom internationalen Gerbstoffhandel mit Südamerika vollständig abgeschnitten. Als Folge besann man sich zwangsläufig auf lokale Wirtschaftskreisläufe zurück, die in den Jahren vor dem Krieg stark unter Druck geraten waren und sich nur unter den genannten Umständen hatten halten können.

Die noch bestehenden Eichenschälwälder wurden in den ersten beiden Kriegsjahren stark übernutzt, frische Umwandlungsbestände in Staatsbesitz ebenfalls. In den letzten beiden Kriegsjahren verhinderten allerdings der immer belastendere Arbeitskräfte- und Transportfahrzeugmangel eine weitere großflächige Rindennutzung. Es kann heute außerdem nicht mehr festgestellt werden, ob die Schälwälder nach den starken Übernutzungen der ersten beiden Kriegsjahre überhaupt noch eine weitere Nutzung zuließen. Technische Weiterentwicklungen erlaubten darüber hinaus ab 1916 die Umstellung auf Gerbhölzer, die zu Extrakten weiterverarbeitet wurden. Die Hölzer wurden im Winter mit dem normalen Holz geschlagen, eine aufwendige Freistellung von Arbeitern und Geräten wie bei der Rindennutzung im Frühjahr erübrigte sich hier also.

Das Reaktivieren der Lohrindenproduktion in deutschen Niederwäldern von 1915 bis 1917 ist ein Wandel der Landnutzung, der sich nur mithilfe der besonderen Bedingungen des Krieges erklären lässt. Internationale Verkehrswege, Handelsbeziehungen und Wirtschaftskreisläufe sind instabil. In einem Krieg können sie leicht unterbrochen oder umgelenkt werden. Das Zurückfallen auf lokale Ressourcen ist die Folge.

Eichenlohwälder können somit als Prototypen für den Wandel weiterer Landnutzungsformen angesehen werden. Offensichtlich verdient der Krieg als Faktor des Waldnutzungswandels eine stärkere Beachtung, als ihm in der Forschung bisher zukam. Heutige Reaktivierungsbemühungen der Niederwaldwirtschaft in Form von Kurzumtriebsplantagen stehen in Zusammenhang mit nachwachsenden Rohstoffen als Energieträger. Sie können allerdings an die im Ersten Weltkrieg gemachten Erfahrungen anknüpfen. Eine historische Aufarbeitung vom Wandel verschiedener Waldnutzungen kann so auch für aktuelle Fragestellungen von groBem Gewinn sein. 


\section{Literatur und Quellen}

Allgemeine Forst- und Jagdzeitschrift (AFJZ), Jahrgänge 1914-1921.

Bauer, J., Zormaier, F., Borchert, H. u. Burger, F. (2006): Energieholzmarkt Bayern. Analyse der Holzpotentiale und der Nachfragestruktur. Berichte der bayerischen Landesanstalt für Wald und Forstwirtschaft. Freising.

Bub, G. F. (2003): Waldnutzung und Waldzustand in der mittelrheinischen Grafschaft Wied vom 17. bis 20. Jahrhundert. Landschaftswandel unter gegensätzlichen Ansprüchen. Bonn.

Corvol, A. u. Amat, J.-P. (1994): Forêt et guerre. Paris.

Denecke, D. (2005): Entwicklung in der deutschen Landeskunde und Kulturlandschaftsforschung. In: Fehn, K. u. Simms, A. (Hrsg.): Wege der Historischen Geographie und Kulturlandschaftsforschung. Ausgewählte Beiträge. Zum 70. Geburtstag von D. Denecke. Franz Steiner Verlag. Wiesbaden. S. 11-35.

Deutscher Forstwirtschaftsrat Berlin (1917): Bericht über die Tagung des Deutschen Forstwirtschaftsrates zu Berlin 1916. Berlin.

Eisele [ohne Vorname] (1918): Fichtenlohrindennutzung, in: Forstwissenschaftliches Centralblatt Juni 1918, S. 201-209.

Endres, M. (1905): Handbuch der Forstpolitik. Mit besonderer Berücksichtigung der Gesetzgebung und Statistik. Berlin.

Endres, M. (1922): Handbuch der Forstpolitik. Mit besonderer Berücksichtigung der Gesetzgebung und Statistik. 2. Auflage, Berlin.

Forstamt Rumbeck (o.J.): Landesarchiv Münster, Bestand Forstamt Rumbeck Nr. 30. [ohne Abk.]

Gebek, O. (1935): Der Niedergang der handwerksmäßigen Gerberei. Görlitz.

Geldmacher, K. (2006): Landschaftsentwicklung und Landnutzungswandel im Pazifischen Nordwesten der USA seit 1850. Potsdam.

Konold, W. (1996): Von der Dynamik einer Kulturlandschaft. Das Beispiel Allgäu. In: Konold, W. (Hrsg.): Naturlandschaft Kulturlandschaft. Die Veränderung der Landschaften nach der Nutzbarmachung durch den Menschen. Ecomed. Landsberg. S. 121-136.

Kriegsleder AG (KLAG): Bundesarchiv Berlin. Bestand R 8736.

Kümmerle, T. (2007): Post-socialist land use change in the Carpathians. Berlin. URL: http://edoc.hu-berlin.de/dissertationen/kuemmerle-tobias-2007-0926/HTML/.

Lauffmann, R. (1915): Bericht über die Fortschritte in der Gerbereichemie in den Jahren 1913 - 1915. In: Zeitschrift für Kolloidchemie 1915, S. 36-46. 
Ostermann, R. (2002): Die Niederwälder am Fuß der Ostvogesen (Elsass/Frankreich). Eine kulturgeographische und vegetationskundliche Analyse. Freiburg/Br.

Paeßler, J. (1915): Vorschläge zur Erhöhung unserer Rindenerzeugung. Berlin.

Paeßler, J. (1916): Die Eichenrinde (Sonderabdruck aus der Ledertechnischen Rundschau). Berlin.

Paeßler, J. (1917): Die Bedeutung der Rinde und des Holzes der Edelkastanie für die Lederindustrie. In: Forstwissenschaftliches Centralblatt Oktober 1917, S. 394-411.

Palang, H. (2002): European rural landscapes. Persistence and change in a globalising environment. Dordrecht.

Pott, R. (1985): Vegetationsgeschichte und pflanzensoziologische Untersuchungen zur Niederwaldwirtschaft in Westfalen. Münster.

Rau, M. F. (2005): Land Use Change and Natural Araucaria Forest Degradation Northeastern Misiones - Argentina, Freiburg, URL: http://www.freidok.unifreiburg.de/volltexte/2327/.

Rubner, K. (1920): Die Bewegung der Holzpreise in Deutschland vom Beginn des Weltholzhandels bis zum Weltkrieg. Neudamm.

Silva Forstliche Wochenschrift, Jahrgänge 1914-1918.

Thormann, D. (2002): Untersuchungen zum Landnutzungswandel und Auswirkungen auf ausgewählte Landschaftsfunktionen dargestellt am Beispiel der Untersuchungsgebiete Querfurt und Taucha. Halle.

URL: http://www.ufz.de/data/haase_thormannDA1731.pdf.

http1: http://www.leder-lexikon.de/gerben.php <04.06.2009> 
Der Wald als Psychotop im Spannungsfeld multifunktionaler Landnutzung - das Fallbeispiel Ostalb

Christoph Riegert, Ines Fleer 


\begin{abstract}
The meeting of various claims to the ecosystem forest like these of forestry (profit aim), of the use for recovery (recreation aim), and of nature preservation (protection aim) cannot be carried out without conflicts. To ensure a juxtaposition of the described multifunctions which is for the most part harmonic and free of conflicts, we carried out an analysis of the existent situation.

Empiric surveys and counts of forest visitors as well as standardized interviews with experts and an analysis of license plates were used to pinpoint problems between the named functions.

Like this the different factors of the recreation aim and the profit aim were determined to cause and lead to disturbances within the recreation aim but although between the recreation aim and the profit aim.

An important field of conflict showed in the shape of a deficiency of information and a problem of communication between forestry and the use for recovery as well as in the known problem between the so called slow and fast kinds of recreation.
\end{abstract}

\title{
Multifunktionale Waldnutzung
}

Das Aufeinandertreffen verschiedener Ansprüche an das Ökosystem Wald, wie die der Forstwirtschaft (Nutzfunktion), der Erholungsnutzung (Sozialfunktion) und des Naturschutzes (Schutzfunktion), führt vielfach zu Konflikten zwischen den einzelnen Parteien, die im Sinne nachhaltig multifunktionaler Waldnutzung per gesetzlichem Auftrag gleichberechtigt nebeneinander stehen.

Abbildung 1 verdeutlicht die durch geltendes Waldrecht gesetzlich garantiert gleichberechtigt nebeneinander stehenden Säulen einer integrativ multifunktionalen Waldnutzung. Walderholung als Facette der Sozialfunktion des Waldes steht anderen Ansprüchen wie der Nutz- und Schutzfunktion innerhalb des Funktionsdreiecks gegenüber. Eine nachhaltige multifunktionale Waldwirtschaft muss folglich neben potentiellen Konflikten innerhalb der Sozialfunktion gegenläufige Aspekte zwischen den Säulen einschließlich ihrer Wechselwirkungen identifizieren und Lösungsansätze entwickeln. 


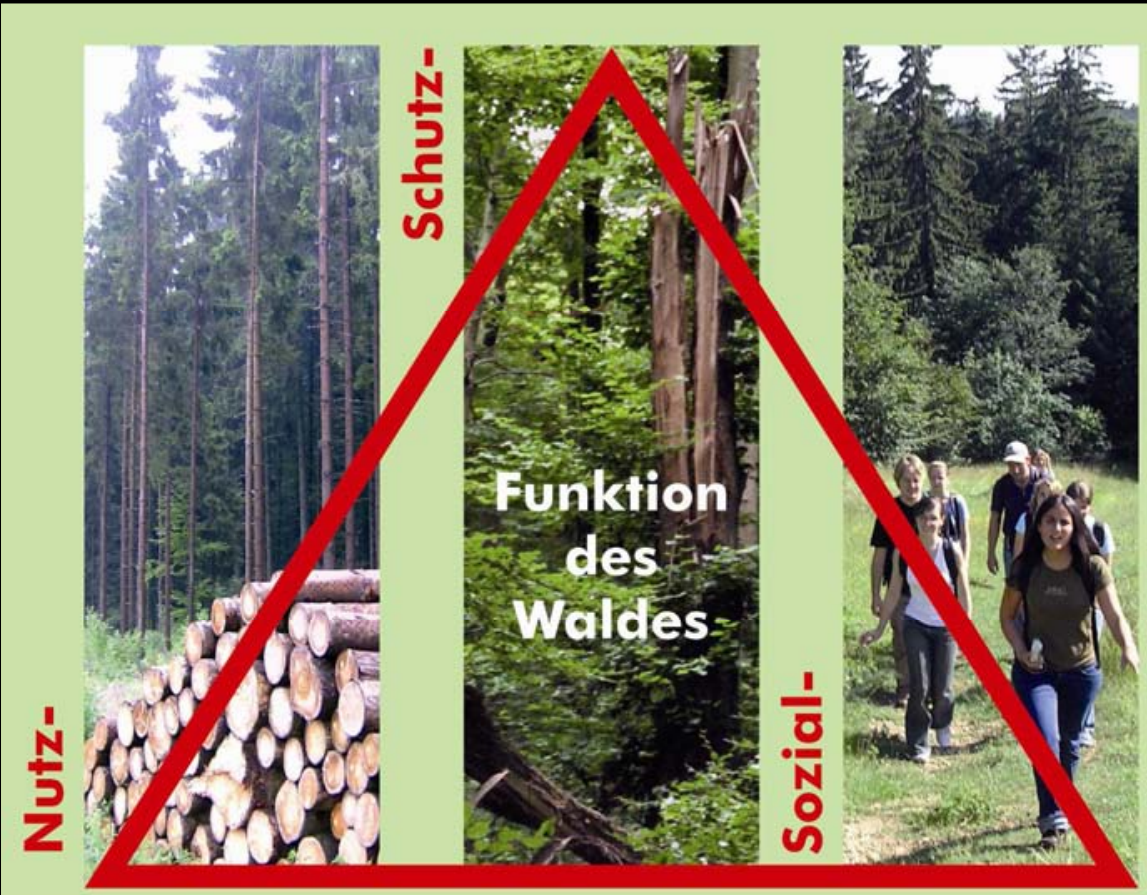

Abb. 1: Ansprüche an das Ökosystem Wald

Unter dem Überbegriff der Sozialfunktion betrachten Erholungssuchende den Wald vor allem als Freizeitraum und Ort der Ruhe und erleben ihn als für sie bereitstehendes Psychotop. Erwartungen gehen hier vor allem in Richtung Naturerlebnis und Erholungskulisse. Als weitestgehend konsum- und werbefreier Raum stellt der Wald ein ideales Umfeld zum „Abschalten vom Alltag“ und zum Regenerieren dar. Ein Psychotop ist demnach ein Rückzugsgebiet für den Menschen und „Wie ein Biotop - wörtlich übersetzt - ein Ort des Lebens, ein Lebensraum ist, so ist ein Psychotop ein Ort der Psyche, der menschlichen ,Seele’ [...] “ (Stölb 2005, S. 60). Stölb fährt fort: „,...] Doch hat es sich eingebürgert, als Biotope nur solche Lebensräume zu bezeichnen, die besonders interessant sind. Wenn wir diese Einschränkung für Psychotope übernehmen, dann gehört der Wald ohne Frage dazu: wird er doch von vielen Leuten nicht aus materiellen Beweggründen aufgesucht, sondern zum Entspannen, Erleben und Wohlfühlen“ (Stölb 2005, S. 61).

Vielfach werden zu Erholungszwecken gezielt naturschutzfachlich besonders wertvolle Landschaftsbereiche aufgesucht, da sie Erholungssuchenden besonders attraktiv und erlebenswert erscheinen (vgl. Riegert u. Kempa 2008). In diesen Fällen treffen Aspekte der Sozialfunktion des Waldes mit denen der Schutzfunkti- 
on aufeinander. Jede ausgeübte Erholungsform formuliert dabei ihre eigenen Ansprüche an Aspekte wie Wegezustand, Relief oder Erreichbarkeit.

Als zweite Säule einer multifunktionalen Waldnutzung stellt die Schutzfunktion die Natur und ökosystemare Zusammenhänge in den Vordergrund. Der Wald soll als naturnahes Ökosystem den Erhalt der Biodiversität, den Arten- und Lebensraumschutz gewährleisten. Darüber hinaus bestehen Ansprüche an anthropozentrisch begründete Waldwirkungen wie Klima-, Wasser-, Boden- und Sichtschutzeffekte. Die Nutzung der natürlichen Ressourcen Wald und Holz ist dabei unter Beachtung naturschutzfachlicher Standards möglich.

Für die Bedienung der Nutzfunktion stellt der Wald ein Rohstofflager dar, welches idealerweise so gepflegt wird, dass eine nachhaltig hochwertige Ertragsmenge an Holz erschlossen werden kann. Hierbei thematisierte Ansprüche lauten unter anderem Ertrag, Verfügbarkeit, Risikominimierung, Arbeitsplätze im Cluster Wald-Holz und effiziente Ressourcennutzung.

Ammer und Pröbstl (1991) fassen zusammen, dass die Ansprüche und Forderungen einzelner Waldnutzungsformen stark variieren und bisweilen gegenläufige Ziele resultieren, die nicht immer in Landnutzungsmodellen harmonisiert werden können.

Die Forstwirtschaft als hauptverantwortlicher Landnutzer des Waldes in Deutschland sieht sich zunehmend neuen Handlungsfeldern (Klimawandel, Förderung nachwachsender Rohstoffe, Reform der Verwaltungsstrukturen, Globalisierung der Märkte) gegenüber. Hierbei scheint die zunehmende Inanspruchnahme von Wald für Funktionen und Umweltwirkungen, die nicht originär der forstlichen (Holz-)Produktion dienen, erheblichen Einfluss auf die strategischen Entwicklungsmöglichkeiten der Waldwirtschaft zu nehmen. Schutzgebiete und Nutzungsauflagen zu Gunsten der Erholungsleistung des Waldes scheinen die Möglichkeiten einer harmonisch multifunktionalen Nutzung der Ressource Wald immer schwieriger zu machen. Es ist dringend geboten dieses Konfliktfeld und seine resultierenden Folgen zu analysieren, um nachhaltige Strategien einer multifunktionalen Waldnutzung zum Wohl aller beteiligten Interessengruppen ableiten zu können.

Ziel der vorliegenden Untersuchung ist die Analyse der Ansprüche der WaldErholungsnutzung, die Identifikation potentieller Konflikte sowie die Ableitung möglicher Lösungsansätze einer nachhaltigen Erholungsnutzung. Denkbare Konfliktfelder werden hierbei sowohl innerhalb der Erholungsfunktion als auch zwischen dieser und den übrigen beiden Funktionssektoren (Nutz- und Schutzfunktion) des Waldes untersucht.

\section{Das Fallbeispiel Ostalb}

Als Untersuchungsgebiet wurde die Ostalb an der östlichen Grenze von BadenWürttemberg ausgewählt. Es handelt sich um einen touristisch kaum erschlosse- 
nen und mit 40\% Bewaldungsanteil sehr waldreichen Landschaftsraum, dessen Siedlungsstruktur als ländlich geprägt zu bezeichnen ist.

Die Erholungsnutzung der Waldgebiete ist vornehmlich durch Nah- und Regionalerholung (Freizeitnutzung durch lokale Anwohner und Tagesausflügler regionaler Herkunft) geprägt. Auf 18\% der Waldfläche wurde im Rahmen der Waldfunktionenkartierung eine besondere Bedeutung der Erholungsfunktion identifiziert. Die Regionalplanung weist darüber hinaus für 43\% des Waldes Vorranggebiete für eine ruhige naturschonende Erholungsnutzung aus.

Durch den hohen Waldanteil der Region spielt die Forst- und Holzwirtschaft eine große Rolle. In der Regionalplanung wird nahezu der gesamten Waldfläche (99\%) besondere Bedeutung für die forstwirtschaftliche Nutzung zugeschrieben.

Für den Naturschutz sind die großen, durch Straßen und Siedlungsflächen wenig zerschnittenen Waldflächen mit ihrer ausgeprägten Habitatkontinuität von enormer Relevanz. Eine Vielzahl unterschiedlicher schutzwürdiger Gebiete nach Naturschutzrecht (Fauna-Flora-Habitat-, Vogelschutz-, Landschaftsschutz und Naturschutzgebiete sowie Naturdenkmale oder gesetzlich geschützte Biotope) überlagern sich auf selber Fläche und betreffen 49\% des Waldes (Daten vgl. Riegert, Kempa u. Bürger-Arndt 2009, S. 72).

\section{Untersuchungsdesign}

Mit 20 ausgewählten Akteuren („Experten“ bzw. „Fachvertretern“ der Schutzund Sozialfunktion des Waldes, in Anlehnung an Meuser und Nagel 1991) wurden standardisierte zweistündige Interviews durchgeführt. Ziel war es, regional erwünschte Waldwirkungen und Anforderungen an die Forstwirtschaft zu identifizieren. Zur Validierung der Experteninterviews und weiterführenden Analyse des Spannungsfeldes multifunktionaler Waldnutzung erfolgte eine Befragung von Waldbesuchern. An verschiedenen Wald-Erholungsschwerpunkten (durch die Waldfunktionenkartierung als Orte mit starkem Erholungsbetrieb ausgewiesen) wurden 404 Personen zum Themenfeld Forstwirtschaft, Walderholung und Naturschutz befragt. Durch die Erfassung waldbezogener Ansprüche und Vorstellung der Befragten konnte auf diese Weise eine Konfliktanalyse gemäß der vorgestellten Zielsetzung umgesetzt werden.

Eine parallel vorgenommene Zählung der vorkommenden Erholungsarten erfasste 1.655 Waldbesucher. Darüber hinaus konnten 595 Fahrzeuge auf repräsentativ ausgewählten Waldparkplätzen im Rahmen einer systematischen Kennzeichenanalyse untersucht werden. Ein besonderer Fokus lag dabei auf der Identifikation der Ansprüche, die verschiedene Erholungsformen an den Wald stellen, und der aus diesen Forderungen entstehenden Konflikte zwischen und innerhalb den Waldnutzungsformen. Darüber hinaus sollten belastbare Daten zur Abschätzung der Bedeutung touristischer und regionaler Erholungsaktivitäten gewonnen werden. 
Als Walderholung verstehen die Autoren alles, was der körperlichen Regeneration dient und im Wald stattfindet. Dabei ist der Ort der Tätigkeit namengebend und nicht die Abhängigkeit der Tätigkeit vom Ort, d.h., auch solche Erholungsarten wie das Spazieren gehen oder Radfahren sind als Walderholung aufzufassen, obwohl sie auch an anderen Orten ausgeübt werden könnten.

Aufgrund der hohen Bedeutung der Nah- und Regionalerholung im Untersuchungsgebiet wurden im Rahmen der Untersuchung Aspekte der Naherholung weiter differenziert, um belastbare qualitative wie quantitative Aussagen ableiten zu können.

Naherholung definieren Ammer und Pröbstl (1991) als Erholungsnutzung in einer Maximalentfernung von $50 \mathrm{~km}$ vom Wohnort, das entspricht in etwa einer Fahrtzeit von einer Autostunde. Für die Naherholung im Untersuchungsgebiet wurde demgegenüber eine weitere Differenzierung vorgenommen: Als Anwohner gelten Personen, deren Wohnort höchstens $5 \mathrm{~km}$ vom Wald entfernt liegt. Als Naherholer werden Erholungssuchenden bezeichnet, die mehr als $5 \mathrm{~km}$ und maximal $30 \mathrm{~km}$ vom Untersuchungsort entfernt wohnen und somit nicht mehr als ca. 30 Minuten Fahrtzeit für ihren Erholungsbesuch in Kauf nehmen. Waldbesucher aus mehr als $30 \mathrm{~km}$ und bis zu $50 \mathrm{~km}$ entfernt liegenden Herkunftsregionen (ca. 60 Minuten Anreisezeit) stellen die Gruppe der relativen Naherholer. Darüber hinaus wurden die Kategorien „> $50 \mathrm{~km}$ bis 100 km“, „> 100 km“ sowie „Erholungssuchende ausländischer Herkunft" unterschieden.

\section{Erholungsnutzung der Ostalb}

Nach Aussage der Fachvertreter erfolgt die Erholungsnutzung der Ostalb vorwiegend lokal bis regional, so dass im Untersuchungsgebiet nur sehr bedingt von einer touristischen Nutzung der Landschaft gesprochen werden kann (vgl. Friedhoff 2006, S. 60). Die Untersuchungsregion ist durch Kurz- und Tageserholung geprägt. Der Anteil der Anwohner und Naherholer im Untersuchungsgebiet beträgt insgesamt $68 \%$. Hinzu kommt ein Anteil relativer Naherholer, die bis zu einer Stunde Anreisezeit investieren. Nur 10\% der befragten Waldbesucher gaben einen Herkunftsort in 50 bis $100 \mathrm{~km}$ Entfernung an. Noch weitere Anreiseentfernungen (über $100 \mathrm{~km}$ ) nannten 9\% der Befragten. Als bedeutsame Formen der Walderholungsnutzung benennen die Fachvertreter Mountainbiken $(80 \%$ der Nennungen), Wandern (70\% der Nennungen), Spazierengehen (55\% der Nennungen) und Nordic Walking (50\% der Nennungen). Erholungsformen wie Radfahren und Reiten nehmen mit 45\% der Nennungen mittlere Häufigkeitswerte ein. Die Auswertung der Waldbesucherzählung und -befragung zeigt hingegen Spazieren gehen und Wandern als wichtigste Erholungsformen im Untersuchungsgebiet. Mountainbiking als auffallende, vielfach konfliktbehaftete und im Untersuchungsgebiet stark „im Aufwind“ befindliche Erholungsart wurde durch die Experten in seiner gegenwärtigen Bedeutung überschätzt. 
Eine wichtige Grundlage für die Einschätzung der Erholungssituation und darauf aufbauender Verbesserungsvorschläge sind die Wünsche und Erwartungen der Erholungssuchenden an den Wald. Die Abbildungen 2, 3, 4, 7 und 8 zeigen die Ergebnisse der Waldbesucherbefragung in Hinblick auf die Anspruchsprofile an einen Erholungswald.

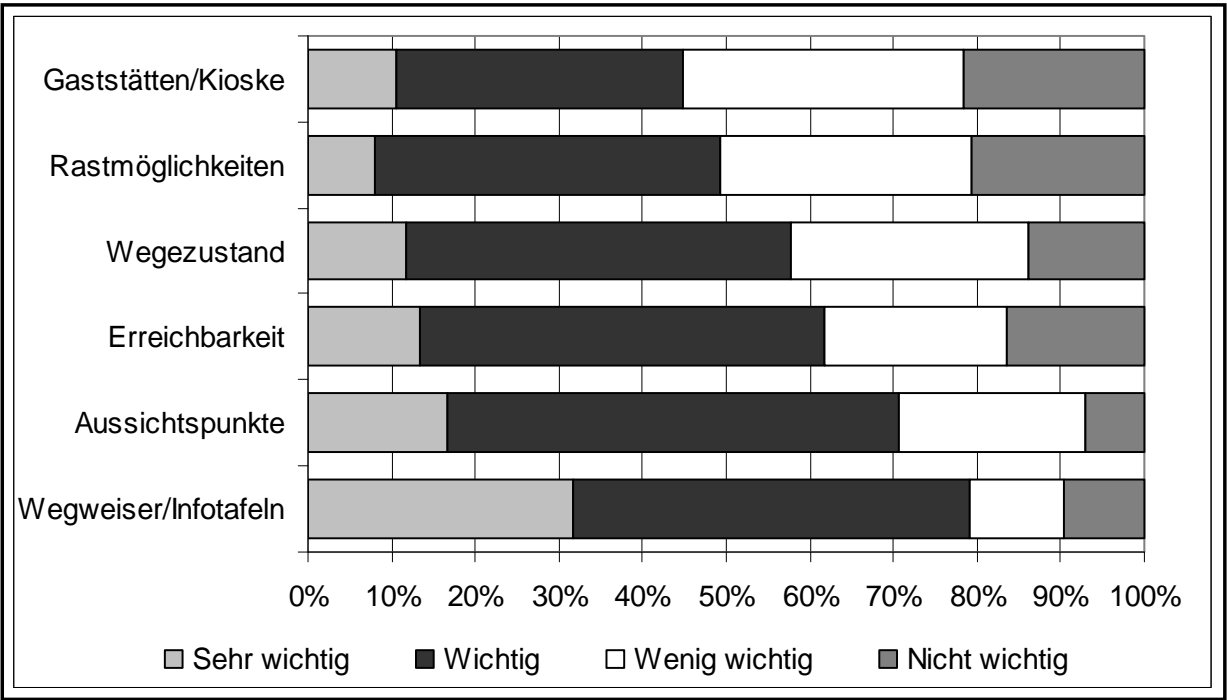

Abb. 2: Bedeutung der Erholungsinfrastruktur für die Walderholung

Hinsichtlich der Erholungsinfrastruktur achten insgesamt 57\% der befragten Waldbesucher verstärkt auf einen guten Wegezustand. Dessen Bedeutung wird durch den Aspekt der Erreichbarkeit des Erholungsraumes weiter betont.

Ein guter Wegezustand (20\% der Nennungen) sowie schlüssige Beschilderungskonzepte (45\% der Nennungen) werden auch nach Expertenmeinung als besonders bedeutsam für die Erholungssituation auf der Ostalb eingeschätzt. Ein Ausbau des existierenden Erschließungsnetzes wird jedoch nur lokal als erforderlich bewertet und der gegenwärtige Stand insgesamt als ausreichend beurteilt. Unter einem „guten“ Wegezustand verstehen hierbei viele der Befragten einen wassergebundenen Wegekörper (20\% der Nennungen), 15\% nennen unbefestigte Waldwege und Fußpfade. In 10\% der Interviews wurden Teerdecken als präferierte Wegeform eingeschätzt. Der überwiegende Teil der Antworten (55\% der Nennungen) konnte keiner speziellen Wegeform zugeordnet werden. 


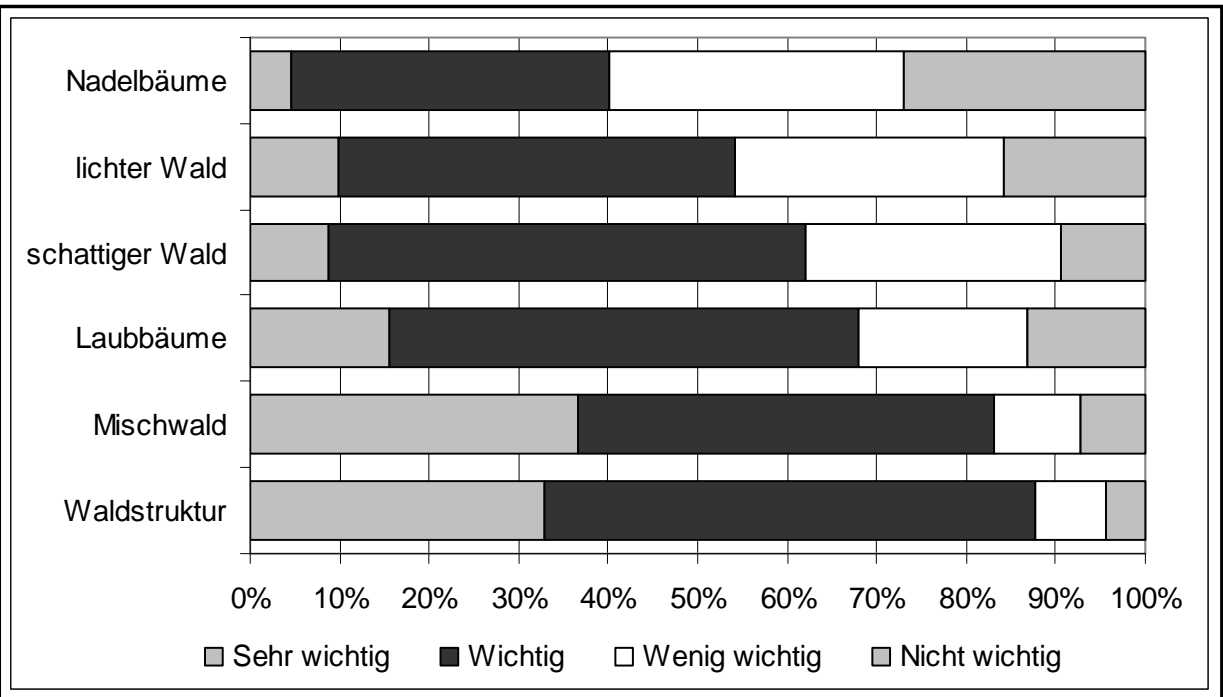

Abb. 3: Bedeutung des Waldbildes für die Erholung

Alle zur Wahl gestellten Eigenschaften des Waldbildes wurden von der Mehrheit der Befragten als überwiegend wichtig für die Erholung angesehen. Deutlich über $50 \%$ favorisieren einen eher schattigen, struktur- und laubholzreichen Mischwald. Die Präsenz von Nadelbäumen wird dagegen nur von 36\% der Befragten als wichtig, und von einem kleinen Teil als sehr wichtig angesehen. Die Mehrheit der Waldbesucher $(60 \%)$ legt darauf keinen besonderen Wert. Diese Ergebnisse decken sich mit den Aussagen der Experten. Nach Auffassung der Fachvertreter gelten insbesondere strukturierte und abwechslungsreiche laubholzdominierte Waldbilder (61\% der Nennungen) und Mischwälder (50\% der Nennungen) als besonders attraktiv für die Erholungsnutzung. Reine Nadelholzbestände erscheinen hingegen unattraktiv (39\% der Nennungen). Einen Baumartenwechsel oder Veränderungen in der Waldstruktur würden nach Einschätzung der Experten bei „sanfter Änderung“ des Waldbildes nicht oder nur kaum durch die Erholungssuchenden wahrgenommen werden. 


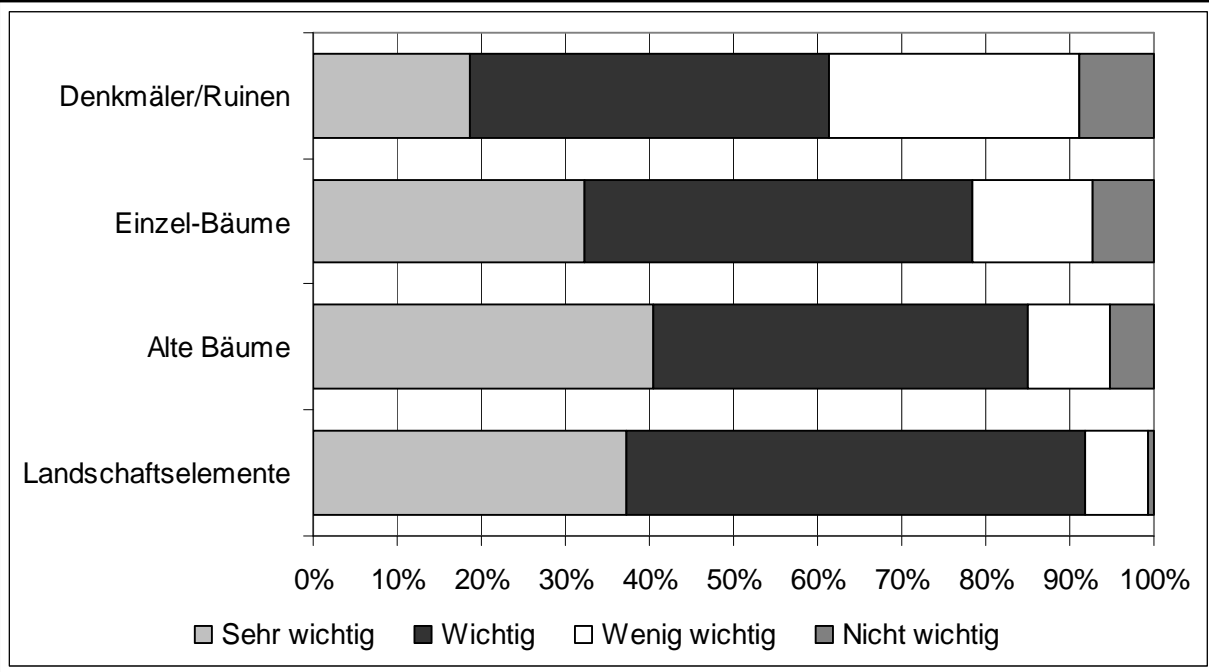

Abb. 4: Bedeutung des Landschaftsbildes für die Erholung

Deutlich vor anthropogen geschaffenen Landschaftsobjekten wie Denkmälern oder Ruinen nehmen natürliche Elemente wie „Baumindividuen“ oder Landschaftselemente (z.B. Felsen) einen besonderen Stellenwert für die Erholung ein. Auch im Zuge der Experteninterviews wurden markante natürliche Landschaftselemente (28\% der Nennungen) neben Wald(innen)rändern (22\% der Nennungen) als wichtiges Element der Erholungskulisse genannt. Erholungssuchende entwickeln bei häufigen Waldbesuchen - wie sie für die lokale und regionale Erholungsnutzung typisch sind - eine emotionale Bindung an einzelne Baumindividuen, Wald- und Landschaftsbilder.

Eine abwechslungsreiche, vielgestaltige Landschaft mit Aussichten und Ausblicken ist nach Meinung von 40\% der befragten Experten ein attraktiver Erholungsfaktor und spielt auf der waldreichen Ostalb eine besondere Rolle. Vor allem die regional typischen Wacholderheiden als markante Landschaftsteile tragen dazu bei, dass Landschaftsbilder als schön und für die Erholung als geeignet empfunden werden (35\% der Nennungen).

Die bewusste Wahrnehmung der Ostalb als eigenständiger „Landschaftstyp“ findet jedoch nur sehr eingeschränkt statt. Erholungssuchende konzentrieren sich vielmehr auf markante Ausflugsziele (Erholungsschwerpunkte) als auf die Landschaft selbst und nehmen das Landschaftsbild OSTALB nur indirekt und erst bei wiederholter Nutzung bewusst wahr. Insbesondere an Konzentrationspunkten der Erholungsnutzung sollte die Landnutzung folglich Rücksicht auf die Erholungsfunktion und die Landschaftsästhetik nehmen. 


\section{Konfliktempfinden der Erholungsnutzer}

Der Großteil der Erholungssuchenden reagiert äußerst sensibel auf Veränderungen in einer vertrauten Umgebung. Vor allem das nach einem forstlichen Eingriff zurückbleibende Waldbild erregt bei vielen Besuchern Anstoß (farbige Markierungen an Bäumen, Auflichtung des Bestandes, Fahr- und Maschinenspuren, großdimensionierte Holzpolter). Nach einer Zeit der Gewöhnung und der natürlichen Weiterentwicklung wird der neue Waldzustand jedoch vielfach als positiv empfunden.

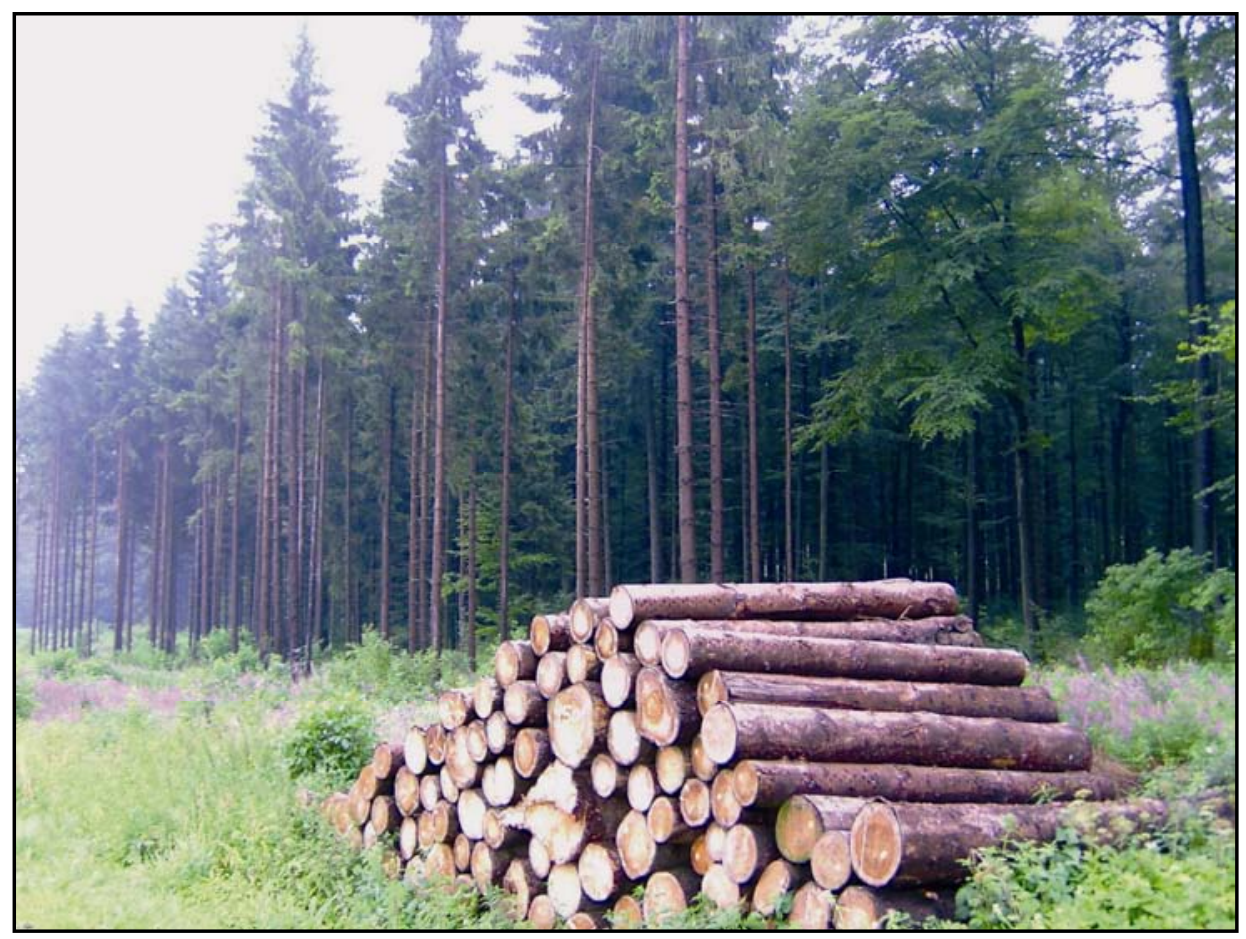

Abb. 5: Forstbetrieblich bedingte Veränderungen im Waldbild (Foto: Riegert)

Abbildung 5 verdeutlicht wie die forstliche Nutzfunktion Einfluss auf das Erholungspotential des Waldes nimmt. Die dargestellte Waldinnenrandgestaltung verbunden mit einer Förderung der Laubbäume bei gleichzeitiger Rücknahme des Fichtenanteils führt zu einem positiven Effekt für den Erholungswert der betroffenen Fläche. Kleindimensionierte Holzpolter, und bodenschonende Holzernte sorgen für ein konfliktfreies Nebeneinander von Nutz- und Erholungsansprüchen auf selber Fläche.

Gefragt nach der Nutzung des Waldes durch die Forstwirtschaft geben $60 \%$ an, dass das Ausmaß der forstlichen Nutzung beibehalten werden könne. Ein 
kleiner Teil (11\%) der Waldbesucher empfindet die Nutzungsintensität der Forstwirtschaft auf der Ostalb als zu stark und gibt an, dass diese eingeschränkt werden sollte. Noch weniger Erholungssuchende (8\%) wären dagegen mit einer Intensivierung der forstwirtschaftlichen Nutzung einverstanden.

Im Hinblick auf die zukünftige Waldbewirtschaftung fordern die regionalen Fachvertreter der Schutz- und Sozialfunktion nachfolgend zusammengestellte Mindeststandards: Naturgemäße Waldwirtschaft und die nachhaltige Sicherung der Wasserschutzfunktion stellen den wichtigsten Anspruch an die Waldwirtschaft auf der Ostalb dar. Deutlich lehnen die Fachvertreter eine Ausweitung des als ökologisch schädlich und auch der Erholungswirkung des Waldes abträglich eingeschätzten Großmaschineneinsatz im Zuge vollmechanisierter Holzernte ab. Abrupte Änderungen im Waldbild sollten bei künftig Waldbauformen vermieden und durch einzelstammweise oder kleinflächige Nutzung realisiert werden. Wegen ihrer besonderen ökologischen Funktion und ihrer positiven Wirkung auf Erholungssuchende sollten insbesondere Waldränder und Offenbereiche im Wald als bedeutende Landschaftselemente erhalten und schroffe Übergänge zu anderen Nutzungsformen vermieden werden.

Darüber hinaus existieren Vorstellungen im Hinblick auf eine zukünftige Baumartenzusammensetzung und Waldstruktur. 20\% der Befragten führen aus, dass gewisse Anteile fremdländischer Baumarten (Douglasie) akzeptabel seien. Gegen den eingeschränkten Anbau fremdländischer Baumarten, sei nichts einzuwenden, solange Maximalanteile (10\%) nicht überschritten würden. Eine weitere Ausdehnung reiner Nadelholzbestände wird abgelehnt. Die zukünftige Waldwirtschaft sollte einen Beitrag zur Förderung heimischer Baumarten liefern. Nach Einschätzung der Experten erkennen Waldbesucher in der Regel keine Baumarten sondern nehmen allenfalls Unterschiede im Waldbild wahr und unterscheiden zwischen Laub-, Nadel- und Mischwald.

Wichtig für die Einschätzung der vorliegenden Konfliktsituation im Wald war vor allem eine Bewertung der Inanspruchnahme des Waldes durch die Forstwirtschaft oder aus naturschutzfachlichem Motiv durch die befragten Erholungssuchenden. Wie erwartet stellt vor allem der Naturschutz keinen Störfaktor für die Waldbesucher dar, er läuft zumeist unbemerkt im Hintergrund ab. Zudem wurde die Bevölkerung in den letzten Jahren stark für Natur- und Umweltschutzaspekte sensibilisiert. Lediglich naturschutzfachlich bedingte Sperrungen (z.B. an Kletterfelsen, die als Brutstätte gefährdeter Arten dienen), führen zu Unmut, da sich betroffene Sportler in ihren Entfaltungsmöglichkeiten eingeschränkt fühlen. Einen derart gesperrten Felsen zeigt Abbildung 6 nebst einem erfolgreichen Beispiel des Arbeitskreises Klettern und Naturschutz (AKN) Oberes Donautal zur gelungenen Kommunikation zwischen Naturschutzvertretern und Erholungsnutzern. 


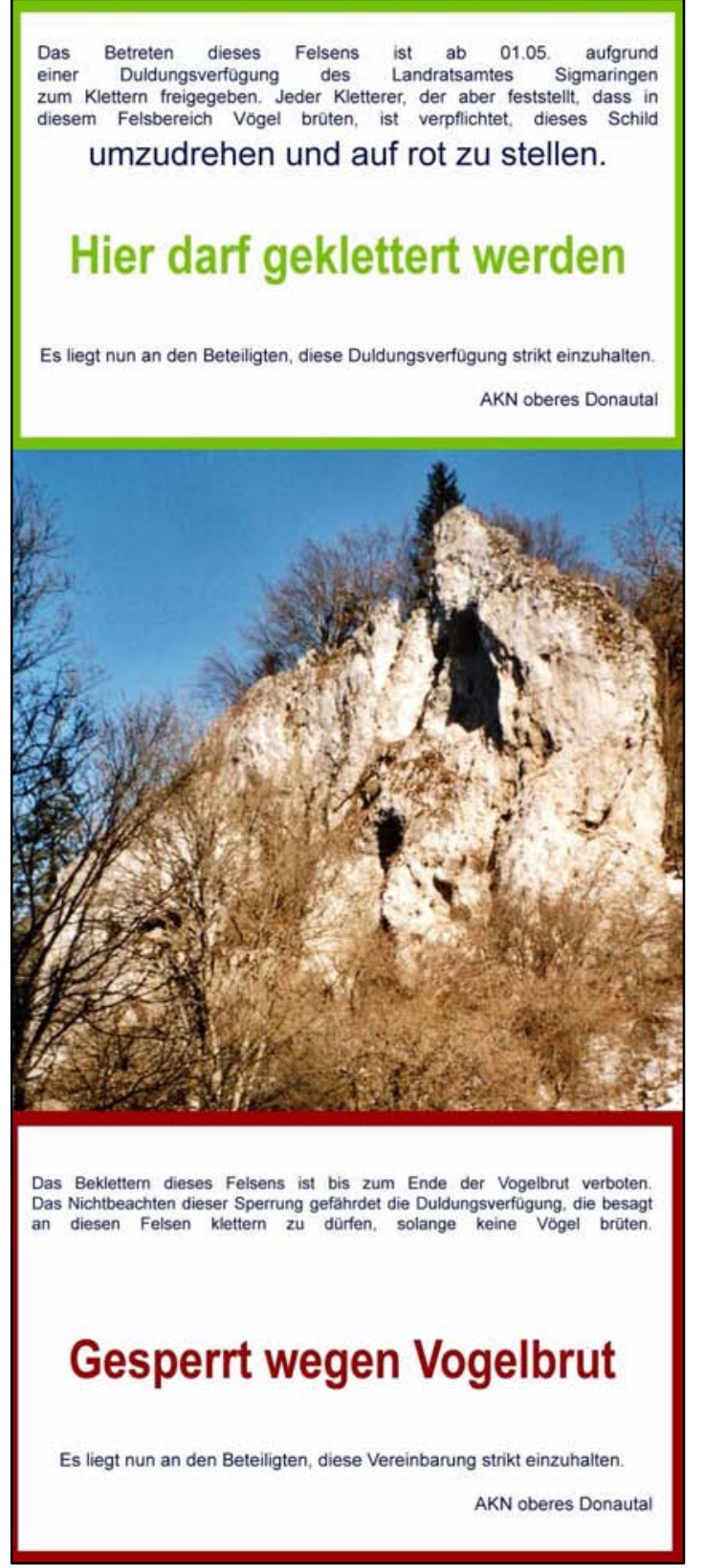

Abb. 6: Der naturschutzfachlich bedingt gesperrte Hirschfelsen sowie ein BeschilderungsBeispiel erfolgreicher Kommunikation zwischen Naturschutzvertretern und Erholungsnutzern (Foto: Riegert) 
Eine Beeinträchtigung ihrer Erholung durch forstwirtschaftliche Arbeiten führt mit 18\% ein geringer Teil der Befragten an. Aufgrund des hohen Waldanteils im Untersuchungsgebiet kann eine weitreichende Akzeptanz forstbetrieblicher Maßnahmen bei der Bevölkerung vorausgesetzt werden. Dennoch empfundene Störungen stehen erwartungsgemäß zumeist in Zusammenhang mit im Zuge von Baumfällarbeiten beschädigten oder schwer passierbaren Wegen, aber auch mit Lärm sowie der grundsätzlich empfundenen Zerstörung des Waldes. Abbildung 7 zeigt die Bedeutung verschiedener durch die Forstwirtschaft hervorgerufener Störungen aus Sicht der Erholungssuchenden.

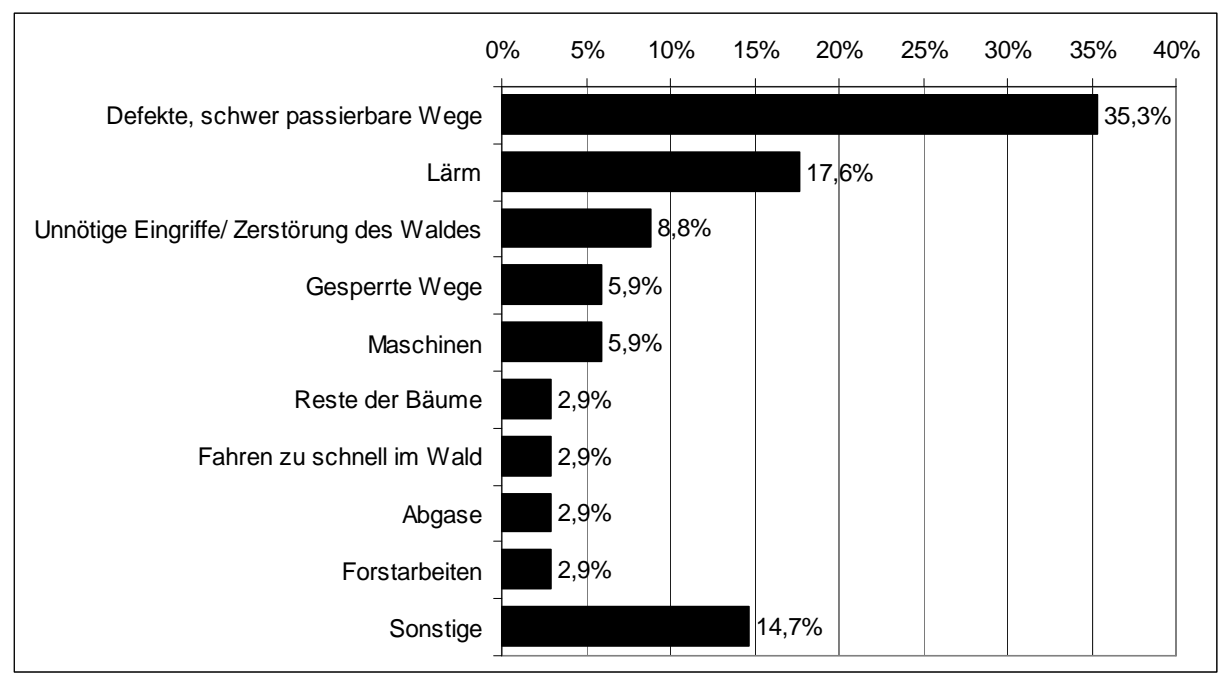

Abb. 7: Durch die Forstwirtschaft hervorgerufene Störungen der Erholungssuchenden

Insbesondere der Aspekt der empfundenen Waldzerstörung durch forstliche Arbeiten wurde auch in anderen Untersuchungen festgestellt (vgl. Mann 2006, S. 95). Stölb (2005, S. 14) begründet dies mit dem negativen Bild der Forstwirtschaft, die „die Reste unserer in die Wälder zurückdrängenden Natur ausbeutet [...]“. So steht Wald vor allem für Natur und Wildnis, an der die Forstwirtschaft Raubbau betreibt. Im Zusammenhang mit Waldsterben und $\mathrm{CO}_{2}$-Ausstoß handelt es sich hier um eine negative Publicity, die in den letzten Jahrzehnten die Medienwelt und damit auch die Medienempfänger stark prägte.

Bezüglich des Störungsempfindens Erholungssuchender durch andere Waldbesucher konnten vor allem Lärm und Rücksichtslosigkeit identifiziert werden, aber auch zu hohe Besucherdichten und Müll wurden durch die Befragten genannt. Neben einer unbestimmten Zuordnung ,es kann jeder sein' wurden als Störungsverursacher vor allem Mountainbiker und Jugendliche genannt, die insbesondere auch für die zuvor genannten Störungen verantwortlich seien. 


\section{Konfliktverständnis der Erholungsnutzer}

Neben der reinen Identifikation der Konfliktfelder ist für eine umfassende Analyse bedeutsam, mit welchem Konfliktverständnis und Wissenshintergrund Erholungssuchende den Wald nutzen und sich ihre Meinung über die Forstwirtschaft bilden (vgl. Fleer 2008, S. 96). Hier können einige starke Wissensdefizite aufgedeckt werden: Beispielsweise benennen 44\% der Befragten das „Bäume fällen“ als Aufgabe der Forstwirtschaft und nehmen die damit zusammenhängenden Aspekte wie Sperrungen oder Forstmaschinen wahr. Die im Zuge geregelter Forstwirtschaft jedoch hiermit im unmittelbaren Zusammenhang stehenden Wiederaufforstungen und Kulturmaßnahmen werden nur von 6\% der Befragten der Forstwirtschaft zugeordnet. Wegeunterhaltung und Forstwegebau verstehen nur 4\% unter forstbetrieblicher Arbeit. Kaum einem Waldbesucher sind Arbeiten wie Wildschadensverhütung bewusst. Es kann folglich ein deutlicher Wahrnehmungsfokus auf den Bereich der Holzernte festgestellt werden. Ein Bewusstsein bezüglich gesamtökologischer, naturschützerischer oder auch administrativer Aufgaben ist nicht auszumachen. Doch gerade diese kaum im Zusammenhang mit der Forstwirtschaft (Nutzfunktion) wahrgenommenen Bereiche gelten den Erholungssuchenden als sehr wichtige Aufgaben im Wald. In einer weiteren Frage waren die Waldbesucher aufgerufen zu beurteilen, welche Bedeutung bestimmte Handlungsfelder als Aufgabenbereich der Forstwirtschaft haben sollten. Dabei zeigte sich, dass etwa 60\% den Umwelt- und Naturschutz als Aufgabenbereich der Forstwirtschaft als sehr wichtig beurteilen. Die Holzproduktion hingegen wird nur von $30 \%$ in derselben Weise bewertet. Die von den Erholungssuchenden primär wahrgenommenen Aspekte der Forstarbeit werden also wesentlich geringer geschätzt als die eben nicht als originäre Aufgaben identifizierten Aspekte wie Waldpflege und -schutz.

Abbildung 8 verdeutlicht dieses Ergebnis. Es ist zu erkennen, dass das Aufgabenfeld Umwelt- und Naturschutz von einer deutlichen Mehrheit der Waldbesucher als wichtiger bzw. sehr wichtiger Aufgabenbereich der Forst angesehen wird. Mit der Holzproduktion, der Freizeitnutzung des Waldes und den Schutz und Pflegeaspekten werden Bereiche aller drei Säulen einer multifunktionalen Landnutzung als bedeutsame Aufgabenbereiche bewertet. Die Jagd hingegen schätzt nur gut die Hälfte der Waldbesucher als wichtiges bzw. sehr wichtiges Wirkungsfeld der Forstwirtschaft. 


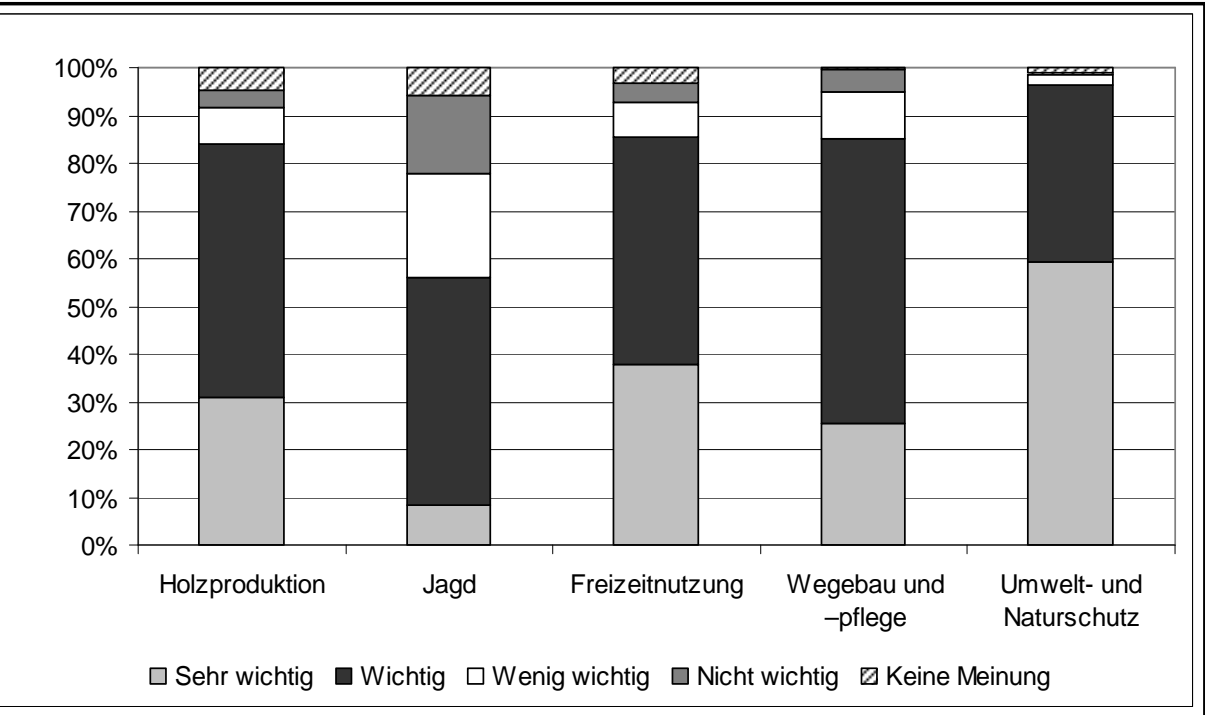

Abb. 8: Beurteilung der Aufgabenbereiche der Forstwirtschaft

\section{Lösungsansätze und Konfliktmanagement}

Aus der Analyse der erfassten und beschriebenen Situation heraus, ergeben sich verschiedenste Ansatzpunkte, um Konflikte um die Ressource Wald zu entschärfen.

Am einfachsten scheint dabei eine Lösung für die Probleme zwischen verschiedenen Erholungsarten zu sein. Durch eine räumliche Trennung „schneller“ und „langsamer" Erholungsformen kann eine Konfliktentschärfung erwartet werden, wobei die Einrichtung von Mountainbiketrails im Untersuchungsgebiet als erfolgreiches Beispiel angeführt werden kann.

In gleicher Weise kann durch räumliche Trennungen, z.B. durch Sichtunterbrechungen oder weitläufig erschlossene Gebiete eine Entschärfung des so genannten „Crowding-Effektes“ erreicht werden, d.h. eine Verteilung der Besuchermengen im Erholungsraum (Mann 2006, S. 102ff).

Der beschriebene Informationsmangel der Waldbesucher zeigt, dass es deutliche Diskrepanzen zwischen der Eigeneinschätzung der forstlichen Landnutzung und der Wahrnehmung durch die Bürger gibt. Gerade hier existiert ein Ansatzpunkt, an dem Aufklärungsarbeit geleistet werden kann und sollte, um das Bild der Forstwirtschaft auch in der Öffentlichkeit positiv zu belegen.

Die Forstwirtschaft erbringt große Leistungen im Bereich der Waldpflege, des Naturschutzes und zu Gunsten der Erholungsnutzung (z.B. Borkenkäferbekämpfung, Biotoppflege, Verkehrssicherung, Offenhalten von Ausblicken). Diese soll- 
ten stärker verdeutlicht werden, um in der Bevölkerung größere Akzeptanz zu erreichen.

Ansprechende interaktive Informationstafeln und in Öffentlichkeitsarbeit geschulte Forstbedienstete könnten im Wald aber auch in nahegelegenen Städten persönlich über die Arbeit informieren und so Aufmerksamkeit und Verständnis für die Forstarbeit erzielen.

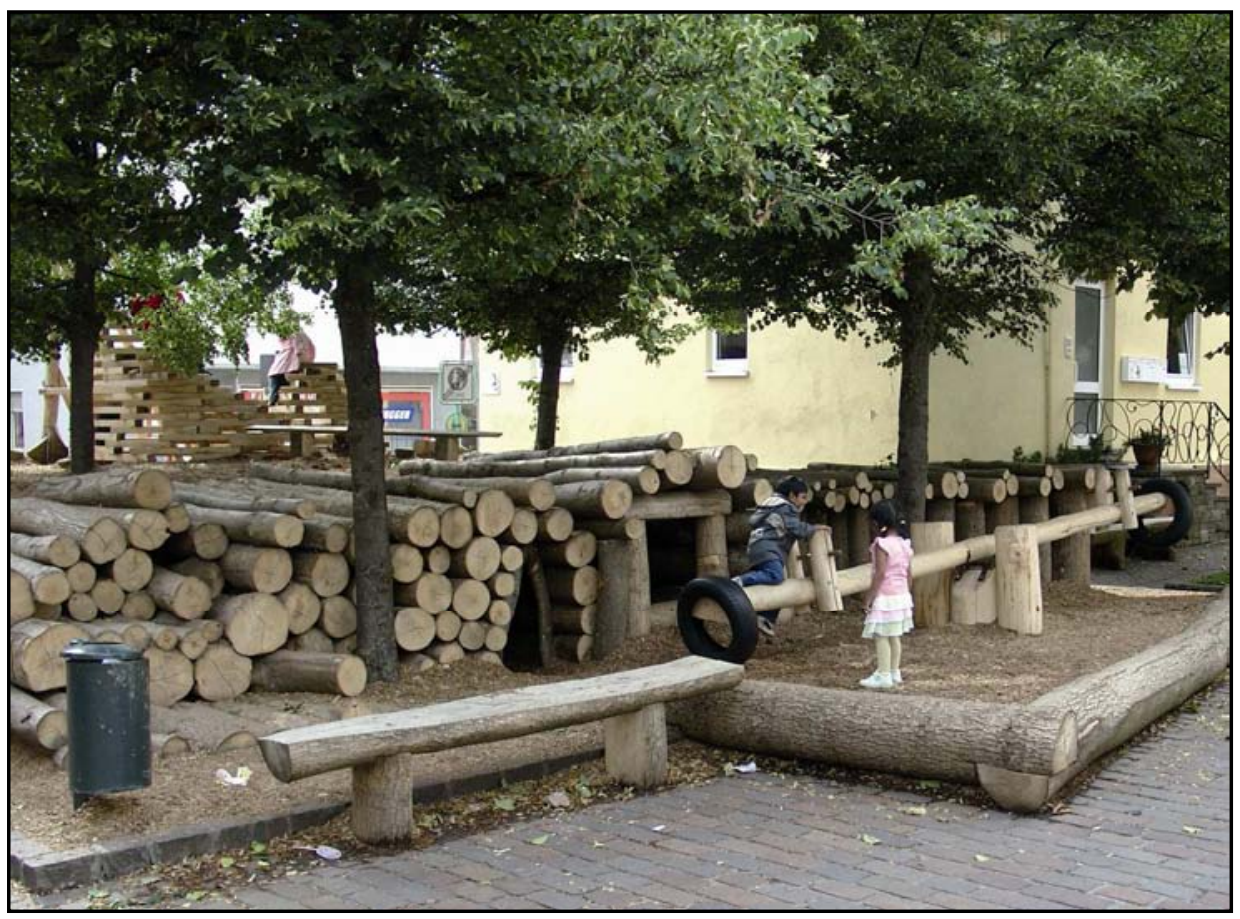

Abb. 9: Öffentlichkeitsarbeit des Forstamtes Aalen in Kooperation mit dem Berufsbildungswerk (Foto: Fleer)

Abbildung 9 zeigt eine erfolgreiche Öffentlichkeitsarbeit des Forstamtes Aalen (Ostalbkreis) in Kooperation mit dem Berufsbildungswerk, die zu einem besseren Verständnis forstbetrieblicher Arbeiten in der Region beigetragen hat. Durch spielerisches Vermitteln des Sachverhaltes nachhaltig multifunktionaler Waldwirtschaft konnten Kinder wie Erwachsene über zielgruppenangepasste Kommunikation und Bildungsarbeit erreicht werden.

Solche Maßnahmen sind zumeist nicht kurzfristig umsetzbar, können jedoch mittel- bis langfristig zielführend sein, um zu einem besseren Ansehen der Forstwirtschaft in der Öffentlichkeit und zu einem konfliktfreieren Waldbesuch beizutragen, von dem alle Interessengruppen profitieren können. 


\section{Fazit}

Eine Minderung bestehender Konfliktpotentiale zwischen der Erholungs-, der Schutz- sowie der Nutzfunktion des Waldes auf der Ostalb ist möglich. Die Herausforderung der hierfür federführend verantwortlichen Forstwirtschaft besteht dabei in der Integration vielfältiger Ansprüche an die Waldwirtschaft sowie im effizienten Ergreifen existierender Handlungsoptionen innerhalb ausreichend operationaler Rahmenvorgaben. So werden vielfach wichtige Leistungen der Forstwirtschaft für das Ökosystem und den Erholungsraum Wald durch Waldbesucher nicht erkannt oder anderen Akteuren zugeschrieben. Diesem Informationsdefizit und Kommunikationsproblem sollte mit nutzergruppenspezifischer Öffentlichkeitsarbeit abgeholfen werden.

Für die Untersuchungsregion zeigen sich Leitplanken zukunftsfähiger multifunktionaler forstlicher Nutzung des Psychotops Wald in der Entwicklung abwechslungsreicher laubholzbetonter Mischwälder, der Bestandssicherung markanter Altbäume und Naturelemente, der Erhaltung attraktiver Ausblicke in die Landschaft sowie in der Pflege eines ansprechenden beschilderten Wegenetzes. Diese Ansprüche Erholungssuchender können vielfach konfliktfrei gemeinsam mit forstbetrieblichen Zielen erreicht werden. Problemen durch Erholungsaktivitäten in naturschutzfachlich sensibel zu beurteilenden Bereichen sollte mit Sensibilisierung durch Umweltbildung sowie raum-zeitlichem Konfliktmanagement begegnet werden. Spannungen innerhalb der Gruppe der Erholungssuchenden können durch Lenkungsmaßnahmen und räumliche Entzerrung langsamer und schneller Erholungsformen entschärft werden. Diese auf den ersten Blick segregativ erscheinenden Ansätze stehen einer integrativ multifunktionalen Landnutzung nicht entgegen sondern dienen der ganzheitlich nachhaltigen Sicherung multifunktionaler Waldwirkungen auf der Gesamtfläche.

Für eine zukunftsfähige forstliche Land-Nutzung des Psychotops Wald bedeutet der integrativ multifunktionale Ansatz somit nicht ein problembehaftetes Konfliktfeld sondern die gesellschaftlich akzeptierte Basis des nachhaltigen Miteinanders der Nutz-, Schutz- und Sozialfunktion im Dreieck nachhaltiger Waldnutzung. 


\section{Literatur und Quellen}

Ammer, U. u. Pröbstl, U. (1991): Freizeit und Natur, Probleme und Lösungsmöglichkeiten einer ökologisch verträglichen Freizeitnutzung. Pareys Studientexte 72. Berlin/Hamburg.

Fleer, I. (2008): Der Wald als Psychotop: Konkurrenz und Konfliktfeld zur Nutzund Schutzfunktion? Diplomarbeit am Zentrum für Naturschutz an der Biologischen Fakultät sowie an der Fakultät für Forstwissenschaften und Waldökologie der Georg-August-Universität Göttingen.

Friedhoff, T. (2006): Untersuchung der Erholungsfunktion des Waldes als Beitrag zur nachhaltig wertleistungsoptimierten Waldwirtschaft im waldreichen ländlichen Raum Ostalb. Masterarbeit an der Fakultät für Forstwissenschaften und Waldökologie der Georg-August-Universität Göttingen.

Mann, C. (2006): Konflikte in Erholungsgebieten - Ursachen, Wirkungen und Lösungsansätze. Verlag Kessel. Remagen.

Meuser, M. u. Nagel, U. (1991): ExpertInneninterviews - vielfach erprobt, wenig bedacht. In: Garz, D. u. Kraimer, K. (Hrsg.): Qualitativ-empirische Sozialforschung. Konzepte, Methoden, Analysen. Westdeutscher Verlag. Opladen. S. 441-471.

Riegert, C. u. Kempa, D. (2008): Naturschutz und Naherholung im urbanen Raum am Beispiel der Stadt Heidenheim. In: Reeh, T. u. Ströhlein, G. (Hrsg.): Natur erleben und Raum inszenieren. ZELTForum, Göttinger Schriften zu Landschaftsinterpretation und Tourismus, Bd. 4., Universitätsverlag Göttingen. Göttingen.

Riegert, C., Kempa, D. u. Bürger-Arndt, R. (2009): Nachhaltig multifunktionale Forstwirtschaft - Rahmenvorgaben einer wertleistungsoptimierten Waldnutzung. In: Fakultät für Forst- und Umweltwissenschaften der Universität Freiburg (FVA) (Hrsg.): Freiburger Forstliche Forschung, H. 81, S. 65-96.

Stölb, W. (2005): Waldästhetik. Über Forstwirtschaft, Naturschutz und die Menschenseele. Verlag Kessel. Remagen-Oberwinter. 
Künstliche Beschneiung als Anpassungsstrategie an die Klimaerwärmung und die Nachfrage im Wintertourismus? Eine Fallstudie zur Sicht der Touristen

David Gallati 


\begin{abstract}
Technical snowmaking has become an important adaptation strategy in the Alps to climate change and seasonal weather variability as well as to satisfy customer's increasing requirements. This study aims at estimating the motives of destination visit as well as to assess the relevance of technical snowmaking for winter tourism and the attractiveness of a destination focussing the guests' motives of travelling and their attitude towards snowmaking. The results of an empirical study in the three Swiss winter sport destinations Davos, Scuol and Braunwald show large regional and seasonal differences concerning the tourists' motives of visit and the attitude towards technical snowmaking. The landscape and a large choice of tourist activities like for example skiing, hiking or biking are key factors for the success of tourism in the Swiss mountains. However, snowmaking is neither the only nor the crucial factor affecting the attractiveness of a destination, nor does it constitute the central motive for the choice of a certain destination. Even though, snowmaking is increasingly accepted and the equipment even expected more and more as a basic setting.
\end{abstract}

\title{
Einleitung
}

Die Auswirkungen der globalen Klimaerwärmung wurden in den letzten Jahren zu einem viel diskutierten Thema in einer breiten Öffentlichkeit. Gemäß des internationalen Ausschusses für Klimawandel (IPCC) ist die durchschnittliche Temperatur an der Erdoberfläche in den vergangenen 100 Jahren um $0,74^{\circ} \mathrm{C}$ gestiegen (Trenberth u. Jones 2007, S. 235). In der zweiten Hälfte der 1980er Jahre vollzog sich in Mitteleuropa ein deutlicher Sprung hin zu wärmeren Wintern. Obschon es auch in Zukunft immer wieder kalte und schneereiche Winter geben wird, bleibt die Tendenz hin zu wärmeren Wintern bestehen (vgl. Bader u. Bantle 2004). Die globale Erwärmung folgt bestimmten regionalen und temporalen Mustern. So erfolgte über die letzten Dekaden die stärkste Erwärmung über den kontinentalen Landmassen (vgl. Trenberth u. Jones 2007, S. 236). In der Schweiz stieg die durchschnittliche Jahrestemperatur über die vergangenen 30 Jahre mit $0,4^{\circ}$ bis $0,6^{\circ} \mathrm{C}$ pro Dekade bedeutend stärker als im globalen Mittel $\left(0,1^{\circ}\right.$ bis $\left.0,2^{\circ} \mathrm{C}\right)$. Die Ursache für diese Entwicklung wird in der Modifizierung der nordatlantischen Oszillation sowie der Abnahme der Schneeflächen in den Alpen und der damit veränderten Albedo (Sonnenrückstrahlungsvermögen) angenommen (vgl. Neu 2002, S. 11). Die erwartete Wintertemperaturzunahme bis 2050 wird im Vergleich zu 1990 auf plus $1.8^{\circ} \mathrm{C}$ geschätzt (vgl. Frei et al. 2007, S. 14).

Dieser zukünftige Temperaturanstieg wird unter anderem starke Auswirkungen auf den alpinen Tourismus haben. Die Klimaerwärmung kann sowohl das Han- 
deln der Touristen als auch die Voraussetzungen in den Destinationen beeinflussen (vgl. Müller et al. 2007, S. 80). Der im Zusammenhang mir der Klimaerwärmung erwartete Anstieg von Starkniederschlägen und anderen Wetterextremen wird dazu führen, dass die Erreichbarkeit alpiner Destination durch vermehrte Gefährdung der Zufahrtswege beeinträchtigt wird. Schmelzende Gletscher im Sommer und verminderte Schneesicherheit im Winter werden sich auf die Attraktivität der Tourismusregionen auswirken. Insbesondere der Wintertourismus, ein wichtiges ökonomisches Standbein in vielen Regionen der Alpen, dürfte besonders stark von steigenden Temperaturen betroffen sein (vgl. Abegg 1996; Breiling et al. 1997; Scott u. Dawson 2007; Steiger 2007).

Mit den höheren durchschnittlichen Wintertemperaturen steigt auch die natürliche Untergrenze der schneesicheren Höhenregionen und damit die Wahrscheinlichkeit von Schneemangel in tiefer liegenden Gebieten. Die Schneebedeckung hat unterhalb von $1300 \mathrm{~m}$ ü. M. seit 1980 bereits statistisch signifikant abgenommen (vgl. Laternser u. Schneebeli 2003). Eine etablierte Regel für die Definition von Schneesicherheit stammt von Witmer (1984), welche in den Arbeiten von Abegg (1996) und Bürki (2000) sowie in der aktuellen OECD-Studie (Agrawala 2007) wieder aufgenommen und erweitert wurde. Gemäß dieser Regel kann eine Region als schneesicher bezeichnet werden, wenn in 7 von 10 Jahren eine für den Skisport ausreichende Schneebedeckung von $30 \mathrm{~cm}$ während wenigstens 100 Tagen zwischen dem 16. Dezember und 15. April vorhanden ist. Ein Anstieg der mittleren Wintertemperaturen um $4^{\circ} \mathrm{C}$ - was bis Ende dieses Jahrhunderts als wahrscheinliches Szenario gilt - würde diese Untergrenze der schneesicheren Gebiete von heute ca. $1200 \mathrm{~m}$ ü. M. auf ca. $1800 \mathrm{~m}$ ü. M. in den Zentralalpen und auf ca. 2100 $\mathrm{m}$ ü. M. in den Südalpen anheben, was mit gravierenden Folgen für die tiefer gelegenen Skigebiete verbunden wäre (vgl. Abegg et al. 2007). Meier (1998, S. 80) schätzt die jährlichen wirtschaftlichen Verluste im schweizerischen Wintertourismus aufgrund der Klimaerwärmung auf 1.7 bis 2.2 Milliarden Schweizer Franken.

Vor diesem Hintergrund erstaunt die steigende Anwendung von Anpassungsstrategien in den alpinen Skiregionen nicht. Es gibt sowohl technische Anpassungsstrategien (künstliche Beschneiung, Terrainausebnung, bodenunabhängige Transportmittel ${ }^{1}$, Erschließung höher gelegener Gebiete) als auch strukturelle Anpassungsstrategien (Angebotserweiterungen, Vier-Jahreszeiten-Tourismus).

Insbesondere die künstliche Beschneiung ist eine weit verbreitete Anpassungsmaßnahme in den Alpen, um das Wintersportangebot von wärmeren Temperaturen und saisonalen Witterungsvariabilitäten unabhängiger zu gestalten sowie den steigenden Qualitätsbedürfnissen der Wintersportler gerecht zu werden. In über 90\% der alpinen Skigebiete sind bereits Beschneiungsanlagen installiert (vgl. CIPRA 2006, S. 6). Gemäß Seilbahnen Schweiz (2008, S. 19) können aktuell 33\% der gesamten Pistenflächen in der Schweiz künstlich beschneit werden, Tendenz steigend. Im Vergleich dazu sind in Italien 70\%, in Österreich 59\% und in Frank-

${ }^{1}$ Z.B. Sessellift anstatt Skilift 
reich 19\% der Pistenfläche mit Beschneiungsanlagen ausgerüstet. Inwiefern mit künstlicher Beschneiung die natürliche Schneegrenze und Saisonlänge gesteuert werden kann, ist schwierig abzuschätzen und oftmals nicht generalisierbar, da dies stark von Faktoren wie den regionalen, mikroklimatischen Bedingungen, der Exposition (vgl. Teich et al. 2007) und technischen Entwicklungen der Beschneiungsanlagen (vgl. Steiger 2006) abhängig ist. Steiger (2007) führt aus, dass künstliche Beschneiung zurzeit noch die gewünschte Saisonlänge sicherstellen, nicht aber die zukünftige Saisonverkürzung in tieferen Lagen kompensieren kann.

Zahlreiche Studien erörtern die Motive der Tourismusverantwortlichen für den Einsatz von Kunstschnee (Mayer et al. 2007; Pröbstl 2006; Scott et al. 2003; Elsasser u. Bürki 2002; Bürki 2000; Abegg 1996; Studer u. Christoffel 1990) sowie die Auswirkungen wärmerer Winter auf die Skiindustrie (Abegg et al. 2007; Mayer et al. 2007; Scott u. Jones 2005). Gewichtige Gründe für den Einsatz von Kunstschnee stellen die kurzfristige, mikroökonomische Risikoabsicherung der Bergbahnunternehmen und die Gewährleistung der Ertragsleistung der tourismusabhängigen Unternehmen vor dem Hintergrund zunehmend wärmerer Winter und immer größerer Investitionen dar (vgl. Scott et al. 2003; Job 2005; Peck 2005). Die künstliche Beschneiung garantiert gute Schneebedingungen während der ganzen Wintersaison und erhöht damit die durchschnittliche Auslastung der touristischen Infrastruktur (vgl. Pröbstl 2006, Peck 2005, Fauve et al. 2002). Wintersportler und deren Ausrüstung verlangen nach immer besser präparierten und sichereren Pisten (vgl. Mayer et al. 2007; Fauve et al. 2002). Die Durchführung von Trainings und Wettbewerben im Hochleistungssport, welcher hohe Ansprüche an die Schneebedingungen stellt, sollen mit Hilfe von künstlicher Beschneiung zuverlässig durchgeführt werden können und somit zu einem positiven Image der Destination beitragen (vgl. Pröbstl 2006; Fauve et al. 2002). Des Weiteren wird die künstliche Beschneiung von den Tourismusverantwortlichen oft als wichtiges Kriterium im internationalen Destinationswettbewerb angesehen (vgl. Mayer et al. 2007; Job 2005; Abegg 1996).

Unter Beachtung dieser ökonomischen, klimatischen und touristischen Faktoren ist davon auszugehen, dass der Einsatz von Kunstschnee im Alpenraum in Zukunft nach wie vor zunehmen wird. Deshalb ist es wichtig, bei strategischen Überlegungen der Tourismusverantwortlichen nebst den möglichen Auswirkungen der Klimaerwärmung auch die Präferenzen der Touristen und deren Wahrnehmung von Kunstschnee zu kennen.

In weit geringerem Maße wurde bis anhin Forschung zur Einstellung der Touristen gegenüber Kunstschnee und Klimaerwärmung betrieben. Die Ergebnisse der Umfrage von Studer und Christoffel (1990) in vier schweizerischen Skidestinationen förderte eine breite Ablehnung gegenüber großflächiger Beschneiung zu Tage, obschon die Mehrheit ein punktueller Einsatz von Beschneiungsanlagen durchaus befürwortete. Bürkis Studie (2000) in fünf Skidestinationen in den Kantonen Ob- und Nidwalden (Schweiz) zeigte, dass knapp die Hälfte der befragten Touristen die künstliche Beschneiung als wichtige Anpassungsmaßnahmen unter 
künftig wärmeren Bedingungen ansieht. Zudem wurde offenbar, dass die Wahrnehmung der Klimaerwärmung und der Schneesicherheit einen Einfluss auf das Reiseverhalten der Touristen haben kann. Légeret (2006) konnte mit einer Studie in den Schweizer Alpen eine gewisse Zahlungsbereitschaft der Touristen für Schneesicherheit nachweisen.

Die vorliegende Studie legt die Resultate einer Befragung dar, die durchgeführt wurde, um die Besuchsmotive eines Ortes sowie die Relevanz der künstlichen Beschneiung und der Schneesicherheit als Attraktivitätspunkt einer Destination zu eruieren.

\section{Methoden}

Mit einer Gästebefragung in den Destinationen Braunwald, Davos und Scuol wurden Informationen bezüglich der Wahrnehmungsmuster, Reaktionen und Entscheidungsprozesse im Kontext der künstlichen Beschneiung und der Klimaerwärmung erhoben. Die aufgrund der Fragestellung verlangte Information beruht hauptsächlich auf subjektiven Normvorstellungen, Meinungen und Einstellungen. Diese Art von Information kann gemäß Hermann et al. (2004, S. 97) nur über verbale Äußerungen der untersuchten Menschen erschlossen werden.

Der Vergleichbarkeit der Befragungsresultate halber bot sich eine stark strukturierte (standardisierte) Kommunikationsform als geeignete Befragungsmethode an, weil damit die Voraussetzungen für jeden Befragten dieselben sind. Auch können die Daten von mehreren Personen erhoben werden, da keine Abhängigkeit von der Person des Forschers besteht und zudem ein relativ hohes Maß an Neutralität des Interviewers gesichert ist. Die Stichprobe wurde zufällig aus der Gesamtheit der während der Interviewtage im Sommer 2006 und Winter 2006/07 anwesenden Touristen in den erwähnen Destinationen ausgesucht. Die Befragung fand an jedem Ort während zwei Tagen zu drei verschiedenen Zeitpunkten statt, um eine breite Datengrundlage zu erhalten und sowohl saisonale als auch regionale Unterschiede erfassen zu können. Insgesamt wurden 261 Touristen in Braunwald, 261 in Davos und 269 in Scuol befragt. Die Befragungsorte wurden aufgrund ihrer unterschiedlichen Angebotsstruktur und klimatischen Gegebenheiten ausgesucht, um damit verschieden Gästestrukturen abgedeckt zu haben (vgl. Tab. 1).

Der verwendete standardisierte Fragebogen besteht aus vier Hauptteilen mit sowohl offenen (keine vorgegebenen Antwortmöglichkeiten) als auch geschlossenen (Auswahl vorgegebener Antwortmöglichkeiten) Fragen. Der erste Teil beinhaltet Fragen über Ausgangsort, Anreise und Aufenthalt. Im zweiten Teil wurden die Attraktivitäten der Destination sowie die Besuchsmotive erfasst. Wintertourismus und die Wahrnehmung künstlicher Beschneiung sind Inhalt des dritten Teils. Einige der Fragen wurden aus älteren Studien von Studer u. Christoffel (1990, S. 133) und Bürki (2000, S. 206) übernommen, um eine gewisse Vergleich- 
barkeit zu gewähren und zeitliche Entwicklungen aufzeigen zu können. Im vierten Teil werden soziodemographische Merkmale (Alter, Beruf u.a.) abgefragt.

Tab. 1: Wirtschaftliche, touristische und klimatische Daten der Fallstudienorte (Quelle: nach Teich et al., 2007)

\begin{tabular}{|c|c|c|c|}
\hline Ort & $\begin{array}{l}\text { Davos } \\
\text { Kanton Graubünden, } \\
\text { Schweiz }\end{array}$ & $\begin{array}{l}\text { Scuol } \\
\text { Kanton Graubünden, } \\
\text { Schweiz }\end{array}$ & $\begin{array}{l}\text { Braunwald } \\
\text { Kanton Glarus, } \\
\text { Schweiz }\end{array}$ \\
\hline Höhe & $1.560 \mathrm{~m}$ ü. M. & $1.250 \mathrm{~m}$ ü. M. & 1.256 m ü. M. \\
\hline Einwohner & 12.621 & 2.400 & 349 \\
\hline Wirtschaft & $\begin{array}{l}\text { Winter- und Sommertouris- } \\
\text { mus: Schneesport, Wan- } \\
\text { dern; Kongresstourismus; } \\
\text { Landwirtschaft; Dienstleis- } \\
\text { tungen: Forschung, Ge- } \\
\text { sundheit }\end{array}$ & $\begin{array}{l}\text { Winter- und Sommer- } \\
\text { tourismus: } \\
\text { Schneesport, Wan- } \\
\text { dern, Biken; Well- } \\
\text { ness; Landwirt- } \\
\text { schaft; Elektrizitäts- } \\
\text { industrie }\end{array}$ & $\begin{array}{l}\text { Winter- und Sommer- } \\
\text { tourismus: } \\
\text { Schneesport, Wan- } \\
\text { dern, Klettern; Land- } \\
\text { wirtschaft }\end{array}$ \\
\hline $\begin{array}{l}\text { Anzahl } \\
\text { Übernacht- } \\
\text { ungen }\end{array}$ & $\begin{array}{l}2.1 \text { Millionen (Jahr 2003/ } \\
\text { 2004); Verhältnis: Winter } \\
60 \% \text {, Sommer 40\% }\end{array}$ & $\begin{array}{l}422.678 \text { (Jahr } \\
\text { 2005/2006); Verhält- } \\
\text { nis: Winter } 50 \% \text {, } \\
\text { Sommer } 50 \%\end{array}$ & $\begin{array}{l}67.849 \text { (Jahr } \\
\text { 2005/2006); Verhält- } \\
\text { nis: Winter 59\%, } \\
\text { Sommer 41\% }\end{array}$ \\
\hline $\begin{array}{l}\text { Skiregio- } \\
\text { nen }\end{array}$ & $\begin{array}{l}\text { Parsenn/Gotschna (1.179- } \\
2.844 \mathrm{~m} \text { ü. M.), Jakobshorn } \\
\text { (1.540-2.590 m ü. M.), } \\
\text { Pischa (1.800-2.483 m ü. } \\
\text { M.), Rinerhorn (1.454-2.490 } \\
\text { m ü. M.), Madrisa (1.124- } \\
\text { 2.602 m ü. M.) }\end{array}$ & $\begin{array}{l}\text { Motta Naluns (1.250- } \\
2.785 \text { m ü. M.) }\end{array}$ & $\begin{array}{l}\text { Braunwald (1.300- } \\
1.904 \text { m ü. M.) }\end{array}$ \\
\hline $\begin{array}{l}\text { Pistenkilo- } \\
\text { meter }\end{array}$ & Total $284 \mathrm{~km}$ & $80 \mathrm{~km}$ & $25 \mathrm{~km}$ \\
\hline $\begin{array}{l}\text { Prozentan- } \\
\text { teil } \\
\text { beschnei- } \\
\text { barer Pis- } \\
\text { tenfläche }\end{array}$ & $\begin{array}{l}\text { Parsenn/Gotschna: } 20 \% \text {, } \\
\text { Jakobshorn: } 45 \%\end{array}$ & $30 \%$ & $3 \%$ \\
\hline Klima & $\begin{array}{l}\text { Jährliche Durch- } \\
\text { schnittstemp.: +2.8 } \mathrm{C} \text {, Nie- } \\
\text { derschlag: Übergangszone } \\
\text { von feuchten Nord- zu tro- } \\
\text { ckenen Zentralalpen }\end{array}$ & $\begin{array}{l}\text { Jährliche Durch- } \\
\text { schnittstemp.: }+6^{\circ} \mathrm{C} \text {, } \\
\text { Niederschlag: konti- } \\
\text { nentale inner-alpine } \\
\text { Trockenzone }\end{array}$ & $\begin{array}{l}\text { Jährliche Durch- } \\
\text { schnittstemp.: }+5^{\circ} \mathrm{C} \text {, } \\
\text { Niederschlag: nieder- } \\
\text { schlagsreiche Zone } \\
\text { der Alpennordseite }\end{array}$ \\
\hline
\end{tabular}

Die empirisch gewonnenen Daten wurden für die anschließende Analyse digitalisiert. Die Antworten der geschlossenen Fragen konnten direkt in Variablen transferiert werden, während die Resultate der offenen Fragen erst klassifiziert und 
anschließend in Variablen umgesetzt wurden. Die Klassifizierung geschah nach Häufigkeit der Nennung bestimmter Stichworte. Die meisten Daten sind nominal und nur einige wenige ordinal skaliert. Hypothesen wurden formuliert, um den Einfluss persönlicher Merkmale der Gäste auf die Akzeptanz von Kunstschnee zu analysieren. Aufgrund des tiefen Skalenniveaus lassen sich die Hypothesen vernehmlich mit dem Chi-Quadrat-Signifikanztest überprüfen.

Die Analyse der gewonnen Daten ermöglicht es, die Größe des Einflusses von Kunstschnee für die Attraktivität einer Destination qualitativ abzuschätzen und wesentliche Besuchsmotive in Unterscheidung der Destination und der Saison zu eruieren.

\section{Resultate}

In Abhängigkeit von der Saison und der Destination zeigen die Resultate große Differenzen zum einen bezüglich der Erwartungen und Bedürfnisse der Touristen an die gewählte Destination sowie zum anderen bezüglich der Einstellung gegenüber Kunstschnee und Schneesicherheit. Jede der untersuchten Destinationen zeichnet sich durch eine spezifische Gästestruktur aus, welche sich in den Einstellungen und Präferenzen der Gäste ausdrückt. Die Verallgemeinerbarkeit der Ergebnisse und die Übertragbarkeit der Erkenntnisse auf andere alpinen Destinationen sind damit allerdings nur bedingt möglich. Ebenso wenig können ein/e typische/r Wintersportler/in oder ein typischer Gast der Schweizer Alpen charakterisiert werden. Vielmehr muss der orts- und saisonspezifische Kontext der Befragungsergebnisse betont und für die Darstellung und Interpretation der Ergebnisse berücksichtigt werden. Im Folgenden werden die unterschiedlichen Gästestrukturen der drei Destinationen skizziert.

\section{Gästestruktur}

In Davos unterschied sich die Altersstruktur der befragten Gäste zwischen den einzelnen Befragungsblöcken beträchtlich. Während im Sommer ein Großteil $(48 \%)$ der Befragten über 60 Jahre alt war, waren im Winter zur Vorsaison 55\% und zur Hauptsaison 35\% der Befragten unter 35 Jahre alt. Der Anteil ausländischer Gäste war in Davos deutlich höher als in Braunwald und Scuol. Von den im Sommer befragten Gästen kamen 65\% aus dem Ausland, davon 80\% aus Deutschland. Im Winter betrug der Anteil ausländischer Gäste 40\%. Der tatsächliche Ausländeranteil dürfte in der Gästebefragung unterrepräsentiert gewesen sein, weil zahlreiche ausländische Gäste aufgrund sprachlicher oder organisatorischer Probleme (Gruppenreisen) nicht befragt werden konnten. Etwa die Hälfte der befragten Übernachtungsgäste wohnte in Davos in Hotels, 30\% nächtigten in einer Ferienwohnung oder in einem Ferienhaus. In der Winterhauptsaison im Februar war die Parahotellerie mit 50\% stärker vertreten als die Hotellerie (40\%). 
Die Altersstruktur der befragten Gäste in Scuol unterschied sich saisonal kaum. Lediglich der Anteil der über 70-jährigen Gäste war im Sommer etwas höher als der Anteil der 40- bis 60-jährigen Gäste, in der Wintervorsaison etwas niedriger. Der Anteil ausländischer Gäste betrug im Sommer etwas über 30\% und im Winter etwa $20 \%$. Von den Übernachtungsgästen wohnten im Sommer $58 \%$ in Hotels, $27 \%$ in Ferienhäusern und Ferienwohnungen und 10\% auf dem Campingplatz. Im Winter wurden bevorzugt Ferienhäuser und Ferienwohnungen als Unterkunft gewählt, zur Vorsaison 50\% und zur Hauptsaison $60 \%$.

In Braunwald variierte die Altersstruktur der befragten Gäste saisonal kaum. Die Altersgruppe der 40- bis 60-jährigen war zu allen Zeitpunkten am stärksten vertreten. Der Anteil ausländischer Gäste war generell gering und mit 14\% im Sommer am höchsten. Die meisten Gäste nächtigten in einem Ferienhaus oder in einer Ferienwohnung, gefolgt von Hotels und Gruppenunterkünften (u.a. Berghaus, Jugendherberge oder Bergwander-Hütte). Dabei nächtigten durchschnittlich $44 \%$ der Gäste in eigenen Ferienhäusern oder -wohnungen, im Sommer sogar $72 \%$. Diese Zahlen deuten auf eine große Anzahl an Stammgästen in Braunwald hin, lagen doch die Eigentumsanteile in den beiden anderen Destinationen deutlich niedriger (26\% in Davos, $21 \%$ in Scuol).

\section{Einstellung gegenüber Kunstschnee}

Die folgenden Resultate ergaben sich aus der geschlossenen Frage nach der Einstellung gegenüber dem Einsatz von Kunstschnee (dafür, dagegen, unentschieden) sowie der anschließend verlangten (offenen) Begründung. In der Sommerbefragung war die Mehrheit der befragten Gäste gegen den zunehmenden Einsatz künstlicher Beschneiung. Die höchste Ablehnung wurde in Braunwald erreicht, wo sich $46 \%$ dagegen und nur 35\% dafür aussprachen (vgl. Abb. 1). Die am häufigsten genannten Argumente dagegen waren Umweltschutzgründe, Ressourcenverschwendung (Wasser, Strom) und die Meinung, dass anthropogene Eingriffe in den Naturhaushalt generell unterlassen werden sollten.

Die Resultate der Winterbefragung fielen regional unterschiedlich aus. Während sich in Braunwald nach wie vor eine Mehrheit gegen die Anwendung von Kunstschnee aussprach, unterstützte die Mehrheit der Gäste der beiden hoch gelegenen Destinationen Davos und Scuol die künstliche Beschneiung. Die höchste Zustimmung wurde in Scuol während der Wintervorsaison erreicht $(60 \%)$. Diese Position wurde mehrheitlich mit dem Argument der gewährleisteten Schneesicherheit und Pistenqualität während der gesamten Saison unabhängig von der Witterung begründet. Des Weiteren wurde die künstliche Beschneiung oft als Notwendigkeit im heutigen Kontext der Klimaerwärmung und des internationalen Wettbewerbsdruckes angesehen. Für viele scheint sie die Lösung zur Erhaltung der regionalwirtschaftlichen Existenz der alpinen Destinationen zu sein. Dieses Argument wurde oft verwendet, um sich zwar persönlich von der künstlichen Beschneiung zu distanzieren aber gleichzeitig Verständnis für deren Anwendung 
aufzubringen: „Ich bin eigentlich eher gegen die Anwendung von Kunstschnee, aber es ist wichtig für das Überleben der Region."

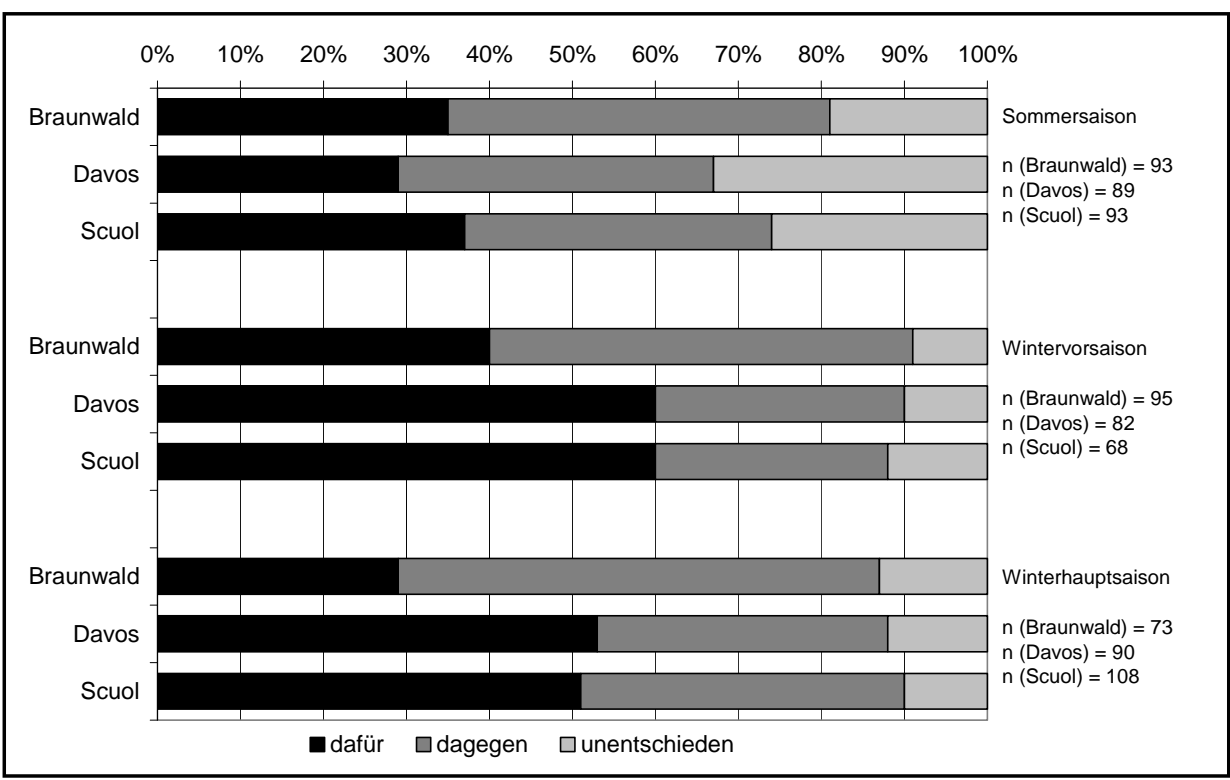

Abb. 1: Anteil der Befürworter/Gegner/Unentschiedenen von Kunstschnee in Prozent nach Saison und Destination $(n=791)$.

Die regional und saisonal unterschiedliche Einstellung gegenüber Kunstschnee legt nahe, dass diese von der Gästestruktur und somit von gewissen Eigenschaften der einzelnen Gäste abhängig ist. Tabelle 2 gibt einen Überblick über die verschiedenen getesteten Hypothesen, die zur Analyse des Einflusses der Eigenschaften der Gäste auf die Akzeptanz von Kunstschnee aufgestellt wurden. Dabei zeigten die Ausübung von Wintersport und die Aufenthaltsdauer vor Ort einen gewissen Einfluss auf die Akzeptanz von Kunstschnee, wobei die (positive) Korrelation zwischen diesen Merkmalen und der Akzeptanz relativ schwach war (Spearman Correlation). Der Besitz von Ferienimmobilien bewirkte ebenfalls einen Trend zur verstärkten Akzeptanz, wobei dieser statistisch nicht signifikant erhärtet werden konnte. 
Tab. 2: Eigenschaften der befragten Gäste und deren Beitrag an die Akzeptanz von Kunstschnee

\begin{tabular}{|c|c|c|c|}
\hline Variable (Skalenniveau) & t-Statistik & P-Wert & $\begin{array}{l}\text { Beurteilung des Einflusses } \\
\text { der Variable auf Akzeptanz }\end{array}$ \\
\hline $\begin{array}{l}\text { Geschlecht (dichotome } \\
\text { Nominalskala) }\end{array}$ & $\begin{array}{l}\text { Pearson Chi- } \\
\text { Quadrat: } 0.026\end{array}$ & 0.871 & $\begin{array}{l}\text { Kein signifikanter Zusam- } \\
\text { menhang }\end{array}$ \\
\hline $\begin{array}{l}\text { Ausübung von Wintersport } \\
\text { (dichotome Nominalskala) }\end{array}$ & $\begin{array}{l}\text { Pearson Chi- } \\
\text { Quadrat: } 7.838 \text {; } \\
\text { Spearman Korrela- } \\
\text { tion: } 0.131\end{array}$ & $\begin{array}{l}0.005 \\
0.005\end{array}$ & $\begin{array}{l}\text { Signifikanter Zusammen- } \\
\text { hang, aber schwache Korre- } \\
\text { lation }\end{array}$ \\
\hline $\begin{array}{l}\text { Anzahl Schneesporttage } \\
\text { pro Jahr (Ordinalskala) }\end{array}$ & $\begin{array}{l}\text { Pearson Chi- } \\
\text { Quadrat: } 2.605\end{array}$ & 0.457 & $\begin{array}{l}\text { Kein signifikanter Zusam- } \\
\text { menhang }\end{array}$ \\
\hline Alter (Ordinalskala) & $\begin{array}{l}\text { Pearson Chi- } \\
\text { Quadrat: } 2.938\end{array}$ & 0.401 & $\begin{array}{l}\text { Kein signifikanter Zusam- } \\
\text { menhang }\end{array}$ \\
\hline $\begin{array}{l}\text { Eigentümer von Immobi- } \\
\text { lien vor Ort (dichotome } \\
\text { Nominalskala) }\end{array}$ & $\begin{array}{l}\text { Pearson Chi- } \\
\text { Quadrat: } 1.271 \text {; } \\
\text { Spearman Korrela- } \\
\text { tion: }-0.056\end{array}$ & $\begin{array}{l}0.260 \\
0.261\end{array}$ & $\begin{array}{l}\text { Schwacher Zusammenhang } \\
\text { und schwache Korrelation }\end{array}$ \\
\hline $\begin{array}{l}\text { Aufenthaltsdauer } \\
\text { (Ordinalskala) }\end{array}$ & $\begin{array}{l}\text { Pearson Chi- } \\
\text { Quadrat: } 5.079 \\
\text { Spearman Korrela- } \\
\text { tion: }-0.119\end{array}$ & $\begin{array}{l}0.024 \\
0.024\end{array}$ & $\begin{array}{l}\text { Signifikanter Zusammen- } \\
\text { hang, aber schwache Korre- } \\
\text { lation }\end{array}$ \\
\hline $\begin{array}{l}\text { Besuchshäufigkeit } \\
\text { (Ordinalskala) }\end{array}$ & $\begin{array}{l}\text { Pearson Chi- } \\
\text { Quadrat: } 2.797 \\
\end{array}$ & 0.592 & $\begin{array}{l}\text { Kein signifikanter Zusam- } \\
\text { menhang }\end{array}$ \\
\hline
\end{tabular}

Besuchsmotive und Attraktionen

Wie die Gästestruktur variierten auch die Motive für die Anreise zwischen den betrachteten Destinationen und Zeitpunkten (vgl. Abb. 2). Die Kategorisierung und Auswertung der offenen Frage nach den beiden wichtigsten Gründen für die Anreise sowie der geschlossenen Frage nach den unternommenen Aktivitäten lieferten folgende Resultate: Im Sommer war die landschaftliche Schönheit das wichtigste Motiv. 36\% der Gäste in Davos waren deswegen angereist, in Scuol waren es 22\% und in Braunwald 18\%. Des Weiteren hat beinahe ein Drittel der Touristen Davos aufgrund der Ausübung bestimmter Aktivitäten besucht, wobei das Wandern (60\%) und das Spazieren (28\%) den größten Anteil ausmachten. $11 \%$ der Besucher Braunwalds kamen, um zu wandern (63\%), zu spazieren (13\%) oder zu klettern (12\%). Das Wandern (61\%) und das Biken (13\%) waren die Hauptaktivitäten von insgesamt 28\% der Gäste in Scuol.

Zur Wintervorsaison wurde die Erreichbarkeit zu einem wichtigen Besuchsmotiv, da die meisten Gäste zu dieser Zeit nur für kurze Zeit anreisten. 14\% der Besucher kamen aufgrund der guten Erreichbarkeit nach Braunwald. Je länger die 
Anfahrt zu einer Destination dauerte, desto weniger wichtig war dieses Motiv (Davos 6\%, Scuol 3\%) für deren Wahl.

Das Skigebiet stellte das entscheidende Motiv in Davos (20\%) und Scuol $(21 \%)$ zur Winterhauptsaison dar, während in Braunwald nach wie vor andere Motive wie das Wandern und Spazieren eine übergeordnete Rolle spielten. Von Bedeutung in allen Destinationen waren die Gewohnheit des alljährlichen Skiurlaubs vor Ort und die Verfügbarkeit einer attraktiven Unterkunft.

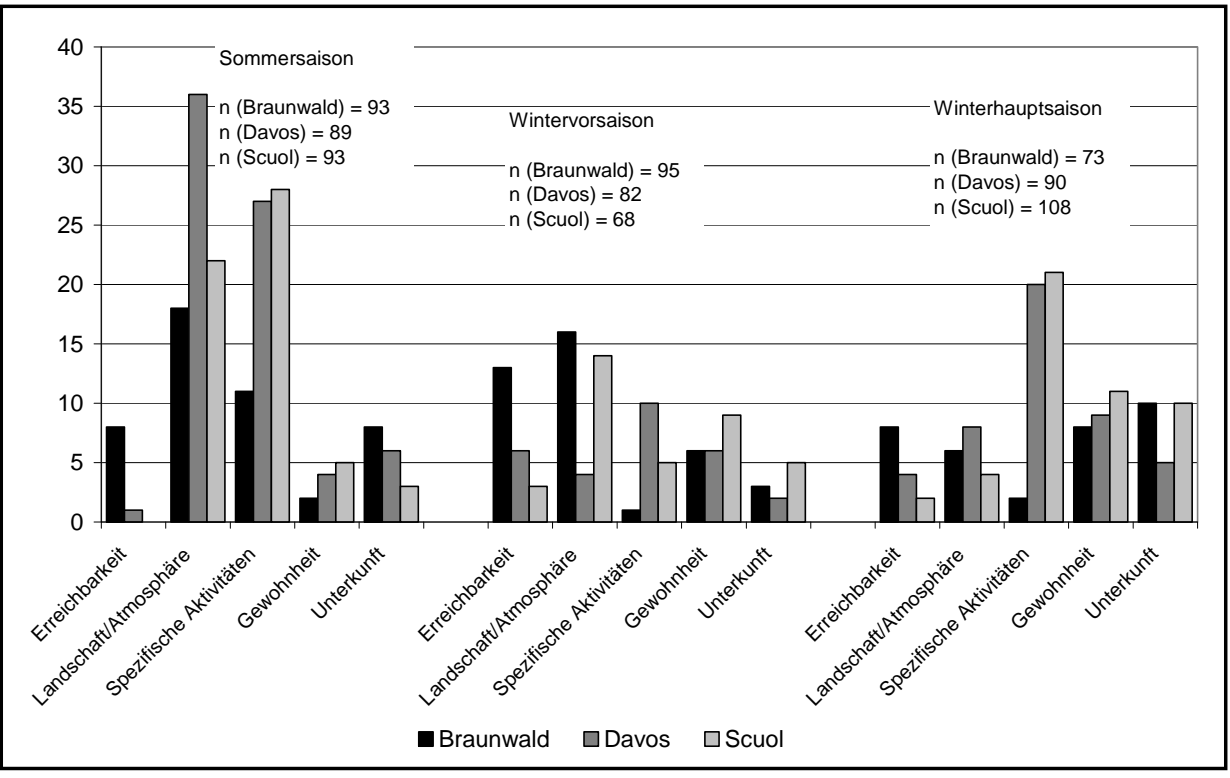

Abb. 2: Die meistgenannten Besuchmotive in den untersuchten Destinationen zur Sommer-, Wintervor- und Winterhauptsaison. Die Kategorisierung der Antworten auf die offene Frage nach den zwei wichtigsten Motiven für die Anreise geschah nach Häufigkeit der Nennung gewisser Schlagworte.

\section{Schneesicherheit als Besuchsmotiv}

Im Zusammenhang mit der künstlichen Beschneiung interessiert insbesondere die Schneesicherheit als Besuchsmotiv. Wie in Tabelle 3 gezeigt wird, beantworteten die geschlossene Frage, ob ihnen die Schneesicherheit vor Ort wichtig ist (ja, nein, weiß nicht), zwischen 69\% (Braunwald) und 84\% (Scuol) der befragten Gäste mit „ja“. Der Anteil innerhalb der Gruppe „Wintersportler“ ist dabei noch deutlich höher. Bei der offenen Frage nach den zwei wichtigsten Gründen für die Anreise hingegen, gaben nur ganz wenige der Befragten den Faktor Schneesicherheit an (bis maximal 3\%). Dennoch würden durchschnittlich 30\% der Befragten bei schlechten Schneeverhältnissen in eine höher gelegene Destination ausweichen oder gänzlich auf den Wintersport verzichten, was die Auswertung der offenen 
Frage nach möglichen Alternativen im Fall von schlechten Schneeverhältnissen ergeben hat. Dabei reagierten die Gäste von Davos am flexibelsten. 34\% der Gäste in der Wintervorsaison und 44\% in der Winterhauptsaison würden nicht mehr anreisen. In Scuol hingegen reagierten die Gäste am gelassensten: „nur“ 8\% der Befragten während der Wintervorsaison und 29\% während der Winterhauptsaison würden die Destination bei schlechten Schneebedingungen nicht wählen. In allen drei Destinationen war der Anteil derjenigen, die bei schlechten Schneeverhältnissen nicht mehr anreisen würden, während der Befragung zur Winterhauptsaison am höchsten.

Tab. 3: Anteil der befragten Gäste bzw. Wintersportler, die entweder in der geschlossenen oder offenen Frage "Schneesicherheit" als wichtig angaben.

\begin{tabular}{lllll}
\hline & \multicolumn{2}{l}{$\begin{array}{l}\text { Angaben von „Schneesicherheit“" } \\
\text { in der offen gestellten Frage „Was } \\
\text { sind die wichtigsten zwei Gründe } \\
\text { für die Anreise?" }\end{array}$} & $\begin{array}{l}\text { Angabe von „,wichtig“ in der } \\
\text { geschlossenen Frage „Wie wich- } \\
\text { tig ist Ihnen die Schneesicher- } \\
\text { heit?" }\end{array}$ \\
\hline Braunwald & Total Gäste & Wintersportler & Total Gäste & Wintersportler \\
Davos & $0.3 \%$ & $0.5 \%$ & $68.5 \%$ & $82.1 \%$ \\
Scuol & $6.3 \%$ & $10.3 \%$ & $80.8 \%$ & $90.3 \%$ \\
\hline
\end{tabular}

\section{Diskussion}

Aus verschiedenen Studien (vgl. Pröbstl 2006, Studer u. Christoffel 1990) ist bekannt, dass künstlich beschneite Pisten in sonst schneefreier Umgebung unter Wintertouristen sehr unbeliebt sind. Auf der anderen Seite wird die künstliche Beschneiung - zumindest von der Seite der Tourismusverantwortlichen - als wesentliche Anpassungsstrategie an Klimaerwärmung, kürzer werdende Wintersportsaisons und steigenden wirtschaftlichen Wettbewerbsdruck angesehen. Die künstliche Beschneiung dient nicht bloß der kurzfristigen unternehmerischen Risikoabsicherung, sondern erlaubt die Verbesserung der Pistenqualität und stützt das Image einer Destination im nationalen und internationalen Wettbewerb. Die beschneibare Pistenfläche in der Schweiz steigt stetig und hat aktuell den Stand von einem Drittel der gesamten Pistenfläche erreicht.

In der Tat zeigen die Resultate der Befragung auch unter den Gästen eine zunehmende Akzeptanz der künstlichen Beschneiung im Vergleich mit älteren Studien (vgl. Studer u. Christoffel 1990; Bürki 2000). Dennoch offenbaren sich diesbezüglich in der aktuellen Studie deutliche regionale und saisonale Differenzen. Dies deutet auf eine Abhängigkeit der Einstellung gegenüber Kunstschnee von der Gästestruktur, die sich je nach Ort und Saison ebenfalls unterscheidet (Aktivitäten, Lifestyle, Alter etc.). Die Ansprüche der Gäste an die touristische Infrastruktur (Bergbahnen, Pisten, Beschneiung) sind insbesondere in Davos und Scuol 
sehr hoch. Gerade im Winter reisen dort die meisten Gäste vorwiegend für den Wintersport an. Dementsprechend hoch ist auch die Akzeptanz der künstlichen Beschneiung. In Braunwald hingegen wird die wenig ausgebaute Infrastruktur (Pisten, Beschneiung) von vielen geschätzt. Die Ruhe und das Genießen der intakten Bergwelt sind ihnen lieber als Massentourismus, wie man ihn in Davos oder auch Scuol vorfindet. Der Wintersport ist für viele Gäste von untergeordneter Bedeutung und dementsprechend bleibt die Akzeptanz von Kunstschnee auch im Winter eher gering.

Kenntnisse über die Präferenzen und Verhaltensweisen der Gäste einer Destination müssen bei der Entwicklung von Strategien für die regionale touristische Angebotsplanung unbedingt beachtet werden. Ein Ausbau der Infrastruktur und des Angebots muss nicht unbedingt heißen, dass zusätzliche Gäste gewonnen werden können, sondern dass je nach dem ein bestimmtes Gästesegment auch „vergrault“ werden und der Destination dadurch verloren gehen könnte. Die Ergebnisse der Gästebefragung geben verschiedene Hinweise zur zukünftigen Entwicklung des Tourismus in den drei untersuchten Destinationen. In Davos sind das Skigebiet und ein breites Wintersportangebot sehr wichtige Attraktivitätsfaktoren. Zukünftig wird Davos aufgrund seiner Höhenlage bis $2844 \mathrm{~m}$ ü. M. trotz Klimaerwärmung weiter schneesicher bleiben, so dass eine skitouristische Inwertsetzung von Davos sinnvoll erscheint. Dazu könnte auch der maßvolle Ausbau der künstlichen Beschneiung gehören. Es ist allerdings unklar, wie sich das allgemeine Interesse am Wintersport entwickeln wird. Rebetez (2006) geht mittel- bis langfristig von einem nachlassenden breiten Interesse am Skisport aus. In Davos sind aber die Voraussetzungen gegeben (Infrastruktur, Gastronomie, Outdoorund Sportartikeleinzelhandel etc.), um Wintersporttrends wie z.B. Snowtubing (Reifen-Rutschen) oder Speed Flying (Kombination von Skiern und Gleitschirm) schnell aufzugreifen und spezielle Nischenmärkte zu besetzen. Wie in Davos sind auch in Scuol das Skigebiet und der Wintersport wesentliche Attraktivitätsfaktoren. Jedoch ist die Schneesicherheit in Scuol trotz der Höhenlage bis $2785 \mathrm{~m}$ ü. M. wegen der geringeren Niederschläge im Unterengadin kritischer zu sehen als in Davos. Ausgehend von dem allgemeinen Trend, dass die Gäste häufiger aber kürzere Ferien verbringen, steht Scuol zudem vor der Herausforderung, das touristische Angebot zu erweitern und auf regionalspezifisch einmalige, nicht beliebig ersetzbare Angebote zu setzen. Denn als Tagesausflugsziel kommt Scuol aufgrund der vergleichsweise langen Anreise nur für wenige in Frage. In Braunwald steht der Skitourismus an einem Scheideweg. Soll der Ort als Wintersportdestination weiter existieren, sind Investitionen in Aufstiegsanlagen und großflächige Beschneiungsanlagen notwendig. Da das Skigebiet zwischen 1300 und $1900 \mathrm{~m}$ ü. M. liegt und kaum ausbaufähig ist, ist die Schneesicherheit allerdings auch mit großflächiger künstlicher Beschneiung zukünftig kaum zu gewährleisten. Zudem würden diese Maßnahmen viele Gäste, darunter viele Stammgäste, verärgern.

Schneesicherheit ist eine wichtige Voraussetzung für den wirtschaftlichen Erfolg des Wintersporttourismus. Fehlt der Schnee witterungsbedingt oder als Folge 
der globalen Klimaerwärmung, gehen die Umsätze zurück. Schneesicherheit als Attraktivitätsfaktor ist zwar auch für die Gäste wichtig, allerdings nicht der einzige oder der entscheidende Faktor, der die Attraktivität einer Destination bestimmt oder das zentrale Motiv bei der Wahl der Destination darstellt. Schneesicherheit wird vermehrt als selbstverständlicher Teil des touristischen Angebots verstanden. Erst wenn der Schnee fehlt, beginnen auch die Gäste über die Bedeutung der Schneesicherheit für ihr Reiseverhalten nachzudenken. Insofern verhält es sich mit der Schneesicherheit ähnlich wie mit der Sicherheitslage eines Landes. Viele Menschen bereisen mit großer Selbstverständlichkeit die Länder dieser Welt. Erst kriegerische Konflikte oder übermäßige Kriminalitätsraten in einer Destination veranlassen die Reisenden, ihr Verhalten zu reflektieren.

Mit technischer Beschneiung können also nicht im Sinne eines „klassischen“ Attraktivitätsfaktors neue Gäste gewonnen werden, es kann aber das Ausbleiben der Gäste im Falle von schlechten Schneeverhältnissen verhindert werden. Zusammen mit Faktoren wie der Landschaft, des breiten Angebots touristischer Aktivitäten bildet die künstliche Beschneiung durchaus ein wichtiges Kriterium für den Erfolg des Tourismus in den Schweizer Alpen. 


\section{Literatur und Quellen}

Abegg, B. (1996): Klimaänderung und Tourismus - Klimafolgenforschung am Beispiel des Wintertourismus in den Schweizer Alpen. Vdf Hochschulverlag AG. Zürich.

Abegg, B., Agrawala, S., Crick, F. u. de Montfalcon, A. (2007): Climate change impacts and adaptation in winter tourism. In: Agrawala, S. (Hrsg.): Climate Change in the European Alps. OECD Publishing. Paris. S. 25-60.

Agrawala, S. (Hrsg.) (2007): Climate Change in the European Alps. OECD Publishing. Paris.

Bader, S. u. Bantle H. (2004): Das Schweizer Klima im Trend - Temperatur- und Niederschlagsentwicklung 1864-2001. Veröffentlichung der MeteoSchweiz, Nr. 68. Zürich.

Breiling, M., Charamza P. u. Skage O. R. (1997): Klimasensibilität österreichischer Bezirke mit besonderer Berücksichtigung des Wintertourismus. Endbericht des Forschungsauftrages des Österreichischen Bundesministeriums für Wirtschaftliche Angelegenheiten, Projektnummer 18 3895/222-I/9/95.

Bürki, R. (2000): Klimaänderung und Anpassungsprozesse im Wintertourismus. Ostschweizerische Geographische Gesellschaft. St. Gallen.

Elsasser, H. u. Bürki, R. (2002): Climate Change as a Threat to Tourism in the Alps. In: Climate Research, 20, S. 253-257.

Fauve, M., Rhyner, H. u. Schneebeli, M. (2002): Pistenpräparation und Pistenpflege - Das Handbuch für den Praktiker. Eidg. Schnee- und Lawinenforschungsinstitut SLF/WSL, Davos.

Frei, C., Calanca, P., Schär, C., Wanner, H., Schädler, B., Haeberli, W., Appenzeller, C., Neu, U., Thalmann E., Ritz, C. u. Hohmann, R. (2007): Grundlagen. In: Organe consultative sur les changements climatiques OcCC (Hrsg.), Klimaänderung und die Schweiz 2050. ProClim. Bern. S. 11-23.

Hermann, M., Heye, C. u. Leuthold, H. (2004): Grundlagen \& Techniken der empirischen Forschung. Geographisches Institut Universität Zürich.

International Commission for Protection of the Alps CIPRA (Hrsg.) (2006): Mit Schneekanonen gegen die Klimaerwärmung. In: CIPRA (Hrsg.), CIPRAINFO: Wintertourismus im Wandel, Nr. 81. Schaan. S. 7.

Job, H. (2005): Österreich - Die Alpen als Destination - eine Analyse in vier Dimensionen. Mitteilungen der Österreichischen Geographischen Gesellschaft, 147. Wien. S. 113-138.

Laternser, M. u. Schneebeli, M. (2003): Long-term snow climate trends of the Swiss Alps (1931-99). International Journal of Climatology, 23, S. 733-750. 
Légeret, B. (2006): Touristische Ausstattung und Abonnementspreise in Skigebieten. Diplomarbeit am Institut für Verkehrsplanung und Transportsysteme, ETH Zürich.

Mayer, M., Steiger, R. u. Tragwöger, L. (2007): Technischer Schnee rieselt vom touristischen Machbarkeitshimmel - Schneesicherheit und technische Beschneiung in westösterreichischen Skidestinationen vor dem Hintergrund klimatischer Wandlungsprozesse. Mitteilungen der Österreichischen Geographischen Gesellschaft, 149. Jg. (Jahresband). Wien. S. 157-180.

Meier, R. (1998): Sozioökonomische Aspekte von Klimaänderung und Naturkatastrophen in der Schweiz. Vdf Hochschulverlag AG, Zürich.

Müller, H., Weber, F. u. Thalmann, E. (2007): Tourismus. In: Organe consultative sur les changements climatiques OcCC (Hrsg.), Klimaänderung und die Schweiz 2050. ProClim. Bern. S. 79-94.

Neu, U. (2002): Temperaturentwicklung in der Schweiz im 20. Jahrhundert. In: Organe consultatif sur les changements climatiques OcCC (Hrsg.), Das Klima ändert - auch in der Schweiz. ProClim. Bern. S. 11.

Peck, S. (2005): Die Entwicklung der Wintersportinfrastruktur in Österreich von 1995 bis 2005. Diplomarbeit. Institut für Städtebau und Landschaftsarchitektur, Technische Universität Wien.

Pröbstl, U. (2006): Kunstschnee und Umwelt - Entwicklung und Auswirkungen der technischen Beschneiung. Haupt Verlag. Bern, Stuttgart, Wien.

Rebetez, M. (2006): Helvetien im Treibhaus - der weltweite Klimawandel und seine Auswirkungen auf die Schweiz. Haupt Verlag. Bern, Stuttgart, Wien.

Scott, D. u. Dawson, J. (2007): Climate Change vulnerability of the US Northeast (USA) Ski Industry. In: Matzarakis, A., de Freitas, C.R. u. Scott, D. (Hrsg.): Developments in Tourism Climatology. Freiburg. S. 191-198.

Scott, D. u. Jones, B. (2005): Climate Change and Banff National Park - Implications for Tourism and Recreation. Report für die Stadt Banff. University of Waterloo.

Scott, D. u. McBoyle G. u. Mills, B. (2003): Climate Change and the Skiing Industry in Southern Ontario (Canada) - Exploring the Importance of Snowmaking as a Technical Adaptation. In: Climate Research, 23, S. 171-181.

Seilbahnen Schweiz SBS (Hrsg.) (2008): Fakten und Zahlen 2008. SBS. Bern.

Steiger, R. (2006): Vulnerability and Adaptation of Winter Tourism in Tyrol and Bavaria. Am: Wengen 2006 Workshop - Adaptation to the Impacts of Climate Change in the European Alps, 4.-6. Oktober, Organisation for Economic Co-operation and Development OECD.

Steiger, R. (2007): Snowmaking - A Suitable Adaptation Strategy? In: Matzarakis, A., de Freitas, C. R.; Scott, D. (Hrsg.), Developments in Tourism Climatology, Freiburg, S. 178-182. 
Studer, N. u. Christoffel, J. (1990): Beschneiungsanlagen und künstlich erzeugter Schnee im Urteil von Skifahrern und Kur- und Verkehrsdirektoren. Diplomarbeit, Fakultät für Recht und Wirtschaft, Universität Bern.

Teich, M., Lardelli, C., Bebi, P., Gallati, D., Kytzia, S., Pohl, M., Pütz, M. u. Rixen, C. (2007): Klimawandel und Wintertourismus - Ökonomische und ökologische Auswirkungen von technischer Beschneiung. Eidg. Forschungsanstalt für Wald, Schnee und Landschaft WSL. Birmensdorf.

Trenberth, K. E. u. Jones, P. D. (2007): Observations - Surface and Atmospheric Climate Change. In: International Panel on Climate Change IPCC (Hrsg.): IPCC Fourth Assessment Report: Climate Change 2007, S. 235-336.

Witmer, U. (1984): Eine Methode zur flächendeckenden Kartierung von Schneehöhen unter Berücksichtigung von reliefbedingten Einflüssen. Geographica Bernensia G21. Bern. 


\section{Verzeichnis der Autorinnen und Autoren}

Dipl.-Geol. Manuela Armenat

Kontakt: Georg-August-Universität Göttingen, DFG-Graduiertenkolleg ,Interdisziplinäre Umweltgeschichte“, Bürgerstr. 50, 37073 Göttingen, E-Mail: marmena1@gwdg.de;

Axel Bader, M.A.

Kontakt: Georg-August-Universität Göttingen, DFG-Graduiertenkolleg ,Interdisziplinäre Umweltgeschichte“, Burckhardt-Institut der Fakultät für Forstwissenschaften und Waldökologie, Abteilung Forst- und Naturschutzpolitik und Forstgeschichte, Büsgenweg 3, 37077 Göttingen,

E-Mail: abader@gwdg.de;

Jessica Preutenborbeck, M.Sc.

Kontakt: Georg-August-Universität Göttingen, DFG-Graduiertenkolleg „Interdisziplinäre Umweltgeschichte“, Burckhardt-Institut der Fakultät für Forstwissenschaften und Waldökologie, Abteilung Naturschutz und Landschaftspflege, Büsgenweg 3, 37077 Göttingen,

E-Mail: jspical@gwdg.de;

Prof. Dr. Max Krott

Kontakt: Georg-August-Universität Göttingen, Burckhardt-Institut der Fakultät für Forstwissenschaften und Waldökologie, Abteilung Forst- und Naturschutzpolitik und Forstgeschichte, Büsgenweg 3, 37077 Göttingen,

E-Mail: mkrott@gwdg.de;

PD Dr. Achim Daschkeit

Kontakt: Umweltbundesamt (Dessau), Postfach 1406, 06813 Dessau-Roßlau, E-Mail: Achim.Daschkeit@uba.de;

PD Dr. Matthias Bürgi

Kontakt: Gruppe Landnutzungsgeschichte, Eidg. Forschungsanstalt WSL, Zürcherstrasse 111, 8903 Birmensdorf/Schweiz,

E-Mail: matthias.buergi@wsl.ch;

Esben Eidevik

Kontakt: E-Mail: Eidevik@yahoo.de; 
Dr. des. Urte Stobbe

Kontakt: Georg-August-Universität Göttingen, DFG-Graduiertenkolleg „Interdisziplinäre Umweltgeschichte“, Lange Geismarstr. 33/34, 37073 Göttingen, E-Mail: Urte.Stobbe@web.de;

Dipl.-Forstw. Christoph Riegert, Assessor des Forstdienstes

Kontakt: Georg-August-Universität Göttingen, Abteilung für Naturschutz und Landschaftspflege, AG Interdisziplinäre Forschung für Waldnaturschutz und naturverträgliche Waldnutzung, Büsgenweg 3, 37077 Göttingen, Tel.:

0551/393416, Fax: 0551/393415,

E-Mail: christoph.riegert@forst.uni-goettingen.de;

Dipl.-Biol. Ines Fleer

Kontakt: Bund für Umwelt und Naturschutz Deutschland e.V. (BUND), Landesverband Niedersachsen, Friends of the Earth Germany, Goebenstrasse 3a, 30161 Hannover, Tel: 0511/9656939,

E-Mail: ines.fleer@web.de;

Dipl.-Geogr. David Gallati

Kontakt: Kanton Thurgau, Staatskanzlei, Dienststelle für Statistik, Zürcherstr. 177, 8510 Frauenfeld/Schweiz,

E-Mail: david.gallati@tg.ch;

Dr. Tobias Reeh

Kontakt: Georg-August-Universität Göttingen, Geographisches Institut, Goldschmidtstraße 5, 37077 Göttingen, Tel.: 0551/398074, Fax: 0551/3912140,

E-Mail: treeh@gwdg.de;

Prof. Dr. Gerhard Ströhlein (i.R.)

Kontakt: Georg-August-Universität Göttingen, Geographisches Institut, Abteilung Humangeographie, Goldschmidtstraße 5, 37077 Göttingen, Tel.:

0551/398037, Fax: 0551/3912140,

E-Mail: gstroeh@gwdg.de; 
In dieser Reihe sind bisher erschienen:

Gee, K., Reeh, T. \& Kreisel, W. (Hrsg.) (2004): Regionale Identität, Tourismus und Landschaftsinterpretation - Eine natürliche Symbiose? (= ZELTForum - Göttinger Schriften zu Landschaftsinterpretation und Tourismus - Band 1). Göttingen.

Faust, H., Reeh, T. \& Gee, K. (Hrsg.) (2006): Freizeit und Tourismus - Konzeptionelle und regionale Studien aus kulturgeographischer Perspektive. (= ZELTForum - Göttinger Schriften zu Landschaftsinterpretation und Tourismus Band 2). Göttingen.

Reeh, T. \& Ströhlein, G. (Hrsg.) (2006): Zu Besuch in Deutschlands Mitte. Natur - Kultur - Tourismus. (= ZELTForum - Göttinger Schriften zu Landschaftsinterpretation und Tourismus - Band 3). Göttingen.

Reeh, T. \& Ströhlein, G. (Hrsg.) (2008): Natur erleben und Raum inszenieren. (= ZELTForum - Göttinger Schriften zu Landschaftsinterpretation und Tourismus - Band 4). Göttingen. 


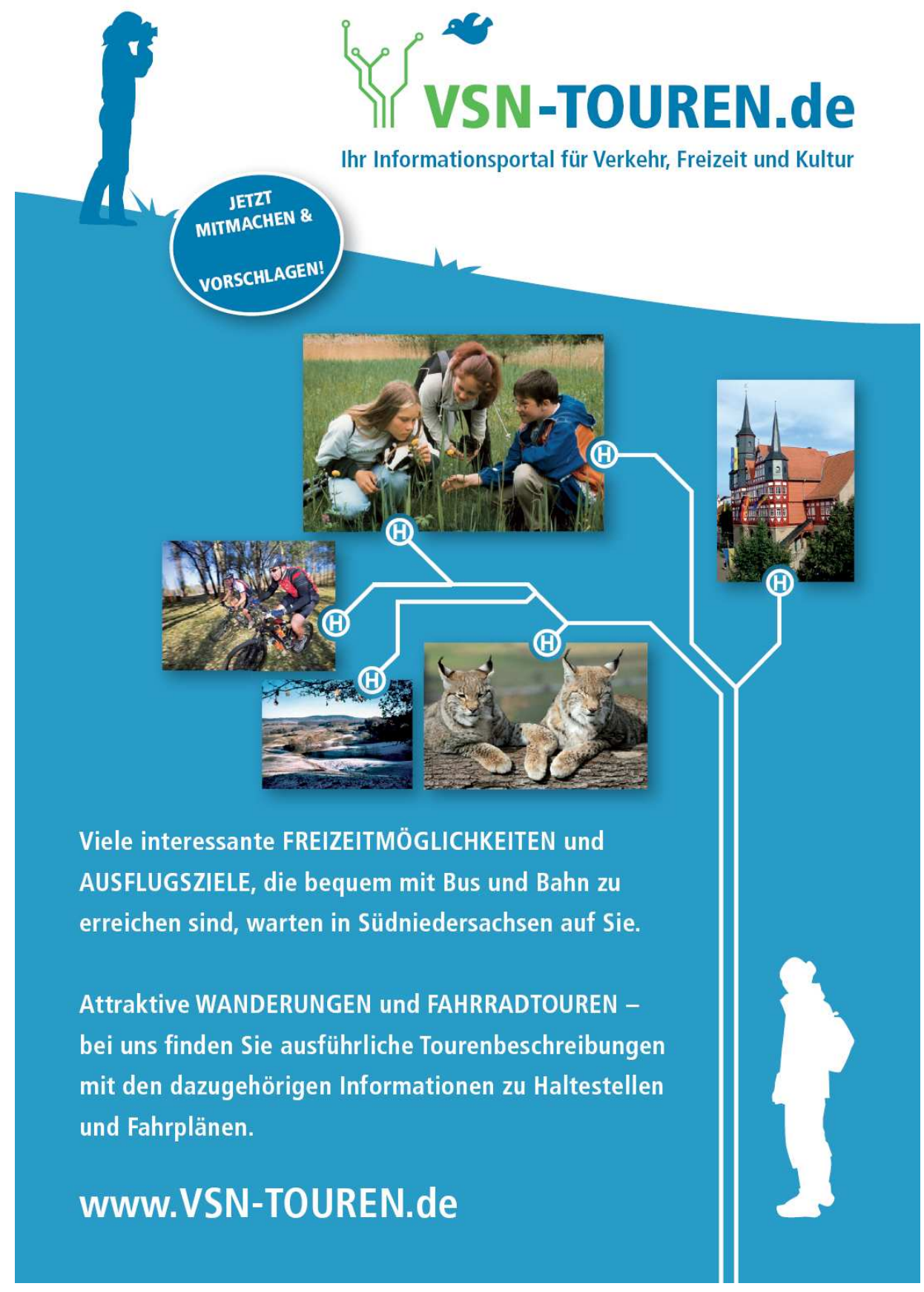


Für alle Regionen, die auf den Tourismus als ökonomisches Standbein setzen, ist ein marktfähiges Alleinstellungsmerkmal wichtig. Die Kulturlandschaft in ihrer jeweils besonderen Eigenart bietet hierfür ein endogenes Potenzial, das es zu erschließen, aber gleichermaßen auch zu schützen gilt. Doch viele kulturlandschaftliche Charakteristika und Besonderheiten eröffnen sich nicht unmittelbar, sondern müssen erst herausgearbeitet und „interpretiert" werden. Darin liegt die grundlegende Herausforderung. Der vorliegende Band beinhaltet zum einen theoretische Auseinandersetzungen mit dem Thema Kulturlandschaftsforschung aus der Perspektive verschiedener wissenschaftlicher Fachrichtungen, zum anderen praxisbezogene Fallbeispiele, die aufzeigen, wie die Forschungsergebnisse einem Freizeitpublikum auf interessante Art und Weise vermittelt werden können. Denn die Kulturlandschaft ist beides - wissenschaftliches Forschungsobjekt und Vermittlungsraum im Kontext touristischer Freizeitgestaltung.
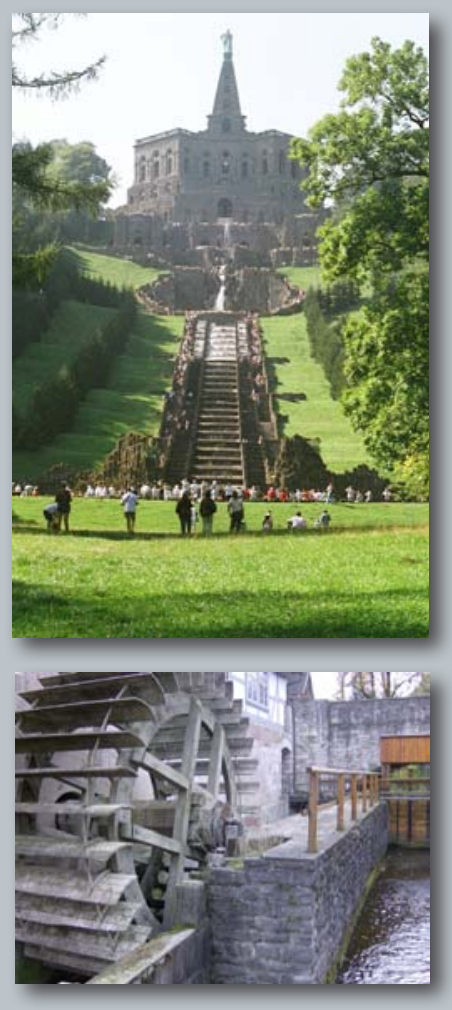

ISBN: $978-3-941875-33-3$

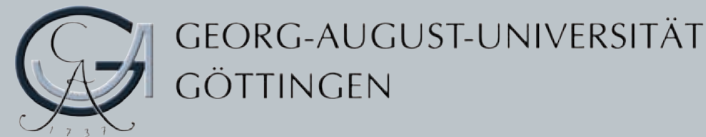

